\title{
Matériaux pour l'histoire de la sociologie de la connaissance
}

Giovanni Busino

\section{OpenEdition}

1 Journals

Édition électronique

URL : http://journals.openedition.org/ress/187

DOI : $10.4000 /$ ress. 187

ISSN : 1663-4446

Éditeur

Librairie Droz

Édition imprimée

Date de publication : 1 novembre 2007

Pagination : 57-190

ISBN : 978-2-600-01207-2

ISSN : 0048-8046

\section{Référence électronique}

Giovanni Busino, "Matériaux pour l'histoire de la sociologie de la connaissance », Revue européenne des sciences sociales [En ligne], XLV-139 | 2007, mis en ligne le 01 novembre 2010, consulté le 10 décembre 2020. URL : http://journals.openedition.org/ress/187 ; DOI : https://doi.org/10.4000/ress. 187

(c) Librairie Droz 


\title{
Giovanni BUSINO
}

\section{MATÉRIAUX POUR L'HISTOIRE DE LA SOCIOLOGIE DE LA CONNAISSANCE}

\author{
QU'EST-CE QUE LA SOCIOLOGIE \\ DE LA CONNAISSANCE SCIENTIFIQUE?
}

La sociologie de la connaissance occupe une place très importante dans le domaine des sciences de la société. Sa problématique principale a été, et est toujours, de rendre compte des propriétés sociales de la connaissance humaine.

Cette discipline a une longue histoire. Elle occupe une place considérable dans la tradition du savoir sociologique. Certains chercheurs en font remonter les origines à Francis Bacon (1561-1626), l'auteur du Novum Organum et d'une série d'autres travaux remarquables sur les sciences. D'autres affirment que les fondements de la discipline ont été posés par Karl Marx (1818-1883) puis par les pères fondateurs de la sociologie, notamment Vilfredo Pareto (1848-1923) et Emile Durkheim (1858-1917). Par la suite, cette branche de la sociologie a été illustrée en France par Maurice Halbwachs (1877-1945), Lucien Lévy-Bruhl (1857-1939) et Georges Gurvitch (1894-1965); en Allemagne par Max Scheler (1874-1928), Karl Mannheim (1893-1947) et tant d'autres; aux Etats-Unis d'Amérique surtout par Pitirim Sorokin (1889-1968) et Robert K. Merton (1910).

Des historiens des sciences sociales contestent cette thèse et affirment que la paternité de la sociologie de la connaissance revient au philosophe positiviste autrichien Wilhelm Jerusalem (1854-1923), auquel on doit la première tentative explicite, vers 1909, d'organiser en un véritable système conceptuel la discipline, qu'il avait nommée «Sociologie des Erkennens».

Quoiqu'il en soit de cette protohistoire et de l'histoire de la discipline, il faut noter que les chercheurs depuis la fin de la première guerre mondiale - contrairement à leurs prédécesseurs du XIX ${ }^{\mathrm{e}}$ siècle surtout préoccupés de la connaissance en général - ont réservé toute leur attention aux connaissances spécifiques, aux croyances particulières, aux savoirs ponctuels. Dès lors, ils nous ont laissé une grande variété de travaux aux résultats disparates, aux schémas interprétatifs diversifiés, contradictoires parfois et qui, pour toutes ces raisons, ne suscitent ni consensus ni accords parmi les sociologues. Voilà pourquoi il n'y a pas encore une définition acceptée unanimement de la sociologie de la connaissance et de ses liens avec les autres disciplines des sciences sociales, mais également avec la philosophie, avec l'épistémologie et avec la théorie de la connaissance.

Le plus célèbre parmi les sociologues contemporains spécialistes de la sociologie de la connaissance, l'américain Robert King Merton, a l'habitude de dire que le terme «connaissance» est si vague qu'il peut couvrir des contenus fort différents, voire opposés, par exemple la conscience, la compréhension, la 
représentation, l'intuition, le sentiment, les sensations, et contenir aussi une multiplicité de savoirs. En effet, le terme connaissance arrive à couvrir pratiquement tous les produits culturels: mythes, notions, concepts, images, modèles esthétiques, éthiques, scientifiques, etc.

Puisqu'il est pratiquement impossible de nous accrocher à une définition stable du terme de connaissance, Merton propose aux sociologues de prendre en charge avant tout les rapports existant entre la connaissance et les autres facteurs existentiels en vigueur à un moment donné, dans une société ou dans une culture déterminée. Cependant Merton n'ignore point que les rapports entre la connaissance et la société sont très complexes et compliqués. Pour cette raison, il nous invite à identifier et à différencier, dans le bloc de la connaissance, les savoirs propres aux savoirs quotidiens des savoirs savants, la connaissance ordinaire de la connaissance scientifique, les connaissances utilisées dans la vie de tous les jours, dans la vie quotidienne, de celles produites par des spécialistes, les connaissances du sens commun de celles qui sont systématisées par des pratiques disciplinaires rigoureuses.

Dans le séminaire de cette année, je me consacrerai exclusivement à ce dernier point, selon lequel la connaissance scientifique est un produit social, quoique des secteurs de cette même connaissance échappent, en partie ou entièrement, à la détermination de la société ou de la culture.

Cela dit, de quel type de connaissance spécifique s'agit-il? S'il y a différentes espèces et formes de connaissance, dont certaines sont plus fortement que d'autres connectées avec la structure sociale, l'analyse sociologique doit-elle en étudier la forme ou le contenu, la genèse ou l'acceptation, l'autonomie ou uniquement l'impact sur la vie sociale?

Mon propos est d'essayer d'établir si les efforts pour arriver à fonder une sociologie de la connaissance scientifique ont quelques probabilités d'aboutir à plus ou moins longue échéance. En d'autres termes, je me propose d'élucider la question suivante: si les produits de l'activité scientifique sont influencés par la société et par sa culture, s'ils subissent, comme tous les autres produits sociaux, des déterminations absolues ou relatives, comment peut-on affirmer que la science est universelle, objective, qu'elle échappe donc aux conditionnements sociaux et historiques? Mais si l'on admet l'autonomie de la science, qu'est-ce que le sociologue peut alors faire en ce domaine, qu'est-ce qu'il peut dire à son propos?

L'histoire de la sociologie nous aidera à comprendre comment les sociologues ont abordé cette problématique et de quelle façon ils ont envisagé de l'analyser. Mais avant de faire cet excursus historique, je dois vous rappeler que les sociologues ont souvent négligé, parmi toutes les connaissances spécialisées, les connaissances mathématiques en particulier, et les connaissances produites par les disciplines dites «dures » en général. Ils étaient persuadés que les sciences exactes et les sciences naturelles ne sont pas affectées par le social et par l'historicité, qu'elles ne sont pas conditionnées par les déterminants sociaux.

Que ces sciences dépendent dans une large mesure des procédés techniques dont l'homme dispose à une époque donnée et dans un cadre donné, que ces procédés techniques soient eux-mêmes subordonnés à l'évolution sociale leur paraissait peu important, car la distinction que ces mêmes sociologues établissaient entre science et organisation scientifique les débarrassaient du souci de 
prendre en charge l'histoire des techniques à une époque donnée et de résoudre ainsi des problèmes très complexes.

Certes, depuis environ six lustres, nous nous sommes occupés du travail quotidien des savants, des financements, des politiques publiques de la science, et nous avons écrits des livres et des articles sur les communautés scientifiques des savants et sur leur pouvoir de régulation de l'activité scientifique. Cependant, les travaux empiriques sur les modes de production de la connaissance dans les sciences «dures », sur la construction des théories scientifiques, sur leur diffusion et réception, sur leurs influences et emprises, ces travaux-là sont encore rarissimes, et ceci tout au moins jusqu'aux débuts des années ' 80 .

La raison de cette carence est notoire: le très fort impact sur le plan mondial de la sociologie de la science de Merton, une sociologie qui se place par ailleurs dans la droite ligne de la meilleure tradition sociologique, celle des pères fondateurs. Cette école de pensée, dominante pendant très longtemps dans la recherche sociologique, était fermement persuadée, sur la base peut-être d'un préjugé philosophique ou d'une option relevant de l'opinion commune, que la connaissance scientifique échappe à toute forme d'influence sociale, que les théories scientifiques possèdent un statut épistémologique spécial qui les soustrait à la compétence des sociologues. En vertu de ce statut, la connaissance scientifique se dérobe aux analyses des sociologues, car elle appartient aux philosophes et aux historiens. Dès lors, la sociologie de la connaissance scientifique couvre un domaine disciplinaire très différent de celui occupé par la théorie de la connaissance et par l'épistémologie des sciences. Elle ne s'occupe que des déterminants sociaux dans l'activité scientifique. Pour cette raison, elle se confond avec la sociologie de la science.

Cette position a été fortement contestée à partir des années ' 80 , grâce aux changements advenus, notamment pendant la décennie 1960-1970, dans la recherche philosophique et dans la recherche historique. Les travaux de T.S. Kuhn, de I. Lakatos, de P.K. Feyerabend, de S.F. Mason sur l'histoire des sciences de la nature, de R. M. Young sur le darwinisme et la biologie de l'évolution, et de tant d'autres... ont fourni les matériaux et les incitations indispensables au renouvellement des travaux récents de sociologie de la science et de sociologie de la connaissance scientifique.

C'est bien sous l'impulsion de la nouvelle philosophie de la science et sous l'impulsion de l'histoire des sciences et des techniques, qu'un certain nombre de sociologues ont proclamé que la science est un savoir pareil à tous les autres savoirs, que la sociologie de la connaissance doit étudier le fait scientifique comme n'importe quel fait social. D'autres sociologues ont commencé à faire une très nette distinction entre la sociologie de la connaissance, la sociologie de la science et la sociologie de la connaissance scientifique. D'après ces chercheurs, la sociologie de la connaissance étudie les déterminés, les déterminants et les formes de la détermination constituant la connaissance en général. La sociologie de la science s'occupe surtout de la science en tant qu'institution et profession; elle s'intéresse à l'organisation des activités scientifiques, aux influences sociales sur l'activité scientifique, sur son financement, sur son mode de fonctionnement; elle s'occupe du poids que la science exerce à son tour sur la société ainsi que des influences de la société sur la science. Par contre la sociologie de la connaissance scientifique vise à expliquer sociologiquement la construction des théories 
scientifiques, comment elles acquièrent une autonomie par rapport au contexte de production, deviennent universelles et pour quelle raison elles sont fort différentes de n'importe quel autre doctrine ou fait social. La sociologie de la connaissance scientifique étudie, par conséquent, les connaissances et les savoirs produits par des savants travaillant dans des institutions se vouant à la recherche et jouissant d'une apparente autonomie, d'une indépendance relative.

Cette tripartition est fortement contestée par des chercheurs anglais et français. Pour eux il n'y a qu'une seule approche raisonnable à l'étude sociologique de la connaissance scientifique, et cette approche doit unir la philosophie, l'histoire et la sociologie. Ces chercheurs utilisent les étiquettes «Social Studies of Sciences », «Science Studies», "Wissenschaftsforschung», pour indiquer leur domaine d'étude. Mais ces étiquettes ne satisfont pas tout le monde. Les Français hostiles à la tradition sociologique classique parlent couramment d'anthropologie des sciences et des techniques, tandis que les tenants de l'ethnométhodologie et du conflictualisme continuent à utiliser l'étiquette de sociologie de la science et ignorent celle de sociologie de la connaissance scientifique.

Pour ma part, je ne tiendrai aucun compte de ces distinctions. J'essayerai de montrer de quelle façon les sociologues ont étudié la science et les connaissances produites par elle, et de voir si l'approche sociologique de la science a une pertinence et une spécificité par rapport aux approches philosophiques et historiques.

\section{PHILOSOPHIE DE LA SCIENCE, HISTOIRE DES SCIENCES, SOCIOLOGIE DE LA SCIENCE}

Avant d'entrer dans le vif du sujet, il faut que je vous donne quelques informations préliminaires.

Depuis la nuit des temps, les hommes se sont interrogés sur le fait et sur la manière de connaître, sur les mécanismes qui nous permettent de construire ou de reconstruire, d'avoir présent à l'esprit un objet réel ou vrai, concret ou abstrait, physique ou mental. Depuis les Grecs, notamment, on s'est interrogé sur la faculté des hommes à élaborer des idées, à former des concepts ou à construire des images et des représentations. Puisque connaître signifie produire en pensée, reconstituer le mode de production des phénomènes, selon la belle formule de Jean Piaget (1896-1980); être conscient de l'existence de quelque chose consiste à saisir, à décrire, à expliquer les mécanismes de cette construction ou de cette reconstruction. Mieux, selon Jean Piaget, «Le point de départ de la connaissance est constitué par les actions du sujet sur le réel».

Les philosophes, notamment depuis Platon et Aristote, ont étudié les rapports entre le sujet qui connaît et l'objet à connaître. Ils ont donné un nom à cette activité cognitive: théorie de la connaissance, ou gnoséologie. Dans leurs innombrables écrits, ils établissent une distinction très importante entre une connaissance sensible ou empirique et une connaissance intellectuelle.

La connaissance empirique est le résultat de l'action immédiate des objets sur les organes du sens (vue, ouïe, toucher, odorat, goût); elle nous procure des sensations perceptives, immédiates, dont les impressions peuvent reparaître sous forme d'images mentales. En plus de cette connaissance empirique, il y a une connaissance intellectuelle, dite aussi conceptuelle, qui résulte de l'élaboration abstraite 
des données de la connaissance empirique et qui est constituée d'idées ou plus précisément de concepts; ces concepts ne représentent pas des réalités concrètes, mais des structures générales dont chacune caractérise une catégorie d'objets réels.

A l'intérieur de la connaissance intellectuelle, un territoire dénommé «Epistémologie» a été délimité. Les spécialistes de cette discipline, les épistémologues, n'étudient pas n'importe quel savoir, mais le savoir sur le savoir, la science, sa nature, ses méthodes, sa valeur, ses conceptualisations. J'ai dit la science et non les sciences, car les épistémologues s'intéressent à la science des sciences en général; c'est-à-dire aux principes fondamentaux qui se trouvent à la base des sciences particulières, au niveau de leurs classifications, c'est-à-dire de leurs rapports de coordination et de subordination. Ces classifications, vous vous en doutez, sont très nombreuses.

La plus célèbre est celle du physicien André Ampère (1775-1836), lequel classait les disciplines en sciences de l'esprit, ou sciences noologiques, et sciences cosmologiques, ou sciences des lois physiques de l'Univers. A sa suite, on parle encore de sciences culturelles et sciences naturelles, sciences de la compréhension et sciences de l'explication, sciences formelles ou sciences réelles, ou encore sciences idéographiques (système de signes susceptibles de suggérer les objets) et sciences nomothétiques (sciences recherchant les lois).

La dernière en date de ces classifications est celle de Jean Piaget. Le Maître distinguait les sciences nomothétiques, celles qui dégagent des lois, des sciences historiques dont l'objet est de reconstituer et de comprendre le déroulement de toutes les manifestations de la vie sociale au cours du temps.

Quoiqu'il en soit, toutes ces classifications n'entament pas l'unité fondamentale de la science, c'est-à-dire d'un savoir et d'une connaissance vrais, objectifs, valides, universels, produits grâce à des méthodes spécifiques.

Les visées des historiens sont tout autres. Ils sont confrontés à l'astronomie, à la biologie, à la mathématique, à la physique, etc., et jamais à la Science. Ils parlent de sir Isaac Newton (1642-1727), d'un savant qui est à l'origine de la physique moderne, mais aussi d'un chercheur épris de spéculations et d'exégèses bibliques, d'alchimie, d'un homme particulier, singulier, spécifique, n'ayant que peu de choses en commun avec ceux qui sont venus après lui ou qui ont vécu avant ou en même temps que lui. Pour l'historien, la trajectoire de Newton est unique, particulière, et dans cette perspective, il ne peut pas la réduire à la seule vérité ou à l'objectivité des lois de la gravitation universelle et de la décomposition de la lumière.

Edgar Ascher (Problèmes du relativisme, in «Revue européenne des sciences sociales », XXVII, 1989, n. 83, pp. 87-122) affirme que l'histoire des sciences est doublement relativiste, puisque elle doit reconstruire l'époque étudiée et le faire avec les idées et les moyens de l'époque à laquelle appartient l'historien. Ce dernier «ne peut pas traiter des documents comme de simples objets d'un cabinet de curiosités ou comme produits purement littéraires. Il doit tenir compte du fait que le scientifique du passé a voulu trouver des descriptions adéquates, des propriétés pertinentes et des lois vraies au sujet des phénomènes qu'il a choisi d'étudier. Il faut donc prendre ces prétentions au sérieux et examiner si le scientifique a réussi 'dans ce qu'il a voulu faire' et essayer de comprendre les raisons de son échec éventuel» (p. 99).

La sociologie, quant à elle, envisage la question d'un tout autre point de vue. Lorsque les sociologues font de la sociologie de la connaissance scientifique, ils 
visent surtout à mettre en évidence «les corrélations entre la connaissance et les autres facteurs existentiels de la société et de la civilisation», selon la définition classique de R.K. Merton. En d'autres termes, les sociologues essayent d'étudier les rapports existant entre l'activité cognitive et le contexte social, d'établir en somme des corrélations entre les connaissances des divers milieux et les particularités de ces mêmes milieux, du contexte socio-historique.

La sociologie de la connaissance n'a pas la visée universaliste de la philosophie de la science ni la visée relativiste de l'historiographie. Elle se situe entre les deux. Pour cette raison, jusqu'ici, nous avons échappé à maintes difficultés et à quelques paradoxes bien connus des philosophes et des historiens. Cependant, dès que nous essayons d'étudier sociologiquement la science ou les sciences, d'autres difficultés, d'autres paradoxes et contradictions nous barrent le chemin.

Comment peut-on étudier un savoir se voulant universel, la science, un savoir arraché aux temps et aux contingences, indifférents aux rapports sociaux dans lesquels les hommes sont intégrés? Peut-on affirmer que les faits scientifiques ne sont pas tributaires de l'action du milieu social? Peut-on nier que certains faits scientifiques ont une nature sociale, propre à une société particulière, façonnée par les membres d'une collectivité?

A ces questions, les sociologues donnent aujourd'hui deux sortes de réponses: l'une dite internaliste, le «programme classique ou modéré» et l'autre externaliste, le «programme fort».

Il suffit de savoir, pour le moment, que le programme classique est minimaliste: pour lui, il y a des énoncés qui sont universels (par ex., 5 x $5=25$; l'hypothèse du continu est indécidable; la vitesse est une quantité exprimée par le rapport d'une distance au temps mis à la parcourir...), et il y a des énoncés qui sont relationnels (par ex., les taux d'intérêts, la peine de mort, les rôles masculins et féminins, les croyances dans le Nirvana et dans le Karma, etc.).

Le «programme modéré» affirme que la sociologie de la science et la sociologie de la connaissance scientifique doivent s'occuper des énoncés relationnels exclusivement. Elles doivent s'interdire l'étude des énoncés universels. Elles doivent s'en tenir, en conséquence, à l'étude de l'influence des valeurs morales, de la structure sociale ou des institutions scientifiques sur la production des chercheurs. Certes, il y a une influence de la science sur la société mais aussi de la société sur la science, sur son rythme de développement, sur le choix des sujets à traiter, sur le contenu même des travaux scientifiques. Cependant cela ne met pas en danger l'autonomie de la science, ni son objectivité, son universalité, ni non plus le caractère désintéressé de l'activité du savant.

Par contre, le «programme fort» est maximaliste: il n'y a pas un savoir objectif, universel. L'universalité de la science est une illusion. Il en va de même pour les notions d'objectivité et de vérité. La science doit être traitée par la sociologie de la même façon que la magie et les autres formes de savoirs aboutissant à des connaissances. L'autonomie de la science est un leurre. Il s'agit d'une parade élaborée à une époque donnée pour des raisons politiques. Il en va de même de la distinction entre science et technique ainsi que du partage du domaine de recherche sur la science entre la philosophie, la sociologie et l'histoire.

Ces questions seront analysées plus tard. Par conséquent, je peux fermer la parenthèse et revenir à mes propos principaux : quand et comment s'est formé, à l'intérieur de la sociologie de la connaissance, le domaine spécifique dénommé 
«sociologie de la science»? Qui sont les sociologues qui ont le plus contribué à la «fabrication» de cette discipline? Quelles sont les problématiques les plus importantes mises en évidence par l'étude de la science et des activités des savants?

\section{LA SCIENCE SELON LES PÈRES FONDATEURS}

Presque tous les sociologues du XIX ${ }^{\mathrm{e}}$ siècle ont été de l'avis que la science en tant que système objectif de connaissances méthodiquement établies échappe à la juridiction de la sociologie. La science serait indifférente aux déterminations sociales, étrangère aux idéologies.

Karl Marx lui-même était de l'avis que la «vérité scientifique est toujours paradoxale au jugement de l'expérience journalière qui ne saisit que l'apparence trompeuse des choses ». Certes, les remarques de Marx sur la science sont éparpillées dans tous ses écrits. Nous ne disposons d'aucune présentation systématique et cohérente faite par l'auteur lui-même. Cela donne lieu à diverses reconstructions conceptuelles et à des interprétations disparates, voire contradictoires.

Sans prendre position dans ce débat aux implications philosophiques et politiques certaines, une chose semble sûre: Marx partage la conception scientifique dominante en son temps. Pour lui aussi la science est le savoir par excellence. Pour lui aussi la science arrive à s'affranchir du contexte historique et des rapports sociaux caractérisant une époque donnée. En conséquence, les rapports entre la connaissance et la science, entre la science et l'histoire, entre l'objectivité et la subjectivité, ne sont pas nécessairement affectés par les déterminismes socioéconomiques ni même par les facteurs existentiels. La science est objective et universelle, même si ses utilisations peuvent être partisanes, au profit de la classe dominante. La science peut devenir un instrument au service de finalités contingentes, particulières, et ainsi contribuer au maintien de l'exploitation opérée par le mode de production capitaliste. Néanmoins la connaissance qu'elle produit est une connaissance objective, fondée sur le fait que le monde réel a un caractère matériel unitaire, que ce monde réel est la nature.

La science, selon Marx, existe indépendamment de la conscience que nous avons d'elle et des représentations que nous pouvons en donner. Elle reste toujours la source unique de cette conscience et de ces représentations. La science, connaissance de la matière, de la nature, et de la conscience que nous en avons, instrument absolument indispensable pour agir de façon efficace, ne peut être que vraie, objective, universelle.

Marx accepte, en d'autres termes, la distinction entre la science et les utilisations de la science. La science a un seul référent, un seul déterminant, la nature, tandis que les utilisations de la science sont conditionnées par la société, par la classe dominante, et en dernier lieu par le mode de production. Seule l'histoire de la philosophie peut rendre compte de l'évolution des doctrines scientifiques; alors que les utilisations de la science, dans un mode de production économique donné, doivent être expliquées sociologiquement, par le matérialisme historique.

Dans cette perspective, la science est une connaissance universelle. Ses développements et ses évolutions sont dictés par une logique tenant compte exclusivement des lois de la matière. La nature a toujours le dernier mot à dire sur la validité de la connaissance scientifique. 
La science peut agir sur la nature dans la mesure où elle arrive à se conformer aux lois naturelles, à les respecter, à s'y soumettre même lorsqu'elle veut les maîtriser.

Une telle conception est très répandue dans la culture occidentale du XIX ${ }^{\mathrm{e}}$ siècle. Emile Durkheim, par exemple, la partage très largement. En effet, pour ce sociologue, la science permet de nous affranchir du monde psychologique, de la pure subjectivité. La méthode expérimentale traduit notre dépendance vis-à-vis du monde. C'est à la science de nous faire retrouver l'ordre du monde. Dans un texte de 1909, il écrit que le savant «se met au centre du monde et le fait converger vers lui (au lieu de croire qu'il y est): c'est alors l'émancipation complète: non seulement acquiescement réfléchi, mais aussi possibilité de changer le monde».

On sait que Durkheim a tenté d'élaborer une explication sociologique de la genèse des catégories essentielles de la pensée humaine et des formes fondamentales du raisonnement, et ceci pour fonder rigoureusement son holisme, sa conception que la société est première par rapport à toutes les autres composantes constitutives de la vie sociale.

Durkheim est convaincu que les catégories de temps, d'espace, de genre, de nombre, de cause, de force, et ainsi de suite, varient d'un groupe social à l'autre, et à l'intérieur d'un même groupe social d'une époque à l'autre. Elles dépendent de facteurs historiques, donc sociaux, même si «nous ne savons pas exactement quels ils sont, mais nous pouvons présumer qu'ils existent».

Dans une étude parue en 1985 et traduite en française en 1994, Steven Collins nous rappelle très précisément de quelle façon Durkheim, malgré une approche très sociologique de la question, est parvenu à faire des catégories des institutions transcendant les individus et à soutenir qu'elles correspondent aux propriétés universelles des choses.

Sans entrer dans les détails, bornons-nous ici à nous remémorer ce que Durkheim a écrit dans Les formes élémentaires de la vie religieuse, livre paru en 1912.

Le sociologue français, dès les premières pages de son livre, se pose la question suivante: «Mais si les catégories ne traduisent originellement que des états sociaux, ne s'ensuit-il pas qu'elles ne peuvent s'appliquer au reste de la nature qu'à titre de métaphores?... Ainsi, en tant qu'elles nous servent à penser le monde physique ou biologique, elles ne pourraient avoir que la valeur de symboles artificiels, pratiquement utiles peut-être, mais sans rapport avec la réalité ».

Le fait que ces catégories soient construites avec des éléments sociaux ne nous permet pas d'affirmer qu'elles soient sans fondement dans la nature des choses. En d'autres termes, pour échapper au relativisme inhérent à l'option attribuant aux catégories et au raisonnement une origine sociale, Durkheim doit déclarer: certes, les catégories sont dégagées par le monde social, mais nous les retrouvons également ailleurs. «La société les rend plus manifestes, mais elle n'en a pas le privilège». Des «notions qui ont été élaborées sur les modèles des choses sociales peuvent nous aider à penser des choses d'une autre nature. Du moins, si, quand elles sont ainsi détournées de leur signification première, ces notions jouent, en un sens, le rôle de symboles, c'est des symboles bien fondés. Si, par cela seul que ce sont des concepts construits, il y entre de l'artifice, c'est un artifice qui suit de près la nature et qui s'efforce de s'en rapprocher toujours davantage. De ce que les idées de temps, d'espace, de genre, de cause, de personnalité sont construites avec 
des éléments sociaux, il ne faut donc pas conclure qu'elles sont dénouées de toute valeur objective. Au contraire, leur origine sociale fait plutôt présumer qu'elles ne sont pas sans fondements dans la nature des choses ».

Durkheim affirme péremptoirement que la genèse des notions fondamentales de la pensée ou des catégories provient de la religion et, par la suite, de la Société. Mais étant donné l'unité du monde physique et social, ces notions finissent par conquérir une certaine universalité et objectivité dans la mesure où elles traduisent correctement les lois naturelles. Précisément pour cette raison, dans la conclusion de Les formes élémentaires de la vie religieuse, Durkheim écrit ceci: «Nous avons même vu que les notions essentielles de la logique scientifique sont d'origine religieuse. Sans doute, la science, pour les utiliser, les soumet à une élaboration nouvelle; elle les épure de toute sorte d'éléments adventices; d'une manière générale elle apporte, dans toutes ses démarches, un esprit critique qu'ignore la religion; elle s'entoure de précautions pour 'éviter la précipitation et la prévention', pour tenir à l'écart les passions, les préjugés et toutes les influences subjectives. Mais ces perfectionnements méthodologiques ne suffisent pas à la différencier de la religion. L'une et l'autre, sous ce rapport, poursuivent le même but; la pensée scientifique n'est qu'une forme plus parfaite de la pensée religieuse. Il semble donc naturel que la seconde s'efface progressivement devant la première, à mesure que celle-ci devient plus apte à s'acquitter de la tâche».

En d'autres termes, Durkheim affirme que la science prend la place de la religion à la suite de la croissance et de la complexification de la société ainsi que des différenciations produites par la division du travail. Ces phénomènes épurent l'activité cognitive, la libèrent des contraintes et des déterminations sociales et en font une valeur en soi et pour soi. La science est le produit de cet affranchissement, de cette libération progressive. Ses théories demeurent relativement indépendantes des influences sociales directes. Au fur et à mesure que le développement de la société devient important, les techniques d'observation des phénomènes se perfectionnent, les conceptualisations deviennent rigoureuses et formelles et de plus en plus conformes aux réalités observées. Dès lors, les résultats scientifiques sont acceptés par tous puisque conformes au monde réel et par conséquent vrais, objectifs, universels. Un jour, la science et la raison scientifique remplaceront la religion et les connaissances jusqu'ici acceptées par un acte de foi.

L'analyse sociologique de la science, dans la perspective durkheimienne, doit se limiter aux moments où les sciences se confondent encore avec les croyances religieuses, où elles ne sont pas encore affranchies des contraintes sociales. Ensuite, l'analyse sociologique prendra en compte la naissance des communautés scientifiques, l'institutionnalisation de la méthode scientifique et des techniques pour éliminer les erreurs, les préjugés et les déformations intellectuelles. Elle pourra rendre compte de l'organisation de l'activité scientifique, des rôles professionnels et de bien d'autres aspects du travail dans les laboratoires, mais elle ne pourra rien dire sur la nature de la véritable connaissance scientifique totalement indépendante du contexte social dans lequel pourtant elle s'est développée. Plus une science s'enracine dans le monde physique, se conforme et se plie à lui, plus elle devient autonome et indépendante. L'astronomie, la physique, la biologie, selon Durkheim, en sont des exemples éclatants.

Durkheim reste un homme de son temps, comme Marx et beaucoup d'autres savants du XIX ${ }^{\mathrm{e}}$ siècle. Pour le sociologue français, la science reste la valeur 
suprême de nos sociétés, elle seule nous apporte des connaissances vraies, non déchirées par les conflits sociaux. Elle constitue un savoir commun à tous, un savoir positif, donc libre de toute contrainte et de toutes déterminations. En bref, un savoir vrai, point entaché d'idéologie.

Je n'ai pas besoin de montrer les limites d'une telle conception, par ailleurs partagée par presque tous les sociologues jusqu'à une date très récente.

\section{SCHELER ET MANNHEIM}

Depuis l'époque de Marx jusqu'aux années '20, les sociologues ont traité abondamment de notre problématique, ils ont étudié les relations entre l'existence sociale et la connaissance ordinaire et scientifique, mais ils n'ont jamais élaboré une vision systématique des connaissances socialement conditionnées.

Je cite, pour mémoire, un des penseurs parmi les plus représentatifs de cette époque: Max Scheler (1874-1928). Ses études de sociologie de la connaissance, traduites en français par Sylvie Mesure, se trouvent désormais réunies dans le volume Problèmes de sociologie de la connaissance (Paris, PUF, 1993).

Scheler soutient que les valeurs ont une existence objective et constituent la matière grâce à laquelle le sujet est en mesure de préférer « le bon au mauvais et le meilleur au moins bon». Il affirme aussi, contre la conception kantienne, la primauté de la valeur sur le devoir. En effet, il déclare que nous sommes dans un cosmos de valeurs que nous ne devons pas produire mais seulement reconnaître et découvrir, par l'intuition, le sentiment, la visée émotionnelle, en d'autres termes par une activité extra-théorétique, par une «mathématique du cœur» capable de saisir en stricte logique les essences des choses.

Scheler établit une hiérarchie des valeurs: sensorielles, vitales (noble/ vulgaire), spirituelles (le génie), esthétiques (beau/laid), éthico-juridiques (juste/ injuste), religieuses (sacré/profane), spéculatives (vrai/faux). Selon cette conception, les faits mentaux sont des faits d'expérience phénoménologiques distincts des faits pragmatiques, naturels et scientifiques. Ces derniers sont le produit de la «libre contemplation», de la technique et de l'expérimentation. Ces deux derniers facteurs ont appris à la science «à limiter son intérêt pour chaque aspect de la nature à la dimension quantitativement mesurable du monde et aux lois de l'enchaînement spatio-temporel des phénomènes selon leurs ressemblances et leurs différences, autrement dit: à ce qui se peut saisir comme dépendant des phénomènes possibles du mouvement » (p. 145). Fille de l'union entre la philosophie et l'expérience du travail, la science fonctionne grâce au modèle de la causalité et à une certaine théorie dynamique de la matière, «tous les deux anthropomorphiques» et donc incapables de «s'ancrer ni dans des lieux ponctuels de l'espace ni dans des points déterminés du temps objectifs... Leurs points d'ancrage devraient être à la fois extérieurs à l'espace et au temps, puisque ces forces doivent pour leur part expliquer et rendre compréhensible avant tout la matière dont, dans la sphère du phénomène objectif, se mettent en place les déterminations spatio-temporelles de la matière et de ce qui survient, ainsi que les relations qui s'établissent sous ce rapport» (p. 220). En conséquence, «la science positive elle-même conduit à des problèmes indubitablement métaphysiques » (p. 221). 
La place réservée à la science dans une telle sociologie est subalterne. Le sociologue doit analyser les facteurs réels et les facteurs idéels de la science mais l'étude des types de connaissances produites est du ressort du philosophe.

Le premier sociologue qui a travaillé ce domaine d'étude de façon systématique et exhaustive est un Hongrois de culture allemande, Karl Mannheim, né en 1893 à Budapest, professeur à l'Université de Francfort, d'où il a été chassé par les nazis en 1933. Depuis cette date et jusqu'à sa mort en 1947, il enseigna la sociologie à la London School of Economics and Political Science.

Mannheim est considéré comme le père fondateur de la sociologie de la connaissance moderne. Ses travaux en la matière sont importants et nombreux. Ses idées et ses cadres conceptuels ont constamment évolué de l'époque de Strukturanalyse der Erkenntnistheorie, ouvrage paru en 1922, aux Essays on the Sociology of Knowledge, publiés en 1952 et suivis des Essays on the Sociology of Culture, également posthumes, parus en 1956.

Une reconstruction analytique de sa pensée montre que sa sociologie, élaborée à une époque agitée et cruelle, celle des totalitarismes, ne constitue pas un système fermé ou achevé. La sociologie de Mannheim est traversée par diverses influences: le relativisme socio-historique, la doctrine de la mise en perspective, le principe weberien de compréhension et de conciliation ainsi que la doctrine de la marginalité, qui permettrait, selon son auteur, d'obtenir un décentrement et fournirait les moyens d'échapper à l'aliénation inhérente au conformisme social.

Ici, je ne vous parlerai que des idées de Mannheim à propos de l'étude sociologique de la science. Pour ce sociologue, en cela disciple de Max Weber et fidèle tenant de la tradition académique allemande, il faut nettement distinguer les méthodes et les concepts des sciences de la nature des méthodes et des concepts des sciences sociales et historiques. Les phénomènes naturels et les rapports existant entre eux sont constants et invariables. Ils sont statistiques et intemporels. Nous les étudions comme étant extérieurs à nous, comme des objets indépendants de l'esprit, objectifs; nous le faisons de façon détachée, impartiale, à l'aide d'instruments, de mesures vérifiées et contrôlées, et en recourant à nos sens, à nos facultés sensorielles qui nous permettent d'éprouver les impressions produites par les objets matériels. L'étude du monde matériel ainsi faite produit des données empiriques immuables et universelles, constamment vérifiables et validées par différents chercheurs. Ces données sont par conséquent dotées de tous les critères de vérité.

Une théorie est vraie dans la mesure où elle n'est pas contraire, pas différente, pas dérogatoire, aux objets matériels, extérieurs à l'observateur, autonomes et indépendants par rapport à sa construction théorique. Dès lors pour Mannheim la connaissance produite par les sciences de la nature demeure permanente, uniforme, objective, universelle, vraie.

Ceci étant, Mannheim ajoute aussitôt que les sciences de la nature se développent plus ou moins linéairement car elles corrigent progressivement les erreurs et, par conséquent, s'approchent constamment de la vérité. Cette dernière est conçue comme un jugement valide et vrai pour autant que l'expérience le vérifie et n'entre pas en contradiction avec lui ou avec la théorie qui le formule et le construit.

De façon synthétique, on peut dire que pour Mannheim la connaissance scientifique évolue régulièrement en accumulant vérités externes, ou empiriques, et 
vérités internes, ou logiques de coordination, sur un monde physique intrinsèquement stable.

Par contre, la société et la culture ne sont pas extérieures à nous comme l'est la nature, d'où l'impossibilité de les observer avec détachement, de nous décentrer par rapport à elles, de regrouper nos observations en série, de soumettre à mesure ou à estimation les faits culturels et les faits sociaux.

Pour catégoriser des phénomènes culturels, pour en saisir la signification, il faut interpréter la pensée des individus, le sens que les agents donnent à leurs actions. La pensée n'est pas observable à la manière des objets matériels, des objets du monde réel. Chaque individu, chaque groupe social a des valeurs et des sens spécifiques. En plus, ils varient d'une époque historique à l'autre, d'un groupe social à l'autre, d'un segment de ce même groupe à un autre segment. Le sociologue a aussi des valeurs; il partage celles de son groupe d'appartenance. Il peut difficilement s'en décentrer, se détacher de son monde d'origine. Il peut, certes, avoir une compréhension sympathisante de ce qu'il étudie, mais il ne peut jamais le considérer comme étant extérieur à lui. Son système de référence, par rapport auquel il apprécie et analyse les comportements ou les actions des acteurs sociaux, reste toujours à la base de son travail.

Pour toutes ces raisons, Mannheim considère qu'aucun produit culturel n'est intemporel et immuable. Tous les phénomènes socioculturels sont variables et en perpétuelle mutation, d'où le dynamisme de leurs significations, d'où la difficulté de cumuler les recherches dans les sciences historiques et sociales.

Dans ces sciences, il faut tenir compte des spécificités, des particularités, des différences, des changements. A chaque époque d'une société, à chaque stade d'un système culturel, il faut recommencer le travail. Le travail antérieur peut nous servir à comprendre les sociétés du passé, rarement les nôtres, celles dans lesquelles nous vivons. Pour ces raisons les sciences physiques, selon Mannheim, ont une nature assez spécifiques, dont la sociologie ne peut aucunement faire l'économie. Pour lui, les facteurs existentiels, les conditionnements sociaux sont marginaux, subalternes dans ces sciences. La genèse historico-sociale d'une idée scientifique, les conditions qui ont rendu possibles son développement et sa vérification dans une organisation, dans un laboratoire, dans un organisme de recherche, n'ont aucun effet sur le contenu, sur la forme et la validité d'une théorie, sur sa vérité, sur son objectivité, sur son universalité. Les erreurs et les errements d'aujourd'hui seront corrigés demain. Ils seront aussitôt oubliés par les savants, même si les historiens continueront à les raconter dans leurs livres. La distinction faite entre les sciences de la nature et les sciences sociales et historiques permet à Mannheim d'affirmer que les premières échappent à l'analyse sociologique tandis que les secondes en constituent la matière principale.

Cette affirmation constitue-t-elle vraiment le noyau dur de la sociologie de Mannheim?

Il m'est difficile de répondre à cette question par un oui. Mannheim a beaucoup écrit et dans ses textes on peut trouver des passages où il insinue le doute qu'en physique, aussi, la stabilité des structures catégorielles n'est pas définitivement établie. A ce propos, les exemples fournis par les recherches de la physique quantique ou de celle des hautes énergies lui paraissent emblématiques. Dans cette physique-là, il n'est pas certain que la connaissance scientifique soit intemporelle et immuable. Il faut reconnaître cependant que sur ce thème, Mannheim a 
laissé des notations rapides, jamais vraiment développées par une argumentation solide. Si nous voulons rester fidèles aux textes fortement construits, nous devons alors admettre, voire reconnaître que le problème épistémologique central de la sociologie de Mannheim, comme par ailleurs de celle de Durkheim, est surtout celui de la relativité, ou comme les deux l'appellent: le relationnisme.

Selon cette épistémologie sociologique, toute pensée sociale, comme par ailleurs n'importe quelle forme de pensée extérieure aux sciences exactes, est relative à une position sociale donnée; elle est conditionnée par une perspective singulière, particulière, par l'intérêt de classe. Dans le domaine de la pensée sociale, il n'y a pas de critères universaux (des concepts et termes universels applicables à toutes les cultures et à tous les individus de toutes les sociétés) pour fonder la validité d'une assertion spécifique, caractéristique, sui generis. Et même la sociologie de la connaissance n'échappe guère à cette contrainte.

Il a été objecté à Mannheim que si cela était fondé, nous ne disposerions d'aucun moyen pour valider une thèse quelconque, y compris celle qui affirme que la connaissance sociale est déterminée ou conditionnée par la société.

La parade de Mannheim à cette objection est bien connue. Il l'a formulée à peu près ainsi: les assertions des sciences de la culture sont de nature et de forme très différentes de celles des sciences naturelles, toutefois elles peuvent aussi nous fournir des connaissances vraies. Et pour rendre plausible cette thèse, Mannheim doit réviser tacitement certaines de ses propositions méthodologiques sur les sciences de la nature. En effet, dans le livre Idéologie et Utopie, paru en 1919 mais accru en 1929, il écrit que la méthodologie des sciences physiques, expérimentales et formelles est un produit dérivé de la «Weltanschauung», d'une vue métaphysique du monde, sous-jacente à la conception que la bourgeoisie en ascension se fait de la vie. Dans cette conception du monde de la classe bourgeoise, conception pétrie par l'idéologie du cosmopolitisme démocratique, la place de la connaissance personnelle, qualitative, intuitive, est dévalorisée d'une manière constante. Le statut de connaissance authentique est réservé exclusivement aux formulations validables universellement. Ainsi, on arrive à éliminer les connaissances produites par les classes subalternes, on peut taxer d'opinions personnelles ou partisanes tout ce qui n'est pas produit par la culture dominante, celle de la classe hégémonique dans le mode de production capitaliste. C'est l'hégémonie des bourgeois, leur domination sociale et politique, qui fait prévaloir la prééminence, dans la vie intellectuelle, de la connaissance scientifique et l'épistémologie élaborée à partir de celle-ci. Et c'est ainsi que tous les savoirs et toutes les connaissances sont mesurés et évalués à l'aune d'une connaissance imposée par l'idéologie d'une classe. Cette connaissance ayant la prétention d'être reconnue par tous comme étant la seule et unique valable et vraie.

L'influence du marxisme sur Mannheim est évidente. Cependant ce sociologue en fait un usage original et hétérodoxe. En effet, il s'emploie à élaborer une épistémologie alternative, a-marxiste, capable de donner la dignité de savoir aux sciences sociales.

Comment le fait-il? En partant du présupposé qu'il y a un monde physique et un monde social. Les sciences de ces deux mondes sont garanties par deux épistémologies différentes. La prétention de liquider les sciences sociales au nom de l'épistémologie des sciences dures, n'est rien d'autre, selon Mannheim, qu'une mystification idéologique. Tout en reconnaissant aux sciences dures une validité 
certaine, Mannheim admet que celles-ci également, dans certaines circonstances, sont conditionnées par le point de vue adopté par l'observateur-chercheur. Pour cette raison la vieille épistémologie positiviste, celle de la nature des choses, est devenue inadéquate, inappropriée. Il faut réviser cette épistémologie ainsi que la vieille notion d'objectivité, selon laquelle la dite objectivité n'est que la qualité de ce qui existe indépendamment de l'esprit.

Mannheim ne conteste point le fait qu'on puisse arriver à des conclusions objectives à propos d'un phénomène donné, que ces conclusions puissent être vérifiées par d'autres au moyen de procédures et d'instruments préliminairement définis. Mais il est de l'avis que ces conclusions «objectives» restent incomplètes, vu qu'elles ont été produites suite à l'adoption d'un point de vue ou d'une perspective spécifique. Pour cette raison, les conclusions changeront lorsqu'un contexte social nouveau fera surgir de nouveaux points de vue et des nouvelles perspectives. Il y a objectivité lorsque les chercheurs partagent le même point de vue de départ, utilisent les mêmes instruments de mesure, effectuent les expérimentations avec les mêmes protocoles. Si les chercheurs adoptent des schémas de référence différents, alors l'objectivité ne pourra être obtenue que de façon indirecte.

Mannheim considère que l'objectivité reste indissociable de l'accord intellectuel, du consensus sur les points de vue, sur les instruments et sur les protocoles de recherche; et surtout, il est de l'avis que l'accord-consensus est possible à condition que les résultats produits par une certaine perspective puissent être transférés dans une autre perspective, ou être compatibles entre eux.

Pour Mannheim il y a des systèmes de référence divergents, surtout dans les périodes historiques de transition. Il arrive donc qu'un savant doive choisir entre des points de vue opposés ou des perspectives différentes. Comment fait-il un tel choix?

En donnant la préférence à la perspective qui lui paraît la plus large, la moins contestable logiquement, celle qui lui semble capable d'embrasser le plus de données; en somme la perspective la plus compréhensive et la plus féconde.

Par ce biais Mannheim croit liquider la vieille épistémologie positiviste, celle de la correspondance entre les propositions théoriques et la réalité du monde observé, l'épistémologie de la correspondance entre les énoncés déduits et la réalité extérieure, celle selon laquelle toute théorie déductive ou inductive doit se rapporter à un objet, aux données contrôlables par les sens.

Ainsi Mannheim, tout en reconnaissant aux sciences dures la capacité de produire des connaissances objectives, place ces dernières à l'intérieur d'un contexte socio-historique donné. Le changement du contexte modifie l'objectivité, car un nouveau point de vue l'a conditionnée ou la conditionnera.

Peut-on affirmer que Mannheim a abandonné sa doctrine, d'origine néokantienne, de la connaissance scientifique en tant qu'ensemble de vérités universelles et statiques? Peut-on dire aussi que la connaissance du monde physique et celle du monde social dépendent du type de questions que nous formulons à leur sujet, des finalités que les sujets cognitifs se fixent et des situations socio-historiques dans lesquelles ils agissent? Non. Mannheim se révolte à l'idée que la connaissance scientifique puisse être envisagée comme socialement conditionnelle, contingente, semblable à la connaissance socio-historique. Pour lui, les deux types de connaissance ne sont pas analogues. Il s'agit de connaissances distinctes, parallèles, disposant d'épistémologies différentes. Le relationnisme de 
la science physique reste pour lui un cas spécial du principe général du relationnisme. Mannheim suggère que la connaissance produite par les «social scientists» est en étroite connexion avec la position sociale qu'ils occupent et le contexte social dans lequel ils agissent. Par contre, la connaissance produite par les chercheurs en sciences naturelles subit un seul conditionnement, celui du temps et de l'espace.

D'un certain point de vue, Mannheim affirme que la connaissance physique et la connaissance sociale sont intrinsèquement limitées et susceptibles de révisions; et d'un autre point de vue que la nature de ces deux connaissances n'est pas la même et qu'elles subissent des déterminations, des conditionnements, des contraintes fort dissemblables.

En conclusion, Mannheim a dit et répété que l'épistémologie positiviste ne concerne que les sciences de la nature, qu'elle est inadaptée aux sciences sociohistoriques. Il a essayé d'élaborer une épistémologie appropriée aux sciences sociales, une épistémologie relationnelle, capable de rendre compte des déterminismes sociaux et existentiels agissant dans le monde. Selon lui la vieille épistémologie n'est plus susceptible de rendre compte des problématiques de la physique des quanta et des hautes énergies, mais elle ne peut pas encore être abandonnée. En attendant, la connaissance scientifique est différente pour lui de la connaissance sociale: la première dispose d'une logique indifférente aux conditionnements existentiels, tandis que la seconde est déterminée par la société et par sa culture, par la condition et la position de classe des acteurs sociaux.

Position étrange et contradictoire, en vérité, que celle de Mannheim. Malheureusement, les sociologues n'ont retenu qu'une seule thèse de son enseignement: la science est un domaine étranger à l'analyse sociologique.

\section{LA SOCIOLOGIE DU SAVOIR}

Ernst Grünwald a publié en 1934 un volume intitulé Das Problem des Soziologie des Wissens. Versuch einer kritische Darstellung der Wisssenssoziologischen Theorien (Wien-Leipzig, Braumueller, 1934; nouveau tirage, Hildesheim, Olms, 1967) par lequel il a voulu montrer que les difficultés de la sociologie de la connaissance et de la sociologie de la science dérivent de l'incertitude ou de l'ambiguiité de leurs postulats de base. Ces postulats peuvent essentiellement être ramenés, selon Grünwald, à deux visions du monde.

Les sociologues qui adhérent à la première, donnent pour acquis le fait que l'homme ne peut être compris en dehors du contexte historico-social dans lequel il vit. Ses pensées et ses idées proviennent du contexte historico-social qui les détermine, les conditionne, les façonne. A l'inverse, les sociologues qui adhérent à la seconde vision du monde demeurent convaincus que l'homme est partie intégrante de la nature, que la connaissance provient exclusivement de l'expérience empirique et que l'erreur est un éloignement de l'expérience empirique produit par des intérêts sociaux. Toutefois, l'erreur est toujours susceptible de correction, à condition bien évidemment que nous soyons capables de revenir à l'expérience et de nous soumettre à ses impératifs.

Le premier postulat est de nature historiciste: l'historicisme rejette les approches selon lesquelles les présuppositions et la structure de la connaissance 
sont fondées sur une théorie universelle de la nature humaine; l'historicisme insiste sur le fait que la connaissance est fondée localement et située historiquement. Suivant cette approche, le monde doit être considéré comme historiquement changeant.

La nature du deuxième postulat est psychologico-naturaliste. L'homme est partie intégrante du monde naturel. La connaissance ne peut se baser que sur l'expérience empirique de ce monde naturel. Le fait de ne pas se soumettre à l'expérience empirique est à l'origine de l'erreur. Il est possible que des intérêts sociaux nous poussent à négliger ou à mépriser l'expérience, cependant la rectification et la correction restent toujours possibles à condition de revenir à la source de toutes les vérités, à l'expérience acquise des faits naturels, du monde réel.

Ces deux façons de concevoir l'homme et la nature sont à l'origine de deux traditions: celle pour qui la science est relative à une société donnée et qu'elle constitue, de ce fait, un savoir parmi d'autres; et celle selon laquelle la science est un savoir spécifique, singulier, parce que constamment soumis à l'expérience immédiate (le premier contact de la pensée avec l'univers matériel), à l'expérience physique (qui consiste à agir sur les objets pour en découvrir les propriétés qui sont alors abstraites de ces objets comme tels), puis à l'expérience scientifique par laquelle tout est corrigé grâce à l'assimilation des choses à la raison, assimilation réalisée par abstraction à partir des coordinations entre les actions. Par conséquent, d'un côté nous aurions le savoir social soumis aux conditionnements existentiels, à la société, et de l'autre le savoir scientifique soumis aux conditionnements et aux déterminismes physiques, à la nature. Le premier savoir serait changeant, variable, local, particulier, tandis que le deuxième serait permanent, stable, global, universel. Certes, ce dernier savoir peut être dégradé par des conditionnements sociaux, mais il peut être rectifié, comme je l'ai déjà dit. Il peut donc être rétabli en son entièreté, alors que le premier est indéfectiblement inséparable du contexte social qui lui a donné la vie.

En d'autres termes, Grünwald veut démontrer que tous les sociologues qu'il étudie dans son livre doivent être rattachés à l'une ou l'autre de ces traditions, et que c'est bien leur type de rattachement qui détermine leur vision de la science en tant que savoir relatif parmi d'autres savoirs relatifs ou en tant que savoir singulier, spécifique, auto-organisateur, autonome, universel.

La position de Grünwald est, je crois, trop tranchée et intellectualiste. Peu de sociologues peuvent être rattachés sans autre à l'une ou à l'autre des deux traditions dont il est question dans son livre Le problème de la sociologie du savoir.

La séparation entre l'approche historiciste et l'approche psychologico-naturaliste n'est jamais aussi nette lorsqu'on est engagé dans la recherche de terrain. Marx, par exemple, n'est pas systématiquement le chercheur historiciste que Grünwald décrit. Durkheim et Pareto n'affichent pas systématiquement leur naturalisme ou leur psychologisme naturaliste. Tous ces sociologues, et Talcott Parsons l'a bien montré aux débuts des années '40, croient que l'histoire a un sens et une direction, qu'il y a des lois de l'organisation de la vie sociale, qu'il est possible de connaître ces lois en leur constance et uniformité, et que la science est le seul moyen à notre disposition pour les connaître ou les découvrir. Ces mêmes sociologues croient fermement que le contexte social peut voiler la véritable connaissance et que la tâche de la science consiste précisément à déchirer les voiles qui la dissimulent. 
Cette croyance n'est plus celle des sociologues d'aujourd'hui. Les sociologues d'aujourd'hui ne croient plus à l'existence de lois de l'histoire, à l'histoire comme progrès, au sens et aux fins de l'histoire, aux soleils de l'avenir, à une société sans conflits et sans aucune inégalité. Les sociologues contemporains veulent plutôt savoir pourquoi certains contextes sociaux limitent substantiellement la capacité des hommes à agir et pourquoi ces limitations changent dans le temps et dans l'espace. Ils veulent savoir aussi quelles sont les implications de ces mêmes limitations. Et ils se demandent également s'il y a un secteur de l'activité humaine, par exemple la science, qui échappe aux contraintes du contexte social. De ce point de vue-là, le livre de Grünwald n'est qu'un témoignage intéressant sur la culture sociologique de la fin des années ' 20 de ce siècle.

\section{LA SYSTÉMATISATION DE STARK}

Il est incontestable que la sociologie de la connaissance scientifique de Mannheim a joué un rôle important, en éloignant l'analyse sociologique de l'étude des sciences de la nature. On s'en rend compte en lisant les travaux de Werner Stark (né en 1909 et actif surtout à partir de 1947). Stark est l'auteur d'un ouvrage monumental The Sociology of Knowledge. An Essay in Aid of a Deeper Understanding of the History of Ideas, paru en 1958 (nouvelle éd., London, Routledge and Kegan Paul, 1967), et d'une Sociology of Religion en trois volumes, publiée à partir de 1966. En 1963, il fait paraître un essai The fundamental Forms of social Thought (New York, Fordham University Press) dans lequel on trouve une présentation systématisée de son approche de la sociologie de la connaissance.

Stark ne discute même pas le point concernant les rapports entre la science et l'analyse sociologique. Pour lui, il va de soi que la science est foncièrement étrangère aux analyses sociologiques. Stark ne fait que reprendre les thèses de Mannheim tout en s'employant à les étayer et à mieux les argumenter. Son but est de consolider pour de bon la thèse selon laquelle la science échappe à l'analyse sociologique ou, en d'autres termes, aux conditionnements existentiels.

Les conceptions de Stark sont exposées ex professo dans sa Sociologie de la connaissance. Ce livre est constitué de deux parties. Dans la première, il essaye de délimiter le champ de la sociologie de la connaissance tel qu'il s'est peu à peu formé, au cours du dernier siècle, grâce aux travaux des chercheurs; tandis que la deuxième partie énumère et discute les problèmes actuels étudiés et débattus par les spécialistes. L'ouvrage constitue un état de la question très précis, tel qu'on pouvait le dresser à la fin des années ' 50 .

Bien qu'il ne soit pas aisé de faire un résumé de ce gros livre, écrit en anglais mais pensé en allemand, je vais essayer tout de même d'en soutenir la gageure.

Dès les premières pages de son travail, Werner Stark insiste sur le fait que le monde physique se caractérise par sa permanence et par sa stabilité. Cela permet aux savants d'observer les phénomènes avec précision et de la manière la plus complète possible. Les physiciens arrivent à établir une correspondance constante entre la nature toujours invariante et leurs propres formulations théoriques. Dès lors, l'accord intellectuel entre les physiciens est relativement aisé. Cette conformité de pensée, ce consentement généralisé entre les membres de la même 
communauté de chercheurs, sont le produit de l'objectivité foncière de la connaissance scientifique.

Il n'en va pas de même dans toutes les sciences. Il y a une différence de taille et de nature entre les savants qui étudient le monde physique et ceux qui analysent le monde social. Les faits sociaux sont fabriqués par des individus socialement et historiquement situés, alors que les faits naturels nous sont donnés, ils sont là. Nous sommes inclus dans les faits sociaux, tandis que les faits naturels sont extérieurs à nous.

Après avoir soutenu qu'il y a une correspondance directe entre la véritable connaissance scientifique et les phénomènes naturels invariants, et que cette correspondance n'est jamais affectée par des facteurs socio-historique, Stark ajoute une deuxième thèse qu'il formule plus ou moins ainsi: la connaissance du monde physique est cumulative et fondée. Cette connaissance est élaborée dans le cadre d'une perspective visant à produire le maximum de résultats avec le minimum d'efforts, en vue d'obtenir une grande efficacité technique. En d'autres termes, Stark affirme que la science doit tenir les comptes des valeurs économiques et technologiques propres à une époque. Dès lors, la connaissance scientifique est liée à une perspective particulière, à une attitude spécifique, elle est animée par un souci de rendement, par l'efficacité. Ceci étant, elle ne peut donc être ni stable, ni permanente. D'une certaine manière, elle est aussi conditionnelle, contingente.

Stark écarte cette objection en ripostant que depuis l'aube de l'humanité, la valeur fondatrice de la science a toujours été la même: dévoiler les mystères du monde naturel, découvrir les lois de la nature, en maîtriser les processus, les soumettre à la volonté des hommes. Les savants n'ont pas d'autres alternatives devant eux. En conséquence, l'efficacité technique de la science reste un facteur marginal, secondaire, non nécessaire.

Le contenu factuel de la science est donc inaccessible à l'investigation scientifique, à l'enquête sociologique, car il ne s'agit jamais d'un cas d'espèce, d'un événement historique, mais toujours de données universelles. Le contenu factuel de la science est le produit de l'application cumulative de la même perspective à un monde naturel constitué ab aeterno.

Une telle conception de la connaissance scientifique a des implications très complexes. J'en mentionne quelques-unes.

La première, je la formulerais ainsi: si le corpus des connaissances scientifiques s'est constitué par accumulations successives au fil des siècles, il ne peut y avoir alors de changement de signification dans la base factuelle de la science. La véritable connaissance scientifique peut être formulée d'une seule et unique manière. Le corpus des connaissances s'accroît, certes, de plus en plus, d'année en année, mais sa base ne subit aucune mutation, aucune rectification, ni ajustement, ni calibrage.

La deuxième implication est celle-ci: les découvertes scientifiques sont très différentes des découvertes culturelles et artistiques, de celles que l'on peut observer dans le monde social. La découverte scientifique ne consiste jamais à produire de nouvelles significations, comme c'est toujours le cas dans les arts ou en philosophie. La science doit reconnaître des réalités existantes, en dévoiler les secrets. L'astronome, dit Stark, essaye de comprendre les données disponibles; ces données lui préexistent. Il doit s'y soumettre, se laisser conduire par elles. Le 
savant reste esclave de la vérité objective, de la réalité, tandis que l'homme de culture et le philosophe expriment toujours et surtout les valeurs qui leur sont chères. Bien entendu, Stark sait que les données scientifiques peuvent être conceptualisées et interprétées de façon différente et à des niveaux de généralisation disparate. Il sait aussi que certains niveaux de généralisation et certaines conceptions ne sont connectés que très indirectement aux observations empiriques. Mais à vrai dire, il en fait peu de cas. Il s'agirait de l'appendice métaphysique de la science, d'une extrémité inoffensive, anodine, aisément dissociable de la science véritable.

Comment départager la science véritable de son appendice métaphysique?

La réponse de Stark est assez simple et claire: la science s'interroge sur ce que sont les choses et comment elles fonctionnent, alors que la métaphysique est toujours en quête du pourquoi, des fondements. La science ne se demande pas, par exemple, pourquoi il y a une accélération constante de la chute des corps. Elle ne pose pas la question du pourquoi de la théorie de l'évolution des espèces. Elle se borne simplement à constater que la formulation théorique concorde avec les observations.

Je pourrais objecter, à ce propos, que la théorie de Darwin a été inspirée par l'analyse de la dynamique sociale de Malthus, donc qu'elle a été fortement influencée par des facteurs sociaux, mais Stark jugerait assurément cette objection peu pertinente, donc irrecevable. Pour lui, la théorie de Darwin est absolument vraie, elle synthétise un nombre important d'observations empiriques. Elle n'est ni révisable, ni corrigible. Elle n'est d'aucune manière influencée, disons de façon significative, par un facteur social quelconque.

Stark écrit à ce propos qu'il s'agit d'un point de doctrine universellement acceptée par tous les sociologues de la connaissance scientifique. Et il cite Marx, Lukacs, Mannheim, Alfred Weber et Merton qui auraient - à quelques nuances près - une même vision de la science. C'est possible, car ils étaient tous profondément imprégnés de la même philosophie standard de la connaissance scientifique. Une telle philosophie, exclusive et dominante pendant très longtemps dans les milieux académiques et intellectuels, n'envisageait qu'une seule position possible en matière de connaissance scientifique et d'activité intellectuelle. Et cette position reconnaissait, sans autre forme de procès, que la science se développait dans un monde où les problèmes existentiels n'avaient aucune place.

Je dois aussitôt reconnaître que la façon dont la sociologie de la connaissance scientifique a été élaborée, jusqu'à une époque récente, est tributaire de cette conception standard de la science, de cette philosophie qui postule que le monde est réel, objectif, préexistant, inflexible aux préférences et aux intentions de ses observateurs. Cette philosophie suppose que les caractéristiques de ce monde peuvent être tout à fait fidèlement représentées. La science est alors l'activité intellectuelle par laquelle nous décrivons exactement les objets, les processus et les relations qui font partie du monde des phénomènes naturels. Une bonne connaissance scientifique valide dévoile et révèle ces phénomènes et les incorpore à ses schémas théoriques.

Certes, dans le monde naturel il y a des mutations et des mouvements uniformes, variés, divergents, convergents, rayonnants; cependant ce même monde naturel est caractérisé par des uniformités immuables, des régularités, des constantes. Bien entendu, nous pouvons nommer «lois permanentes et 
universelles » ces uniformités, ces régularités empiriques, et cela pour une raison bien simple: elles nous disent ce qui arrivera en tout lieu, en toute circonstance, en tout temps. L'observation objective fournit les matériaux grâce auxquels les lois naturelles peuvent être correctement formulées. L'observation objective est assurée par les procédures expérimentales, par les contrôles, par une série de méthodes et de pratiques qui empêchent les déformations, les erreurs, les méprises des sens, et même les illusions produites par les préjugés ou les aveuglements engendrés par nos vanités et nos susceptibilités.

Que le corpus de la connaissance scientifique soit foncièrement empirique est un fait indiscutable. Cependant, ce même corpus contient aussi des généralisations dépourvues de lois d'observation, de lois ne pouvant pas être vérifiées. Ces propositions abstraites, spéculatives, jouent un rôle important dans le système de pensée scientifique. Ces propositions aident à comprendre des régularités observées et encore dépourvues de lois; elles coordonnent des lois d'observation se trouvant dans des systèmes de référence séparés, et parfois elles aident à révéler des phénomènes observables et jusque-là inconnus. Il arrive que ces propositions abstraites, ces spéculations, deviennent des lois d'observation grâce au développement des techniques expérimentales. Mais en attendant, ces propositions spéculatives n'ont pas le même statut que les lois d'observation, elles ne représentent pas le monde naturel de façon aussi rigoureuse que ce qu'exige la science. Il arrive que ces propositions, utiles pendant quelque temps, doivent être abandonnées parce qu'elles sont fragiles, contradictoires, parce qu'elles sont devenues inutiles. Le diablotin de James Clerk Maxwell (1831-1879), provisoirement utile à la théorie électromagnétique de la lumière; l'éther, dont on considérait qu'il remplissait le vide et servait de support à la propagation des ondes lumineuses; le phlogistique considéré lui aussi, pendant longtemps, comme un des matériaux de la composition des corps, sont des exemples probants de cet état de fait. Par contre, le contenu des lois d'observation n'est déterminé que par le seul monde physique.

Stark, comme par ailleurs presque tous les sociologues de la connaissance scientifique avant lui, ne distingue pas les lois d'observation des lois théoriques.

Les lois théoriques peuvent être révisées, abandonnées, corrigées, tandis que les lois d'observation jamais. Lorsqu'une loi théorique génère des inférences non confirmées par l'observation, elle est revisitée, révisée, ajustée aux faits, ou encore délaissée au profit d'une hypothèse alternative, ou de différentes hypothèses parmi lesquelles on choisira la plus solide, celle qui semble la plus plausible. L'hypothèse ainsi retenue devient alors une loi théorique. Les faits que l'ancienne loi théorique expliquait sont alors soumis à la nouvelle loi théorique et, grâce à celle-ci, ils sont mieux reliés les uns aux autres et ils offrent un schéma conceptuel plus performant.

Pour conclure sur ce point, je dirais que Stark a raison et tort en même temps: il a raison de soutenir qu'il y a des lois universelles, il a tort de ne pas distinguer les lois d'observation des lois théoriques. Il a raison de dire que les lois des sciences de la nature ne sont pas influencées par la société; il a tort de ne pas ajouter que cela est vrai pour les lois d'observation et l'est beaucoup moins pour les lois théoriques. Ces dernières peuvent dériver d'une idéologie, d'une métaphysique dominante, d'une croyance en vogue. Le contenu des spéculations théoriques peut varier, car il n'est pas déterminé par les données de l'observation et peut donc être conditionné par des intérêts sociaux. 
Il n'en reste pas moins que la connaissance des théories présuppose celle des faits; et celle des faits présuppose celle des théories. En d'autres termes, toute connaissance repose sur des règles, qui sont elles-mêmes normatives. Je veux dire par là que toute connaissance n'est pas réductible à du savoir positif. La science positive - la physique par exemple - est indépendante du temps et du lieu. Elle a affaire à des phénomènes reproductibles et prévisibles: la même expérience peut être répétée, à tout moment, dans n'importe quel laboratoire. De tels critères ne sont pas applicables à n'importe quelle espèce de connaissance: ni celle du passé - l'histoire - ni celle de la signification d'un texte - la critique littéraire - par exemple, ne peuvent être assimilées à des sciences positives. Il y a des sciences qui ne peuvent pas formuler d'énoncés prédictifs ni être testées en laboratoire. Mais cela ne veut pas dire qu'elles ne constituent pas une forme de connaissance. Si l'on admet l'existence de plusieurs formes de connaissance, alors les thèses de Mannheim reprises par Stark sont pertinentes. Si nous affirmons que la connaissance est une, alors ces thèses sont fausses et le problème du conditionnement social et existentiel de la science reste sans solution.

\section{LES THÉORICIENS DES IDÉOLOGIES}

Je dois très rapidement et très sommairement faire état, ici, des contributions offertes par les spécialistes de la théorie des idéologies à l'approfondissement de la théorie de la connaissance sociologique de la science et des sciences.

On sait que les sociologues appellent idéologie l'ensemble des idées et des images présentes dans des écrits ou dans des discours, mais aussi l'ensemble des comportements communs à une masse d'individus, voire à des institutions. L'idéologie permet au sociologue de comprendre le mode d'agir des hommes, les justifications qu'ils en donnent et qui les légitiment, c'est-à-dire les actions sociales et les systèmes de valeurs qui les fondent.

Précisément pour cela, notamment depuis le début du siècle, les sociologues ont essayé de nous dire d'où viennent les idéologies et à quoi elles servent. Il est entendu que les réponses à ces questions varient en fonction des écoles sociologiques, bien que certaines d'entre elles suscitent davantage d'adhésions que d'autres. Le marxisme, par exemple, a plus fortement marqué l'étude des idéologies que d'autres courants de pensée.

Pour les sociologues marxistes, l'origine des idéologies se trouve dans l'illusion d'une fausse conscience, dans les intérêts matériels des classes sociales, dans un mode particulier de production économique et de rapports sociaux, le mode de production capitaliste. Pour d'autres sociologues, l'origine des idéologies se trouve dans les passions et les sentiments humains, ou encore dans la nécessité d'argumenter des choix arbitraires, des choix qui sont à la base de n'importe quel système de valeurs, à la base de toutes les cultures.

Pour les sociologues marxistes, les idéologies assurent la domination d'une classe sociale; elles servent à situer les groupes sociaux dans les luttes politiques et sociales. Certes, elles sont des productions imaginaires, qui ne peuvent nous donner une véritable connaissance de la réalité. Pour certains marxistes, dès lors, il y a conflit entre la connaissance scientifique et la connaissance idéologique, tandis que pour d'autres, une telle distinction est irréelle dans les sociétés 
capitalistes où tous les savoirs, toutes les formes de connaissance sont contaminés par les intérêts de classe, donc affectés idéologiquement.

Pour les sociologues non-marxistes, les idéologies intègrent les individus à un groupe social. Elles les aident à percevoir et à lire la réalité sociale, à désigner leurs amis et à identifier leurs ennemis, à intérioriser les valeurs à poursuivre et à lutter pour stabiliser ou encore pour transformer une organisation, un groupe social ou une société. Certains de ces sociologues, critiques par ailleurs du marxisme, réhabilitent le rôle des idéologies. Pour eux, elles sont des états de conscience, des représentations, des discours pour justifier ou contester des choix politiques. Elles fournissent les liens entre les représentations discursives, les argumentations et les passions humaines, les sentiments.

Louis Dumont, pour ne citer que lui, affirme que les idéologies édifient la réalité sociale. D'après ce sociologue comparatiste français, les idéologies constituent des formes de connaissance pratique de la société. Il n'y aurait pas de société sans une ou des idéologies. Pour lui, l'idéologie est l'ensemble social des représentations, des idées et des valeurs communes à une société. Par exemple, l'idéologie économique est à la base de l'individualisme, donc de l'idéologie moderne qui constitue l'ensemble des représentations communes de notre civilisation.

Si les idéologies dissimulent ou masquent les intérêts pratico-politiques, ou si elles sont liées aux passions et aux désirs, aux nécessités de l'action, de la vie et de l'agir en société, quel est leur rapport avec la science et avec le travail des scientifiques?

Pour Marx et Engels, la structure économique de la société conditionne toutes les pratiques sociales, toutes les pensées, tous les sentiments, toutes nos manières de penser et de sentir. Cependant, et je l'ai déjà dit en son temps, ce conditionnement n'entraîne pas une relation nécessaire, inéluctable. Conditionnement ne signifie pas déterminisme: principe selon lequel, une fois que les conditions d'existence d'un phénomène sont posées, celui-ci doit inéluctablement se produire.

J'ai déjà dit que selon Marx et Engels l'idéologie, contrairement à la connaissance scientifique, ne peut fournir qu'une connaissance fausse de la réalité, une connaissance imaginaire, une connaissance qui falsifie les vrais problèmes et les perspectives du monde réel. L'idéologie est un rapport vécu et imaginaire que les hommes établissent avec leurs conditions d'existence. A ce titre, elle est un savoir explicatif de la réalité historique et sociale, elle peut fournir des explications compréhensibles et simples des faits et des événements de notre monde, mais elle reste une forme de connaissance biaisée, de méconnaissance de la réalité sociale et de l'action historique, contrairement à la science qui est un savoir explicatif, critique, vérifié, testé à l'aide de procédures expérimentales constamment remises en cause.

Contrairement à l'idéologie, la science n'est pas une lecture directe du réel, ni même une image ou une photographie de ce même réel. La science est un savoir relatif et provisoire, alors que l'idéologie est un savoir à prétentions absolues et définitives.

L'exemple le plus significatif de cette séparation entre la science et l'idéologie nous est offert par Vilfredo Pareto (1848-1923). Pour ce sociologue, l'idéologie masque toujours un programme, dissimule des intérêts, nous mystifie en nous 
faisant croire qu'un jugement de valeur est un jugement de fait. La science, par contre, est connaissance des choses, des faits. L'idéologie se fonde sur des absolus invérifiables, la science sur des constatations toujours contrôlables. La science est problématique et provisoire. L'idéologie est un savoir indiscutable et figé. La science est hypothétique, conjoncturelle; elle ne fait que poser des problèmes dont elle recherche la solution. C'est une connaissance sans sujet connaissant. Pour cette raison, la science s'accroît sans que nous en ayons immédiatement conscience.

Pour Pareto, la nature de la science est très différente de la nature de l'idéologie. La première avance par approximations successives tandis que la seconde fait appel à l'absolu, à l'indémontrable. La science est démonstration, critique, dépassement; l'idéologie est certitude, conviction, cristallisation. Une théorie scientifique peut être transformée en idéologie. Dans ce cas, elle n'est plus tenue à l'objectivité, au devoir de conformité avec les faits; sa seule obligation devient celle de l'utilité sociale. La finalité de la science est la vérité, celle de l'idéologie l'utilité. La science postule que le monde réel existe indépendamment de la connaissance que nous en avons, que nous pouvons connaître ce monde réel grâce à des procédures d'enquête, que la connaissance est vraie pour autant qu'elle s'approche du monde réel ou qu'elle est isomorphe à la structure de la réalité. Pour ces raisons, la sociologie ne doit pas étudier la connaissance certifiée en tant que telle, mais plutôt les conditions sociales favorisant la poursuite d'une connaissance objective. Au cours de cette étude, le sociologue devra mettre en évidence les idéologies qui rendent difficile cette recherche de connaissance et les opérations effectuées pour contourner les obstacles.

Les idées de Pareto seront reprises par d'autres et contribueront plus tard à consolider la conception standard de la connaissance scientifique. Je pense au travail réalisé par Gérard De Gré (né en 1915, professeur d'anthropologie et de sociologie, dès 1968, à l'Université de Waterloo, dans l'Iowa) pour faire accepter l'approche sociologique de la connaissance dans les Universités américaines. La démarche de De Gré a été magistralement décrite par François Chazel dans son article intitulé L'institutionnalisation de la sociologie de la connaissance aux Etats-Unis : l'apport de Gérard De Gré (paru dans la «Revue française de sociologie», XXVIII, 1987, pp. 663-677).

Cette approche de l'étude empirique de la science met surtout en évidence les modèles normatifs les plus stables des savants et néglige la signification sociale de leurs engagements cognitifs, pourtant constamment changeants. Cette approche, n'est pas indifférente aux facteurs économiques, technologiques et militaires. Tout en réservant une bonne place aux normes et aux valeurs de l'homme de science, à la nature de l'ethos scientifique, elle revendique avec rigueur la séparation de la science et de l'idéologie, de la science de la société. D'après cette approche, l'analyse scientifique ne doit rien avoir en commun avec l'analyse sociale, et donc avec les idéologies.

Nous verrons par la suite les métamorphoses de cette thèse chez Merton. Mais avant d'étudier les travaux du père fondateur de la sociologie de la science contemporaine, nous allons voir si les contributions de Sorokin, le maître de Merton et de Parsons, amorcent un changement dans la tradition qui sépare nettement l'analyse scientifique de l'analyse socio-historique. 


\section{LA SCIENCE SELON SOROKIN}

Pitirim Sorokin est un sociologue américain d'origine russe, né en 1889 et mort en 1968. Professeur à l'Université de Harvard, il a été le maître de Parsons, de Merton et de tant d'autres sociologues, mais il est surtout l'auteur d'une œuvre sociologique immense qui couvre presque tous les domaines de la sociologie. Ses contributions à la théorie de la connaissance sont nombreuses et toutes d'un très grand intérêt. Merton lui a consacré une très belle étude en 1963. Celle-ci constitue à présent le chapitre 6 de The Sociology of Science, livre paru en 1973. L'étude la plus complète sur Sorokin reste cependant celle du Belge Jacques Maquet, Sociologie de la connaissance. Sa structure et ses rapports avec la philosophie de la connaissance. Etude critique des systèmes de Karl Mannheim et de Pitirim A. Sorokin. Préface de F.S.C. Northrop, Bruxelles, Editions de l'Institut de Sociologie, 1969, IIe éd. Le point de départ de Sorokin est une affirmation de nature métaphysique: à la base de toutes les configurations sociales, il y a une prémisse majeure, un mode particulier de concevoir la vie. Cette prémisse conditionne et façonne tout ce qu'il se fait dans une société; c'est elle qui donne des significations à la vie sociale. C'est la variable indépendante, la variable explicative.

Sorokin affirme que ces prémisses majeures sont au nombre de trois: le sensualisme (la réalité et la connaissance de cette réalité nous sont données par les sens); l'idéationnel (la réalité est suprasensorielle, elle est l'œuvre d'un Être éternel et immatériel); l'idéalisme (la raison et la logique nous permettent d'élaborer des connaissances).

Toutes les sociétés humaines sont basées sur l'un ou l'autre de ces trois principes, sur l'une ou l'autre de ces trois conceptions ontologiques, c'est-à-dire sur des intellections pures dont l'existence serait prouvée et assurée par la seule définition.

Ces principes socio-culturels ont une fonction d'intégration. Ils rendent possible l'assemblage des éléments d'une configuration dans l'espace social et dans le temps. Ce sont eux qui donnent une cohérence, logiquement plus ou moins rigoureuse, aux diverses composantes d'un système ou d'une organisation. Ce sont eux qui donnent sens et signification à chaque élément.

Les trois grands systèmes socio-culturels (sensualisme, idéationnel, idéalisme) ont une structure, un agencement spécifique des parties entre elles, et une dynamique propre.

La cause des transformations de ces systèmes se trouve toujours dans chacun d'eux, jamais à l'extérieur du système. Sorokin rejette toutes les théories externalistes (environnementalistes, mécanistes, behavioristes) et affirme que «Tout système qui, au long de son existence, est un ensemble en mouvement, qui travaille, qui agit et qui ne demeure jamais en repos, ne peut faire autrement que de se transformer, précisément parce qu'il exerce longtemps une activité, un travail, et aussi longtemps qu'il existe». Cependant il y a une limite à ces transformations. Ces trois supersystèmes culturels disposent d'un nombre limité d'orientations, de rythmes, de formes, de types, d'allures générales. Pour cette raison ils sont condamnés à répéter les formes et les types, les rythmes et les orientations du passé. À défaut de cela ils risquent la disparition.

Le caractère des fluctuations des dynamiques socioculturelles dérive de cette contrainte propre à chacun des trois grands systèmes. Les transformations éven- 
tuelles ne sont jamais linéaires; elles comportent des discontinuités, des détours, des déviations.

Sorokin revendique l'existence d'une loi des processus culturels selon laquelle tout système passe successivement par une phase idéationnelle (où la vrai réalité est considérée comme suprasensible et suprarationnelle), par une phase idéaliste (où la réalité nous est donnée par la logique et la Raison) et par une phase sensualiste (où la réalité n'est autre chose que celle de l'expérience sensible).

Je laisse de côté la question de savoir comment Sorokin mesure les systèmes culturels, car les procédés utilisés me semblent artificiels et inintéressants. Je me borne ici à noter que même en admettant que chaque système évolue selon ses lois propres, il n'est pas possible de négliger le fait qu'il a également affaire à des formes extérieures, à d'autres systèmes socioculturels. Sorokin est convaincu que chaque système porte en lui-même les germes de ses propres transformations et que leurs interactions ne font que renforcer ces transformations. Les influences externes seraient secondaires.

C'est ainsi que la science, tout en obéissant à la loi immanente du changement, se trouve secondairement en interaction avec l'Etat, avec l'organisation économique et sociale, etc. Chacun de ces systèmes, tout en se transformant selon sa propre loi immanente, vient renforcer les transformations de la science. Sorokin s'applique à montrer que le système science, tout en formant une unité réelle, un système distinct, reste lié en même temps, dans un même ensemble culturel et à une même époque, avec tous les autres et englobe aussi un certain nombre d'éléments adventices.

Depuis quatre ou cinq siècles, l'Occident est dominé par le sensisme, doctrine selon laquelle la perception de la réalité nous est donnée par les sens. D'où notre intérêt pour les besoins physiques, pour la maîtrise et la modification du monde extérieur, pour les vérités empiriques. Même le contenu des catégories fondamentales (espace, nombre, causalité) diffère dans les diverses cultures. La science, tout en ayant une autonomie relative, dépend du supersystème culturel sensiste. Elle est un monde à part, obéissant à ses lois propres, mais dans le cadre d'une prémisse majeure, d'une conception ontologique, le sensualisme.

Une sociologie de la connaissance scientifique n'aurait, selon Sorokin, qu'une portée limitée. Seule la sociologie des dynamiques socioculturelles apporte des connaissances valables. Etudier la connaissance scientifique aide à comprendre certains mécanismes grâce auxquels les dynamiques socioculturelles fonctionnent. Mais pour comprendre les dynamiques de la culture, les changements civilisationnels, il faut aller au-delà d'une telle étude sectorielle.

La position de Sorokin est très critiquable et pourtant c'est grâce à lui que la sociologie de la science est née aux Etats-Unis. Ce n'est pas le moindre paradoxe dans la vie de ce sociologue davantage intéressé à la philosophie de l'histoire qu'à la sociologie.

\section{GURVITCH ET LA SCIENCE}

Un certain nombre des idées de Sorokin ont été reprises, développées et modifiées par le sociologue franco-russe Georges Gurvitch (1894-1965), actif dans les années '40-'60, et auteur de très nombreux travaux, parmi lesquels je ne citerai que Les cadres sociaux de la connaissance (Paris, PUF, 1966). 
Les changements d'approches, comme la permanence de certaines idées par rapport aux années '30-'50 sont évidents dans les élaborations sociologiques de ce professeur.

Pour Gurvitch, la connaissance scientifique «tend vers le désintéressement..., l'ouvert, l'accumulation, l'organisation, l'équilibre et la jonction entre le conceptuel et l'empirique. Elle part de cadres opératoires essentiellement construits, justifiés par les résultats acquis, lesquels appellent souvent une vérification expérimentale ». Cependant, pour ce sociologue, ce n'est que dans les sociétés de capitalisme concurrentiel que ce type de connaissance occupe un rang prédominant. La prétention de la connaissance scientifique d'être totalement détachée des cadres sociaux, est injustifiée et irréaliste. Pour Gurvitch, la connaissance scientifique n'a qu'une indépendance relative, même dans les sciences exactes. Dans les sciences de la nature interviennent régulièrement des coefficients sociaux, d'autant plus forts que la connaissance scientifique est plus développée. Les sciences dures se fondent sur l'expérience et l'expérimentation, qui sont toujours essentiellement humaines et subissent l'emprise de l'humain. Par ce biais, elles font intervenir le coefficient social dans la science. Toute nouvelle hypothèse porte l'empreinte de la structure de la société dans laquelle elle a été élaborée. Gurvitch affirme que même là où il est moins apparent, ce caractère est cependant présent, par exemple dans la physique des quanta. «La microphysique actuelle fait apparaître, inconsciemment, les limites de l'énergie atomique que les structures sociales globales en lutte dans les sociétés contemporaines peuvent déclencher».

Le poids du social est d'autant plus fort que la science contemporaine a besoin de laboratoires, d'organismes de recherche et d'expérimentation, nationaux et internationaux. «A l'époque de l'énergie atomique et de l'électronique, des planifications de la recherche scientifiques s'imposent. Aussi longtemps que les planifications publiques n'atteindront pas l'envergure indispensable, elles seront concurrencées par des organisations privées de recherche scientifique, liées aux trusts industriels, nationaux et internationaux ». Enfin, toujours selon Gurvitch, «une liaison entre science et réalité sociale... s'établit du fait que les connaissances scientifiques exigent des moyens propres à la diffusion de leurs résultats. Enseignement, vulgarisation, éditions de poche, radio ou télévision, tout un problème sociologique se pose concernant les voies de diffusion de la connaissance scientifique et leur maniement».

Les changements d'approches, comme la permanence de certaines idées par rapport aux années '30-'50 sont évidents dans les élaborations sociologiques de ce professeur.

Pour Gurvitch, la connaissance scientifique «tend vers le désintéressement..., l'ouvert, l'accumulation, l'organisation, l'équilibre et la jonction entre le conceptuel et l'empirique. Elle part de cadres opératoires essentiellement construits, justifiés par les résultats acquis, lesquels appellent souvent une vérification expérimentale ». Cependant, pour ce sociologue, ce n'est que dans les sociétés de capitalisme concurrentiel que ce type de connaissance occupe un rang prédominant. La prétention de la connaissance scientifique d'être totalement détachée des cadres sociaux, est injustifiée et irréaliste. Pour Gurvitch, la connaissance scientifique n'a qu'une indépendance relative, même dans les sciences exactes. Dans les sciences de la nature interviennent régulièrement des coefficients sociaux, d'au- 
tant plus forts que la connaissance scientifique est plus développée. Les sciences dures se fondent sur l'expérience et l'expérimentation, qui sont toujours essentiellement humaines et subissent l'emprise de l'humain. Par ce biais, elles font intervenir le coefficient social dans la science. Toute nouvelle hypothèse porte l'empreinte de la structure de la société dans laquelle elle a été élaborée. Gurvitch affirme que même là où il est moins apparent, ce caractère est cependant présent, par exemple dans la physique des quanta. «La microphysique actuelle fait apparaître, inconsciemment, les limites de l'énergie atomique que les structures sociales globales en lutte dans les sociétés contemporaines peuvent déclencher».

Le poids du social est d'autant plus fort que la science contemporaine a besoin de laboratoires, d'organismes de recherche et d'expérimentation, nationaux et internationaux. «A l'époque de l'énergie atomique et de l'électronique, des planifications de la recherche scientifiques s'imposent. Aussi longtemps que les planifications publiques n'atteindront pas l'envergure indispensable, elles seront concurrencées par des organisations privées de recherche scientifique, liées aux trusts industriels, nationaux et internationaux». Enfin, toujours selon Gurvitch, «une liaison entre science et réalité sociale... s'établit du fait que les connaissances scientifiques exigent des moyens propres à la diffusion de leurs résultats. Enseignement, vulgarisation, éditions de poche, radio ou télévision, tout un problème sociologique se pose concernant les voies de diffusion de la connaissance scientifique et leur maniement » Gurvitch tout en considérant que la science n'échappe point aux contraintes sociales, estime toutefois qu'elle en est relativement peu affectée et qu'elle arrive à ne pas se faire contaminer par les idéologies. Et pourtant, le même Gurvitch affiche une grande inquiétude, il craint que la connaissance technique parvienne un jour à dominer complètement les autres genres de connaissances, et notamment la connaissance scientifique. Il redoute que l'évolution des techniques, si elle continuait à être plus rapide que la transformation nécessaire des cadres sociaux, ne finisse par les assujettir et nous conduire finalement à un autoritarisme technocratique.

Pour Gurvitch, vous l'avez compris, la connaissance scientifique doit être distinguée de la connaissance technique. Cette dernière n'est pas simplement la connaissance des moyens employés pour atteindre des buts idéaux, ni même une connaissance scientifique appliquée, caractérisée par son élaboration standard et par sa transmissibilité. La connaissance technique est une connaissance irréductible aux autres formes de connaissances, et notamment à la connaissance scientifique. La connaissance technique «... est pénétrée par le désir de dominer le monde, de le manier, de le manipuler, de le commander». J'ajoute que Gurvitch ne limite guère la connaissance technique à la seule connaissance de la manipulation de la matière, qu'il n'identifie point la connaissance technique avec la technologie. Pour lui, la connaissance technique a un domaine plus vaste que le maniement de la matière: «C'est la connaissance de toutes les manipulations efficaces, artificielles et subalternes, mais qui ont tendance à se rendre indépendantes et à se valoriser comme telles - manipulations précises, transmissibles et novatrices, dont le savoir est inspiré par le désir de dominer le monde de la nature, de l'humain et de la société, afin de produire, de détruire, de sauvegarder, d'organiser, de planifier, de communiquer et de diffuser ».

Au début du capitalisme, les connaissances techniques se développent dans les fabriques et rarement en fonction des découvertes scientifiques. Pendant le 
$\mathrm{XX}^{\mathrm{e}}$ siècle, il y a eu tout d'abord jonction entre les connaissances techniques et les connaissances scientifiques, au gré des experts et des ingénieurs, puis domination de la technique. Dès lors, la science joue un rôle subalterne par rapport à la technique. Dans cette perspective Gurvitch écrit: "Nous sommes parvenus à une époque où les techniques débordent les structures sociales, et surtout les types de sociétés où elles ont pris naissance».

Jusqu'ici, jamais la technique n'avait engendré les cadres sociaux, au contraire, les cadres sociaux avaient suscité les techniques nouvelles et les avaient guidées et dominées. Le technophobe Gurvitch affirme péremptoirement: "Aujourd'hui, en revanche, nous assistons à un décalage saisissant entre, d'une part, les structures sociales et leurs œuvres de civilisation non techniques, et, d'autres part, les techniques. Les connaissances techniques, déchaînées, échappent à toute domination et à tout contrôle. Les techniques à leur niveau supérieur, laissent entrevoir leur force destructrice et les technocrates - ces apprentis sorciers - s'avèrent impuissants à maîtriser les forces qu'ils déchaînent. Les techniques sont emportées comme par un torrent, et il s'agit entre elles et les sociétés d'une course de vitesse».

Gurvitch distingue diverses formes de connaissances: la connaissance perceptive du monde extérieur, la connaissance des Autruis, des Nous, des groupes, des classes, des sociétés, la connaissance technique, la connaissance politique, la connaissance philosophique et la connaissance symbolique. Entre toutes ces connaissances et les cadres sociaux il y a des corrélations fonctionnelles.

En science, ces corrélations fonctionnelles ne sont pas toujours directes; en technique, elles existent de moins en moins. La technique serait devenue indépendante des ensembles sociaux. En tout cas, pour Gurvitch, science et technique continuent à être deux domaines séparés.

Que dire de cette sociologie, mieux: de cette construction non théorique?

Pour établir des corrélations fonctionnelles plausibles et raisonnables entre des types d'ensembles sociaux et des formes de connaissances, il faudrait tenir compte des multiples conditions imposées à la détermination des corrélations. Mais pour pouvoir le faire, il ne faudrait négliger aucun détail, ce qui dans la pratique est impossible à réaliser. Il arrive alors trop souvent que la mise en place de la corrélation explicative et de sa justification s'effacent devant ce qui n'est qu'une double description parallèle de ces cadres sociaux, puis des systèmes cognitifs, dits correspondants. Derrière tout cela, il y a le danger de produire autant de sociologies que de formes de connaissances énumérées.

Il y a chez Gurvitch des intuitions intéressantes, des développements plus serrés que chez Sorokin, mais trop de promesses non tenues. Aucune de ses notations n'est indifférente, mais de quelle façon déterminer, puis homogénéiser, sans négliger aucun détail, tous les éléments en jeu dans la «causalité singulière»? Comment justifier et fonder la corrélation étant donné que celle-ci a toujours affaire à deux variables uniquement, alors que dans la causalité singulière nous avons affaire à une distribution à plusieurs variables non appariées? Pour échapper à cette impasse, il faut alors renoncer à une «vraie explication» et aboutir aux descriptions parallèles dont j'ai parlé tout à l'heure.

Si elle veut rester explicative, la sociologie de la connaissance scientifique (ou la sociologie de la science) ne peut pas tout embrasser, elle doit tenir compte des logiques de la démonstration et de l'argumentation. Et l'une et l'autre lui impo- 
sent des contraintes formelles. C'est de là, à mon sens, que provient la fragilité de la sociologie de Georges Gurvitch, sa contribution mineure à l'histoire de la discipline.

\section{LA SOCIOLOGIE DE LA SCIENCE DE MERTON}

Robert King Merton (1910-2003), professeur de sociologie à Columbia University, est un ancien assistant de Sorokin (avec lequel il a signé des articles). Dès 1935, il se consacre essentiellement à l'étude des problèmes sociologiques posés par la science et aux questions fondamentales de la sociologie de la connaissance scientifique. Ses nombreuses publications, l'originalité de ses approches, l'étendue de sa culture, son érudition historique, en font un sociologue parmi les plus importants de sa génération et le fondateur de la sociologie de la science contemporaine.

Sa thèse de doctorat, soutenue en 1935 et imprimée en 1938, porte le titre: Science, Technology in Seventeenth-Century England, in "Osiris Studies on the History and Philosophy of Science», Bruges, Saint Catherine Press, 1938, vol. IV, pp. 360-632. Elle a été réimprimée, avec une nouvelle introduction, en 1970 à New York chez l'éditeur Howard Fertig. La même année une édition «Paperback» en était publiée par Harper \& Row à l'intention des étudiants en sociologie et en histoire.

Dans l'introduction à l'édition de 1970, Merton avoue que la lecture de Max Weber lui a donné l'idée d'étudier les relations existant entre le puritanisme, le développement de l'empirisme, la science et la technique, mais aussi d'établir la contribution de certaines sectes protestantes à la légitimation de la science comme institution sociale émergente.

Le livre traite, en onze chapitres, de l'émergence de la science et de la technique en Angleterre et des conséquences, pour la société, des solutions proposées par ces nouvelles formes de connaissances. Selon Merton, dès le XVII ${ }^{\mathrm{e}}$ siècle, l'institutionnalisation de la science a été, avec la propagation des «idées claires », le vrai ressort de la croissance scientifique. C'est elle qui a permis, en effet, d'organiser la recherche selon une stratégie à long terme, ce qui s'est révélé par la suite d'une efficacité singulière, même redoutable.

Sur la base d'une documentation impressionnante et éloquente, Merton montre que les valeurs qui étaient à la base du puritanisme et du piétisme suscitaient un intérêt croissant pour la science, et que les protestants manifestaient une attention plus soutenue envers les sciences naturelles que les adeptes d'autres religions.

La science anglaise du XVII ${ }^{\mathrm{e}}$ siècle n'a donc pas été exclusivement déterminée par les besoins sociaux et économiques de l'Angleterre de l'époque qui va du règne d'Elisabeth $\mathrm{I}^{\mathrm{re}}$ (1558-1603) à celui d'Anne Stuart (1665-1714).

Dans ce livre, Merton prouve, par exemple, que les recherches de météorologie ont certes pu déboucher sur des applications utiles à la navigation, au commerce, aux transports, mais que cet intérêt pratique n'a jamais été préalablement explicité par les Anglais du XVII ${ }^{\mathrm{e}}$ siècle. Il en a été de même pour une grande partie des travaux d'anatomie, qui furent pourtant, et sans aucun doute, d'une grande importance pour la médecine et pour la chirurgie.

Le même Merton a recensé toutes les recherches effectuées en Angleterre de 1661 à 1687 par les membres de la Royal Society et a calculé que le degré 
d'influence socio-économique sur la sélection des problèmes scientifiques représente environ $40 \%$ pour la science pure et $60 \%$ pour la science rattachée aux besoins socio-économiques. Mais si on regarde de très près les recherches ayant débouché sur des applications pratiques, on constate qu'environ $70 \%$ de ces recherches n'en prévoyaient explicitement aucune à l'origine. Pourquoi ?

La réponse de Merton est la suivante: «Dès le début, les membres de la Royal Society se préoccupèrent d'apporter une justification de leurs activités devant la Cour et le public profane, et s'employèrent dès qu'ils le purent à montrer les résultats pratiques de leurs travaux. La plupart des problèmes, étudiés de prime abord en raison de leur importance utilitaire, furent repris plus tard d'un point de vue plus désintéressé ». Voilà pourquoi Merton écarte l'hypothèse du déterminisme de la science ainsi que de son idéalisation. Il considère que le sociologue doit se borner à étudier le rapport dialectique entre le système social et la science, tout en sachant que cette dernière est gouvernée par des normes et des valeurs dotées d'une spécificité propre. Pour se développer, la science doit être soutenue par les valeurs d'un groupe social. Les changements affectant le système de valeurs d'une société peuvent influer sur l'essor de la science. Néanmoins, étant une institution sociale, la science jouit d'une certaine autonomie dans l'interdépendance dialectique.

La position de Merton laisse toutefois beaucoup de questions sans réponse: tout d'abord que signifie l'affirmation selon laquelle il existe un rapport dialectique entre le système social et la science? Faut-il entendre que la science est un système social en soi et pour soi? Est-ce que cela signifie qu'elle est un soussystème du système social, hétérogène, doté d'autonomie relative? Ou faut-il comprendre qu'elle est une fonction - parmi d'autres - du système social?

Merton ne donne aucune véritable réponse à ces questions, du moins explicitement, mais ses écrits suggèrent une position néo-fonctionnaliste: la science compte parmi les éléments (institutions), fonctionnels, dysfonctionnels, a-fonctionnels, du système social global.

C'est peut-être pour cela que l'analyse mertonienne de l'ethos (valeurs ultimes et idéaux d'un savoir, l'ethos est caractérisé par des normes et des impératifs institutionnels apparemment très éloignés de ceux en vigueur dans d'autres institutions, très différents des modèles de comportement dominants dans le système social global) est à l'origine des études sur la communauté scientifique, sur ses valeurs, sur le système social de la science.

Abordons maintenant l'examen de l'ethos scientifique, voire de l'ensemble des traits culturels caractéristiques du groupe des savants, des valeurs ultimes et des idéaux à la base du savoir de ce même groupe.

Merton a toujours défendu la thèse selon laquelle la culture de la science est hétéronome par rapport au système social. Cette culture de la science se fonderait sur quatre impératifs institutionnels : l'universalisme, le communalisme, le désintéressement et le doute systématique.

En d'autres termes, le système social de la science est régi par ces quatre normes idéales. Le respect de ces normes de la part de tous ceux qui participent à l'entreprise scientifique garantirait la rationalité, la cumulativité et le caractère non conflictuel du savoir scientifique.

Selon Merton, ces impératifs sont également des prescriptions morales et techniques, c'est-à-dire des ordres expressément formulés, assortis de toutes les 
précisions utiles à leur application. Ces impératifs proviennent de principes méthodologiques fondamentaux, réputés justes et bons dans un sens déterminé.

Ce sens déterminé est le suivant, toujours selon Merton: la finalité suprême de la science en tant qu'institution est d'accroître la connaissance vérifiée et vérifiable. Les principes méthodologiques fonctionnels à la base de cette finalité (et toutes leurs implications) possèdent nécessairement une connotation moralement positive, tout au moins dans la mesure où l'on présume qu'il est moralement positif de s'engager le plus possible afin d'accroître et d'améliorer la connaissance vérifiée.

La première et la plus fondamentale des valeurs qui sont à la base de l'ethos de la science est l'universalisme. L'universalisme se réfère au caractère intersubjectif du savoir, du fait de l'observance de certaines méthodes rigoureuses d'observation et d'interprétation.

D'après Merton, l'universalisme trouve son expression immédiate dans le canon (norme, règle, type idéal) selon lequel toute vérité prétendant être telle, et quelle que soit sa source, doit être soumise à des critères impersonnels préétablis, et doit être conforme à l'observation et aux connaissances précédemment établies. Le refus ou l'acceptation d'un énoncé scientifique ne doit pas être dépendant de caractéristiques personnelles ou sociales. La race, la nationalité, le sexe, la religion, la classe ou d'autres caractéristiques de l'homme de science sont insignifiantes en elles-mêmes et pour elles-mêmes.

L'objectivité, l'expérimentation, les observations, la vérification avec des instruments, le contrôle de l'interprétation des données, etc., bannissent le particularisme.

Les nazis n'ont pas pu, par exemple, empêcher les physiciens allemands d'utiliser la théorie de la relativité en raison de la judéité d'Albert Einstein (18791955), ni les anglophobes de mettre en doute la loi de la gravitation universelle formulée par Isaac Newton (1642-1727), ni les germanophobes de la refuser parce que le principe de l'attraction universelle avait été pressenti par l'astronome Johannes Kepler (1571-1630).

Lorsqu'en 1914, quatre-vingt-treize savants allemands, parmi lesquels Max Planck (1858-1947), créateur de la théorie des quanta et prix Nobel de physique en 1918, publièrent le célèbre manifeste dénonçant l'incompétence, le chauvinisme et la malhonnêteté de leurs collègues anglais et français, ces savants ne s'exprimaient pas en tant que scientifiques. Ils défendaient, en citoyens obséquieux, la politique de leur pays et pas du tout le caractère international, impersonnel, virtuellement universel de la science.

L'universalisme, en effet, exige que la carrière scientifique soit ouverte à tous ceux qui ont les compétences requises, indépendamment de leur nationalité, race, sexe, religion ou leur affiliation partisane. Il en va de l'intérêt de la science, et l'intérêt de la science à l'ouverture et au pluralisme est aussi à la base de l'éthique de la recherche libre.

Dois-je vous avouer que cet ethos de la science me paraît peu convaincant et bien fragile? Et pourtant ceux qui prétendent qu'il s'agit d'une idéologie font preuve, à mon sens, de simplisme. Il suffit de lire attentivement Merton pour s'apercevoir qu'il manifeste une grande circonspection en la matière.

Si sa vision de l'ethos me paraît fragile, c'est plutôt parce que sa construction n'a pas été bien formée. Merton met sur le même plan des énoncés appartenant à 
des niveaux logiques différents: l'objectivisme gnoséologique, la neutralité de la science, la logique de l'enquête, l'égalité des chercheurs dans la recherche, la liberté d'accès aux carrières n'appartiennent pas aux mêmes classes logiques, ne s'enracinent point dans le même terreau. L'universalisme de Merton mélange des choses fort hétérogènes: (a) un principe déontologique (les savants doivent être recrutés et sélectionnés sur la base du mérite, en faisant abstraction du sexe, de la race et de l'origine sociale); (b) un principe méthodologique (la reproduction des procédures et par là le contrôle des résultats obtenus); (c) un principe gnoséologique (l'objectivité conçue en tant que correspondance entre la réalité et la construction conceptuelle qui représente cette réalité); (d) un principe métaphysique (la vérité que l'objectivité garantirait en toutes circonstances).

Si l'on considère de près ces principes, on s'aperçoit très vite qu'ils sont dissemblables et disparates. Le principe déontologique dépend de l'éthique, des coutumes et des mœurs, des normes acceptées par un groupe social à une époque donnée. À l'époque de Weber, les femmes, les juifs, les socialistes étaient écartés de la recherche scientifique malgré leurs mérites. Il en a été de même, mais pour d'autres raisons, entre 1945 et la fin de la guerre froide, à l'encontre des communistes en Occident et des non communistes dans les anciennes démocraties populaires.

Le principe méthodologique est une affaire d'intersubjectivité, c'est-à-dire de situation de communication entre divers sujets. En nous rencontrant intentionnellement, en privilégiant certaines compétences, en utilisant le même langage, nous créons une forme supérieure de communauté, laquelle partage ainsi une même manière d'appréhender, de concevoir et de communiquer la réalité.

Les principes gnoséologique (l'objectivité) et métaphysique (la vérité) sont trop variables et incertains, ils ont un sens et une signification si différents d'une époque à l'autre, d'une civilisation à l'autre, qu'il serait opportun de mieux les spécifier, ou de les caractériser de façon plus rigoureuse.

L'universalisme de la science est une hypothèse intéressante, qu'il vaut la peine de creuser. La définition de Merton est cependant insatisfaisante, car elle reste évasive quant à la nécessité de définir la science par la relation à la totalité dans laquelle elle s'insère. J'ajoute que lorsqu'on lit les travaux de Merton, en oubliant ses écrits proprement théoriques, on est bien obligé de constater que son universalisme est nuancé, plus raffiné qu'on ne l'imagine, comme d'ailleurs aussi son objectivisme. Dans ses travaux sur la science en Angleterre ou dans son très beau livre Sur les épaules des géants (On the Shoulders of Giants, New York, Free Press, 1965; IIe éd., Orlando, Fl., Harcourt Brace Jovanovich, 1985), Merton évite toujours le piège de la doctrine selon laquelle la perception sensorielle permet une connaissance directe de la réalité extérieure. En effet, il ne fait jamais dériver la vérité du modèle logico-déductif, ni ne présume que la vérité soit le produit inévitable de la démarche scientifique.

La vérité de Merton sociologue est un processus. Elle n'est pas aussi pure, absolue, que celle de Karl Popper, ni même proche ou analogue à celle de la tradition purement objectiviste. Elle n'est pas totalement inhérente, interne à la logique de la recherche et à l'usage qu'en font les pragmatistes (pour lesquels il doit exister une connexion indissoluble entre la connaissance rationnelle et l'application pratique ou la fin rationnelle), ou à l'usage qu'en font les instrumentalistes (pour lesquels l'activité de recherche doit nécessairement produire un certain effet pratico-politique). 
Certes, lorsque Merton est confronté à deux théories scientifiques inconciliables, étrangères à la perspective qui prétend que la science avance selon des processus cumulatifs, il n'hésite pas à parler de faits universels, de faits de nature, bref à recourir à des mythes fondateurs. Mais cela n'est pas grave, selon moi, parce que le sociologue de Columbia University n'oblitère jamais le fait que la science demeure un fait social total, une construction historique.

Prenons à titre d'exemple ses travaux sur la science et l'ordre social, sur la science et la structure sociale démocratique, sur la machine, l'ouvrier et le technicien, travaux que vous pouvez lire dans la quatrième partie du livre Social Theory and Social Structure. Theoretical and Empirical Investigations (Chicago, University of Chicago Press, 1973). Que constatons-nous d'emblée et sans la moindre difficulté dans ces écrits? Que Merton sait parfaitement déceler les conditionnements socioculturels à l'œuvre dans les diverses communautés scientifiques, qu'il décrit les comportements, les valeurs et les choix effectifs des travailleurs de la recherche avec précision et réalisme. J'admets toutefois qu'il néglige l'étude de l'influence des facteurs sociaux sur les orientations de la recherche, et qu'il ne nous explique pas pourquoi, par exemple, les recherches sur le cancer ou sur le paludisme sont défavorisées, alors même que celles sur les maladies génétiques sont très largement soutenues; pourquoi on encourage les recherches sur les technologies éducatives et non les recherches sur les processus d'assimilation et d'intégration des Musulmans dans les pays européens.

J'admets également que Merton ne traite jamais des critères de validité des propositions scientifiques. Est-ce à cause de son attachement aux faits universels ? Est-ce dû à sa conception de la nature humaine ou à sa croyance en une science fondamentale pure, universelle, qui n'est pas, en dernière instance, source de conflits et de divisions insurmontables?

Pour éclairer ces ambiguïtés ou élucider ces paradoxes, il faudrait faire une sociologie de la sociologie de Merton, ce qui n'est pas mon propos. Je dois me borner à dire ici que pour Merton, la science est un système social autonome.

Je vous ai déjà parlé de l'universalisme. À côté de ce principe fondateur, il y en a d'autres, ainsi que je l'ai déjà mentionné. Ces valeurs-principes sont le communalisme (Communality), le désintéressement, et le doute systématique avec leurs compléments: la rationalité, l'utilitarisme, l'individualisme et l'ameliorative progress, le progrès améliorant.

Le deuxième élément de l'ethos scientifique est donc le communalisme. La norme du communalisme se réfère au caractère collectif de l'entreprise scientifique. La science est le résultat d'un effort coopératif, elle est un bien public. Puisque les produits scientifiques sont le résultat d'une activité coopérative, de travaux entrepris et effectués par les collègues d'hier et d'aujourd'hui, ces produits appartiennent à tous. Les droits des producteurs individuels ne sont pas absolus. La loi de la relativité ou la loi de Pareto ne font pas partie du patrimoine personnel d'Einstein et de Pareto, ni non plus de leurs héritiers. L'ethos scientifique réduit au minimum les droits de propriété sur les découvertes scientifiques. Certes, le découvreur en tire des bénéfices indirects: la célébrité, le prestige, les honneurs, des doctorats h.c., le prix Nobel. Il peut déposer un brevet d'invention qui lui donne un droit exclusif d'exploitation pour un temps déterminé, ou vendre ce droit à une industrie. Cependant les droits assurés par le brevet déposé portent sur les applications pratiques, économiques, de la découverte, jamais sur les 
énoncés théoriques, sur le fait que j'ai le droit d'étudier, de critiquer, de généraliser ou de citer la loi de la relativité ou celle de la distribution de la richesse.

Etant donné l'importance institutionnelle attachée à la réputation et au prestige, à l'originalité et à la renommée, il est inévitable de voir apparaître des sentiments de jalousie, d'envie ou d'antipathie entre les savants. Vous comprenez maintenant pourquoi les questions de priorité sont des questions fondamentales; vous saisissez pourquoi il existe une lutte acharnée afin de publier avant tous les autres. Est-ce un tel ou un tel autre qui a fait telle découverte? Est-ce que ce sont les Français ou les Américains qui ont découvert les mécanismes de propagation du SIDA, qui ont mis au point les tests permettant de mettre en évidence la présence du virus?

Les réponses à ces questions ont une importance capitale. Il en va tout d'abord du prestige de deux savants, prestige qui procure ensuite des moyens financiers considérables aux institutions auxquelles ces deux savants sont rattachés, et en plus il en va de la gloire et de la prééminence de la Nation française ou des EtatsUnis d'Amérique.

Faut-il vous rappeler les disputes entre les Anglais et les Allemands à propos du calcul différentiel, de l'étude des variations infiniment petites des fonctions mathématiques? Pour les Anglais l'inventeur est Newton, pour les Allemands Gottfried Wilhelm Leibniz (1646-1716).

Puisque la science fait partie du patrimoine commun, le secret n'a pas cours dans la recherche. Les savants doivent publier leurs travaux de sorte que tout un chacun puisse les étudier et en vérifier les résultats. Publier devient une contrainte, une obligation absolue: Publish or Perish.

Le communalisme scientifique mertonien est-il vraiment la pratique la plus courante parmi les savants? Oui, si l'on admet la distinction entre la science fondamentale et la science appliquée, entre la science et la technologie, entre la recherche pure et la recherche appliquée. Mais si ces distinctions sont rejetées, le communalisme scientifique n'est qu'un pur idéal. La pratique réelle est faite alors d'antagonismes, de compétition, de concurrence sans limites, de coups très bas.

Je pourrais dire la même chose du troisième élément de l'ethos scientifique, le désintéressement. Ce dernier doit contrebalancer toutes les motivations extrascientifiques des chercheurs. Il empêche la fraude tout autant que l'émergence des pseudosciences. Merton nous rappelle qu'il ne faut pas confondre le désintéressement avec l'altruisme, l'action intéressée avec l'égoïsme. L'altruisme et l'égoïsme relèvent de l'analyse des motivations; le désintéressement, par contre, relève de l'analyse institutionnelle.

Une institution de recherche, qui est très souvent assurée d'un soutien public ou privé, et donc moins préoccupée qu'une entreprise par des questions de financement et de rentabilité à courte échéance, est portée à travailler pour l'éternité, pour la gloire, pour l'Ewigkeit, comme disent les Allemands. Son activité est désintéressée. Dès lors, il est dans l'intérêt du savant de se conformer à cet état d'esprit, sous peine de sanctions ou de conflits graves.

Pensez à ce qui s'est produit en 1968, lorsque les savants découvrirent la vraie nature du projet Camelot financé par les Forces Armées des Etats-Unis d'Amérique. Ceux d'entre eux qui avaient accepté de prêter leur concours à une politique de mise au pas des mouvements progressistes latino-américains, eurent un très mauvais moment à passer. 
Merton présume que le caractère public de la science, le fait qu'elle soit une activité transparente, en tout cas contrôlée ou contrôlable, contribue à développer une éthique de l'intégrité, à garantir une attitude d'extrême sévérité vis-àvis de la tricherie et du plagiat. Certes, la compétition entre les savants est rude, l'esprit d'école courant, les médisances pas exceptionnelles, la jalousie - ici sous la forme d'émulation effrénée, là sous la forme de susceptibilité ombrageuse, de mesquinerie et de rancune - est monnaie courante. Les manœuvres en vue de bloquer l'ascension d'un collègue, de l'empêcher d'obtenir un financement pour la réalisation d'un projet de recherche, sont des pratiques de tous les jours. Bien sûr, tout cela peut rendre la vie quotidienne désagréable, voire difficile, mais pas pour autant détruire un chercheur de talent. Non reconnu dans son pays, il le sera assurément ailleurs. La communauté scientifique est lente, mais elle ne se trompe jamais dans ses reconnaissances. Négligés ici, ou même déconsidérés, les travaux d'un savant, s'ils sont de qualité, trouveront toujours des lecteurs attentifs ailleurs. En fait, cela n'est vrai qu'en partie. Je peux être un physicien de génie, mais si je n'ai pas de laboratoire, si je n'ai pas une position qui me permette de publier dans les revues importantes de ma discipline, d'accéder aux sources des renseignements, mes chances de réussite sur le plan international demeureront très minces.

Le quatrième élément de l'ethos scientifique est le doute systématique, c'està-dire une disponibilité intellectuelle constante à la critique et à la révision des acquis scientifiques. Le scepticisme organisé distingue la science de la nonscience, il abolit la distinction entre le profane et le sacré, entre ce qui doit être respecté sans critique aucune et ce qui peut être soumis à une analyse critique, au doute systématique. Le scepticisme s'oppose à toute certitude, empêche d'imaginer que quelque chose puisse être valable dans l'absolu, valide à jamais.

Là aussi Merton exprime un idéal. La pratique est autre: un savant qui critiquerait sa discipline et douterait de ses méthodes serait vite marginalisé par ses collègues.

Pourquoi? Parce que les critiques pourraient causer des dommages graves à l'image de la discipline, mettre en danger les financements, rendre précaire la carrière des savants.

Les quatre impératifs institutionnels, je l'ai déjà dit plusieurs fois, ne peuvent pas fonder la science car ils sont trop hétérogènes, puisqu'ils proviennent soit de l'univers du discours logico-méthodologique, soit du discours déontologique et éthique, soit des discours philosophico-ontologique, psychologique ou sociologique. Et Merton, en très grand sociologue qu'il est, s'est vite aperçu de cette incongruité et a écrit un texte (Priorities in Scientific Discovery: A Chapter in the Sociology of Science, in «American Sociological Review», décembre 1957, pp. 635-659) où il dit à peu près ceci: la science est une institution, et comme toutes les institutions humaines, elle charrie des valeurs incompatibles, tout au moins en puissance. Le savant, précisément pour cette raison, doit être modeste et faire preuve d'humilité, mais en même temps il doit être original. Son originalité sera aussi attestée par la primauté de sa découverte. Pour faire valoir et défendre cette primauté, il doit se montrer, obtenir des appuis, se mettre en évidence.

C'est ainsi que passent à la trappe l'humilité et la conscience de ses propres limites, le côté provisoire de la recherche. Dans le monde actuel, pour faire reconnaître la primauté d'une découverte, il faut, surtout dans certaines disciplines, être égotiste, vaniteux, beau parleur, voire télégénique. 
N'y a-t-il pas là un grand paradoxe, une ambiguïté de taille?

Charles Darwin (1809-1882) est informé par sir Charles Lyell (1797-1875), géologue écossais, que lord Russel Wallace (1823-1913), le naturaliste fondateur de la géographie zoologique, est en train de publier une présentation de la théorie de l'évolution. Quelle est la réaction de l'auteur De l'origine des espèces par voie de sélection naturelle (1859)? En gros, elle est la suivante: l'idée qu'il faut publier ma théorie pour faire date, pour en établir la priorité, alors que je dois encore la perfectionner et la mettre au point, m'est insupportable. Cependant, la perspective que quelqu'un publie sur l'évolution avant moi m'est extrêmement désagréable, et même pénible.

Cet exemple montre de façon éloquente la contradiction et l'ambivalence du comportement réel du savant, dont les impératifs institutionnels mertoniens n'arrivent pas à bien en rendre compte. La république des savants est régie par des normes endogènes, mais également par des normes exogènes. Le respect de ces types de normes engendre des comportements qui peuvent être déviants par rapport à l'ethos de la science (universalisme, communalisme, désintéressement et doute systématique), mais tout à fait indispensables au maintien de l'identité et de l'intégrité intellectuelles du savant.

La structure normative de la science renferme, en conséquence, une ambivalence cruciale. Merton n'est pas dupe de la véritable nature de la république des savants, ce qui néanmoins ne l'empêche pas de répéter que le sociologue doit se borner à étudier uniquement et exclusivement les aspects intra-institutionnels de la science, et laisser aux philosophes l'étude des fondements de la science en tant que science, indépendamment des déterminations particulières qui, elles seules, sont l'objet du travail des sociologues.

Les tenants du «programme fort» diront: Merton néglige le fait que la science est hétérogène, qu'elle est un mélange indissociable de social et de cognitif, qu'il est hasardeux de dissocier les problèmes de la recherche de ceux du jeu social, de ses règles, de l'organisation sociale, de ses formes d'apprentissage et de solidarité. Ces deux dimensions de la science sont les deux faces d'une même médaille.

Ces critiques ne me semblent pas très pertinentes: elles présument que la société se fonde sur un modèle mécaniste. C'est ce modèle qui permet ensuite de mêler des aspects qu'il faudrait tenir séparés. En effet, la nature sociale est confondue avec des procédures explicatives, conçues à leur tour comme des propriétés de l'homme et de la société.

La sociologie de la science de Merton, comme par ailleurs toute la sociologie de la science de son époque, a des faiblesses, mais ce ne sont pas celles que dénoncent les tenants du «programme fort». Sans les travaux de Merton, la sociologie de la science n'aurait jamais produit l'ensemble des connaissances que l'histoire de notre discipline atteste de la manière la plus claire.

Les travaux de Merton suggèrent que la science a besoin de liberté et de démocratie. Cependant, ils passent sous silence les rapports de la science avec le capitalisme, et les rapports du capitalisme avec la démocratie. 


\section{TALCOTT PARSONS}

Talcott Parsons (1902-1979) parvient-il à élucider ces questions mieux que Merton ou d'autres sociologues concernés par cette problématique?

Cet auteur, probablement le plus important sociologue, avec Merton, de la deuxième moitié de notre siècle, a abordé les questions qui nous intéressent dans son livre The Social System, paru pour la première fois en 1951.

Ce livre est très difficile, comme tous les grands livres. Mais sa compréhension nous a été facilité par Guy Rocher (Talcott Parsons et la sociologie américaine, Paris, PUF, 1972) et surtout par François Bourricaud (L'individualisme institutionnel. Essai sur la sociologie de Talcott Parsons, Paris, PUF, 1977).

Je pose donc la question: Parsons a-t-il mieux réussi que ses devanciers à élucider les questions en suspens? Une réponse catégorique est difficile à donner.

Parsons considère, d'une certaine façon, que les questions concernant les rapports de la science avec le capitalisme et la démocratie ne sont pas pertinentes. Le seul point vraiment important pour lui concerne l'institutionnalisation de l'enquête scientifique. Celle-ci est devenue une activité très spécialisée et très technique, réservée à des personnes ayant une préparation ad hoc et des compétences particulières. Cette activité est ambiguë et ne peut être orientée de manière univoque. L'exemple de la recherche sur la constitution de l'atome est emblématique à ce propos. Les connaissances produites par la recherche atomique peuvent être employées pour fabriquer des bombes, une centrale électrique ou un appareillage médical.

La spécialisation des rôles, l'impossibilité dans laquelle se trouve la science de se fixer des buts précis et des limites, placent celle-ci dans une position particulière, en font un corps à part dans la structure sociale. La nature technique de la science crée une cassure dans la communication.

Les savants ont besoin de l'argent et du soutien de la société, mais la société n'est pas en mesure de juger ni d'orienter leurs activités. Seuls d'autres savants peuvent le faire, en un langage qui ne peut être entièrement compris que par les spécialistes de la question. Par ailleurs, et je l'ai déjà dit, personne ne peut dire où se situe la frontière entre la science et ses résultats pratiques, ceux que l'homme ordinaire peut apprécier, comprendre et employer. Cela résulte de la structure cognitive de la science: les ramifications que comportent les problèmes scientifiques ne sont jamais directement et immédiatement réductibles aux applications concrètes. C'est pourquoi, on n'a jamais douté de l'utilité de la science fondamentale, même quand ses abstractions paraissaient de purs jeux de l'esprit. Il suffit de penser à ce propos à la théorie de la relativité ou à la force d'inertie.

La rupture dans la communication rend impossibles les contrôles externes de la science. Même lorsqu'un savant se livre à des activités qui paraissent choquantes, il est très difficile d'arrêter son travail. Personne n'est en mesure d'en prévoir ou d'en calculer les implications et les développements, ni de dire s'ils seront bénéfiques ou malfaisants. Même dans les cas douteux, il est dangereux d'intervenir de l'extérieur. Les débats actuels sur le nucléaire, militaire et civil, sur les perturbations de l'écosystème, sur le génome humain, sont éloquents. Nous n'arrivons pas à trouver des réponses plausibles à ces questions, et nous en sommes perturbés. Nous savons que la science fondamentale est en droit - c'est même sa raison d'être - de prospecter tous les champs de l'inconnu et d'explorer l'inexploré. Son inventivité est sans fin. Nous pressentons tout le danger que cela 
recèle, mais que faire? Il est impossible d'arrêter la recherche. Il s'agit donc de faire preuve de vigilance, de contrôler le passage à l'application, aux techniques. Tout ce qui est réalisable ne doit pas pour autant se concrétiser. La société a son mot à dire sur les utilisations qu'on peut faire des découvertes scientifiques. Elle doit et peut les empêcher ou les surveiller; elle doit et peut pondérer les effets d'une réalité. En revanche, il n'est pas de son ressort de déterminer les limites imposables à des virtualités; la fixation de ces limites devrait appartenir aux sociétés savantes, aux organismes de régulation de la science.

L'institution scientifique est alimentée par les ressources publiques et/ou privées. Elle poursuit une activité multiforme et d'une hardiesse extraordinaire, dans le désordre et dans la confusion des idées. Cette activité, qui ne se réduit jamais à l'utilité pure, échappe à tout contrôle extérieur, utilise un langage accessible aux seuls spécialistes, et exige une liberté absolue, une autonomie totale, une auto-direction indéfinie, indéfinissable et illimitée.

On le voit, nous avons ici affaire à une situation unique dans le monde social: sur la base d'une promesse, soit la compréhension rationnelle du monde empirique, sans garantie aucune quant à des applications pratiques, nous avons constitué la science en un monde particulier, en un corps social séparé de tous les autres.

Précisément parce qu'elle constitue un «corps séparé» dans la société, la science peut susciter des craintes, des suspicions, des réactions irrationnelles. Les savants sont tantôt perçus par l'homme ordinaire comme des magiciens bénéfiques, tantôt comme des sorciers dangereux. Cela tient au fait que leurs activités nous sont inaccessibles et qu'elles sont porteuses de nouveau, d'inattendu, dont l'évidence est toujours tardive, et dont les effets sont susceptibles d'être réparés seulement après en avoir usé et mésusé.

L'originalité de Parsons réside dans le fait d'avoir perçu un élément crucial: la société a besoin de la science, la société veut que la science soit utile, qu'elle puisse servir à satisfaire les besoins des hommes, et c'est bien pourquoi elle lui fournit les ressources nécessaires. Mais la société n'a pas la possibilité de contrôler, politiquement, moralement et juridiquement, les activités des scientifiques, leur légitimité, leurs mérites. Seuls les scientifiques eux-mêmes ont le pouvoir de le faire d'une manière raisonnable et convenable, et personne ne peut prévoir les développements ultérieurs d'une recherche présentement avortée. En d'autres termes, Parsons a montré que ce processus d'institutionnalisation a fait de la science un sous-système social gouverné par des règles propres, fonctionnant selon une logique spécifique, un sous-système auquel il est difficile d'imposer des standards utilitaires ou exogènes.

Certes, la société peut fixer des conditions à l'octroi de ressources, elle peut soutenir un type de recherche à l'exclusion de tous les autres, mais même dans ce cas elle n'obtiendra pas l'effet escompté. Bien sûr, elle pourrait stériliser la science si cette dernière produisait des résultats paradoxaux, non voulus, ou pervers par rapports aux intentions initiales, mais les effets d'une telle intervention seraient plus graves que ceux qu'on voudrait éviter.

La thèse de Talcott Parsons mérite d'être un peu nuancée. Elle me semble fondée en ce qui concerne la recherche fondamentale, elle demeure fragile dans le domaine de la recherche appliquée et de la technologie.

Dans les secteurs polytechniques, les liens science-société sont absolus. Les conditionnements auxquels la science est soumise sont très forts; la dépendance du chercheur à l'égard de son commanditaire est totale. Or dans une société où les 
stocks de connaissances fondamentales sont gigantesques, le secteur de la technologie devient prédominant. En effet, il peut compter sur des gisements de savoir très riches, et vivre des rentes produites par l'accumulation des connaissances scientifiques. C'est ainsi qu'en Occident, on a mis en place des programmes nationaux de recherche finalisés à la réalisation de buts déterminés, buts que l'on peut atteindre relativement vite puisque les connaissances fondamentales existent déjà: il suffit de les rendre opératoires.

Depuis deux ou trois décennies, tous les moyens sont mis à la disposition de la recherche appliquée, des recherches utilitaires. Ce phénomène est en train de changer le mode de fonctionnement de la corporation des scientifiques: il est devenu indispensable d'expliquer aux profanes ce que l'on veut faire, d'en montrer l'utilité, d'aller au devant des attentes des commanditaires, de produire des résultats vendables. Les chercheurs deviennent ainsi des managers sachant vendre leur marchandise et bien la présenter. L'ethos décrit par Merton, la liberté pour la science de Parsons, l'égalitarisme scientifique de Ben David s'appliquent de moins en moins à la recherche telle qu'elle se fait sous nos yeux.

Il faut dire, avant de conclure, que tous ces auteurs ont une vision des rapports science-société fortement marquée par les tensions et les conflits entre la science comme institution et la société en tant que système social, entre les besoins du progrès scientifique et les besoins pratiques du système social qu'il faut satisfaire. Ils considèrent tous que la science ne souffre pas d'hétéronomie, qu'elle ne puise pas dans les règles sociales les principes de son action. Elle a ses règles propres, elle jouit d'une autonomie importante et d'une indépendance relative. Elle doit continuer à progresser. La société doit éviter que la technique ne passe aussitôt aux marchands le relais de ses inventions.

Mais peut-elle le faire? Si oui, comment le faire?

La sociologie de Parsons ne donne aucune réponse à ces questions. Pour avoir des réponses, il faudra attendre les analyses très critiques des «nouveaux sociologues », ceux du «programme fort», vis-à-vis de la sociologie classique.

\section{LA «NOUVELLE»SOCIOLOGIE DE LA SCIENCE}

A partir des années ' 70 , des recherches, de plus en plus nombreuses, ébranlent fortement la sociologie de la science classique: elles déstabilisent certains mythes relatifs à la science pure et à la république des savants, et font apparaître la science comme un système de statuts et de rôles socioprofessionnels. Ces recherches mettent en évidence les conditionnements socio-économiques, politiques et culturels et leur impact sur l'organisation de la recherche, sur ses résultats, sur ses méthodes et sur ses objectifs. Elles ouvrent la voie au «programme fort » qui lance une série de défis que nous allons maintenant exposer et examiner de façon analytique.

Avant de commencer, voici tout d'abord quelques indications bibliographiques: M. Ashmore, The Reflexive Thesis. Wrighting the Sociology of Scientific Knowledge, paru en 1989, P.T. Manicas et A. Rosenberg, The Sociology of Scientific Knowledge. Can We Ever Get It Straight? in «Journal for the Theory of Social Behavior», 1988, vol. 18, n. 1, pp. 51-76. Sur la diffusion en France du «programme fort», je vous renvoie aux articles de François-André Isambert (Un 
«programme fort» en sociologie de la science?, « Revue française de sociologie», juillet-septembre 1985, pp. 485-508; Après l'échec du "programme fort», une sociologie du contenu de la science reste-t-elle possible?, in Le relativisme est-il résistible? Regards sur la sociologie des sciences, R. Boudon \& M. Clavelin, éds, Paris, PUF, 1994, pp. 51-76), et aussi à celui de Benjamin Matalon, Sociologie de la science et relativisme, «Revue de synthèse », juillet-septembre 1986, pp. 267290.

La nouvelle sociologie de la science veut démystifier les prétendues autonomie et neutralité de la science et de ses institutions; elle veut faire reconnaître le principe de la pluralité des sciences (la science n'est pas une), et soutient que les méthodes, les procédures et les résultats des enquêtes scientifiques sont des produits culturels qui ont une histoire propre, spécifique. Pour cette nouvelle sociologie de la science, les questions épistémologiques paraissent sans objet véritable. L'épistémologie sacralise la science alors qu'il faut la dépouiller du caractère «exceptionnel» qu'on lui a reconnu jusqu'ici.

Le point de départ du «programme fort» a été formulé par Barry Barnes et David Bloor, entre 1974 et 1982, dans une série d'articles très contestés. Ces deux auteurs se proposent d'attribuer le même fondement, la même légitimité cognitive, à tous les systèmes de connaissance acceptés par la société. La science, le mythe, la religion, la magie n'ont d'autre fondement que la société; leur base est par conséquent foncièrement de nature sociale. Dès lors la sociologie de la science doit respecter les quatre principes que voici:

1. Causalité. Elle doit traiter des conditions de production des différents systèmes de croyance. Aux diverses causes sociales peuvent s'en ajouter d'autres, pourvu que l'explicans ne fasse pas référence à la validité des croyances en question. L'explication doit être, en tous les cas, de type déterministe.

2. Impartialité. Croyances vraies et croyances fausses, rationnelles et irrationnelles, ayant ou n'ayant pas rencontré un succès pragmatique, doivent toutes être expliquées par la sociologie de la connaissance scientifique, à partir du moment où elles sont admises ou reconnues par une collectivité.

3. Symétrie. Les croyances vraies et les croyances fausses, rationnelles ou irrationnelles, efficaces ou inefficaces, doivent être expliquées par le même type de causes.

4. Réflexivité. Les mêmes schémas explicatifs doivent être appliqués à tous les domaines de la sociologie de la connaissance et à la sociologie de la connaissance elle-même.

L'explication causale, l'impartialité, la réflexivité, la symétrie caractérisent l'approche naturaliste, scientifique, de l'étude des systèmes de connaissance, et plus particulièrement des connaissances scientifiques.

Bloor rejette la critique selon laquelle il existe une connaissance ordinaire, propre au sens commun, et une connaissance scientifique, produit d'une rupture avec ce sens commun. Pour lui, il s'agit d'un parti pris, d'un préjugé de nature déductif, rendant impossible l'explication d'une action ou d'une conduite que nous ne pouvons pas déduire de principes prédéfinis. Une approche qui procède 
par induction ou résulte de certains indices à des faits qu'ils rendent plus ou moins probables, rend plausibles des modes d'acquisition de modèles scientifiques non formalisés ou implicites, et permet de les transférer ou de les appliquer ailleurs. Le «programme fort» considère, en somme, que la connaissance scientifique peut être assurée grâce à l'analogie et à l'extension métaphorique de modèles conceptuels déjà existants et fondés socialement.

Si tout système de connaissance est produit par des causes sociales, doit-on alors considérer que ces dernières sont toujours incontestables et vraies?

Le principe de symétrie de l'explication causale écarte cette question qui concerne, en réalité, la vérité et la fausseté des croyances expliquées.

Pour Bloor, affirmer qu'une croyance est vraie ou fausse n'a rien à voir avec le fait qu'elle soit ou ne soit pas déterminée par une cause relevant de facteurs sociaux. L'explication causale est valable en soi et pour soi, indépendamment des facteurs auxquels elle s'applique.

Par ailleurs, ces facteurs peuvent être multiples, et différents les uns des autres. La connaissance, dès lors, est celle que les acteurs sociaux, déterminés historiquement, culturellement et du point de vue de la structure sociale, définissent comme telle. Le relativisme est une nécessité et le scientisme un lieu commun.

Ce point de la doctrine de Bloor, tel que vous pouvez le lire dans son livre Socio/logie de la logique ou les limites de l'épistémologie, paru en 1976 et traduit en français en 1983, n'est rien d'autre que la question de l'auto-fondation logicoformelle de la science et du contrôle qu'elle exerce sur la validité de ses propres produits.

Bloor parle à ce propos de circularité du discours scientifique. Par là, il entend que:

1. La science n'arrive pas à s'auto-fonder avec ses propres procédures.

2. La science ne peut pas s'auto-décrire de façon cohérente. Les critères qu'elle propose ne permettent pas de distinguer la science de la non-science. Par ailleurs ces critères sont fluctuants et variables.

3. La science et les méthodes qui la caractérisent se fondent sur des valeurs. Ils sont donc équivalents, en ce qui concerne les fondements de leur validité, à d'autres univers cognitifs fondés sur des valeurs différentes.

Ces trois points donnent pour acquise, selon Bloor, la variabilité du concept de rationalité.

4. La «theory adhocness »: le choix entre des théories rivales n'est jamais neutre. Les théories font partie d'un contexte et s'en nourrissent. Lorsque le contexte change, la signification des termes d'une théorie change aussi. La confrontation entre les termes de deux théories est dénaturée, car ces termes n'ont pas la même portée ni le même poids.

Essayons très rapidement de voir de plus près cette question des cercles logiques de l'auto-fondation logico-formelle de la science. Barnes et Bloor en parlent dans un article de 1982 intitule Relativism, Rationalism and the Sociology of Knowledge et publié dans le volume édité par M. Hollis et S. Lukes, Rationality and Relativism (Oxford, Blackwell, 1982, pp. 21-47). 
Dans cet article, les deux auteurs affirment catégoriquement ceci: «L'objectif rationaliste de produire des éléments de connaissance ayant une fiabilité universelle et étant, au même moment, justifiés de façon indépendante du contexte, cet objectif est irréalisable».

Pourquoi? Je laisse de côté ici la question de la fiabilité (qu'est-ce que cela signifie-t-il? Est-ce l'aptitude d'une construction théorique à fonctionner sans incidents pendant un temps donné? Quelles en sont les limites?) ainsi que l'autre question relative à la justification des objectifs, étant donné que Bloor et Barnes n'en tiennent aucun compte, sans par ailleurs en donner des raisons plausibles. Je me borne donc, ici, à suivre les arguments que nos deux sociologues avancent, en se fondant surtout sur la logique et les mathématiques.

Il faut dire tout d'abord que soit la logique, soit les mathématiques, étudient des objets de Raison, ou concepts, qui ne sont ni des objets physiques ni des objets psychologiques. Ces objets de Raison possèdent une propriété très importante: l'objectivité, qui est indépendante de nos sensations et de nos représentations mentales, mais pas de notre Raison. Or il faudrait nous dire quelle est la nature de cette Raison, et surtout des objets qui satisfont à l'objectivité. À ce propos, les travaux de Bloor ou de Barnes (Scientific Knowledge and Scientific Theory, London, Routledge and Kegan Paul, 1974, et Interests and the Growth of Knowledge, London, Routledge and Kegan Paul, 1977) restent muets. En effet, la question de l'objectivité de la connaissance mathématique n'est jamais abordée dans ces études.

David Bloor a publié deux textes importants pour notre sujet. Le premier est l'article Wittgenstein and Mannheim on the Sociology of Mathematics (in «Studies in the History and Philosophy of Sciences», 4, 1973, pp. 173-191) et le deuxième est le livre Knowledge and Social Imagery (London, Routledge and Kegan Paul, 1976), traduit en français sous le titre Socio/logie de la logique. Les limites de l'épistémologie (Paris, Pandore, 1983).

Bloor écrit à la page 118 de ce livre: La mathématique «ne rend pas compte du caractère inéluctable de ses déductions. Elle n'explique pas pourquoi il semble que les conclusions mathématiques ne puissent être différentes de ce qu'elles sont. Il est exact que les situations types ont une sorte de pouvoir physique. On ne peut arranger et classer les objets à sa guise. Ils ne feront jamais tout ce qu'on souhaite et, dans cette mesure, ils s'imposent effectivement à nous. Mais ceci ne leur confère pas pour autant une quelconque autorité. Nous restons libres d'imaginer qu'ils puissent se comporter autrement, alors que nous n'éprouvons pas cette liberté vis-à-vis des mathématiques. Il y a donc une similitude entre l'autorité logique et l'autorité morale. Seulement, l'autorité est une catégorie sociale...», et c'est bien cette «composante sociale» qui explique «la sélection parmi les modèles physiques et leur aura d'autorité».

Dès lors il s'agit de «montrer comment et pourquoi les gens pensent ainsi qu'ils le font; comment les pensées naissent, comment elles acquièrent, conservent et perdent les statuts de connaissances ». En d'autres termes, il est indispensable de «faire la lumière sur le comportement des hommes, le fonctionnement de leur esprit et la nature de l'opinion, de la croyance et du jugement». Toujours d'après Bloor, nous ne pouvons pas y parvenir sauf si nous arrivons à montrer préalablement «comment les mathématiques s'édifient à partir d'éléments naturalistes: expériences, processus psychologiques, tendances naturelles, habitudes, 
modèles de comportement et institutions. Pour cela, il faut pousser l'étude au-delà du résultat des réflexions humaines : il faut dépasser le produit et étudier les opérations de production ». En conséquence, toujours selon Bloor, «les idées relatives à la connaissance sont fondées sur des représentations sociales», "la nécessité logique est une forme d'obligation morale», «l'objectivité est un phénomène social».

La science ne peut pas démontrer ses fondements, elle ne peut pas tirer d'eux sa raison d'être, ni non plus ses connaissances et ses préceptes. Les fondements de la science sont des croyances. Or «Toutes les croyances doivent être expliquées de la même manière, quelle que soit l'évaluation qui en est faite». «Rien n'est absolu ni définitif». «Toute connaissance est conjecturale et théorique, mais aussi relative à la situation de celui qui la produit; aux idées et aux conjectures qu'il est en mesure d'émettre; aux problèmes qui le préoccupent; aux effets réciproques des hypothèses et des critiques dans son milieu; à ses buts; à ses expériences; aux normes qu'il applique et aux significations qu'il utilise. Quel rôle tous ces facteurs jouent-ils, sinon de déterminer de façon naturaliste les croyances, qui pourront ensuite être étudiées sur un plan sociologique et psychologique? (...) L'explication entière est une conjecture, même si c'est une conjecture à propos d'autres conjectures ».

Relativisme méthodologique? Certainement. Mais peut-on affirmer que ce problème des fondements est aussi essentiel que Bloor le présume? Rappelonsnous du théorème de Kurt Gödel (1906-1978) et de son célèbre paradoxe: soit une théorie basée sur un nombre fini d'axiomes assez riches pour permettre la construction de l'arithmétique. On peut toujours trouver une proposition indécidable (une relation qui n'est ni vraie ni fausse) dans la théorie. Si l'on ajoute $a$ priori, comme axiome à la théorie, que cette proposition paradoxale est vraie, on a alors une autre théorie, mais dans laquelle une nouvelle proposition est à son tour indécidable. On voit qu'il est exclu qu'un nombre fini d'axiomes suffise à créer quelque chose d'absolument universel, c'est-à-dire où est vrai à la fois le principe du tiers exclu, et la certitude que toute proposition soit vraie ou fausse.

Il y aura toujours des théorèmes indémontrables parce que dépourvus de réponses. Est-ce un motif suffisant pour tout rejeter? Pour dire que l'argumentation scientifique est de la même veine que celle des astrologues ou des chiromanciens?

Le véritable problème me semble se trouver ailleurs: non pas dans le fait de construire des systèmes cognitifs inconditionnellement valides et capables de s'autofonder de façon complète, mais plutôt dans le fait de respecter le principe de non-contradiction, de construire des systèmes valides conditionnellement à la validité des autres. Lorsqu'on accepte que la notion de validité puisse se rapporter à n'importe quel système de croyances, qu'elle soit fonction du contexte social, nous n'avons alors aucune possibilité de contrôler empiriquement la dépendance des systèmes de croyances admis par leur contexte social.

De plus, en réduisant la validité à la crédibilité sociale, nous ne pouvons plus distinguer le contexte de la validation ou de la justification du contexte social d'origine ou d'acceptation. Nous ne pouvons plus distinguer l'usage de certaines règles et de certaines pratiques qui nous permettent de décider de la vérité/validité/rationalité de certaines propositions, de l'attribution de la crédibilité aux règles et aux décisions qui en résultent. Il n'y a plus, dans ce cas, aucun moyen de faire de l'expérimentation mentale, d'explorer les systèmes de règles syntactiques 
ou sémantiques. Ceci me paraît grave, car alors nous nous privons volontairement d'un critère essentiel pour bien aborder la question du démarquage de la science de la non-science.

Mais avant d'aller plus loin, je dois ouvrir une courte parenthèse sur David Bloor, philosophe et mathématicien reconverti à la sociologie et considéré comme le chef de file du «programme fort».

Bloor est convaincu que la sociologie peut dire le mot de la fin sur tous les processus cognitifs. Il a écrit à ce propos: «La sociologie de la connaissance peutelle étudier et expliquer la nature et le contenu de la connaissance scientifique? De nombreux sociologues pensent que non. Selon moi, ils trahissent ainsi leur position de sociologue... En fait, il n'existe aucune limite dans le caractère prétendument absolu ou transcendant de la connaissance scientifique, ou dans une quelconque notion particulière de la rationalité, de la validité, de la vérité ou de l'objectivité».

La science et son développement, pour Bloor, ne sont pas gouvernés par leur propre rationalité ni par le rapport entre l'expérience, la logique et le langage. Il n'y a aucune détermination interne, ni non plus une rationalité en soi et pour soi. Tout est déterminé par la vision du monde que le groupe social possède. Dès lors, la corrélation entre le niveau de développement économique et celui des théories scientifiques me paraît inévitable. Ces dernières étant fortement influencées par les idéologies.

Pour Bloor, la science est une croyance du même ordre que les croyances religieuses. Lorsqu'il y a consensus dans un groupe social à propos d'un objet déterminé, cet objet devient, en certaines circonstances, une croyance. Par ailleurs, la science, d'après Bloor - faisant ici référence explicite à Durkheim (Durkheim and Mauss Revisited. Classification and the Sociology of Knowledge, in «Studies in History and Philosophy of Science», 13, 1982, pp. 267-297) -, a la même nature que la religion; elle reçoit, en effet, son autorité du «pouvoir de la force collective», des «puissances extérieures», des «principes qui organisent la société » (lire en la matière Dénes Némedi, Durkheim and the «Strong Programme» in the Philosophy of science, in «Revue européenne des sciences sociales», XXVIII, 1990, n. 88, pp. 55-75).

Ici, nous avons affaire à une contradiction de taille: d'un côté les quatre principes de Bloor (explication causale de toutes les croyances, impartialité à l'égard de la vérité et de la fausseté, symétrie et réflexivité) présupposent l'existence d'une nature précédant la science et la religion et nous imposant de traiter ces dernières de manière équivalente; de l'autre côté, si la science est l'expression des structures sociales, économiques et politiques, elle ne peut pas être fondée sur la nature: elle n'est, dans ce cas de figure, que l'extériorisation de la société et de ses principes d'organisation. En conséquence, elle est l'expression de la société.

Dans un cas comme dans l'autre, Bloor évite de nous fournir les démonstrations, en fait et en droit. Nature et science sont réifiées. Qu'est-ce que la nature humaine? Il y en a-t-il vraiment une? D'où pourrait venir cette caractéristique grâce à laquelle «Notre pensée, dans son ensemble, suppose instinctivement que nous existons dans un environnement extérieur commun possédant une structure déterminée »? Peut-on affirmer que la connaissance scientifique est affaire de consensus ou d'élaboration collective? Et que ce consensus et cette élaboration collective sont pareils à ceux de la religion? Où introduire l'explication causale? Est-elle valable en toutes circonstances, même là où la validité est relative? 
En admettant «le présupposé que l'homme peut systématiquement réagir au monde par l'intermédiaire de son expérience», je suis obligé de reconnaître que les jugements de réalité sont fondés sur des expériences élaborées socialement. Dans ces conditions, les conventions de la science ne sont ni absurdes ni arbitraires.

Il me reste encore à dire quelques mots sur le noyau dur du programme de Bloor que l'on peut synthétiquement formuler ainsi : toute explication scientifique doit être causale.

Le sociologue anglais fait découler sa démonstration d'un postulat primordial ainsi formulé: «la vérité, la rationalité et la validité » sont «des buts naturels de l'homme »; « elles orientent certaines tendances naturelles ». L'homme « raisonnerait naturellement de façon juste et se rallierait à la vérité logique lorsque celle-ci se présenterait à lui ». Ce postulat a un sens, pour autant qu'on présume l'existence d'une nature humaine. Cependant, si l'on dit que l'expérience est le produit d'influences et de facteurs sociaux, la rationalité n'est plus un élément constitutif de la nature humaine, elle n'est qu'une construction sociale parmi d'autres; à moins d'imaginer qu'une procédure explicative de type mécaniste puisse constituer la nature humaine.

Il y a là une faiblesse conceptuelle très importante, une fragilité argumentative et démonstrative si considérable que toute la construction de Bloor n'offre plus les garanties de rigueur indispensables aux élaborations théoriques.

La quête d'une théorie des fondements recèle, surtout dans les sciences sociales et historiques, davantage de risques qu'elle ne procure de bonnes hypothèses et de réponses plausibles. Malheureusement la construction de Bloor n'échappe pas, elle aussi, à ce truisme vétuste et, bien que caduc, toujours en circulation dans le domaine des philosophies relativistes.

\section{L'ANTHROPOLOGIE DES SCIENCES ET DES TECHNIQUES}

Examinons maintenant, à titre d'exemple paradigmatique, les travaux du représentant français le plus connu du courant le plus radical du «programme fort», «l'Actant Network School», «l'acteur-réseau», pour lequel aucune distinction, soit-elle analytique ou épistémologique, n'est possible entre les catégories d'objets et les facteurs constitutifs de l'activité scientifique: «technical, social, political and economic factors are inextricably bound together».

Le chercheur français le plus représentatif de ce courant de «l'acteur-réseau» est Bruno Latour, professeur à l'Ecole nationale supérieure des mines de Paris, né en 1947, agrégé de philosophie, auteur de plusieurs livres et articles. Parmi ses livres, mentionnons Les microbes (1984), La vie de laboratoire (1988), La science en action (1989), Nous n'avons jamais été modernes (1991), et bien entendu l'anthologie de la science sociale de langue anglaise, dont le premier volume est intitulé La science telle qu' elle se fait (Paris, La Découverte, 1991) et le deuxième volume Les scientifiques et leurs alliés (Paris, Pandore, 1985). Les dates que je donne ici sont celles des dernières éditions.

La vie de laboratoire, écrit en collaboration avec Steve Wolgar et publié en anglais en 1975, a été traduit en français en 1988. Il s'agit d'une monographie sur 
le laboratoire de biologie moléculaire (neuro-endocrinologie) du professeur Roger Guillemin, prix Nobel de médecine en 1978, laboratoire rattaché au Salk Institute for Biological Studies, à La Jolla, en Californie. Il s'agit d'un texte où les distinctions entre les diverses catégories d'objets et d'acteurs servent à donner un sens au réel, au monde du laboratoire finement analysé. Les descriptions et la narration, respectueuses même de l'ordre temporel des événements, sont des plus traditionnelles nonobstant les professions de foi radicales, prétendument nouvelles et contre-intuitives, en méthodologie de la recherche scientifique.

Bruno Latour a passé deux ans dans le Laboratoire de neuro-endocrinologie de l'Institute Salk, comme un anthropologue l'aurait fait dans une tribu primitive, et c'est là, selon ses propres déclarations, qu'il a commencé à élaborer son ethnographie réflexive des sciences, et à mettre au point ses analyses matérialistes de la production de la science.

Bruno Latour et Steve Wolgar décrivent dans ce livre les tâtonnements, les hésitations, les parades ainsi que les tactiques et les stratégies des chercheurs de l'équipe du professeur Roger Guillemin.

L'activité scientifique de ces chercheurs porte-t-elle sur «la nature», sur la «découverte d'une réalité cachée et préexistante»? Assurément pas. Ces chercheurs fabriquent, transmettent, diffusent des inscriptions, des énoncés, des «statements», constituent des réseaux, les modélisent de diverses manières, pour ensuite faire émerger des «faits », des réalités tenues pour indiscutables, capables de résister aux objections les plus fortes, aux attaques les plus farouches des chercheurs concurrents. En d'autres termes, ils fabriquent des «acteurs-réseaux», négocient des «associations», travaillent à des «enrôlements».

Latour suit pas à pas la fabrication de ces faits: de l'hypothèse à la constitution de l'objet, de la phase expérimentale à celle du faire-valoir rhétorique, et cela au milieu de conflits et de soucis propres à la carrière de chaque chercheur, aux rivalités entre les laboratoires.

Les chercheurs doivent s'acquitter d'une seule tâche: fabriquer les faits et les rendre crédibles malgré la résistance et l'opposition des chercheurs des laboratoires rivaux et concurrents. Ce travail de construction du fait scientifique est foncièrement agoniste. C'est une entreprise conflictuelle, et le conflit conditionne l'argumentation et la matérialisation du fait en réalités extérieures, antécédentes aux activités de recherche.

Un collaborateur de Latour, Michel Callon, nous a apporté des éclaircissements à ce propos et de façon plus explicite, moins sibylline. Dans son introduction au volume La science et ses réseaux. Genèse et circulation des faits scientifiques (Paris, La Découverte, 1989), il présente ce dossier mystérieux portant sur les rapports entre les objets et les agents, sur le rôle des divers facteurs scientifiques, technologiques et sociaux.

Callon nous dit à peu près ceci: un fait scientifique n'est ni un constat, ni un témoignage. C'est une construction, élaborée et diffusée dans un certain espace (le laboratoire et ses réseaux d'irrigation). «Pour décrire la fabrication d'un fait scientifique, c'est-à-dire le double mouvement par lequel il est construit et trouve des débouchés, il faut donc analyser les réseaux qu'il noue et sans lesquels il serait vidé de tout contenu et de tout avenir ». Dès lors, «Les deux propriétés qui caractérisent le fait scientifique - la capacité de résister à la critique et la faculté d'intéresser d'autres acteurs (collègues, utilisateurs) - ne lui appartiennent pas en 
propre: elles lui sont attribuées par les réseaux négociés et mobilisés pour le construire et pour lui fournir un espace de circulation».

Déconstruire le fait revient à reconstituer le mode de production du laboratoire et à mettre en évidence les réseaux dans lesquels il est situé. «La fabrication des faits est un processus continu, qui consiste en la mobilisation progressive, tâtonnante et négociée, de réseaux auxquels ils doivent in fine leur robustesse et la mesure de leur universalité (relative). La séparation entre création et objectivation n'est possible qu'après coup, lorsqu'il s'agit de donner un nom et un statut à une opération réussie».

Un fait scientifique a donc toujours la forme d'un réseau, il n'est jamais le résultat d'une décision rationnelle, émanant d'un esprit libre et sans attaches partisanes. Un fait scientifique reste toujours le produit d'un ou plusieurs réseaux sociotechniques. Il est impur par nature, composé d'éléments hétérogènes et construit de bric et de broc. Et Michel Callon achève ainsi d'élucider ce problème: la science a deux impératifs, résister et intéresser, «éprouver la résistance d'une proposition, mesurer l'intéressement qu'elle suscite». Or cela suppose l'existence d'un espace public de discussion, «au cours de laquelle se jugent et la robustesse des faits et leur intérêt». Cela suppose également «la construction préalable de cet espace de discussion auquel nous avons choisi de donner le nom de réseau sociotechnique ».

En mettant ensemble les facteurs scientifiques et extra-scientifiques, les idéologies, les intrigues académiques et les éléments macro-institutionnels, la construction de la connaissance scientifique est réduite au «processus par lequel les scientifiques donnent un sens à leurs observations », bref elle n'est rien d'autre qu'une activité sociale. N'y a-t-il aucune correspondance entre la nature humaine et l'esprit? La réponse de Latour est sans appel; triviale et évidente: «... la chose et l'énoncé correspondent pour la simple raison qu'ils ont la même origine. Leur séparation n'est que l'étape finale du processus de leur construction».

Et qu'en est-il de l'efficacité du fait scientifique en dehors de la science? Là non plus Latour n'a pas la moindre hésitation: «N'est-il pas extraordinaire qu'une structure peptidique découverte en Californie fonctionne dans le plus petit hôpital d'Arabie Saoudite! Elle ne marche que dans des laboratoires cliniques bien équipés. Considérant que le même ensemble d'opérations produit les mêmes réponses, il y a peu de raisons d'être émerveillé: avec les mêmes tests, on produira le même objet». Latour devient encore plus catégorique lorsqu'il affirme: «... il importe de s'abstenir d'invoquer la réalité extérieure ou le caractère opérationnel de ce que produit la science pour expliquer la stabilisation des faits, parce que cette réalité et cette opérationnalité sont la conséquence et non la cause de l'activité scientifique».

D'une telle vision dérive que «fait» et «artefact» (effet parasite produit par une cause secondaire non contrôlée, par la méthode ou l'observation elle-même, sans que l'expérimentateur en soit toujours conscient, selon la définition donnée par Pierre Bourdieu) ne se différencient que par la présence ou l'absence de la référence aux conditions de construction. De plus, la réalité d'un fait n'existe pas au départ; elle est la conséquence de toutes les opérations susceptibles de lui attribuer «la crédibilité scientifique».

Latour le répète dans le chapitre L'ordre créé à partir du désordre: «... l'activité scientifique est faite de construction et de la défense de points de vue d'abord 
fonctionnels qui sont parfois transformés en objets stabilisés ». Et dans le même chapitre, Latour résume toute sa conception en six thèses:

1. La construction. Il s'agit du processus matériel par lequel les énoncés (statements) «se superposent et les descriptions sont maintenues et réfutées... La discussion entre chercheurs transforme quelques énoncés en pur produit de l'imagination d'une subjectivité individuelle, d'autres en fait de la nature. La réalité n'est que la conséquence du règlement d'une dispute plutôt que sa cause $»$.

2. Le caractère agoniste du champ scientifique. Puisque l'activité scientifique porte sur des opérations sur les énoncés, et pas du tout sur la nature de la réalité, elle est faite essentiellement de controverses, de rapports de force et d'alliances. «La solidité de l'argument joue nécessairement un rôle pour déterminer celui des arguments qui a la plus grande force de conviction».

3. La matérialisation ou réification du fait scientifique. «Une fois qu'un énoncé a été stabilisé dans le champ agonistique, il est réifié et s'intègre aux savoirfaire tacites ou à l'équipement en matériel d'un autre laboratoire».

4. La crédibilité. «La notion de crédibilité permet de lier une chaîne de concepts, comme l'accréditation, les références professionnelles (en anglais credentials) et le crédit accordé aux croyances («credo», «crédible») et aux comptes à rendre («rendre compte de ses actes», «compte rendu», «crédit sur compte»). Cela fournit à l'observateur une vision homogène de la construction des faits et brouille les divisions arbitraires entre les facteurs économiques, épistémologiques et sociologique».

5. Les circonstances. La science est entièrement le produit des circonstances, et par des pratiques spécifiques et localisées elle "paraît échapper à toutes les circonstances ».

6. Le rapport du signal au bruit. Il y a un marché de l'information fonctionnant sur la base d'un bien symbolique, la crédibilité, «toute diminution du bruit de l'opération d'un acteur élève la capacité d'un autre acteur de faire décroître le bruit ailleurs ».

Entre ces six thèses, que Latour appelle «concepts », il y aurait des relations causales d'ordre mécanique. En effet, en mettant sur le marché des énoncés capables de montrer que tous les autres énoncés en la matière contiennent moins d'informations, la crédibilité des premiers doit croître nécessairement, augmenter sûrement. Latour écrit à ce propos: «Si la force de conviction est suffisante, les autres cesseront d'élever des objections, et l'énoncé acquerra un statut de fait. Au lieu d'être le pur produit de l'imagination (subjective), il deviendra une chose objective réelle, dont l'existence ne saurait plus être mise en doute».

En d'autres termes, toujours selon Latour, «L'opération de construction d'information transforme ainsi tout ensemble d'événements inégalement probables. En même temps, cette opération fait appel aux activités de persuasion (agonistique) et d'écriture (construction) pour augmenter le rapport signal/bruit». Pour rendre plus probable un énoncé parmi les autres énoncés également probables, 
«La technique la plus fréquemment employée par les chercheurs est celle d'augmenter ce qu'il en coûte aux autres d'atteindre des alternatives également probables ». Et comment fait-on cela? En incorporant les arguments antérieurs dans une sorte de boîte noire. Dès lors, «le coût des formulations alternatives devient prohibitif; ... tout ce qui a été accepté, quelle qu' en soit la raison, sera réifié de façon à augmenter le coût des objections qui pourraient être soulevées...; l'ensemble des énoncés considérés comme trop coûteux à modifier constitue ce que l'on entend par réalité. L'activité scientifique ne porte pas 'sur la nature', c'est une lutte acharnée pour construire la réalité. Le laboratoire est le lieu de travail et l'ensemble des forces productives qui rendent cette construction possible. Chaque fois qu'un énoncé est stabilisé, il est réintroduit dans le laboratoire (sous forme d'une machine, d'un inscripteur, de savoir-faire, de routine, de préjugé, de déduction, de programme, etc.) et il est utilisé pour creuser la différence entre les énoncés. Ce qu'il en coûte de remettre en cause l'énoncé réifié est si élevé que c'en est impossible. La réalité est alors sécrétée ».

En transformant des énoncés équiprobables en énoncés inégalement probables, les scientifiques créent l'ordre à partir du désordre. Et les conclusions de Latour deviennent ainsi inévitables: dans l'activité scientifique, le social et le technique sont confondus, il n'y a pas de distinction de nature entre les faits et les artefacts, entre les facteurs externes et les facteurs internes, entre le sens commun et le raisonnement scientifique. Il est aussi indiscutable «que la façon dont les chercheurs se définissent en tant qu'individus résulte des conflits d'appropriation qui ont lieu au sein du laboratoire».

Après cette présentation succincte de La vie de laboratoire, il est nécessaire d'ajouter que l'approche de Latour est très radicale, plus radicale encore que celle proposée par Bloor et Barnes, et aussi plus extrême que celle du Richard D. Whitley de Social Process of Scientific Development.

Suivant l'approche de Latour, le scientifique construit l'objet scientifique, mais pour faire croire à sa réalité extérieure, il doit ingénieusement masquer les opérations constructrices. Il doit pratiquer l'illusionnisme et il est lui-même victime de ses propres illusions. Il est constamment animé d'une seule ambition: faire croire à ses illusions.

Dans ces conditions, tout dépend du talent du chercheur: le fait apparaît dans la pensée de ce dernier, mais après sa conception et après l'élaboration des dispositifs expérimentaux. C'est étrange mais c'est ainsi.

Nous avons affaire à une situation proprement bizarre: il n'y a pas de réalité antérieure, il faut la construire, la mettre en ordre, la rendre crédible. Mais il faut postuler cette réalité extérieure comme si elle était effectivement présente. En conséquence, le chercheur vit constamment dans l'illusion, et dans une illusion qu'il a lui même fabriquée. Tout est illusion, la seule réalité est la construction, et pourtant tous, les constructeurs eux-mêmes, croient à la réalité extérieure. Ils sont les premières victimes de l'illusion qu'ils ont eux-mêmes fabriquée.

Si tout est illusion, comment se fait-il que certaines de ces illusions durent si longtemps et qu'elles puissent résoudre tant d'énigmes intellectuelles et de difficultés pratiques? Si la science a ses fondements dans la force sociale des individus et des groupes, dans les institutions, comment se fait-il que Galilée (1564-1642) ait eu raison de la science de son époque et des contraintes de l'Eglise catholique? 
Comment se fait-il que la classification périodique des éléments chimiques du russe Mendéléiev ait pu s'imposer toute seule, sans l'aide de groupes ou d'institutions?

Dans cette approche de Latour, il y a un certain nombre de truismes, la mise en valeur de certaines évidences indubitables, mais aussi la fâcheuse tendance à radicaliser toutes les thèses, à vouloir construire la sociologie de la science sans avoir préalablement posé des fondations solides à l'édifice.

Suite à La vie de Laboratoire, paru pour la première fois en 1979, Latour publie Les microbes. Guerre et paix, suivi de Irréductions (Paris, Métailié, 1984). Dans ce dernier volume, il montre comment, entre 1870 et 1914, les doctrines de Louis Pasteur ont été perçues et reçues par les scientifiques et les médecins, de quelle façon les pasteuriens sont arrivés à les rendre convaincantes et crédibles, et les modalités grâce auxquelles la bactériologie et la société française se sont transformées en parallèle. Ce livre est passionnant surtout à cause de l'habileté avec laquelle l'auteur met en scène les implications politiques de la prophylaxie de la rage et de l'asepsie. Mais le parti pris de Latour de contester la division science/ technique, société/nature, d'attaquer la distinction entre la dimension politique de la science et la vérité de cette même science, de fondre ensemble les rapports de raison et les rapports de force, nous prive d'un certain nombre d'outils fort utiles et nous oblige à travailler à mains nues, dans une obscurité totale, à l'intérieur d'une situation où tout n'est que «croyance dans l'existence du monde moderne, croyance dans l'existence de la logique, croyance dans la présence de la raison, croyance aussi dans la croyance elle-même et dans le savoir tout aussi bien ».

Dans La science en action (Paris, La Découverte, 1989), Latour perfectionne ce modèle d'interprétation et en fait une sorte d'herméneutique généralisée de l'activité scientifique. À la fin de ce livre, écrit dans le but de vulgariser ses doctrines, il résume et présente les règles de sa méthode, en les appuyant sur une foule d'exemples, ainsi: (a) il faut étudier la science en action et non celle déjà achevée; (b) il ne faut pas rechercher les qualités intrinsèques d'un énoncé, mais s'attacher avant tout à reconstituer l'ensemble des transformations qu'il a subi; (c) le règlement d'une controverse est la cause d'une représentation stable de l'état de nature, et non sa conséquence; (d) le règlement d'une controverse est la cause de la stabilité de la société; c'est pourquoi cette dernière ne peut pas expliquer pourquoi et comment une controverse a été résolue; (e) il faut étudier simultanément le partage intérieur/extérieur construit par les différents acteurs des techno sciences; (f) il faut étudier l'angle et la direction du déplacement de l'observateur et la longueur du réseau qui se construit ainsi, sans se préoccuper de la logique ou des structures de la société; $(\mathrm{g})$ il ne faut attribuer aucune qualité particulière à l'esprit scientifique, mais s'intéresser uniquement aux «nombreuses façons dont les inscriptions sont regroupées, combinées, liées entre elles et renvoyées. C'est seulement si quelque chose reste inexpliqué une fois que nous aurons étudié les réseaux longs que nous pourrons commencer à parler de facteurs cognitifs ».

Bruno Latour tire de sa très longue enquête les six principes suivants:

1. La qualité des faits et des machines est la conséquence des transformations opérées par une multitude d'acteurs et de réseaux.

2. Les chercheurs parlent au nom d'alliés qu'ils ont généré et recruté.

3. Il n'y a pas de science, mais des associations d'humains et de non humains. 
4. «Plus le contenu de la science et de la technique est ésotérique, plus elles s'étendent loin à l'extérieur».

5. «L'irrationalité est une accusation qui est toujours portée par celui qui construit un réseau contre quelqu'un qui se dresse sur son chemin ».

6. L'histoire des technosciences est l'histoire des ressources «rassemblées le long des réseaux pour accélérer l'inscription, la mobilité, la fiabilité, la capacité de combinaison et la cohésion des traces qui rendent possible l'action à distance ».

La science en tant que construction sociale totale? Latour est-il, enfin, arrivé à boucler la boucle?

Trop de questions restent en suspens. Essayons de voir si nous arrivons à trouver quelques amorces de réponses dans l'introduction du livre La science telle qu'elle se fait. Anthologie de la sociologie des sciences de langue anglaise (Paris, La Découverte, 1991, pp. 7-36) que Latour a signée avec Michel Callon, et surtout dans son essai de nature plus philosophique Nous n'avons jamais été modernes. Essai d'anthropologie symétrique (Paris, La Découverte, 1991).

Que la nature ne parle pas d'elle-même, que les faits scientifiques soient construits, que la connaissance ne soit jamais une photographie ni un simple enregistrement des résultats fournis par les expériences, ce sont là des affirmations qui ont toujours été admises par les chercheurs non positivistes.

Que la science soit immergée dans la société et l'histoire, voilà une thèse difficilement contestable, comme l'est également le constat suivant lequel l'énoncé scientifique est redevable aux techniques et aux dispositifs matériels et institutionnels, sociaux et cognitifs.

Cependant les choses me paraissent se compliquer terriblement à partir du moment où Latour ne fait plus aucune différence entre le contexte de la création et celui de la justification, et à partir du moment où il postule que la démarche scientifique est assurée exclusivement par le principe de symétrie (entre objets animés et inanimés, entre nature et société, etc.), qu'il est impossible de distinguer le social du technique, que les constructions analytiques ne sont pas justiciables de l'analyse empirique.

Pourquoi considérer que la science en train de se faire est plus importante que la science déjà faite? Pourquoi juger la science exclusivement à partir des modalités de son émergence et de sa production?

Je crois que l'étude du processus des connaissances ne nous dit rien sur la validité ultime d'une théorie. Cette validité est assurée avant tout par le temps et la durée, et par les individus vivant bien après la construction de l'énoncé. Traiter symétriquement des activités dont les statuts fonciers diffèrent, équivaut à faire courir ensemble des chevaux de poids différents, sans handicaps et sur une même distance.

Latour affirme que l'asymétrie suppose une «commune mesure qui permet de déprécier une thèse et d'établir l'autre comme étalon», alors que la symétrie indique qu'il faut expliquer comment on est parvenu à faire le partage entre l'échec et le succès d'une théorie. Ce point de vue (cf. B. Latour, Les «vues» de l'esprit. Une introduction à l'anthropologie des sciences et des techniques, in 
«Culture technique», vol. 14, 1985, pp. 4-30) serait acceptable si nous étions dans le même espace vectoriel et si l'échec et le succès étaient comparables.

Or nous sommes très loin de ce cas de figure. Ce n'est certes pas avec le principe de symétrie qu'on arrivera à expliquer comment une idée, un point de vue, un objet technique se sont imposés et d'autres non.

Il est évidemment très important de savoir ce qu'on arrive à faire dans le laboratoire, mais il est tout aussi important de connaître la composition du produit sorti de ce même laboratoire. Ce produit va avoir, dès sa mise en circulation, une vie sans commune mesure avec les méthodes qui l'ont préalablement produit.

Le principe de symétrie incite Latour à traiter de la même manière les réussites et les échecs de la science, le rationnel et l'irrationnel; à refuser le "grand partage » qui a opposé jusqu'ici la culture à la nature, l'homme aux machines, la science à la religion (voir à ce propos du même Latour, Comment redistribuer «le grand partage», in «Revue de synthèse», 1983, n. 4, et dans la «Revue du M.A.U.S.S.», 1988, n. 1, pp. 27-65) et l'amène à «parler symétriquement de nous comme des autres sans croire ni à la raison ni à la croyance, tout en respectant à la fois les fétiches et les faits» (Petite réflexion sur le culte moderne des dieux faitiches, Le Plessis-Robinson, Synthélabo Groupe, 1996, pp. 9-10).

Cette ethnographie réflexive de la science débouche finalement sur une philosophie de la modernité. La société moderne est arrivée à ordonner la pratique (les savoirs pratiques) en mettant en place une série d'oppositions formelles (les savoirs théoriques): nature/société, objet/sujet, moderne/prémoderne, permanence/changement, transcendance/immanence, local/global, unité/diversité, égalité/inégalité, continu/discret, centré/a-centré, dépendance/indépendance, etc.

Ces oppositions ne sont que des asymétries, selon Latour. La modernité en fabrique à tour de bras, elle ne cesse de produire aussi de la dénonciation et de la critique de ces mêmes oppositions.

Brièvement dit, la modernité produit en théorie de l'asymétrie tandis qu'en pratique elle brasse, mélange, complexifie, assemble, métisse des êtres et des choses qui n'appartiennent pas au modèle théorique à prétention universaliste. La pollution, les embryons, le virus du SIDA, les robots à capteurs, l'informatique et tant de machines hybrides dominent notre vie quotidienne et pourtant nous continuons à privilégier comme étant uniques et exclusives les activités des êtres humains.

Pour toutes ces raisons, proclame Latour, il nous faut renoncer à être modernes : «Nous n'avons guère le choix. Si nous ne changeons pas la maison commune, nous n'y absorberons pas les autres cultures que nous ne pouvons plus dominer et nous serons à jamais incapables d'y accueillir cet environnement que nous ne pouvons plus maîtriser. Ni la nature ni les Autres ne deviendront modernes ».

Latour dit, certes, des choses importantes et intéressantes, mais sa tendance naturelle à tout radicaliser à outrance me les rendent suspectes.

Il ne fait aucun doute que l'entrée du sociologue dans le laboratoire est un événement important et que la description de ce qu'on y fabrique est passionnante, mais pourquoi tout réduire à un seul dénominateur? Peut-on assimiler, comme le fait Latour, la distribution des compétences entre la science et la politique à la controverse entre Thomas Hobbes et Robert Boyle? (Voir en la matière le très beau livre de Steven Shapin et Simon Schaffer, Léviathan et la pompe à air. Hobbes et Boyle entre science et politique, Paris, La Découverte, 1994). 
Si les identités et les différences sont produites et construites par des acteurs insérés dans des réseaux, pourquoi Latour accepte-t-il des objets déjà découpés tels, par exemple, que «science» et «technique», tels que «leptospire » et «spirochète», etc.? Si la construction d'un énoncé se fait dans un réseau et moyennant des réseaux, à quoi bon utiliser les intercitations pour reconstituer les réseaux scientifiques? Si le succès d'un énoncé doit être vu comme une construction élaborée par un réseau, comme un succès local, explicable par un rapport de forces particulier et momentané, comment expliquer que ce même énoncé devienne, à long terme, partie intégrante du noyau dur de la science? Comment se fait-il que certains concepts soient disponibles dans des conjonctures diverses et, en fait, discontinues? Peut-on nier l'existence d'un rapport instrumental d'une redoutable efficacité entre l'homme et le réel? Et que dire du «grand partage»?

Alors que la philosophie moderne a toujours hésité entre la suprématie du local (Kant) et du global (Hegel), dans les sciences, par contre, s'est vite affirmée la volonté systématique de subordonner le global à un impérialisme du local privilégiant l'action. Cette réduction méthodologique du global au local typique a produit, certes, des résultats significatifs dans nos savoirs, mais son transfert au monde de la vie quotidienne a suscité des problèmes inextricables.

C'est peut-être précisément pour ces raisons qu'on se plaît aujourd'hui à parler de «la fin de l'histoire» (F. Fukuyama), de «la fin de la nature» (B. McKibben), de «la fin de la politique» (M. Jacques), et ainsi de suite.

La mondialisation de l'information et des communications, l'économie planétaire, les processus de délocalisation des outils de travail et des hommes nous obligent à adhérer à une multiplicité de visions. Tiraillés entre les exigences de la mondialisation, qui donne une place importante au marché planétaire, et les conditions de la solidarité sociale et de la vie communautaire, les anciens sentiments d'appartenances sont en train de se vider de leurs contenus concrets tout en demeurant les derniers remparts pour sauvegarder nos identités collectives.

Je crois que les livres de Bruno Latour sont le reflet de cette crise, la manifestation sectorielle de sa perception; ils en constituent une poignante description.

Certes, Latour a de bonnes raisons de critiquer la distinction entre les «internalistes », soucieux uniquement d'analyser les énoncés scientifiques du point de vue épistémologique, et les «externalistes », préoccupés de décrire avant tout les lieux de production de ces mêmes énoncés. Latour a mille fois raison de répéter qu'il n'y a pas de solution de continuité entre «internalistes» et «externalistes », qu'il y a, bien au contraire, des liens étroits entre les hommes, les machines et la nature, et que c'est l'ensemble de ces connexions qui modélisent la science ellemême.

Une telle vision permet à Latour de nous offrir une analyse remarquable des logiques spécifiques qui gouvernent les pratiques expérimentales, une description lumineuse du mode de certification et des technologies de la preuve, une interprétation très fine des formes textuelles et matérielles de la transmission des savoirs, ainsi que des remarques intelligentes et perspicaces à propos de la conception de la pratique scientifique et des modalités d'exercice du pouvoir dans la science et par la science. Cependant, son option doctrinale de n'accorder aucune pertinence au contenu cognitif des énoncés scientifiques et de privilégier exclusivement le jeu social des actions et des activités scientifiques me semble plus que douteuse. 
Je crois qu'il vaut mieux comprendre pourquoi un énoncé, construit en un moment particulier et dépendant d'instruments spécifiques, est plus efficace qu'un autre pour rendre intelligible la connaissance de la réalité.

Le temps à ma disposition ne me permet pas d'analyser plus longuement les travaux de Latour et de les relier à ce courant intellectuel de notre époque qu'est le post-modernisme. Pour aller plus loin sur ce point, il faut lire: Michel Serres, Eclaircissements. Cinq entretiens avec Bruno Latour, Paris, Bourin, 1992; Francis Chateauraynaud, Forces et faiblesses de la nouvelle anthropologie des sciences, in «Critique», juin-juillet 1991, nn. 529-530, pp. 459-478 et Pierre Bourdieu, Raisons pratiques. Sur la théorie de l'action, Paris, du Seuil, 1994, pp. 91-97. Pour une lecture «critique» de la «Actant Network School», il faut consulter Jed Z. Buchwald, ed., Scientific Practice: Theories and Stories of Doing Physics, Chicago, Ill., University Press, 1995; A. Pickering, ed., Science as Practice and Culture, Chicago, University Press, 1992, ainsi que les articles de S. Schaffer, The Eighteen Brumaire of Bruno Latour, in «Stud. His. Phi. Sci.», vol. 22, n. 1, pp. 174-192, et O. Amsterdamska, Surely You are Joking, Monsieur Latour, in «Science, Technology and Human Values», vol. 15, n. 4, 1990, pp. 495-504.

Bruno Latour a présenté, d'une façon très brillante, lapidaire et simple, ses conceptions sur le capitalisme scientifique, sur le dynamisme de la recherche, sur les réseaux socio-technique, sur les rapports entre la science et la politique, entre l'humain et le non-humain, dans un texte adressé à un large publique, intitulé Le métier de chercheur. Regard d' un anthropologue (Paris, INRA Editions, 1995) et qu'il est profitable de lire attentivement.

\section{LE «PROGRAMME FORT» ET LES FONDEMENTS DE LA SCIENCE}

Le «programme fort» s'interroge aussi sur les fondements de la valeur de la science. Passons rapidement en revue les arguments majeurs que les tenants de ce courant intellectuel offrent à notre réflexion.

$\mathrm{Si}$ les règles méthodologiques auxquelles se rapportent les critères de démarcation entre la science et la non-science sont des conventions, si ces conventions peuvent être décrites comme les règles du jeu de la science empirique, selon la formule bien connue de Popper, il est alors inévitable de se demander quel est le fondement de ces conventions et de quelle source elles tirent leurs capacités persuasives.

Barnes répond ainsi à cette question: le fondement de la science se trouve dans la science elle-même conçue comme la valeur fondatrice. Les conventions scientifiques trouvent leur fondement dans un ordre institutionnel. Dès lors, ces conventions sont totalement semblables à celles de la religion ou à d'autres systèmes de croyance. Pour Barnes et Bloor, il est vraiment impossible de fonder la supériorité de la science sur tous les autres systèmes symboliques. Tous les arguments soutenant la primauté de la science sont fondés sur des préjugés favorables à la science, sur un type de développement culturel propre à l'Occident.

Que la science soit fondée sur une valeur (la croyance selon laquelle il est possible, grâce à certaines procédures, de dévoiler le caché et la croyance en l'efficacité et en l'efficience des méthodes d'investigation scientifiques) est incontestable. 
Depuis le constructivisme kantien en gnoséologie et surtout depuis que Max Weber nous a dévoilé les processus de rationalisation, nous savons qu'à l'origine de la science moderne, il y a des choix de valeurs. Mais nous savons également que la solution des problèmes, même des problèmes engendrés par le progrès techno-scientifique, ne peut être fournie que par la science elle-même.

Faut-il citer à titre d'exemple l'éradication de certaines maladies ou encore l'informatique et la robotisation?

Admettons qu'au départ il y ait une valeur, et que cette valeur soit intériorisée par les savants. Peut-on négliger le fait que cette valeur fondatrice affirme que les concepts et les théories scientifiques sont déterminés de manière univoque par une réalité objective, atteignable d'une manière ou d'une autre, et ceci indépendamment de ce que le savant souhaite ou espère?

Weber, qui a écrit des pages admirables à ce propos, affirme que la science est une entreprise digne d'être poursuivie car elle est dotée d'une valeur notable, même si cette valeur ne peut pas être toujours «scientifiquement» démontrée.

De ce point de vue, les conventions scientifiques ne trouvent leur justification que dans la perspective d'une connaissance intersubjectivement contrôlable. Or ce statut de connaissance est possible parce que les savants agissent selon une méthode orientée par la valeur du contrôlable et de l'intersubjectivement vérifiable. C'est exactement la «Wertfreiheit» de Max Weber.

Pour les tenants du «programme fort », le caractère conventionnel des critères qui permettent le choix des procédures et des méthodes a un sens foncièrement différent de celui de Max Weber, de Karl Popper, et en général des rationalistes critiques.

Tout d'abord, Bloor et Barnes considèrent que les procédures et les méthodes des sciences sont des conventions. Il en va de même de toutes les théories mais également des résultats de la recherche scientifique. Bloor et Barnes admettent que ces conventions ne sont pas arbitraires, qu'elles doivent être crédibles socialement et utiles pratiquement. Cependant, elles ne peuvent être ni auto-évidentes, ni universelles, ni statiques. Effectivement, elles doivent être acceptées par un groupe social, et celui-ci ne les acceptera que si elles ne sont pas en contradiction avec ses modèles normatifs et pour autant qu'elles soient efficaces et efficientes.

Mais peut-on dire qu'une théorie acceptée est une théorie vraie? Peut-on réduire la vérité à une acceptation?

Bien sûr que non, répond Bloor, et pourtant l'acceptation intègre la théorie dans le système cognitif du groupe et lui donne ainsi les moyens de comprendre le monde. Certes, la théorie scientifique prévoit que la critique radicale d'un système de connaissances propre à un groupe social donné demeure toujours possible, sous certaines conditions. Mais il est également vrai, ajoute Bloor, que cette critique n'est possible que dans la mesure où il existe des raisons de l'exploiter. Or cette possibilité, dans nos sociétés bureaucratisées, est hautement improbable, voire impossible.

L'argumentation de Bloor ne me paraît pas convaincante. Si les critères méthodologiques sont des conventions, les procédures et les théories acceptées sur la base de ces critères en sont également.

Etant donné que ces critères, ces procédures, ces théories ne peuvent pas être vrais, la seule chose dont nous puissions être sûrs est celle-ci: ils sont crédibles pour le groupe social qui les accepte et qui les utilise pour s'orienter. La crédibilité devient ainsi une affaire politique et il en va de même de la possibilité de critiquer radicalement une théorie. 
Le «programme fort» ne sait pas ou ne veut pas distinguer la crédibilité de la validité. Il confond les deux notions: validité et crédibilité pour soi et pour le groupe deviennent la même chose. Il y a là une confusion fâcheuse. La validité de certaines connaissances, décidée sur la base de certaines procédures et méthodes, après vérifications et critiques, ne peut pas être confondue avec un consensus social élaboré dans un contexte déterminé.

Bien sûr, la science peut s'autodécrire comme si elle était un jeu, mais contrairement à ce que l'on peut observer dans d'autres univers symboliques, la science procède volontairement à partir de règles fixées préalablement, moyennant certaines procédures élaborées de façon rationnelle. Pour cette raison, la science ne peut pas être dogmatique à propos de toutes les règles. Elles sont respectées pour autant qu'elles fonctionnent; elles sont rejetées si elles ne fonctionnent plus ou fonctionnent mal. La science est antidogmatique parce qu'elle est capable de tout mettre en discussion et de tout abandonner, même ses critères les plus éprouvés et primordiaux.

Les tenants du «programme fort» l'oublient trop facilement, et cela rend la discussion avec eux assez difficile. En effet, ils ont trop tendance à réduire la science et son analyse critique à cette certitude infondée qui, d'après Ludwig J. Wittgenstein (1889-1951), caractérise le jeu linguistique de la vie quotidienne. À sa base, il y aurait moins une proposition infondée qu'une façon d'agir, un mode d'agir infondé.

Poussés par l'ambition d'étudier la science de façon naturaliste, les tenants du «programme fort» finissent par la réduire à une sorte de phénomène congénial à la société moderne et totalement dépendant de celle-ci, tant dans ses règles que dans ses contenus.

Les tenants du «programme fort», convaincus que la démarcation entre science et non-science est une affaire de critères analytiques acceptés par convention, considèrent l'acceptation et le refus comme des actes politiques. La science est alors réduite à ce que les institutions sociales définissent en tant que telle. Suffit-il d'être à la tête d'une institution sociale pour produire de la science? Certainement pas. La science vit et se déploie dans des institutions, mais l'agir scientifique n'est pas réductible à la logique de ces mêmes institutions. Il les dépasse, il les contourne, il a une logique propre qu'il faut comprendre pleinement avant de la contester ou de la nier.

Après les postulats de l'autofondation logico-formelle de la science, de la démarcation science/non science et du fondement/valeur de la science, le quatrième postulat du «programme fort » énonce l'impossibilité d'élaborer un critère suffisamment puissant pour choisir rigoureusement entre des théories rivales. A ce propos, Jean Piaget a rédigé, dans le volume de l'«Encyclopédie de la Pléiade» intitulé Logique et connaissance scientifique (Paris, Gallimard, 1967) ainsi que dans ses Etudes sociologiques (Genève, Droz, 1977), des pages claires et lumineuses que je vous conseille de lire très attentivement afin de bien saisir les enjeux d'une question très compliquée et que le réductionnisme du «programme fort» formalise à l'excès.

Le «programme fort» fait un usage étendu des principaux acquis théoriques de l'ethnométhodologie, notamment de deux de ses options: (a) tous les objets sociaux sont construits moyennant diverses démarches collectives; (b) dans la vie sociale, il y a plusieurs types de rationalité à l'œuvre, qui sont fonction de diverses choses et circonstances. 
Le «programme fort» adopte ces options intellectuelles surtout dans le but de désacraliser la science, de montrer que la science s'explique complètement et sans résidus lorsqu'on parvient à la ramener aux facteurs sociaux qui la déterminent.

Pour le «programme fort», tous les aspects de l'activité scientifique, concepts et théories compris, sont toujours tributaires d'une structure sociale donnée.

Le «programme fort» croit démontrer l'existence d'un lien solide sociétéscience grâce à une reconstruction ponctuelle et minutieuse de telle ou telle recherche, de telle ou telle élaboration théorique. Toutefois, comme l'a déjà noté F.-A. Isambert dans un article remarquable et que j'ai déjà cité plusieurs fois, ces recherches visent, outre la reconstruction du déroulement singulier de l'épisode ou de l'événement, à «passer de l' 'expliquer comment' à l' 'expliquer pourquoi', et là un saut est inévitable. On fait appel à une conception plus générale des idéologies, des motivations des chercheurs ou même de l'objectivité scientifique. Ce qui est grave, c'est quand ce saut n'est pas perçu, quand on tombe dans l'illusion, bien connue des historiens, qui consiste à croire que l'on a détecté les causes parce qu'on a raconté la succession des faits par le menu (aura-t-on une 'petite sociologie' comme il y a une 'petite histoire'?) ou encore qu' on a rendu caduques certaines explications parce qu'on les a remplacées par un récit. On est ainsi renvoyé à l'attribution d'un rôle explicatif global à 'la société', 'les facteurs sociaux', etc. Or ce qui est frappant, dans l'ensemble de ces analyses, c'est l'incapacité où elles sont d'assigner un rôle explicatif précis et cohérent à cette société qu'elles invoquent.» (F.-A. Isambert, art. cit., p. 503).

Au fond, derrière le programme de recherche qui se propose de démontrer que les activités scientifiques n'ont pas d'autonomie dans l'ensemble des activités intersubjectives qui constituent la société, Isambert aperçoit, et je crois avec raison, une ambition métaphysique: expliquer la nature même de la science. Ce projet, malgré son sociologisme apparent, serait-il davantage philosophique et anthropologique que sociologique?

Je le crois. Le «programme fort» opère une véritable clôture circulaire, de la science à la société et de la société à la science. Ainsi la science est vidée de toute spécificité par rapport à toutes les autres formes de connaissances et de croyances.

La validité des connaissances scientifiques n'a, en conséquence, d'autre statut que celui que lui confère la crédibilité sociale d'un contexte historique ou socioculturel donné.

Le «programme fort» nous propose une explication causale et déterministe des activités scientifiques, explication considérée comme plus performante que l'explication téléologique (système de rapports entre moyens et fins ou rapports de finalités).

Cette explication causale ramènerait les croyances des agents sociaux aux critères de validité propres aux interprètes, et respecterait aussi le principe fondamental de la symétrie. Je crois, pour ma part, que cette explication causale est incompatible avec une théorie scientifique basant sa crédibilité sur des critères de validité logico-analytiques partagés par une communauté scientifique. Le principe de symétrie ne peut pas s'appliquer à toutes les croyances, à une croyance fondée sur la crédibilité sociale, et à une autre fondée sur la validité établie à partir de conventions librement acceptées par les chercheurs. 
Si les tenants du «programme fort» veulent fournir une explication causale de ces connaissances, ils doivent alors nécessairement faire appel à une crédibilité autre que la leur et à des croyances qui, pour eux, ne sont pas crédibles. Dans ce cas, la symétrie entre l'analysé et l'analyste disparaît.

Explication causale et symétrie vont rarement ensemble dans l'enquête sociologique.

Reste à dire quelques mots sur le principe de réflexivité. D'après ce principe, la sociologie de la connaissance est susceptible, elle aussi, d'explication sociologique. Mais si l'acteur social et l'interprète partagent les mêmes critères de validité au sujet d'une croyance, comment faire pour éviter que la réflexivité ne soit pas une simple paraphrase, une glose interlinéaire, voire une glossolalie?

Le programme fort réduit le concept de validité au concept de crédibilité sociale. Or la science fait une distinction foncière entre ces deux concepts, mais aussi entre la validité fondée sur des règles et la crédibilité des règles. Elle nous indique aussi la différence existant entre l'agir orienté de manière cognitive et l'agir social orienté par la passion, les sentiments, les instincts, bref par le nonlogique.

Le programme fort est dans l'impossibilité de résoudre convenablement tous ces problèmes. Certes, il a attiré notre attention sur une série de questions fondamentales, primordiales. A-t-il pour autant déstabilisé les programmes de recherche de la sociologie mertonienne et post-mertonienne? A-t-il dit quelque chose de tellement nouveau, au point que la tradition sociologique en soit ébranlée?

Je ne le crois pas. Il a montré le point faible de la sociologie de la science contemporaine mais il n'a pas été capable, jusqu'ici, de lui opposer un contreprojet cohérent et plus solide.

\section{LES THÈSES PRINCIPALES DU «PROGRAMME FORT»}

Contrairement aux sociologues tenants du «programme fort», les sociologues «classiques», de Durkheim à Merton, ont été unanimes à définir la science comme un phénomène échappant, tout au moins dans son noyau dur, à l'analyse sociologique. Ils ont été pleinement conscients de la place capitale que la science occupe dans les sociétés modernes, du rôle exceptionnel qu'elle y assume. Ils ont toujours été de l'avis que les méthodes scientifiques nous dévoilent les mystères de la matière et nous préservent des risques de l'égocentrisme et du sociocentrisme.

Pour ces mêmes sociologues «classiques», il est notoire que les méthodes scientifiques ont été élaborées dans des organisations composées de savants, selon des règles conventionnelles préétablies par eux, et dont la finalité principale consiste à préserver le travail scientifique des déformations produites par des facteurs existentiels et/ou sociaux, à le soustraire aux démarches et aux interprétations arbitraires, au libre choix des principes et des conventions.

Merton est le sociologue le plus représentatif de cette façon de concevoir la sociologie de la connaissance scientifique. Il n'a jamais contesté la thèse selon laquelle les sciences restent redevables de l'époque du savant, du contexte sociohistorique et de l'espace social qu'il occupait. Cependant, Merton a toujours 
refusé d'établir une corrélation directe entre la connaissance produite par le savant et ses positions et conditions dans le temps et dans l'espace social.

Pour lui et ses disciples, la sociologie ne peut rien dire de valable sur la forme et le contenu de la connaissance scientifique. Le monde physique et le monde social demeurent distincts. Pour cette raison, la science est une construction différente de toutes les autres constructions ou phénomènes sociaux.

Connaissance du réel, connaissance certaine, indubitable, démontrable, selon la formule de K. R. Popper (The Myth of the Framework. In Defence of the Science and the Rationality, London, Routledge, 1995), la science est prise en charge par la sociologie lorsqu'il est nécessaire de révéler les implications sociales de la connaissance scientifique, ou encore lorsqu'il faut mettre en évidence les difficultés ou les avantages de l'exercice de l'activité scientifique dans un système social donné, ou les conséquences sociales de cette même activité pour un groupe déterminé.

Pour quelles raisons les sociologues «classiques » limitent-ils les compétences de la sociologie, alors que les sociologues tenants du «programme fort» les accroissent-ils considérablement?

Nul doute que les sociologues «classiques», à l'exception peut-être de Sorokin, sont tous persuadés que la Science est universelle parce que la nature est stable, permanente et uniforme.

Contrairement au monde social-historique, le monde naturel serait doté, selon ces sociologues, d'objets et de procès invariants, stables, d'où le constat que certains événements s'y déroulent de façon cohérente et que les relations observables y sont invariables. La stabilité des faits prouverait l'universalité des lois physiques. Entre la théorie et les faits observables, il y aurait donc un rapport spécifique. Les faits seraient toujours neutres. Ils représenteraient la réalité physique observée, ils ne seraient pas modifiables, en leur contenu et en leur signification, par les interprétations des uns et des autres. En réalité, il reviendrait aux faits de discriminer, en dernière instance, entre les théories vraies et les théories fausses. Jamais une théorie ne peut rendre compte de tous les éléments dont un fait est constitué. Les faits existent en dehors de la théorie qui les a individualisés. Une théorie peut se périmer, mais le fait subsistera malgré tout, indépendamment de la théorie qui l'a identifié.

Analysons brièvement cette conception de la science en tant que connaissance exacte, universelle et vérifiable exprimée par des lois.

Tout d'abord qu'est-ce qu'une loi universelle?

Les lois universelles sont des vérités concernant la nature, basées sur des énoncés factuels, établis sur la base d'observations minutieuses, précises, attentives, répétées. L'accumulation de ces observations accroît notre connaissance du monde physique et permet une généralisation toujours plus grande de nos énoncés sur ce même monde. La science progresse par accumulations et généralisations successives. Elle est déterminée et exclusivement garantie par le monde physique, invariant, stable et uniforme.

Mais de quelle façon les sociologues «classiques» conçoivent-ils l'invariance, la stabilité et l'uniformité du monde physique, en somme l'universalité du monde naturel?

En la matière, nous ne disposons que des travaux de N.R. Hanson (Patterns of Discovery, Cambridge, Cambridge University Press, 1965; Perception and Discovery, San Francisco, Freeman \& Cooper, 1969; What I do not believe, and 
others essays, Dordrecht, D. Reidel, 1971; Observation and explanation : a guide to philosophy of science, London, G. Allen \& Unwin, 1972; Constellations and conjectures, Dordrecht, Boston, D. Reidel, 1973). Grâce à ces travaux, nous connaissons les usages qu'on fait régulièrement du principe d'uniformité de la nature et nous savons comment les sociologues sont parvenus à la conviction suivant laquelle ce principe d'uniformité est vrai et certain.

Hanson montre que la vérité du principe d'uniformité a été fabriquée tantôt grâce au formalisme (les vérités scientifiques reposent sur des conventions ou des définitions de symboles, ce qui compte c'est l'enchaînement des énoncés, par contre leurs contenus et leurs significations sont négligés), tantôt grâce à l'empirisme qui proclame que nos connaissances sont des produits de l'expérience, qu'elles naissent de l'expérience, de la nature, jamais de la raison et de l'esprit.

Mais comment certifier sans ambages que le formalisme et l'empirisme, avec leur complément, l'expérimentation, sont en mesure de nous fournir des données concrètes, perceptibles par les sens, sur le monde matériel?

Aucun de nous ne peut garantir une telle affirmation.

Si l'uniformité de la nature était un principe vrai, alors il devrait préexister aux procédures empiriques qui le façonnent. Les données produites par l'expérience ne permettent pas de généraliser une validité obtenue ponctuellement, de manière sectorielle, ici et maintenant. Il est impossible d'inférer la généralisation d'une telle validité sur la base d'observations particulières. Pour ce faire, nous devons présupposer que toutes les expériences et toutes les observations puissent être, d'une manière ou d'une autre, validées. Ce que nous faisons lorsque nous affirmons, par exemple, que probablement la vie existe seulement sur la Planète Terre, que les lois de la gravitation sont universelles, que l'univers est fini ou infini, ou encore que toutes les mouches sont noires et que tous les cygnes sont blancs.

Puisque il a été jusqu'ici impossible de donner au principe d'uniformité de la nature les caractéristiques d'un énoncé général, ni même de prouver qu'il est la marque spécifique du monde physique, les sociologues, selon Hanson, se sont fourvoyés en lui reconnaissant une validité, par ailleurs très douteuse et indéfinissable. De plus, ils ne se sont pas aperçus que ce principe est le produit des méthodes d'investigations utilisées, le fruit d'un processus enclenché par le savant en vue d'obtenir l'explication du phénomène. Par conséquent, si les sciences naturelles élaborent des représentations valides, leur validité, c'est-à-dire la conformité d'un élément réel avec sa représentation, dépend des circonstances d'espace et de temps propres au chercheur.

En outre, Hanson rappelle qu'une théorie est une construction intellectuelle rattachant des faits ou des lois à un principe à partir duquel ils peuvent tous être déduits. Une théorie aide à faire des observations et reste soumise au contrôle de l'expérience. C'est aux observations de valider la théorie. Il y a un rapport solide entre ces deux moments, bien qu'il s'agisse de deux moments distincts.

Mais si l'observation dépend de la théorie, une modification de la théorie doit nécessairement provoquer un changement dans l'observation. Ainsi, la révision des preuves empiriques est inévitable. Dans ces conditions, la circularité devient flagrante: mon raisonnement postule les lois universelles du monde physique, mais ces dernières ne sont validées que par les expériences fabriquées par ma propre théorie et la validation de ma théorie est réalisée par ces mêmes expériences. 
Peut-on sortir de ce cercle vicieux?

Popper a cru en sortir en affirmant qu'il est impossible de prouver la vérité des théories scientifiques, dont la nature est hypothétique. Tout au plus, nous pouvons les falsifier. Mais si nous affirmons que les énoncés d'observation sont constitués grâce à des théories hypothétiques, nous devons alors admettre qu'ils sont soumis à des révisions constantes. S'il en est ainsi, la stabilité, l'uniformité et la permanence des faits - fondements de l'épistémologie qui attribuent à la science une singularité irréductible - en sont fortement et irrémédiablement fragilisés.

Une telle conception présuppose que la connaissance scientifique est composée de faits et de théories, d'objets observés avec des contenus empiriques observables et d'objets non observables ou théoriques. Une telle conception postule également qu'entre les propositions factuelles et les propositions théoriques, entre les objets observables et les objets non observables, il doit y avoir, par une conséquence inévitable, un rapport d'interdépendance.

Si l'on admet que les propositions factuelles sont des relations entre des objets observables et que ces relations sont établies grâce à des objets non observables, a-t-on pour autant démontré la nature fallacieuse du projet cognitif de la science? Le contenu cognitif de l'activité scientifique risque-t-il d'être dilapidé si l'on ajoute que toutes les constructions théoriques sont des fictions, qu'elles ne peuvent être établies, si non indirectement et de façon spéculative?

Pour donner des réponses plausibles à ces questions, il faudra tout d'abord nous interroger brièvement sur la question des rapports entre la théorie et la pratique.

\section{LES RAPPORTS ENTRE LA THÉORIE ET LA PRATIQUE}

Opposée à la pratique (ce qui se fait, l'expérience que l'on a, un savoir-faire), la théorie est une construction intellectuelle (concepts définis, objets construits, relations vérifiables, enchaînements déductifs, langage contrôlable) grâce à laquelle des faits ou des lois sont méthodiquement rattachés à un principe d'où, ensuite, ils doivent être logiquement déduits.

Les rapports entre la théorie et la pratique ont suscité des discussions interminables. En simplifiant à l'excès, on peut dire que les quatre points de vue les plus courants sont: (1) le conceptualisme (la connaissance relie les concepts par le prédicat et cherche l'accord de la pensée avec la réalité); (2) le nominalisme (primauté du nom des choses, des valeurs syntaxiques et sémantiques des signes dans le langage); (3) le réalisme (primauté de la réalité, dont la connaissance dépend); (4) l'idéalisme (la réalité est réduite aux représentations, les objets n'existent ni en nous ni hors de nous, ni même comme des choses en soi, mais seulement comme des représentations réglées par des principes).

Ces quatre points de vue conditionnent toutes les approches entre la théorie et la pratique et donnent lieu à une multitude d'affirmations: la théorie cherche l'accord avec la réalité; la théorie précède la réalité; la réalité précède la théorie; la réalité n'est pas indépendante de la connaissance que nous en avons.

Il m'est impossible d'analyser dans un séminaire de sociologie tous ces points de vue, d'autant plus qu'ils relèvent davantage du débat philosophique que de 
celui des sciences sociales. Je me bornerai à discuter ici l'affirmation la plus courante parmi les scientifiques, celle que Jean Ullmo (La pensée scientifique, Paris, Flammarion, 1969) a très bien résumée ainsi: «... la science recherche ses objets, elle les construit, elle les élabore; elle ne les trouve pas 'tout faits', tous donnés dans la perception ou l'expérience immédiate. Le monde de la science est une construction; les méthodes de cette construction, constituent la première étape de la science...».

En somme, la théorie permet de donner une consistance, de voir les faits, mais ces derniers existeraient indépendamment d'elle; voire: ils continueraient à exister, même si la théorie qui a permis leur individualisation était dépassée par une théorie plus générale.

Sans pour autant trancher nettement ces questions, disons que pour une très grande majorité de savants (les sociologues sont moins péremptoires), il faut distinguer, dans la connaissance scientifique, deux niveaux: les faits et les théories, les objets empiriques aux contenus observables et les objets théoriques aux contenus non observables. Les rapports synallagmatiques entre la théorie et le fait, par contre, sont plus compliqués et par ailleurs ils varient d'un courant intellectuel à l'autre.

En vérité, cette distinction entre les faits et les théories me semble assez problématique, pour ne pas dire très énigmatique, bien que la thèse extrême («En théorie, les théories existent, en pratique elles n'existent pas.») défendue par Bruno Latour (Sur la pratique des théoriciens, in Savoirs théoriques et savoirs d'actions. Publié sous la direction de Jean-Marie Barbier, Paris, PUF, 1996, pp. 131-146) appelle de ma part beaucoup de réserves et réticences.

Dans la physique des hautes énergies, l'anti-matière, par exemple, est une construction hypothétique dérivée d'expériences faites avec des objets tout à fait réels et observés grâce à des appareillages construits pour cet effet.

Le physicien britannique Paul Dirac (1902-1984) est l'un des créateurs de la mécanique quantique. Prix Nobel en 1933, il a élaboré, en 1927, une théorie de l'électron qui tient compte simultanément des théories de Erwin Schrödinger (1887-1961) et de Werner Karl Heisenberg (1901-1976), de la théorie de la relativité et du spin (moment cinétique intrinsèque d'une particule). Grâce à cette théorie, il a déduit l'existence de l'anti-électron (positon) et donc de l'antimatière. En 1932, Carl David Anderson (1905-1991) découvrit que le positon, c'est-à-dire les anti-électrons se trouvent effectivement dans les rayons cosmiques. Il donnait ainsi la première confirmation expérimentale de la prédiction formulée par la théorie de Dirac. Mais c'est en 1981 que les chercheurs du CERN sont arrivés à produire ces antiparticules éphémères et à les faire tourner en paquets serrés dans de grands anneaux vides. Il faudra attendre 1985 pour avoir la preuve expérimentale ultime de la théorie de Dirac grâce à l'allemand Walter Oelert qui est parvenu, toujours dans les laboratoires genevois du CERN, à créer neuf atomes d'anti-matière, dont la durée de vie n'a guère dépassé les quarante milliardièmes de secondes!

Afin d'observer ces neuf atomes d'anti-hydrogène, les chercheurs du CERN ont dû construire des appareils, les accélérateurs, qui permettent d'observer l'antimatière. Bien évidemment, cette observation se fait grâce à des enregistrements effectués par d'autres machines mises au point expressément pour les recherches sur l'anti-matière. 
Le cas de la production de la première paire de particules fondamentales appelées W+ et W-, portant la quantité de charge d'un électron (le premier positif et le second négatif), est très emblématique. Ces bosons intermédiaires, en quelque sorte forces faibles régnant à l'intérieur de la matière et permettant la cohésion des particules à l'intérieur de neutrons, avaient été prédits par le modèle standard de la matière mais jamais observés empiriquement. Ces particules ont été observées récemment grâce aux modifications apportées au LEP, le super-collisionneur du CERN capable de lancer les particules à une vitesse proche de la lumière dans un anneau souterrain de $27 \mathrm{~km}$.

Le modèle standard de la matière prévoit l'existence du boson dit de Higgs, mais personne jusqu'ici n'est arrivé à le saisir. Si un collisionneur plus puissant parvient un jour à le mettre en évidence, la théorie, une fois de plus, aura précédé la réalité.

Ces exemples montrent combien les rapports entre la théorie et la pratique sont complexes et compliqués. Nous constatons qu'il est risqué d'accorder un primat aux faits sur la théorie ou d'affirmer que la théorie est vérifiée par les faits.

Vous avez vu que la théorie de Dirac accorde la primauté aux noms des choses, aux valeurs syntaxiques et sémantiques des signes dans le langage formel, avant que d'en chercher la correspondance avec les choses réelles. Dirac a spéculé sur l'anti-matière, il a construit une théorie axiomatique, il a élaboré une doctrine cohérente sur le plan logique, mais sa théorie n'a connu sa vérification ultime, par les faits, que cinquante ans plus tard environ. Et pourtant cette théorie a été considérée comme vraie dès son élaboration. Pourquoi? Est-ce dû à l'excellente réputation de théoricien de Dirac? Faut-il l'attribuer à la démonstration magistrale qu'il était parvenu à donner de l'équivalence des deux formulations de la théorie quantique, la matricielle de Heisenberg et l'ondulatoire de Schrödinger?

Je ne suis pas en mesure de répondre à cette question. Cependant le fait est là. L'équation de Dirac fut immédiatement considérée comme une expression bien formée, une théorie originale et fondée, bien que non vérifiée empiriquement.

Imaginons maintenant que j'aie de bonnes raisons de nier l'existence de l'antimatière, de bonnes raisons de contester l'équation de Dirac, il n'en reste pas moins que je ne peux pas mettre en doute le fait qu'il y a, au CERN, des machines qui ont révélé l'anti-matière. Certes, la théorie de Dirac est une construction hypothétique, je peux la critiquer et la réfuter aux moyens des langages formels avec lesquels elle a été construite, mais mes arguments, mes critiques, mes objections, sont inappropriés à l'endroit des appareillages qui ont révélé l'existence de l'antimatière, qui ont confirmé les prédictions du physicien anglais.

Cet exemple montre bien les difficultés que nous avons à distinguer les objets observables des objets non observables, les objets observés directement des objets construits par inférence à partir de caractéristiques de certains effets.

Lorsque j'observe la chute d'un corps dans l'espace, le rapport de la théorie au fait ne soulève aucune difficulté. Mais lorsque l'observation n'est possible que grâce à des machines, à des appareillages, à des sensitomètres, à des détecteurs et ainsi de suite, comment puis-je séparer le fait de la théorie? Comment puis-je parler d'un fait indépendamment de la théorie, si c'est bien celle-ci qui m'en a permis l'identification? De quelle façon parler dans ce cas d'une différence fondamentale entre les énoncés théoriques et les énoncés d'observation? Sur quelles bases affirmer que les énoncés théoriques sont problématiques, tandis que les énoncés 
d'observation sont neutres? Dans cette dernière perspective, de quelle façon les énoncés d'observation arrivent-ils à établir la validité des énoncés théoriques?

On pourrait m'objecter que ces questions n'ont un sens que dans le cas où la distinction entre la théorie et le fait est encore possible. Mais peut-on négliger qu'un tel cas d'espèce est de moins en moins généralisable?

Il y a également des cas où la théorie n'est ni vraie ni fausse et où il est pratiquement impossible de dire qu'une telle théorie produira des connaissances valides. On peut seulement constater que la science fait un usage étendu de certains énoncés sans pour autant être en mesure de les valider empiriquement. Il en a été ainsi de la théorie de Dirac pendant environ cinquante ans; il en est ainsi aujourd'hui de tant d'autres théories.

Je reconnais, bien évidemment, que beaucoup de théories utilisées ne renvoient à aucun objet réel et que leurs significations peuvent être très différentes des énoncés d'observation. J'admets aussi que ces théories sont essentiellement des systèmes formels, des outils logiques permettant de déduire de nouveaux énoncés d'observation. Mais il faut reconnaître qu'avec de telles supputations, les risques de dévaloriser considérablement le travail théorique sont énormes, alors même que ce travail occupe une place prééminente dans toutes les activités de la cité scientifique et dans les pratiques journalières des savants.

En radicalisant la distinction entre théorie et pratique, ne risque-t-on pas de faire surgir des frontières dans les laboratoires et sur les terrains où elles n'existent pas? Ces frontières ne seront-elles pas posées par les savants, tantôt empiriquement tantôt spéculativement, ou pour des raisons tactiques ou stratégiques? Personne ne peut contester que beaucoup d'énoncés d'observation reçoivent un sens, dans le laboratoire et sur le terrain, essentiellement grâce à la place que leur accorde la théorie. Sans cela, ces énoncés seraient muets et aveugles, ils n'auraient aucune signification.

Si les énoncés d'observation ont une signification dans la mesure où la théorie leur donne une place dans un ensemble, alors les propositions factuelles dépendent de la théorie. Leur stabilité est une fonction de la théorie.

Une théorie peut demeurer stable longtemps et pourtant voir son sens évoluer en fonction du contexte interprétatif de la recherche. L'exemple classique nous est donné par la théorie immunitaire. La signification attribuée par cette théorie à certains énoncés factuels et l'interprétation qui en était donnée a changé d'une catégorie de chercheurs à l'autre, suivant que leurs recherches portaient sur l'immunochimie, sur les immunodépresseurs, sur l'immunocompétence, sur l'immuno-déficience, ou sur l'immunofluorescence, etc.

La signification d'un même énoncé varie aussi en fonction des interprétations qu'en donnent des chercheurs appartenant à des disciplines différentes, ou des enseignants s'adressant, par exemple, à des étudiants en épistémologie, à des étudiants en physique théorique, ou encore à des étudiants en sciences sociales.

Je suis convaincu que les faits scientifiques dépendent très fortement non seulement de la théorie, mais aussi des interprétations qu'on en donne dans certains contextes, des focalisations effectuées par les chercheurs, par l'accent placé sur tel ou tel aspect d'un phénomène ou encore par l'exercice de telle ou telle spécialisation.

Ceci dit, il faut ajouter qu'une théorie est aussi une construction langagière et que les propositions factuelles sont toujours nécessairement formulées dans un 
langage formel ou dans un langage naturel. Un fait existe si l'on a des ressources symboliques pour le représenter, des mots pour le décrire. Si ces ressources manquent, ou sont insuffisantes, alors la formulation des énoncés factuels est irréalisable. Jean-Blaise Grize a écrit à ce propos des pages lucides et perspicaces (Logique naturelle et communications, Paris, PUF, 1996) qu'il faut lire et relire.

De plus, N.R. Hanson a démontré de la manière la plus convaincante que lorsqu'on a recours à certaines notions de la théorie physique, on se trouve dans l'impossibilité absolue d'observer et de représenter une série d'aspects importants du monde matériel. C'est dire que les langages formels et naturels conditionnent profondément les théories et les faits, mais jamais de façon absolument univoque. Deux savants, utilisant le même langage, selon les mêmes procédures, peuvent formuler des hypothèses totalement contradictoires. Ils peuvent aussi démontrer logiquement une proposition et se trouver dans l'incapacité d'en donner les vérifications empiriques. La théorie de l'expansion de l'univers ou celle du trou noir, constructions théoriques par excellence, en sont des exemples significatifs parmi bien d'autres.

L'histoire des sciences est riche de cas de ce genre. Un seul rappel ici suffira à illustrer mon propos: le cas de Darwin qui produisit des hybrides en croisant les végétaux $\mathrm{A}$ et $\mathrm{B}$. L'observation lui montra qu'à la première génération il y a la dominance (dans 88 exemplaires) de certains caractères de l'espèce $\mathrm{A}$, tandis qu'à la deuxième génération il y a la présence (dans 37 exemplaires) des caractères des deux espèces croisées, A et B. Pourquoi ce rapport d'environ 1 à 3 ? Darwin ne sut pas l'expliquer.

L'explication sera donnée par Johann Gregor Mendel (1822-1884) grâce à une formulation «linguistique» plus précise. Il fit appel aux chromosomes et aux caractères héréditaires, moyennant quoi l'explication du rapport devint possible.

Maintenant nous pouvons commencer à conclure. La distinction entre fait et théorie soulève d'énormes réserves. Le fait existe en fonction d'une théorie. C'est toujours la théorie qui donne une signification au fait. Une telle signification change en fonction de la place attribuée par la théorie au fait. J'admets aussi que la signification d'une théorie puisse provenir de la métascience qui fonde la science. A ce propos, je ne peux que vous renvoyer à ce que j'ai déjà dit, notamment au le chap. IV de mes Questions actuelles de sociologie de la science (Lausanne, IASUL, 1995).

Ayant reconnu cela, dirais-je également que chaque théorie constitue un monde fermé et que les théories restent incommensurables et incomparables entre elles?

L'état de la question en la matière a été dressé par Evandro Agazzi (Commensurability, Incommensurability and Cumalativity in Scientific Knowledge, in «Erkenntnis », XXII, 1985, pp. 51-77). Je me bornerai à dire ici que ma réponse est simple: l'incommensurabilité et l'incomparabilité concernent la métascience, mais jamais la science. Il est impossible d'établir de mesures communes entre l'astrologie et l'astronomie, entre la parapsychologie et la psychologie, entre l'alchimie et la physique. Par contre, les théories scientifiques, fondées sur un même métalangage sont comparables et commensurables. L'exemple classique nous est donné par la théorie quantique matricielle et par la théorie quantique ondulatoire. Un autre exemple nous est donné par l'extension de la théorie de la relativité générale à la gravitation et par la théorie unitaire englobant aussi bien l'électromagnétisme que la gravitation. 
Les savants élaborent des théories en critiquant d'autres théories ou en les perfectionnant. Ils partent toujours d'une théorie existante, il l'analysent, l'améliorent ou la réfutent. Lorsqu'ils doivent choisir une théorie entre plusieurs, le choix est dicté par des critères de pertinence et de rigueur logique, mais aussi par des critères métascientifiques et des préconstruits culturels.

Depuis Kuhn et la nouvelle philosophie de la science, l'incommensurabilité des théories est à la mode. Mais il s'agit d'une dispute académique attirant très peu l'attention des savants. Vous trouverez un inventaire de ces discussions dans le livre de D. Oldroyd (The Arch of Knowledge. An Introductory Study of the History of the Philosophy and Methodology of Science, London-New York, Methuen, 1986).

Ma conclusion est simple: contrairement aux sociologues «classiques», je crois que la science se base sur une métascience et un métalangage qui conditionnent, d'une certaine manière, la science et ses théories. Il y a là, assurément, un domaine important de recherche pour le sociologue. Ceci dit, l'élaboration d'une théorie se fait à partir de normes et de conventions fixées sur la base de modèles normatifs plus ou moins épurés par une communauté de pairs. Une fois qu'il a été établi que ces modèles normatifs n'ont aucune corrélation directe avec les facteurs sociaux, l'analyse externaliste doit laisser la place à l'analyse internaliste. Cette dernière n'est pas de la compétence de la sociologie, même si elle n'y est pas totalement étrangère.

\section{L'OBSERVATION SCIENTIFIQUE}

Essayons de réfléchir brièvement à la notion d'observation scientifique, définie par opposition aux notions d'expérience (donnée et pratique de laboratoire pour la vérification d'une hypothèse) et d'expérimentation (modification du cours d'un phénomène pour mieux en observer les effets).

Dans le langage de la science, l'observation est un procédé d'investigation grâce auquel le chercheur constate, décrit et mesure, sans le modifier, le mode de production et le déroulement d'un phénomène dans le but d'en déterminer la cause et ses lois.

Il y a observation scientifique lorsque l'observateur a un rapport direct avec la réalité objective. Mais un tel rapport est-il possible?

Par ailleurs, peut-on séparer si nettement l'expérience de l'expérimentation, et l'observation de l'expérience et de l'expérimentation? Il semble que cette séparation/distinction soit irréaliste. Certains travaux de psychologie indiqueraient plutôt que l'observable ne constitue jamais « une simple donnée sensible, passivement enregistrée », mais qu'il est plutôt «le résultat, observé sans présupposition, d'une action expérimentale exercée sur le réel, c'est-à-dire d'une intervention active modifiant l'objet et donnant lieu à une manifestation mesurable. Il y a donc [...] une sorte de délégation de pouvoir de l'observateur en faveur de la mesure, et d'une mesure impliquant une étroite solidarité entre le mesurant et le mesuré.» En plus, «l'observable est directement traduit en symboles opératoires, de caractère mathématique mais entièrement libre à l'égard de la représentation géométrique » (Jean Piaget, Introduction à l'épistémologie génétique. Tome II. La pensée physique, Paris, PUF, 1950, p. 229). 
Si ces recherches psychologiques sont fondées, alors l'observation n'est pas un acte passif, une opération d'enregistrement inerte, mais un processus complexe qui nous permet d'inférer à partir d'une série fragmentaire de sensations auditives, olfactives, tactiles, visuelles, triées au tamis de la culture du savant, passées au sasseur des instruments et des machines du laboratoire.

Norwood Russel Hanson nous rapporte dans son livre Patterns of Discovery les observations, toutes également faites au lever du jour, de l'astronome danois Tycho Brahé (1546-1601) et de l'astronome allemand Johannes Kepler (1571-1630). Pour l'Allemand, le soleil est fixe tandis que la terre tourne sur son orbite. Pour le Danois, la terre est fixe alors que le soleil tourne autour. Les observations de l'un et de l'autre s'appuyaient sur des données visuelles. A partir de l'organisation conceptuelle donnée à des observations du même type, Kepler a conçu ses lois sur le mouvement des planètes autour du soleil, alors que son refus du système héliocentrique du polonais Nicolas Copernic (1473-1543) a amené Brahé à rechercher un compromis avec la doctrine de Ptolémée, l'astronome grec né au $\mathrm{II}^{\mathrm{e}}$ siècle après J.-C., qui affirme que la Terre est immobile au centre de l'Univers.

Les observations deviennent scientifiques grâce à l'organisation conceptuelle construite à l'aide de classifications élaborées à partir de catégorisations linguistiques. Par conséquent, les langages formels et naturels jouent un rôle capital dans les processus de perception-observation-représentation du monde réel, du monde dit positif, tangible, visible. Et également dans les interprétations et dans les significations données aux représentations de ce monde.

Thomas S. Kuhn (1922-1996) a montré que le langage est la condition de possibilité de la science. Deux ou trois chercheurs, travaillant parallèlement sur le même objet, peuvent arriver de façon parfaitement rationnelle à des résultats non équivalents, puisque formulés à l'aide de concepts différents. Et ces résultats peuvent être l'un et l'autre valides, sans pour autant s'équivaloir puisqu'il nous est toujours possible de dire qu'une conclusion est préférable à l'autre. La vérité n'est pas la correspondance entre l'énoncé et une réalité extérieure saisissable de manière unique et unitaire. Il y a plusieurs vérités, mais une seule méthode et une seule rationalité pour les approcher (lire à ce propos l'article de J. Urry, Thomas $S$. Kuhn as Sociologist of Knowledge, in «British Journal of Sociology», XXIV, 1973, n 4, pp. 462-473).

En conclusion, les observations sont représentées grâce à des mots et à des mesures, donc avec des langages. Un langage, qu'il soit formel ou symbolique, ne peut donner qu'une certaine représentation de la réalité ou d'une partie de celleci. L'observation est un processus complexe, c'est une action parmi d'autres actions possibles sur le réel. Il n'y a pas un seul et unique langage pour rendre compte de ce processus. La science est surtout une méthode qui conjugue l'observation avec l'inférence causale et qui donne des résultats différents à des époques où les catégorisations linguistiques sont diverses. Pour cette raison, on n'observe pas de la même manière d'une époque à l'autre puisque les contextes interprétatifs et socio-culturels ne sont plus les mêmes. De cela la sociologie de la connaissance scientifique peut et doit rendre compte. Mais avant d'aller plus loin, ouvrons une parenthèse pour rappeler ce qu'est la nature selon la tradition sociologique. 


\section{LA NATURE SELON L'ANALYSE SOCIOLOGIQUE}

Dans le vocabulaire des sciences sociales, et notamment dans celui de la sociologie, le terme «nature» occupe une place minimale, presque insignifiante. Il est pratiquement impossible, pour les sciences sociales, de reproduire ce que Robin Georges Collingwood (The Ideas of Nature, Oxford, Clarendon Press, 1945) ou R. Lenoble (Esquisse d'une histoire de l'idée de nature, Paris, Albin Michel, 1969) ont fait, par exemple, pour l'histoire de l'idée de nature dans les disciplines philosophiques. Certes, nos enquêtes sur les rapports culture-nature, nos études sur les universaux culturels et la nature humaine sont innombrables et variées; toutefois nous, les sociologues, n'avons jamais fait de la nature un objet d'analyse susceptible d'être circonscrit de façon spécifique et autonome. Une preuve supplémentaire de cet état de fait nous est donnée par les travaux récents s'inspirant soit de la sociobiologie ou de la cybernétique, soit de la théorie des systèmes ou de l'analyse stratégique. Dans ces travaux la nature n'est qu'un objet distant et abstrait, relevant de deux conceptions extrêmes: soit une sorte d'automate autoreproducteur, soit l'infrastructure matérielle de la société.

L'Histoire des mours, publiée sous la direction de Jean Poirier dans 1' «Encyclopédie de la Pléiade », nous donne une présentation magistrale, savante et concise, une mise à jour bien documentée de notre problématique. Il ne me reste donc ici qu'à tenter d'esquisser un rudimentaire état des lieux, d'ébaucher les raisons de l'absence de bonnes théories de la nature en analyse sociologique, de formuler des doutes et des questions, bref, de ne rien dire d'achevé, de nouveau et d'original.

Il est notoire que les pères fondateurs de la sociologie étaient, à une ou deux exceptions près, tous imbus de scientisme et de positivisme. Ils étaient convaincus qu'imiter la méthodologie des sciences naturelles vaudrait à la nouvelle discipline la dignité suprême: celle de science. Adeptes intrépides de l'unité des sciences, les pères fondateurs de la sociologie avaient la certitude que les phénomènes sociaux ne sont guère différents des phénomènes naturels, qu'ils sont des aspects d'une seule et unique réalité. La nature est l'environnement de la société, son support. La nature et la société constituent une totalité intégrée, unique, bien que divisible à l'infini grâce à la méthode analytique. Cette nature-support est une sorte de grand automate soumis à des lois déterministes, à des processus irréversibles, dépourvu de cohérence, de créativité et - bien évidemment - d'une organisation spontanée aux régulations propres. Si dans les écrits sociologiques la référence à la nature reste constante, c'est qu'elle nous donne, à très bon prix, un principe d'intelligibilité semblable à celui en usage dans les sciences dures, un principe capable aussi de légitimer et de hiérarchiser tant d'éléments disparates, contradictoires parfois, régulièrement disjoints. C'est pourquoi les définitions du terme nature, en sociologie, sont rarissimes, alors même que les usages en sont multiples et fréquents. Il dénote et connote à la fois l'ordre primordial nécessaire, a connexion causale fondamentale, la réalité extérieure par rapport à l'intériorité de la conscience, la substance, voire l'essence des faits ou des phénomènes, et en même temps il indique l'équipement psycho-physique au moyen duquel les fonctions sensorielles arrivent à accéder à l'extériorité. Support physique, chimique, biologique du monde social, la nature ne peut être que l'environnement naturel, le milieu extérieur en fonction duquel les hommes se transforment et accroissent les échanges favorables à leur conservation. 
L'outil principal de tous les processus d'adaptation est la société. Celle-ci assure l'équilibre des relations entre les hommes et le milieu avec différents mécanismes assimilateurs ou d'accommodation. Le bon fonctionnement de ces mécanismes est assuré par des règles, des normes, des valeurs, lesquelles fixent les objectifs et les finalités, de même qu'elles légitiment les moyens de les réaliser. Ainsi la société peut gérer aussi bien l'adaptation comme équilibre mobile externe entre le milieu et les hommes, que les fonctionnements invariants de la pensée comme équilibre mobile interne.

Ceci étant donné, la sociologie, dès l'aube de son histoire, va réserver toute son attention et son énergie à trois problématiques réputées majeures. Avec la première, foncièrement déterministe, la sociologie visera à élucider les modalités et l'intensité de l'impact de l'environnement naturel sur les comportements individuels et collectifs, sur les processus sociaux et sur les organisations sociales. Avec la deuxième, elle tentera de prouver que l'action des facteurs naturels est loin d'être mécanique. En nous adaptant, nous nous organisons et en nous organisant nous structurons de plus en plus le milieu. C'est ainsi que les possibilités et les potentialités humaines sont illimitées en variété et en nombre. Avec la troisième problématique, la sociologie essaiera d'éclairer la manière dont la culture, la science et la technique s'ajustent pour favoriser les processus d'adaptation, soit en modifiant l'environnement naturel, soit en dotant les êtres humains d'outils pour les accommodations complémentaires.

Le premier courant de recherches, pour le dire avec les propres termes d'un des meilleurs historiens de la sociologie, Pitirim Sorokin, attribue le rôle prépondérant à «toutes les conditions et [à] tous les phénomènes cosmiques qui existent indépendamment de l'activité de l'homme, qui ne sont pas créés par l'homme, qui changent et varient spontanément, indépendamment de l'existence et de l'activité de l'homme. En d'autres termes, si nous considérons dans sa totalité le milieu d'un homme ou d'un groupe social et en retranchons toutes les influences directement provoquées ou modifiées par l'existence et l'activité de l'homme, il nous restera approximativement ce que l'on nomme le milieu géographique ».

Le deuxième courant tend de plus en plus vers les interprétations de l'environnement naturel comme produit de la culture; il considère cette dernière comme moyen de transformation de la nature.

Enfin, le troisième courant donne la place principale à l'étude des équilibres dans la chaîne d'échanges culture-nature et nature-culture, aux processus d'ajustement entre la culture et l'environnement naturel. Ce courant aboutira dans le constructivisme et finira par accepter - et je l'énonce avec les propres termes de Jean Piaget - que «... l'obligation formelle de transcender sans cesse les systèmes déjà construits pour en assurer la non-contradiction converge avec la tendance génétique à dépasser sans cesse les constructions déjà achevées pour en combler les lacunes...».

Le développement de la recherche empirique va fortement dimensionner l'approche déterministe ou anthropo-géographique. Les corrélations entre les conditions géographiques et l'organisation socio-politique se révèlent ou négatives ou fallacieuses. Des phénomènes sociaux similaires ou équivalents sont observés en des conditions environnementales très différentes, alors que dans des milieux naturels semblables et comparables on trouve des organisations socio-culturelles opposées, ou en tout cas radicalement différenciées. Par contre, les approches 
culturalistes et constructivistes, en leurs différentes versions, attirent fortement l'attention des chercheurs, mais contribuent aussi à éloigner toujours plus la nature de la ligne d'horizon des sciences sociales. Pour les culturalistes de tous bords, il sera prioritaire d'étudier l'impact de l'industrie et de l'art sur les déboisements, sur l'extension des terres cultivées, sur les activités extractives, sur les biotopes, sur l'aménagement et la correction du territoire, sur la domestication des plantes et des animaux. Mais en évaluant le poids de tous ces facteurs qui produisent des transformations substantielles, cette étude a contribué - peut-être sans vraiment le vouloir - à valoriser la thèse selon laquelle l'homme est, d'après la formule célèbre de René Descartes, «maître et possesseur de la nature ». Quant à la position constructiviste, pour qui nature et société constituent une totalité autosuffisante et intégrée, dont les échanges ultimes doivent rester équilibrés afin de consentir leurs reproductions respectives, cette position a été toujours moins importante pour les chercheurs que la position culturaliste.

Le marxisme, surtout celui de la Dialectique de la nature de Friedrich Engels, aurait pu concilier les deux approches. En effet, si l'homme et la société sont constitutifs de la nature, si l'ordre culturel s'institue de concert avec l'ordre social et si l'un et l'autre s'établissent grâce aux interactions, on aurait pu élaborer une doctrine visant à humaniser la nature et à naturaliser les hommes. Cependant, cela ne s'est jamais vérifié. Les marxistes se sont acharnés à mettre surtout en évidence les processus de mutation provoqués partout par les innovations techniques et déterminant successivement les forces et les rapports de production constitutifs de l'infrastructure, ainsi que - dans la superstructure - les institutions sociales en même temps que les idéologies, les philosophies, les représentations de la nature et de la réalité en général. Pour le marxisme l'exploitation de la nature reste inéluctable, mais sans toutefois être létale. En effet, au moment où les hommes institueront une société capable d'abolir toutes les formes d'inégalité, ils mettront alors en place les conditions particulières pour réaliser des échanges hommesnature fonctionnels et équilibrés. Dès lors, il n'y aura plus de question naturelle. Les sociologues, attirés tantôt par l'organicisme etl'évolutionnisme, tantôt par les analyses synchroniques et les conceptions holistiques du fonctionnalisme, ne se sont jamais sérieusement engagés dans l'étude de la problématique marxiste. Ils admettent sans autre que le passé a altéré l'écosystème, que la nature présente est une création des actions humaines passées, que l'équilibre est toujours instable entre les organismes et le milieu, qu'il n'y a pas de résidus extra-humains dans les divers échelons de l'évolution. Pour toutes ces raisons ils préfèrent élucider les divers types de médiations à l'œuvre entre les facteurs physiques, chimiques, biologiques et l'organisation socio-culturelle. Pour ces mêmes raisons ils vont analyser en profondeur les problématiques qui tour à tour font de la culture un habit recouvrant la nudité de la nature, de la culture une partie intégrante de la nature, de la nature le modèle de la culture humaine, et enfin de la nature la culture par définition. Tout en renvoyant ici aux travaux de Alfred Louis Kroeber et de Edmund Ronald Leach pour des analyses exhaustives, je voudrais toutefois y mettre une apostille.

Les débats sur la nature/culture dans les sciences sociales ont fait réapparaître une série de questions qui demeurent toujours sans réponse. Je n'en mentionnerai que deux. La première, signalée en dernier lieu par Claude Lévy-Strauss dans un texte maintenant réimprimé dans Anthropologie structurale deux, peut être 
formulée ainsi: «La conception que beaucoup de sociétés primitives se font du rapport entre la nature et la culture peut aussi exprimer certaines résistances au développement. Celui-ci implique, en effet, une priorité inconditionnelle reconnue à la culture sur la nature... Sans doute la discontinuité entre les deux règnes est-elle universellement reconnue, et il n'est pas de société, si humble soitelle, qui n'accorde une valeur éminente aux arts de la civilisation par la découverte et l'usage desquels l'humanité se sépare de l'animalité. Cependant, chez les peuples dits 'primitifs', la notion de nature offre toujours un caractère ambigu: la nature est pré-culture et elle est aussi sous-culture; mais elle est surtout le terrain sur lequel l'homme peut espérer entrer en contact avec les ancêtres, les esprits et les dieux. Il y a donc dans la notion de nature une composante 'surnaturelle', et cette 'surnature' est aussi incontestablement au-dessus de la culture, que la nature elle-même est en dessous. Dans ces conditions, on ne doit pas s'étonner que les techniques, les objets manufacturés soient affligés dans la pensée indigène d'une sorte de dévalorisation, aussitôt qu'il s'agit de l'essentiel, c'est-à-dire des relations entre l'homme et le monde surnaturel». En est-il encore de même, mutatis mutandis, dans les sociétés de la modernité? Nous n'en savons rien. Jusqu'ici aucune recherche sociologique n'est venue éclairer nos interrogations.

La seconde question se réfère à la problématique dite technique, issue des inventions scientifiques et du travail industriel. Médiation indispensable aux échanges entre la nature et les hommes, la technique de notre temps impose aux transformations une progression incontrôlable. L'électronique, en accroissant nos capacités déductives, modifie nos représentations de la réalité et par là-même nos comportements, voire les processus de formation de ces mêmes comportements. Cette mutation affecte également nos relations avec les autres organismes vivants et avec l'environnement. La mécanisation, à son tour, a bouleversé les modalités d'utilisation des énergies et des informations. Si nous ajoutons à ces constats le fait que les techniques se développent en systèmes, lesquels exigent le recours à l'ingéniérie et ce recours produit d'autres techniques d'une plus grande complexité, il faut alors reconnaître qu'aucun aspect de la vie n'est à l'abri de la technoscience. L'intelligence artificielle (mécanisation + apprentissage), l'automation (mécanisation + contrôle), la robotique sont des manifestions tangibles de cet envahissement, mais aussi une expression puissante de la volonté humaine de tout contrôler, d'utiliser et de manipuler les ressources de la nature, de répliquer l'activité productive humaine en la confiant aux machines. Prolongement des forces naturelles au service de l'homme dans la plupart de ses activités, les machines représentent le stade de la connaissance où les idées de l'homme se reflètent sur lui-même, sur son activité créatrice et sur les processus déterminant son comportement ratiocinateur et conscient, et trouvent une concrétisation techno-scientifique. L'intelligence artificielle et l'automation constituent des systèmes où l'intervention humaine, celle qui s'effectue de l'extérieur, est limitée à la phase d'étude du projet et jamais à celle de l'exécution. Sommes-nous déjà sous l'emprise des artefacts et des systèmes artificiels?

Pour répondre à une telle question, il faudrait au préalable savoir ce qu'est le naturel en nous et dans nos produits, ce qu'est l'artificiel, où, dans l'ordre des activités humaines et de ses produits, on doit situer les artefacts. Mais y a-t-il un critère exact pour distinguer le naturel de l'artificiel? (F. Dagognet, Nature, Paris, Vrin, 1991). Nous considérons comme objets naturels les barrages des castors, les 
toiles tissées par les araignées, les ruches des abeilles, les nids des oiseaux, et nous qualifions pourtant d'artefacts les barrages, les filets de pêche, les paniers, les maisons, les forêts plantées contre les avalanches. Nous considérons naturelle l'évolution produite par des mutations, aléatoire celle qui provient de la sélection et artificielle celle qui est provoquée par le génie génétique. Pourquoi? Il y a, parmi les sociologues, un certain consensus sur la réponse qu'on peut donner à ces interrogations. Convaincus de l'impossibilité qu'il y a à différencier un objet artificiel d'un objet naturel sur la base de la structure, des fonctions et de la morphogenèse, les sociologues pensent que la différenciation ne peut être faite qu'à partir d'un critère anthropocentrique, qui seul permet de présumer que l'un des deux ordres reste indépendant de nous, alors même qu'il est le résultat d'une activité délibérée, celle de l'homme. Pour maintenir la différenciation des deux ordres, le naturel à l'intérieur duquel l'homme s'adapterait spontanément au milieu, et l'artificiel à l'intérieur duquel il transformerait volontairement l'environnement, il faudrait imaginer des processus de transformation du milieu et du processus d'adaptation à ces transformations qui se dérouleraient de façon extra-biologique. Cela paraît pour le moment inconcevable. Par contre nous pouvons affirmer que les artefacts sont de véritables instruments de transformation de la nature et des moyens efficaces pour s'adapter à elle.

Dans la situation présente, nous ne disposons d'aucun critère neutre ou objectif pour distinguer le culturel de l'artificiel. Une telle classification serait uniquement possible relativement à l'interaction entre la nature et l'artificiel dont nous faisons l'expérience à un moment donné. Si, par exemple, nous adoptons un point de vue externe, réceptif, qui nous fait appréhender la nature comme le règne des commandements impératifs ou des empêchements absolus, alors l'artificiel devient un prolongement du monde naturel. Par contre, si nous partons d'un point de vue interne, réactif, qui considère l'homme en tant que sujet d'activités psychiques, conférant du sens aux choses, l'artificiel devient alors partie intégrante de l'humain, de sa volonté, de ses sentiments, donc instrument de l'homme pour la transformation de la nature et moyen de s'adapter à elle. Dans un cas comme dans l'autre, la différenciation est produite de façon anthropocentrique; elle reste la caractéristique d'un système (l'humain) capable de connaître et de s'auto-reconnaître, de faciliter ses rapports à autrui grâce à la médiation de la pensée, de la technique et des institutions sociales. Impossible, donc, de déterminer où s'arrête la nature et où commencent les artefacts sociaux. Le naturel et l'artificiel s'entrecroisent constamment. Pour survivre et nous reproduire, il nous faut transformer la nature, ce que nous faisons au moyen de ces mécanismes de compensation fournis par les modèles culturels, c'est-à-dire avec une évaluation des moyens disponibles, avec des valeurs, avec des normes, avec des symboles, avec des signes.

Ce dépassement de l'activité sensori-motrice a doté l'homme d'une fonction symbolique, qui lui permet de médiatiser n'importe quel type de rapport. Les modèles culturels et les objets de pensée, témoignant des lois de l'activité mentale, sont-ils naturels ou artificiels; sont-ils les produits de l'acquis ou de l'inné? (J. Lopreato, Human nature and Biocultural evolution, Boston, Allen \& Unwin, 1984).

En la matière, les points de vue sont disparates et nombreux. Les plus marquants sont représentés par le constructivisme piagétien, l'innéisme chom- 
skien, le behaviorisme skinnerien, la sociobiologie wilsonienne et, récemment apparues, les sciences cognitives. Ces approches ambitionnent toutes de nous révéler la nature de notre vie mentale. Celle-ci est-elle le reflet de notre culture, de notre histoire personnelle? Est-elle inscrite dans la phylogenèse de l'espèce humaine, dans l'ontogenèse de l'individu, déterminée par les gènes? Une forte proportion de chercheurs penche pour la thèse relative au caractère inné de nos processus fondamentaux. Les recherches actuelles, en effet, visent à prouver que la sémantique aurait des bases neuronales grâce à des mécanismes produisant le codage des représentations mentales. Jacques Mehler et Emmanuel Dupoux, dans leur livre Naître humain (Paris, O. Jacob, 1990), ont rapporté et discuté les plus récentes études expérimentales déterminant les propriétés psychologiques qui, par delà les différences culturelles ou individuelles, sont communes à tous les êtres humains et, en tant que telles, constitutives de la nature humaine.

Les travaux destinés à établir si le nouveau-né a déjà acquis son asymétrie hémisphérique ne se comptent plus. Tous constatent que l'hémisphère droit est d'emblée spécialisé dans la reconnaissance des formes et des visages, alors que l'hémisphère gauche est spécialisé dans le langage, qui doit pourtant être acquis dans un groupe social donné.

Peut-on dire pour autant que l'homme et l'environnement composent un système capable de modifier son programme pour tenir compte des modifications? Une telle thèse, à l'évidence, dissimule un modèle idéologique des rapports de l'homme et de la nature, accordé et stable, comportant des cadres prédéterminés, inscrits dans l'espace, dans le temps et dans les gènes. Ici, faute de place, je ne peux pas en dire davantage. Je préciserai seulement, de la façon la plus concise, que dès 1968 Serge Moscovici a essayé de reposer cette problématique sur de bases nouvelles. Il l'a fait tout d'abord avec l'Essai sur l' histoire humaine de la nature, puis en 1972 avec La société contre-nature et, enfin, en 1974 avec Hommes domestiques et hommes sauvages. Dans ces trois livres, l'opposition de la société et de la nature, l'existence des ordres naturel et social sont vigoureusement contestés.

En quelques mots, la thèse principale de Moscovici peut être résumée ainsi: nous assistons à la fin des sociétés reposant sur l'accumulation interne et sur les conflits de classe, à la fin d'une civilisation. Soumises rigidement au mode d'organisation des rapports entre la collectivité et son environnement, à l'accumulation du pouvoir et de la richesse, ces sociétés ont inventé l'opposition de la société et de la nature, de la pensée noble et du travail servile, de la civilisation et de la technique. Les sociétés nouvelles en train de surgir reposent, tout au contraire, sur la science et la technique; elles sont des cultures et non plus des civilisations, et sont structurées par des activités et non plus par des ordres. Dans ces sociétés la question naturelle remplace la question sociale, car la production des biens y est subordonnée à la création du travail. Selon Moscovici, «L'homme s'affirme sujet de la nature en utilisant ses forces et ses talents à engendrer d'autres talents, des habiletés, des savoirs, et non plus en les appliquant simplement à produire». En effet, dans ces sociétés la vie dépend de plus en plus de la création de ressources naturelles, par la science et la technique, par la programmation et l'organisation.

Cette transformation de l'existence sociale et des mécanismes de développement a fait voler en éclats, selon Moscovici, la conception de la société qui s'oppose à la nature ou vice-versa, comme la forme s'oppose au contenu et la pensée 
à l'objet. Cette conception n'a de vérité qu'historique puisqu'elle correspond à l'idéologie des «sociétés de civilisation». Pour Moscovici l'état du travail détermine non pas directement un type de société, mais d'abord un mode donné de constitution d'une nature. Il n'y a pas de nature en soi, sur laquelle l'homme agirait au moyen de ses outils, puis de ses machines. La nature est un modèle de connaissance et chaque état de nature remplace le précédent à mesure que l'emprise de la connaissance sur la matière s'accroît. Tout appel à une nature, soit comme âge d'or soit comme obscur état primitif, défini hors de la praxis humaine, est purement idéologique et corrompt l'analyse sociologique. Un état naturel est un état de la connaissance, des techniques et aussi de la philosophie, considérée comme théorie de la connaissance et donc aussi de la nature. Un état naturel est un type, c'est un niveau de création. Le travail humain a deux faces: il est élément d'un système d'échanges, d'une organisation économique et partant de rapports sociaux, mais il est aussi et d'abord création et reproduction d'un état naturel.

Bien entendu, Moscovici a essayé de reconstruire ces états naturels et en a particularisé trois: le système organique défini par l'artisanat et la philosophie grecs; le système mécanique qui triomphe de Galilée à Newton; le système cybernétique né avec les sciences expérimentales et la découverte de la chimie et de l'électricité. Ces systèmes sont d'abord des instruments de travail. Le passage d'un état de nature à un autre advient de la manière suivante: l'activité de connaissance suscite la formation, dans un système social donné, de «quasi ressources », dont l'utilisation reste d'abord marginale, mais qui, à la faveur de changements matériels, en particulier démographiques, accroissent les besoins puis se transforment en ressources et en nouvelles forces de production.

La société d'aujourd'hui repose sur la science, la technique et les transformations de la vie sociale. L'image de l'homme reflétant cette société est liée avant tout à la création d'un nouveau milieu naturel, celui de la connaissance scientifique, des produits de synthèse et de l'intervention active dans les processus biologiques.

La transformation de la connaissance et l'apparition de nouvelles catégories naturelles - les savants succédant aux ingénieurs et aux artisans - entraînent l'apparition d'une nouvelle pratique et d'une nouvelle théorie sociale. Elles nous contraignent à redonner la priorité à la création sur l'échange et sur l'organisation, aux forces de production sur les rapports sociaux. Toutes ces raisons font que les problèmes sociaux d'aujourd'hui sont devenus des problèmes naturels; c'est pour ces mêmes raisons que le problème capital, de nos jours, concerne la place de l'homme dans un ensemble naturel continuellement transformé par sa pratique.

Il n'est guère possible d'aborder ici la critique de ces thèses. Je me limiterai à une seule remarque, que voici: je ne sais pas si les thèses de Moscovici sont le produit d'un mirage, celui d'une société créatrice de gigantesques progrès technoscientifiques, capable d'isoler le progrès de la connaissance des valeurs sociales et des valeurs éthiques. Je ne sais pas si ces thèses sont trop marquées par cette forme de néo-saint-simonisme qu'est l'optimisme technocratique contemporain. Je sais par contre que ces thèses sont prises très au sérieux par les psycho-sociologues contemporains. Elles fascinent aussi les théoriciens de l'auto-organisation et tous ceux qui travaillent au croisement de la psycho-chimie, de la biologie, de la cybernétique et des sciences de l'organisation, de l'information et de la communication. Tous ces savants travaillent à l'élaboration d'un nouveau paradigme culturel dont 
les points centraux sont: l'instabilité du chaos, la complémentarité de l'ordre et du désordre, l'autonomie et la capacité de l'organisation nature-société de s'instituer elle-même et de se perpétuer en produisant ses propres lois.

Le livre d'Edgar Morin, Le paradigme perdu paru en 1973, et les volumes de La méthode parus jusqu'à ce jour, nous indiquent une voie pour entrer dans ces nouveaux territoires semés d'embûches et de pièges. En quoi consiste ce nouveau paradigme? A mettre en évidence la logique du vivant. Comment? En dévoilant les articulations entre le naturel et le culturel, entre le biologique et l'anthropologique, en soulignant le fait qu'il y a une seule nature, celle des êtres vivants, fondée sur l'auto-organisation et sur la complexité.

Le paradoxe de ce paradigme qui fait de la vie un système de réorganisation permanente fondé sur la logique de la complexité, a été explicité par Morin luimême en ces termes: «Du coup s'effondre l'ancien paradigme qui opposait nature et culture. L'évolution biologique et l'évolution culturelle sont deux aspects, deux pôles de développement interrelationnés et interférents du phénomène total de l'hominisation: l'évolution biologique, à partir d'un primate intelligent et de sa société déjà complexe se continue en une morphogenèse techno-socio-culturelle laquelle relance et stimule une évolution biologique juvénilisante et cérébralisante... Tout se passe dès lors comme si la société se comportait comme un écosystème social organisateur et organisé, faisant pression sélective et intégrative sur sur les développements ontogénétiques et les mutations génétiques allant dans le sens de la complexité croissante. Le rôle de l'éco-système naturel ne s'en trouve pas annulé. Au contraire, le développement de la complexité sociale établit des relations de plus en plus larges, profondes et complexes avec l'écosystème naturel. Toute économie sociale dépend de plus en plus de l'écologie sociale, tout changement écologique retentit sur l'économie et fait retentir la modification économique sur toute la société. Mais la culture acquiert une relative autonomie, c'est-à-dire qu'une culture complexe ... peut maintenir sa complexité dans un nouveau milieu...».

Certes, l'approche de Morin me semble porter en elle des risques immenses et beaucoup d'incertitudes; elle a néanmoins un mérite incontestable, celui de montrer que le monde est acentrique ou polycentrique et que les rapports naturesociété, culture-nature ne supportent aucune disjonction. Le désordre, tout en demeurant potentiellement dispersion et destruction, reste inséparable de ce qui est création, bref, l'ordre et son organisation sont inséparables du désordre et de la désintégration. Ces thèses, qui restent à vérifier, à contrôler, attirent notre attention sur un point central, à savoir sur le fait que la culture est congénitale à la nature et que la nature est dans la culture. Morin a tiré ces thèses de la thermodynamique, de la théorie des systèmes, de la cybernétique, de la théorie de l'information. Il a essayé de les agencer dans une théorie qu'il appelle «organisationnisme» ou théorie de l'organisation, à partir de laquelle il nous propose une certaine approche des interactions entre la société, la nature et la technologie.

La tentative de Morin m'intrigue et me passionne, mais je doute que nous puissions traiter convenablement un ensemble d'une telle complexité. Néanmoins c'est une question ouverte, à discuter, à approfondir, à laquelle, pour le moment, il est fort malaisé de trouver une solution plus ou moins plausible.

Est-ce que l'approche de Karl Popper et de John Eccles dite des trois mondes (le monde des objets matériels et des façons d'être, le monde de nos états d'esprit 
et le monde des produits de la culture), est-ce que cette approche pourrait mieux rendre compte de notre problématique?

Je ferai à ce propos une très courte remarque: nous ne pouvons guère rendre compte des relations complexes entre ces trois mondes; nous ne disposons même pas d'un langage capable d'énoncer les principes spécifiques et les différentes natures de ces trois entités: (a) physique et physiologique, (b) psychologique, (c) économique et culturelle. Et cette incapacité fait qu'il nous est difficile, voire impossible, de saisir les limites - si limites il y a - entre le monde extérieur, la société et la technologie.

Arrivé à ce point, voici un constat que je peux formuler avec une relative approximation. Toutes nos recherches ne servent qu'à alimenter les controverses d'au moins deux écoles de pensée parfaitement antithétiques : l'une qui célèbre la science et le progrès, l'autre qui professe un naturalisme réactif.

Pour la première école l'exploitation de la nature fait partie de l'ordre normal des choses; elle est inéluctable, mais les techno-sciences seront en mesure d'anéantir tous les effets pervers. L'exploitation contrôlée de la nature est envisageable au nom d'un certain équilibre, de la rareté des ressources, de la variété, de la diversification, du culte du beau, mais il ne s'agit pas là d'impératifs absolus. Certes, les bouleversements techno-scientifiques peuvent produire des modifications importantes dans la vie de notre espèce. Une exploitation contrôlée nous garantirait contre les cassures psychologiques et évolutives, mais elle ne doit d'aucune manière - fixer des frontières à la recherche. Les générations futures sauront bien résoudre les problèmes que nous aurons laissés sans solution. Dans cette optique, développement scientifique et progrès humain sont des absolus, des valeurs fondamentales.

Pour la seconde école il y a antériorité de la nature sur la société. La nature a des droits primordiaux. Elle est la source de la vie et les hommes ne peuvent pas ne pas la respecter, comme par ailleurs ils ne peuvent pas ne pas respecter chaque forme de la vie. Tous les organismes vivants abritent une activité créatrice qui est reliée à une chaîne incommensurable. Dès lors, il est impératif de respecter cette vie et cette créativité, puisque nous partageons la nature avec les animaux et les espèces végétales. Pour cette école, la nature est une valeur en soi; elle lui reconnaît par conséquent des droits inaliénables et fait de l'écosystème, phénomène complexe d'interaction, un sujet moral. On invoque l'interdépendance des espèces et de tous les éléments de l'écosystème (géologiques, physiques, météorologiques, biologiques, etc.) pour rejeter la volonté de domination de la nature par la raison humaine, pour célébrer la capacité de la nature de se reproduire de manière autonome dans le temps. Les tenants de cette école prétendent que les obligations humaines envers la nature dérivent exclusivement de la valeur de la nature en tant que telle, de sa dignité de sujet moral. Dès lors, la nature ne peut plus être un objet d'exploitation, car de son futur harmonieux dépend la survie de notre espèce. Il est donc indispensable de réglementer les interactions entre les hommes et la nature. Cet anti-interventionnisme - propre à certains auteurs devient critique virulente de l'industrialisme au profit d'une science moins envahissante et moins perturbatrice.

L'académicien français Michel Serres a rendu populaires les thèses de cette école grâce à un petit livre intitulé Le contrat naturel, dans lequel il dénonce la prétention des hommes à «pouvoir vivre et penser entre nous pendant que les 
choses obéissantes dormaient, toutes écrasées sous notre emprise: l'histoire des hommes jouissait de soi dans son acosmisme de l'inerte et des autres vivants ». Les contrats sociaux auraient coupé les liens entre les hommes. Une vision exclusivement contractuelle des rapports sociaux aurait atomisé la société civile. Michel Serres écrit: «Par les contrats exclusivement sociaux, nous avons laissé le lien qui nous rattache au monde, celui qui relie le temps qui passe et coule au temps qu'il fait, celui qui met en relation les sciences sociales et celles de l'univers, l'histoire et la géographie, le droit et la nature, la politique et la physique, le lien qui adresse notre langue aux choses muettes, passives, obscures, qui en raison de nos excès reprennent voix, présence, activité, lumière. Nous ne pouvons plus les négliger». D'où la nécessité d'un contrat naturel, statuant la dignité absolue de ce sujet moral qu'est la nature. Mais qu'est-ce qu'un contrat naturel, sinon l'auto-prescription d'une série de restrictions? Est-ce envisageable? Est-ce réalisable? Serions-nous capables, voire pourrions-nous être en mesure de l'honorer? J'en doute.

Un sociologue allemand, Niklas Luhmann, dans une série de travaux au style tortueux et très dense, a montré que le problème nature-société est d'une complexité exceptionnelle et que toutes les solutions proposées jusqu'ici sont inefficaces et irréalistes. Le sociologue de Bielefeld constate que l'environnement naturel est consubstantiel à la société. L'ordre naturel n'est jamais séparé de l'ordre social, bien que, à l'époque contemporaine, ce dernier soit caractérisé par une croissance exponentielle de compétences technologiques lui donnant une emprise presque absolue sur tout. La technique est à la fois un système d'outils et l'utilisation de ceux-ci comme moyens de production primaire, un système assurant la reproduction de ces mêmes outils, un système de distribution du travail entre les hommes, un mécanisme grâce auquel la population se reproduit en tant qu'organisation. La technique est enchâssée dans ce système total fort complexe et autosuffisant qu'est la société. Celle-ci veille à l'intégration fonctionnelle de tous les sous-systèmes, mais il se trouve que chaque élément de cet ensemble est capable d'apprentissage et possède un certain degré d'indétermination dans son fonctionnement spécifique.

Dans le livre Ökologische Kommunikation, paru en 1986, Luhmann va très loin lorsqu'il affirme que les sous-systèmes sont auto-référentiels. Il en résulte que chaque sous-système fonctionne en priorité sur la base de sa propre logique, raison pour laquelle il peut produire des effets pervers par rapport aux autres soussystèmes ou au système global. C'est le cas, notamment, des sous-systèmes techno-science et économie. Ils assurent bien-être et progrès, mais génèrent des risques écologiques comme conséquences non voulues. Pour contrer ces risques, une contre-culture s'est mise à célébrer les vertus de la nature, à la glorifier en tant que sujet moral, à contester la technique et l'économie. Les revendications communautaires les plus passéistes, le rejet de l'industrialisme, la mise en cause du pouvoir de la techno-science sont mariés, dans cette contre-culture, à un hypermodernisme impliquant moult contrôles et restrictions, à une bureaucratisation des rapports sociaux, à une forte limitation de la créativité et des libertés individuelles et collectives. Tout cela peut détruire les sous-systèmes technique et économique. Est-ce que la société moderne pourrait survivre à ce genre de risques, aux menaces écologiques? Pourrait-elle supporter un type de communication sociale risquant d'engendrer un changement radical dans les structures du système de communication de la société en tant qu'ensemble? 
Karl Popper, préoccupé par la renaissance de la métaphysique de la nature (dont Hans Jonas est assurément un des penseurs les plus représentatifs), a lui aussi dénoncé, d'une autre manière, avec sa clarté et sa verve coutumières, les dangers colportés par le naturalisme métaphysique et l'exaltation, au nom d'une éthique sans fondements plausibles, de l'équilibre au détriment de la croissance, de la recherche de l'identité au lieu de la productivité, du vivre, plutôt que du vivre mieux. Bien entendu, Luhmann est beaucoup plus radical que Popper. L'écologie lui semble présenter des options destructrices ou récessives par rapport à la situation actuelle de la communication sociale. Il n'y a présentement, selon lui, aucune possibilité de transférer des informations provenant de la nature au système social. Seul ce dernier est en mesure de ressentir les stimulations, les perturbations, les dérapages ou les menaces. Lui seul peut les interpréter de manière autonome, y donner une suite ou les négliger. Les systèmes sont constitués de relations (d'ordre, de spécification, de production) et sont dotés d'une clôture opérationnelle. La clôture d'un système autopoïétique fait que chaque unité systémique est fermée, séparée, autolimitée. Or la prise en compte du problème de l'environnement ne pourrait que déstabiliser le mode de fonctionnement des unités systémiques. Luhmann considère qu'il s'agit d'une tâche irréalisable pour un système social complexe, caractérisé par le cloisonnement des fonctions des soussystèmes. Il est convaincu que la fonction environnement pousse nos systèmes à se replier sur eux-mêmes en une spirale infinie de communications réciproques incontrôlables, hypercomplexes, hyperbureaucratisées, sans résultats efficients. C'est la raison pour laquelle dans toutes les sociétés avancées la question naturelle a une résonance ou trop forte ou trop faible. Or pour obtenir une résonance efficace, il faudrait que dans les réseaux de structures communicationnelles actuelles la question naturelle soit traitée selon des relations d'équivalence. Ceci n'est guère possible: la société ne peut pas communiquer avec l'environnement, mais seulement au sujet de l'environnement. Et c'est bien la méconnaissance de ce fait, ainsi que la constante augmentation des rapports d'indépendance et d'interdépendance, d'autonomie/subordination, qui suscitent des risques majeurs, dont la culture «verte» est l'expression la plus ambiguë. En effet, l'écologie peut provoquer, sans le vouloir, l'arbitraire, une complexité ingouvernable, des processus normatifs désordonnés, une haute tension entre les sous-systèmes. Elle risque de produire un changement radical des structures du système de communication de la société. Et lorsqu'on sait que le constructivisme absolu de Luhmann postule que la connaissance ne porte pas sur une réalité ontologique, mais exclusivement sur l'ordre et l'organisation des expériences dans le monde de nos mises en œuvre, alors on doit admettre que les événements «existent» uniquement dans la mesure où ils sont traduits en des communications utilisant les codes de chaque soussystème fonctionnel (politique, science, droit, économie, etc.).

Je ne crois pas utile de conclure longuement. Je ne puis que répéter ce que j'ai déjà dit au début: les sciences sociales ne peuvent rien dire sur la nature de la nature. Elles ont rendu plausible la thèse selon laquelle il n'y a pas de nature sans culture, qu'il n'y a pas de culture sans nature. Elles nous ont fait comprendre que cette dichotomie présuppose probablement un troisième terme, toujours implicite ou dissimulé, celui de sur-nature. Les sciences sociales nous ont donné beaucoup de matériaux pour écarter les distinctions simplistes, celles qui, par exemple, mettent d'un côté la matière et de l'autre l'esprit, d'un côté le corps et de l'autre 
l'âme, d'un côté l'inconscient et de l'autre le conscient, d'un côté nos comportements et nos désirs et de l'autre nos rapports aux autres. Elles nous apprennent également que le rapport des hommes à la nature est indissociable des mœurs et des idéologies humaines, des relations des hommes entre eux, des comportements sociaux humains. Elles nous font entrevoir les périls inhérents à des positions qui font de la nature un sujet moral, ou celles qui font de l'homme le maître de l'univers. Elles nous poussent à prendre conscience que - et je veux le dire avec es mots de Cornelius Castoriadis: «... ce qui est requis est plus qu'une 'réforme de l'entendement humain', c'est une réforme de l'être humain en tant qu'être socialhistorique, un ethos de la mortalité, un auto-dépassement de la Raison. Nous n'avons pas besoin de quelques 'sages'. Nous avons besoin que le plus grand nombre acquière et exerce la sagesse - ce qui à son tour requiert une transformation radicale de la société comme société politique, instaurant non seulement la participation formelle mais la passion de tous pour les affaires communes. Or, des êtres humains sages, c'est la dernière chose que la culture actuelle produit. 'Que voulez-vous donc? Changer l'humanité? - Non, quelque chose d'infiniment plus modeste: que l'humanité se change, comme elle l'a déjà fait deux ou trois autres fois'».

Mais que pouvons-nous faire pour placer au centre de nos vies la sagesse pratique, cette rationalité pratique qu'Aristote appelait la phronèsis?

A cette question le sociologue ne peut, hélas! répondre qu'en formulant un vœu: que la collaboration entre spécialistes de sciences humaines, philosophes, juristes et théologiens puisse, enfin, favoriser la naissance d'une «éthique» susceptible de déterminer ce qu'est le bien ou le mal pour l'homme, bref de fournir des repères pouvant aider les hommes à se conduire convenablement dans ce monde complexe et où la «contamination» du milieu semble être une condition nécessaire pour la croissance des libertés individuelles et du bien-être social.

\section{L'ÉCOLOGIE}

L'écologie pourrait-elle nous fournir des matériaux plus solides pour construire une doctrine de la nature qui soit moins susceptible d'entraîner des débats contradictoires?

Les livres sur l'écologie constituent désormais une grande bibliothèque comprenant plusieurs milliers de titres. Il est de plus en plus difficile pour un sociologue de s'orienter dans cette forêt dense, et plus encore de faire le tri entre ce qui est de la propagande, de la politique, de l'idéologie, de la religion, de la philosophie ou de la science.

Frank De Rose et Philippe van Parijs, dans La pensée écologiste. Essai d'inventaire à l'usage de ceux qui la pratiquent comme de ceux qui la craignent (Bruxelles, De Boeck Université, 1993) ont essayé vainement de nous fournir un guide; ils ne sont pas parvenus à dégager les lignes directrices fondamentales de tant d'œuvres, ni par ailleurs à nous proposer des critères interprétatifs satisfaisants. Les travaux d'histoire de l'écologie fournissent, certes, des matériaux abondants, mais ont le défaut de ne jamais analyser préalablement les éléments constitutifs de l'objet de leurs études, d'en fixer avec netteté les contours, si bien que le thématisme finit par en être l'unique fil conducteur. Ils confondent en outre l'his- 
toire des idées et celle des mentalités, et charrient très souvent une philosophie organiciste et holiste (tantôt réductionniste et mécaniste tantôt mystique et religieuse). C'est précisément le cas des travaux de Jean-Marc Drouin (Réinventer la nature. L'écologie et son histoire, Paris, Desclée de Brouwer, 1991), de Jean-Paul Deleage (Histoire de l'écologie. Une science de l' homme et de la nature, Paris, La Découverte, 1991), et surtout du plus célèbre de tous, celui de Donald Worster, Nature's Economy. A History of Ecological Ideas (la première édition a paru en 1977 au Sierra Club Books), traduit en français, fort bien, par Jean-Pierre Denis (Les pionniers de l'écologie. Une histoire des idées écologiques, Paris, Ed. Sang de la Terre, 1992).

La difficulté majeure pour un sociologue de la connaissance scientifique consiste dans le fait que l'histoire de l'écologie n'est pas pareille à celle des autres disciplines. Elle n'est pas une discipline biologique ni un paradigme (écosystémique ou thermodynamique), ni même une façon d'aborder les questions de la crise de l'énergie, de la fin du progrès, de la menace du déclin de la civilisation, de la décadence de l'Occident, de la fin de la planète bleue. De plus, elle se veut le point de rencontre et de fusion des sciences physico-chimiques, humaines et sociales. Modèle de connaissance multidisciplinaire, totale, globale, l'écologie s'arroge le droit d'être la summa de la culture contemporaine, comme le disent explicitement Clarence J. Glacken (Traces of the Rhodian Shore. Nature and Culture in Western Thought from Ancient to the End of the Eighteenth Century, Berkeley, Ca., University of California Press, 1967), et Edward Goldsmith (The Way. An Ecological World View, London, Rider, 1992). Véritable suprascience, l'écologie pourrait enfin concilier la philosophie normative de la science et les recherches sociales empiriques (Steve Fuller, Social Epistemology, Bloomington, Ind., Indiana University Press, 1988).

Les sociologues, pour des raisons qui sont encore à élucider, n'ont jusqu'ici pas beaucoup participé à ces débats. En revanche, les philosophes ont essayé de démêler l'ensemble de la problématique écologique, d'en sérier les thèmes, de clarifier les concepts, de vérifier les fondements argumentatifs de tous les discours des écologistes. Dans ce domaine également, les livres parus ne se comptent plus. Ils ont au moins le mérite de retracer avec précision les transformations du discours sur la nature (voir par exemple le livre publié sous la direction de Jean Greisch, De la nature: de la physique classique au souci écologique, Paris, Beauchesne, 1992), sur l'environnement (Eric Fuchs et Marc Hunyadi, éds, Ethique et natures, Genève, Labor et Fides, 1992), mais aussi sur les animaux (Janine Chanteur, Du droit des bêtes à disposer d'elles-mêmes, Paris, Seuil, 1993), sur les plantes et autres entités naturelles.

Le philosophe Hans Jonas disparu le 5 février 1993, angoissé par l'idée d'une très prochaine catastrophe naturelle provoquée par la technologie, par l'explosion démographique, par l'épuisement des ressources et la contamination du milieu, est allé jusqu'à prôner une métaphysique de la nature imposant une nouvelle éthique de la responsabilité, garantie par un type de régime politique capable de réduire la démographie, la consommation, le gaspillage, et d'imposer une forte discipline sociale vis-à-vis des générations à venir et de la biosphère tout entière.

Un livre de Luc Ferry (Le nouvel ordre écologique. L'arbre, l'animal et l' homme, Paris, Grasset, 1992) aborde avec courage et une pointe d'anticonformisme ces problèmes. Il aurait été excellent si son auteur avait fait une nette 
distinction entre la prétention de l'écologie à être une science, ses implications pratico-politiques, et les visées idéologiques que certains en font découler.

Luc Ferry balance constamment entre l'analyse philosophique des fondements de l'écologie et l'utilisation idéologico-politique qui en est faite. Or, les deux perspectives ne se rencontrent pas nécessairement. Dans l'état actuel de nos connaissances, il n'est pas possible de déterminer si les prétentions de l'écologie à être une science sont fondées en droit et en fait. Par contre, il est possible d'ores et déjà d'évaluer ses arrière-fonds idéologiques, ses présupposés philosophico-politiques. De ce denier point de vue, et uniquement à l'intérieur de la perspective qui est la sienne, le livre de Ferry, malgré une certaine fougue polémique et quelque intempérance verbale, me semble une excellente contribution à l'ouverture d'un débat sérieux sur un problème central de notre vie culturelle d'aujourd'hui.

La thèse principale du livre peut être formulée ainsi: l'écologie constitue une remise en cause de l'humanisme. A l'encontre des valeurs de la modernité et de la démocratie, elle privilégie l'enracinement, la tradition, la nature, et fait l'éloge de la diversité, de la singularité, de la particularité. Les représentants extrémistes de l'écologie vont jusqu'à attribuer à la nature et à toutes les entités naturelles une valeur morale en soi, assortie de droits inaliénables, et rejettent l'anthropocentrisme de la culture occidentale.

Pour Ferry, «la haine des artifices liés à notre civilisation du déracinement est aussi haine de l' humanisme comme tel. Car l'homme est, par excellence, l'être antinature. C'est même sa différence spécifique d'avec les autres êtres, y compris ceux qui semblent les plus proches de lui: les animaux. C'est par là qu'il échappe aux cycles naturels, qu'il accède à la culture, voire à la sphère de la moralité qui suppose un être-pour-la-loi et non seulement pour la nature. C'est parce que l'humanité n'est pas rivée à l'instinct, aux seuls processus biologiques, qu'elle possède une histoire, que les générations se suivent mais ne se ressemblent pas de toute nécessité - là où le règne animal observe une parfaite continuité » (p. 39). Luc Ferry, au fil de toutes les pages de ce livre où la sensibilité se marie admirablement à un savoir étendu, montre l'absurdité de la thèse du contrat naturel et l'ineptie des doctrines conférant aux animaux et aux arbres des droits analogues à ceux des hommes. Je n'ai rien à ajouter à ce qu'il a si bien écrit, sauf peut-être un petit complément. Ce n'est pas la première fois de notre histoire que l'idée des droits des animaux et de la nature est si fortement prisée. Pareto, dans son Traité de sociologie générale, a analysé longuement les thèses affirmant un «droit naturel» des animaux, et a essayé de comprendre comment ce droit arrivait à être reconnu, notamment dans les procès d'animaux que par ailleurs il étudie très attentivement. Le même Pareto montre également que certaines formes extrêmes de la philosophie solidariste du début de notre siècle ancraient ces droits dans les formes de vie communes à l'animal, à la plante et à l'homme. Dès lors, il pose la question: la vie est-elle un critère valable pour légitimer les droits des êtres non humains? Ne serait-ce pas plutôt, en une époque d'affaiblissement des grands systèmes religieux, une manière d'ancrer les croyances à un socle dur, apparemment indiscutable, et se donner ainsi une doctrine des limites de l'agir humain? A ces questions, formulées en d'autres termes, Pareto n'a donné aucune réponse. Janine Chanteur nous a rappelé récemment que seul l'homme a des droits, car lui seul est atteint par l'exigence morale du devoir. Il n'y a pas un droit de la nature, bien qu'il soit parfois indispensable de limiter la cruauté et le saccage pour que les hommes ne régressent point en deçà de l'animalité. 
Luc Ferry, quant à lui, pense que l'antimodernisme des «déconstructeurs de l'Occident en déclin» doit être contré par l'alliance de la démocratie avec l'éthique de l'environnement. Cette alliance sera scellée par la culture humaniste sachant articuler la créativité, l'enracinement et la consommation. La culture n'est pas un simple prolongement de la nature, c'est plutôt l'arrachement aux codes hérités, c'est l'innovation, c'est l'émergence du nouveau, de l'altérité.

Peut-être. Il reste cependant à comprendre pourquoi le «nouvel ordre écologique » a le succès qu'il a alors que l'humanisme démocratique a surtout des partisans silencieux. Cette question n'est pas, j'en conviens volontiers, de type philosophique, mais relève sûrement de la sociologie. Peut-on alors espérer que la lecture du livre de Luc Ferry puisse inciter certains sociologues à s'occuper des idées et des mouvements écologiques? Souhaitons-le, car les enquêtes sociologiques nous aideraient probablement à sortir le débat idéologique du débat métaphysique indécidable dans lequel il s'est présentement abîmé, et ce malgré les efforts méritoires de Bruno Latour pour lui fournir une sortie de sécurité (voir à ce propos Crises des environnements: défis aux sciences humaines, in «Futur antérieur», Nº 6, été 1991, pp. 28-56; Esquisse du parlement des choses, in «Ecologie politique », $\mathrm{N}^{\circ} 10,1994$, pp. 97-107, et Moderniser ou écologiser? A la recherche de la «septième» cité, ibid., $\left.\mathrm{N}^{\circ} 13,1995, \mathrm{pp} .5-27\right)$.

Vous me demandez de vous servir un hors-d'œuvre, c'est-à-dire de dire quelque chose de plus précis sur Serge Moscovici, dont j'ai parlé dans un séminaire précédent. Voici un petit portrait intellectuel de ce savant.

Né à Braïla, en Bessarabie, naturalisé Français, il quitte, en automne 1947, la Roumanie. A près des courts séjours en Autriche et en Italie, il s'établit, en 1948, à Paris où il exerce divers métiers et entreprend des études universitaires à la Sorbonne. Dans la Chronique des années égarées. Récit autobiographique (1997), Moscovici analyse, de manière éblouissante, ses expériences terrifiantes, rapportées néanmoins avec compassion, franchise et perspicacité. Ce récit fait entrevoir la naissance et la formation d'une méthode d'analyse très originale et de théories psychosociologiques fondamentales. Il nous fait également découvrir la genèse des doctrines sur le rôle de la communication langagière et icononique pour la formation et la transmission de la pensée sociale, sur le rôle des phénomènes linguistiques et sémiotiques impliqués dans le fonctionnement intersubjectif de la pensée spontanée, en somme les processus de production et de reproduction du sens commun.

Avec sa thèse de doctorat d'Etat ès lettres, consacrée à la représentation sociale de la psychanalyse (La psychanalyse, son image et son public, 1961), Moscovici explique comment le grand public se représente cette discipline, la modèle et en constitue l'image. La méthode utilisée permet de saisir les modèles psychologiques latents, moyennant lesquels, dans une société donnée, les individus pensent leurs expériences et leurs conduites. Ces modèles sont dénommés les «représentations sociales » et sont utilisés aussi pour critiquer la doctrine selon laquelle l'idéologie influence le monde mental, la culture des masses, la société. Pour Moscovici, un savoir scientifique ou technique devient partie intégrante d'une culture quand il subit une sorte de transformation logique, linguistique et 
culturelle, quand du domaine des spécialistes il passe dans le domaine du sens commun et s'incorpore dans la pensée ordinaire. Mais si les théories scientifiques sont à la fois des récréations de la réalité et des conceptions de l'esprit humain, comment les recherches individuelles et les recherches collectives s'articulent entre elles? Une telle question se retrouve au cœur même de tous les autres travaux de ce chercheur.

L'enquête sur l'image de la psychanalyse est suivie par une série d'autres recherches de terrain, dont les plus marquantes sont Reconversion industrielle et changements sociaux (1961) et Modernisation des mines. Conversion des mineurs (1962). Elles analysent les difficultés pour des ouvriers adultes de passer d'un type d'activité à un autre, d'un système technique traditionnel à une industrialisation aux effets incompréhensibles à la plus part d'entre eux. Le monde du travail est éclairé par l'exploration de la vie personnelle des ouvriers, de leur façon de s'inscrire dans le tissu de la communauté, dans l'histoire. La recherche sur la Chapellerie de l'Aude de 1961 révèle aussi que les villageois ont des pratiques contraceptives, qu'ils sont politiquement progressistes mais demeurent indifférents aux conflits de classes. L'organisation sociale locale fait que les transformations et les changements techniques renforcent les aspects traditionnels de la vie de la sorte qu'ils ne sont pas vécus comme s'il s'agissait de ruptures. La recherche met, en outre, en évidence l'importance des minorité ou des groupes dans les processus de changement social. La recherche sur les charbonnages dans le Midi oblige Moscovici à mettre au point des instruments de mesure, des échelles pour ordonner les questions, pour calculer la différence entre une échelle fournissant des informations et de la redondance et une autre apportant moins d'informations et moins de redondance. Il publie alors une série d'essais remarquables sur les méthodes propres aux enquêtes sociales, sur l'analyse hiérarchique, sur les techniques de construction des échelles, sur la théorie de l'information pour mettre au point des quasi-échelles et des échelles d'attitudes, sur l'analyse factorielle, dimensionnelle, non paramétrique et sur des questions relatives aux techniques d'enquête. Ces outils techniques, illustrés magistralement, évalués à la lumière des résultats obtenus en des divers champs disciplinaires, sont présentés afin qu'ils puissent servir aux chercheurs de terrain, généralement peu experts des procédures de formalisation. Les avantages théoriques et pratiques du langage mathématique pour les sciences de l'homme et de la société, sont présentés clairement et d'une façon magistrale dans l'essai intitulé Pourquoi les mathématiques?

Dans les travaux de la fin des années '60, Moscovici approfondit les problématiques théoriques abordées avec ses premières recherches. De quelle façon la connaissance commune alimente la connaissance scientifique, comment la connaissance scientifique transforme la connaissance commune et quel est le rôle des systèmes de communication dans cette transformation? Quelles sont les relations entre la nature et les sociétés humaines, des hommes face à la culture, à la nature en tant que précondition de la vie? Quel est l'état de la science à une époque donnée et sa façon d'envisager ses relations à la Nature?

Les études retraçant la controverse entre Torricelli et Baliani, reconstruisant les principales étapes de la mécanique prégaliléenne ou les discussions autour de la théorie du mouvement de Michele Vano, ou encore les origines de la mécanique quantique, montrent quelles réponses, dans le passée, on a donné à ces questions. 
Parmi ces études L'expérience du mouvement. Jean-Baptiste Baliani, discipline et critique de Galilée (1967) occupe une place de choix. Cette recherche, basée sur une documentation surtout manuscrite conservée à la Bibliothèque Ambrosienne de Milan, révèle combien incertains étaient, au XVI siècle, les rapports entre les expériences et la théorie, contradictoires les façons d'envisager les rapports à l'univers. Un exemple éloquent est offert, précisément, par les travaux du patricien génois Baliani, né en 1582, à qui on doit une première formulation du principe d'inertie, les premières expériences du thermomètre et ses applications sous le vide. Significatives de l'état de la science à l'époque sont ses discussions avec Galilée, à propos de l'application du modèle mécanique aux problèmes du flux et du reflux des marées, modèle fondé sur l'hypothèse erronée que la lune n'exerce aucune influence sur les marées. La construction théorique de Baliani, conciliant Copernic avec Ptolémée, postule, par contre, que le monde tourne autour de la lune, qu'en conséquence celle-ci exerce une influence mécanique sur les marées. Avec ce travail Moscovici démontre qu'il est difficile d'indiquer le moment précis où une invention est formulée, que l'on sait des choses avant de les nommer, qu'il y a une utilisation de la science avant qu'elle ne soit reprise, théorisée et modélisée par les savants. Après avoir énoncé ces principes, il ébauche une théorie de l'évolution de la science et de la technique et montre qu'au travers d'elles on crée de la nature, des connaissances et des savoirs-faire, des «extra ressources».

Cette théorie est davantage étayée dans l'Essai sur l' histoire humaine de la nature, paru en 1969, où son auteur écrit: «[...] la société est continuellement sortie de la nature, qu'il y a passage incessant de l'une à l'autre. Tous les jours, nos recréons leurs différences et nous déplaçons leurs frontières. Aucune partie de l'humanité, à aucun moment, n'est plus proche ni plus éloignée d'un état de nature, ni dans le passé primitif, ni dans l'avenir évolués ». Le savoir et la façon de voir le monde ne sont pas hiérarchisés. Il n'existe pas de savoir et de technologies qui puissent nous arracher au déterminisme du monde naturel. La nature n'est pas l'environnement, elle est toujours un rapport.

La question naturelle est à nouveau abordée dans La société contre nature, 1972. Le passage du stade de la sauvagerie (ou de l'animalité) au stade de la domestication, est analysé à la suite des théories structuralistes du tabou de l'inceste, du rôle qu'elles attribuent à cette interdiction dans nos sociétés, à l'existence des structures familiales, aux structures de communication linguistiques. Pour Moscovici lorsqu'on cherche le rapport du monde humain au monde animal, le trait distinctif entre l'homme et l'animal, on néglige souvent que le monde animal est aussi un monde social, que le social est une donnée fondamentale dans le monde naturel. Des espèces animales, et en particulier les singes, ont une culture propre. Il y a eu humanisation lorsqu'on est passé des cultures des primates aux cultures humaines, lorsqu'on a transformé les formes de vie, les relations et les règles de comportement. Une réflexion nouvelle sur les concepts de société et de nature, sur l'évolutionnisme et le zoomorphisme, est ainsi amorcée, des nouveaux horizons ouverts, des problèmes anciens reformulés.

Cette réflexion est poursuivie dans Hommes domestiques et hommes sauvages, 1974, où sont étudiés, à l'aide de l'ethnoscience, de l'ethnozoologie, l'ethnobotanique, l'ethnobiologie, les rapports entre la nature et la culture ainsi que les rapports humains et sociaux instaurés par la civilisation industrielle. Le livre est une critique sévère de l'anthropologie symbolique et du marxisme jugés 
inaptes à comprendre que les théories et les idées, pour s'intégrer dans une culture, doivent, préalablement, devenir partie prenante du sens commun. D'autres approfondissements anthropologiques, politiques et socioculturels se trouvent également dans Le ré enchantement du monde. Une écologie politique (2002) et dans De la nature. Pour penser l'écologie (2003).

Ces travaux vont permettre à Moscovici de mieux asseoir sa conception de la psychologie sociale, non plus une spécialisation de la psychologie générale, mais une discipline pont entre différentes branches du savoir jusqu'ici séparées. A la place des traditionnels thèmes de recherches en vogue dès 1950 (Attitudes, Influence sociale et conformisme, Comportements et Décisions de groupes), Moscovici, dès la publication des manuels Introduction à la psychologie sociale (1973) et Psychologie sociale (1984), privilégie les représentations sociales, l'influence sociale minoritaire, les décisions collectives, la psychosociologie de la connaissance. Son approche valorise le social en tant que élément constitutif des processus cognitifs, l'interdépendance du niveau micropsychologique individuel et du niveau macrosocial. A l'encontre de la tradition behavioriste et positiviste, notamment celle des chercheurs américains, il fait valoir que l'individu est un acteur de la vie sociale, agissant avec les autres, dans un monde de significations. Pour comprendre le sens des actions, la connaissance des règles ne suffit pas. Il faut connaître comment les individus organisent leurs expériences dans l'environnement social, comment se développent les dynamiques situationnelles entre les individus, leurs niveaux d'insertion sociétale, les croyances, les valeurs, les idéologies, en bref les processus d'organisation de la vie sociale. Le comportement individuel est foncièrement social puisqu'il se réalise grâce aux échanges et aux negociations des significations, à la fabrication des connaissances. Comme alternative aux doctrines holistes (primauté du social) et aux doctrines individualistes (primauté de l'individu), Moscovici rejette la classique relation binaire sujet-objet et propose son remplacement par une relation à trois termes: sujet individuel (Ego), sujet social (l'Autre), objet. L'explication doit tenir compte des liens entre l'individuel et le collectif, entre le sujet et le système, en leurs genèses, structures et fonctions. Les initiatives et les choix, les possibilités d'action sont les produits des interactions avec les autres, avec les familles, les institutions, les catégories socioprofessionnelles, en somme avec l'environnement social et naturel.

Cette théorie des représentations sociales $(R S)$ fait de la psychologie sociale européenne une alternative, tant théorique que méthodologique, à la psychologie sociale américaine. Les $R S$ sont des systèmes de catégorisation d'aspects du monde, propres à une culture donnée à un moment donné. Ils en favorisent, pour les membres de cette même culture, l'appropriation cognitive collective et leur fournissent une guide pour l'action. La théorie des $R S$ ramène au centre des processus mentaux individuels les processus sociaux et communicationnels entre les groupes et les catégories sociales (Social Représentations. Explorations in Social Psychology, 2001). Les RS se construisent socialement en fonction des phénomènes importants pour l'expérience partagée par la collectivité, même si sur le sens de ces phénomènes il n'y a pas d'identité de vue parmi les différents sous-groupes et les différents acteurs sociaux; elles se construisent dans la vie des groupes, dans les conversations et les échanges qui les fondent; leurs origine se trouve dans le besoin propre à chaque acteur social de donner un sens aux phénomènes inattendus qui mettent en crise les certitudes de la routine quotidienne. En 
d'autres termes, elles reflètent certaines pratiques sociales et déterminent l'apparition des nouvelles.

Le livre Psychologie des minorités actives (1979) est l'aboutissement d'années de recherches expérimentales en laboratoire. En montrant de quelle façon des minorités arrivent à changer les opinions, les manières de faire et de penser des grands ensembles sociaux, son auteur élabore une théorie bien formée de l'influence sociale. Celle-ci rend compte des mécanismes facilitant aux hommes le changement de leurs conceptions et perceptions de la réalité, de leurs comportements, et comment, malgré la pression énorme qu'exerce la société pour les obliger à se conformer au modèle général, aux opinions suggérées par un groupe, par les mass media ou par un personnage doté de pouvoir et de prestige, les minorités et les déviants non seulement résistent mais arrivent même à créer des nouvelles façons de vivre, de penser et d'agir, obligeant de ce fait la majorité à les accepter. Une minorité apparaît lorsque les normes de la majorité deviennent vagues ou connaissent un affaiblissement du consensus. Les minorités actives, pour avoir une influence et transformer la société, doivent se définir par ellesmêmes, d'après leurs propres références, pas d'une manière négative par rapport au groupe majoritaire. Pour cette raison, elles doivent disposer de modèles normatifs, d'une conception des choses et d'elles-mêmes déterminant les comportements, la capacité d'affronter le conflit (moteur du changement) avec la majorité; elles doivent refuser les compromis et exprimer les opinions de manière cohérente, répétitive et sans concessions. Les expériences de laboratoire montrent que l'influence de ces minorités sur les changements de comportements manifestes est nulle, tandis qu'elle est forte sur le plan latent en suscitant des conflits aux répercussions psychologiques sur les opinions. Ces minorités peuvent activer le mental et l'affectif des gens, elles ne peuvent pas leur imposer de solutions, mais tout au plus les aider à y parvenir par eux-mêmes.

Selon Moscovici, l'historique du Mouvement de libération des femmes et des Mouvements écologiques révèlent que leur influence directe a été relativement faible tandis que leurs idées, après une période d'incubation, se sont largement répandues.

Avec L'Age des foules. Un traité historique de psychologie des masses (1981 et 1991) est proposé une reconstruction historique et théorique de la psychologie des masses. Cette discipline, souvent ignorée, a pourtant façonné notre modernité au même titre que l'économie politique. Moscovici procède à une analyse critique du système d'idées constituant la psychologie des foules en discipline cohérente. Les travaux de ses trois plus grands théoriciens, Gustave Le Bon, Gabriel. de Tarde et Sigmund Freud, sont finement analysés ainsi que les trois questions auxquelles ils ont essayé de répondre: Qu'est-ce qui fait agir les masses? Quels hommes sont leurs meneurs et d'où tirent-ils leur puissance? Comment les individus sont-ils entraînés dans les processus de masse? Cet ouvrage illustre les méthodes que la psychologie des foules préconise pour le gouvernement des masses ainsi que leur application, à partir d'exemples empruntés à l'histoire du $\mathrm{XX}^{\mathrm{e}}$ siècle, un peu partout dans le monde. L'ouvrage fait également entrevoir l'expansion des foules en Amérique latine, en Afrique et en Asie et se termine ainsi: «Si la perspective de l'âge des foules est vraie, alors ce livre, consacré à une science classique et à notre passé récent, permettra à ceux qui voudront garder les yeux ouverts de déchiffrer quelques-uns des traits de l'avenir. Un avenir qui est déjà commencé.» 
La théorie des $R S$ ainsi que celle des processus d'influence minoritaire sont reprises dans La machine à faire des dieux. Sociologie et Psychologie (1988; The Invention of Society, 1993) où la question de la croyance et de ses rapports avec la démonstration rationnelle est à nouveau examinée à la lumière de la question: Pourquoi explique-t-on les phénomènes sociaux par d'autres phénomènes sociaux et les phénomènes individuels par des causes psychiques? Il est vrai que les éléments subjectifs, les émotions et les capacités mentales des individus ne déterminent pas la vie en commun, cependant la psyché individuelle est totalement redevable à la société, aux relations entre les hommes et les institutions. En effet, c'est cela à déterminer le contenu et la structure des façons de penser et d'agir de tout un chacun. Mieux, selon Moscovici: «Leurs manière de raisonner, les phrases qu'ils forment, et les habitudes qu'ils ont de marcher ou de sentir proviennent du monde social et y sont incorporés. Que ce soit par tradition ou par apprentissage, elles deviennent des dispositions personnelles, une fois reprises du fond commun.» Tout ce que advient dans une société a son origine dans les passions, dans les sentiments, dans un amalgame d'intérêts et de pensées. Les créations religieuses, politiques et culturelles, qui président à l'être et à l'agir ensemble, sont les produits de sentiments cristallisés en croyances puissantes. Si la démonstration d'une vérité ne pousse pas à l'action, si pour faire agir quelqu'un il faut transformer une idée en croyance, l'enraciner dans la forme de pensée des masses, la rendre irrésistible en la faisant devenir inconsciente, alors il ne faut pas détacher les causes sociales des causes psychiques. La vie sociale est faite de passions (charisme, sacrifice, effervescence, communauté, révolution, etc.). Ces passions gouvernent les mouvements, les crises, les phénomènes où les causes sociales et les causes psychiques se fondent et se confondent. Dès lors il est impératif de ne pas les séparer. Les croyances ne disparaissent jamais. Certaines formes de croyance, religieuses et autres, peuvent s'éteindre, mais la croyance en tant que ciment de la vie collective est éternelle. Aucune vie collective n'est possible si elle n'est pas soutenue et vivifiée par la croyance en son être et en son devenir. Les systèmes de croyances et les modèles culturels sont organisés par les groupes sociaux en représentations sociales, en constructions de la réalité et raisonnements partagés au fil des interactions quotidiennes. La destinée des hommes s'inscrit dans les rapports avec les autres, avec la nature, et évolue à travers les différentes formes d'organisation sociale. Dans le cadre de cette construction théorique, les pages consacrées à Durkheim, Mauss, Weber et Simmel sont d'une finesse interprétative exceptionnelle.

Le livre Dissensions et Consensus. Une théorie générale des décisions collectives (1992) montre que le consensus n'est pas le résultat d'un accord dirigé vers le compromis, la modération rationnelle, le contrôle des informations. Il ne vise pas à éliminer les conflits mais plutôt à transformer les mentalités/identités individuelles et des groupes, à les réaliser moyennant la participation active, des individus et des groupes, aux processus décisionnels collectifs finalisés à la production du consensus.. Au paradigme Risky Shift on oppose celui de Group Polarization. Selon cette doctrine les effets de polarisation dans les décisions collectives de groupe explicitent les mécanismes sociogénétiques qui relient les modalités d'interactions sociales et les changements individuels dans les jugements, les évaluations et les perceptions.

Serge Moscovici travaille actuellement sur l'antisémitisme et le racisme. Il essaie d'aller au delà des limites de la tradition de recherche strictement cogniti- 
viste et d'éclairer les questions suivantes: Pourquoi l'antisémitisme discrimine-til et le racisme extermine-t-il? Comment l'antisémitisme peut-il être racisme?

Dans un univers de plus en plus englobant, caractérisé par des rapports à la nature, aux autres et aux savoirs très compliqués et complexes, dans un monde lié par des phénomènes d'accélération, de sophistications technologiques et d'échanges économiques permanents, dans un monde où la multiplication des conflits locaux est persistante, que faire afin que la civilisation technicienne obsédée par l'économie ne bouleverse plus, et de manière si contraignante, les traditions, les rapports entre les peuples, la participation des citoyens aux décisions culturelles, à ce à quoi ils appartiennent?

Ces interrogations laissent prévoir l'application des théories de la psychologie sociale aux problèmes de la mondialisation, de l'agir politique, aux grandes questions philosophiques et politiques actuelles.

En conclusion, les travaux de Moscovici se caractérisent par leur grande nouveauté; ils ont bouleversé les paradigmes canoniques de la discipline, renouvelé ses méthodes de recherches et ses orientations, crée une tradition européenne en psychologie sociale dont l'originalité et les apports scientifiques et culturels sont reconnus même par les chercheurs partisans de la «Social Cognition». Dans les sciences de l'homme et de la société d'aujourd'hui Serge Moscovici occupe désormais la place d'un Maître éminent, celle même que - jusqu'à la fin des années '60 - a été de Jean Piaget. Il est le fondateur de l'Ecole européenne de psychologie sociale, il est l'auteur d'une œuvre singulière et audacieuse, ses travaux ont renouvelé les méthodes et les orientations de la discipline. Ses théories des représentations sociales, de l'influence sociale des minorités actives, de la pesée des groupes dans les décisions individuelles et collectives, du consensus et des conflits, ont ouvert des perspectives nouvelles, susceptibles de rénover et de renforcer la collaboration interdisciplinaire, la réflexion épistémologique contemporaine, la compréhension des relations entre les hommes, les institutions sociétales et l'histoire humaine de la nature.

\section{SCIENCE ET NON SCIENCE}

J'ai essayé jusqu'ici de mettre en évidence les difficultés contenues dans les concepts d'uniformité de la nature, d'observation de la nature, de théorie vérifiée par les faits. J'ai également essayé de vous expliquer que la nature parle dans la mesure où je la fais parler, que je ne peux la faire parler que si j'ai de bonnes questions à lui poser et seulement si les catégories et les classements de mon langage me permettent d'élaborer ces mêmes questions.

Si les arguments de la thèse que je viens d'esquisser ne sont pas fallacieux, il me faut examiner de plus près l'affirmation selon laquelle il existe des règles qui permettent de discriminer la science de la non science, ce qui est scientifiquement valide de ce qui ne l'est pas. En bref, peut-on dire sans autre que la méthode scientifique (à ne jamais confondre ni avec les techniques d'enquête, ni avec la méthodologie, ni avec l'épistémologie, ni avec les résultats particuliers que cette méthode produit dans certaines circonstances historiques) constitue le critère universel, et le seul, permettant d'identifier la science avec sûreté, puis de la distinguer de la non science? 
Les débats sur les aspects théoriques de la méthode scientifique ont été et sont encore très nombreux. Ma conviction est que ces débats ont surtout porté sur les aspects théoriques de la méthode. Il suffit de se reporter aux travaux d'épistémologie générale ou à Popper, et notamment à son livre de 1969, traduit en français en 1985, Conjectures et Réfutations. La croissance du savoir scientifique, selon lequel le savant, pour rendre la science rationnelle, doit choisir entre des théories différentes. Et la théorie la meilleure est celle qui lui offre le plus grand nombre d'énoncés susceptibles d'être falsifiés.

Popper propose un critère très général, lequel, à mon avis, fait surtout appel à l'expérience que le savant a du métier, à son flair, voire à son feeling. Ce critère, de nature foncièrement floue, est trop général, il ne permet pas d'établir avec précision si l'observation $\mathrm{A}^{\circ \circ}$, ou $\mathrm{A}^{\circ 0 \circ}$, etc., est ou non en accord avec la théorie $\mathrm{A}^{\circ}$. L'application de critères généraux à des observations particulières ne nous donne aucune assurance quant à la pertinence de la démarche, à sa validité. La raison en est évidente. L'observation est elle-même la résultante d'une construction théorique; elle est si chargée de théorie que tous les critères apparemment empiriques en sont façonnés.

Prenons l'exemple de la duplication, dite aussi réplication d'une expérience, d'une expérimentation, d'une observation.

Lorsque l'expérience du savant Un Tel ne se reproduit pas de façon continuelle sous la même forme à l'occasion d'une duplication effectuée par le savant Tel Autre, ce dernier déclare sans fondement, donc inadmissibles, les assertions expérimentales du premier. Les assertions, vraies ou probables, sans contrôle, sont ipso facto déclarées entièrement dépourvues de validité.

Mais peut-on vraiment dupliquer des résultats obtenus en des circonstances de temps et de lieu spécifiques? Le logicien et philosophe Ludwig Wittgenstein (18891951), dans ses Philosophical Investigations, écrites entre 1936 et 1949 mais publiées seulement en 1952 (traduction française 1965), a démontré, et de la façon la plus convaincante, que l'observance d'une règle ne peut jamais être établie sur la base de l'examen de la règle elle-même. En effet, dans une duplication, ou dans une réplication, l'identification des facteurs en jeux découle obligatoirement de l'idée que le savant «duplicateur» se fait des facteurs pouvant intervenir dans la situation d'observation. En plus, la duplication, ou la réplication, n'est pas tributaire des mêmes appareillages et des mêmes systèmes d'organisation, d'où l'impossibilité de reproduire à l'identique une observation, une expérimentation, une assertion. Dès lors, nous sommes obligés d'admettre que n'importe quelle réplication est toujours redevable d'un contexte théorique donné et que ce contexte ne suffit pas à reproduire à l'identique des observations, des expérimentations et des assertions. Celles-ci n'acquièrent des contenus que dans des contextes établis par des constructions théoriques et par les interprétations que ces constructions permettent ensuite d'élaborer.

L'observation du travail des savants montre que dans le cadre d'une même théorie, ils font constamment appel à une multitude de critères ambigus, hétérogènes, irréductibles à des structures formelles. Personne ne peut dire avec précision comment l'on constate l'accord avec le fait, de quoi il s'agit et en quoi cela consiste. La simplicité, la précision, le domaine d'application, la fécondité et l'élégance sont des concepts incertains et imprécis. En outre, leur utilisation change d'une époque à l'autre, d'un contexte à un autre, d'une interprétation à une autre ainsi que d'une communauté de savants à l'autre. 
En d'autres termes, les critères qui permettent l'évaluation des assertions théoriques et des énoncés d'observation prennent des significations différentes suivant les contextes où ils sont utilisés. Par conséquent, il n'est pas prouvé que la véridicité d'une théorie puisse être certifiée par des faits observables. Les faits ne sont jamais indépendants des théories. A supposer qu'il y ait des observations indépendantes de la théorie, affirmer que des observations particulières soient en mesure de prouver l'existence d'un énoncé général, d'une proposition universelle, est pour le moins un paradoxe.

Faut-il alors donner aux théories le statut de simples hypothèses, de conjectures, de suppositions? Voici une question très complexe autour de laquelle le débat reste vif parmi les philosophes contemporains de la science si j'en juge par ce qu'en dit Alan Chalmers dans ses livres Qu'est-ce que la science? et La fabrication de la science.

Un fait toutefois me semble certain: les attributs des critères utilisés pour établir la scientificité d'un énoncé ne sont pas absolus, ils changent d'un contexte à l'autre, d'un savant à l'autre et ils dépendent parfois de la culture technique dominante à un moment donné. La science est un processus d'une complexité extraordinaire, impossible à formaliser rigoureusement et universellement. Mais puis-je pour autant dire que tout cela fait de la science un savoir particulier comme n'importe quel autre savoir social?

Je ne le crois pas. Dans le savoir scientifique, il existe des énoncés stables qui résistent à toutes les nouvelles interprétations. Ces énoncés produisent toujours des conclusions valides et restent compatibles avec les résultats obtenus dans d'autres secteurs de la recherche scientifique. Ils sont là; ils ne sont pas affectés par les contextes et par des facteurs particuliers.

Reconnaître que tous les critères permettant d'identifier la connaissance scientifique ne sont ni invariants ni universels, que dans la pratique scientifique les aménagements et les arrangements locaux sont courants, que les savants interprètent les matériaux expérimentaux en faisant appel aux paramètres et aux ingrédients de leur culture et de leur propre expérience de recherche ne m'autorise nullement à conclure que la connaissance scientifique n'est pas stable, qu'elle est dépendante du contexte social, que les procédures de vérification dont elle dispose n'ont aucune validité universelle. De telles affirmations seraient contraires à la réalité. La gravitation, l'impénétrabilité des corps, l'inertie, la désintégration de la matière, les états solides, liquides, gazeux, etc. sont là pour nous le rappeler.

Certes, on peut soutenir que la science n'est pas une photographie de la nature, que les faits sont construits par les théories, que les observations sont gouvernées par des catégories linguistiques, que le travail scientifique fait appel à des critères variables et parfois indéterminables, que ce travail est fortement redevable aux ressources matérielles et symboliques dont disposent les sociétés, et dire en même temps que le savoir scientifique est un savoir spécifique, très différent de tous les autres savoirs sociaux. Produit dans des sociétés données, sa valeur n'est pourtant pas relative à elles. La valeur et la validité du savoir scientifique dépassent les contextes qui en ont favorisé et permis la production.

Puisqu'il s'agit d'un savoir outrepassant le contexte social-historique, le sociologue doit-il se borner à étudier uniquement le contexte social et laisser à l'épistémologie l'examen des dépassements, des énoncés non reconductibles directement à leurs modes de production? 
La réponse de Merton et de la sociologie classique à cette question ne me paraît ni persuasive ni probante. Les frontières entre la sociologie, l'histoire, l'épistémologie scientifique et la philosophie de la science sont le produit de conventions. Pourquoi le sociologue s'interdirait-il d'étudier, en historien et en épistémologue, ce qui se trouve en deçà ou au-delà du contexte social? Pourquoi s'interdirait-il d'étudier la science comme un fait total dépassant les limites des disciplines académiques? Il n'y a aucune raison pour ne pas le faire.

Récemment, Bruno Latour, dans l'article Philosophie contre sociologie des sciences. Une querelle enfin dépassée? (in «Le Débat», $\mathrm{N}^{\circ} 92$, novembredécembre 1996, pp. 153-163), a relancé la discussion en soulevant une série des questions, par ailleurs déjà esquissées dans Le métier de chercheur. Regard d'un anthropologue, telles que: peut-on réduire le social aux seuls humains, aux seuls intérêts économiques, en excluant les non-humains mobilisés en son sein? Pour quelles raisons négligeons-nous les associations et les combinaisons d'humains et de non-humains?

Latour, convaincu qu' «une société ne se compose pas de sujets de droit en retrait par rapport aux choses, mais de multiples associations et de multiples échanges entre humains et non-humains », donne un rôle actif aux objets, bouleverse la théorie de l'acteur et de l'action et propose un renversement de l'intersubjectivité à l'interobjectivité (voir notamment ses articles Objet d'art, objet de science. Note sur les limites de l'anti-fétichisme, in «Sociologie de l'art», $\mathrm{N}^{\circ} 6$, 1993, pp. 7-24, et Une sociologie sans objet? Remarques sur l'interobjectivité, in «Sociologie du Travail», N 4, 1994, pp. 587-607).

Ces questions sont importantes mais d'une complexité effrayante. Il suffit de lire l'article du physicien anglais John Ziman (Is Science losing its objectivity?, in «Nature», vol. 382, 29 August 1996) ou de parcourir les textes principaux de la polémique suscitée par l'article du professeur Alan D. Sokal (Transgressing the Boundaries: Towards a Transformative Hermeneutics of Quantum Gravity, in «Social Text», Spring/Summer 1996, N 46-47, pp. 217-252) pour en prendre l'exacte mesure. Il m'est impossible ici d'en faire l'historique et de vous en proposer une lecture critique. Pour vous inciter à en prendre connaissance, je vais brièvement vous expliquer de quoi il s'agit.

Le physicien Sokal, intimement choqué par le courant intellectuel qui affirme que la science est affectée par le contexte social dans lequel elle évolue, rédige un canular intitulé «Transgresser les frontières: vers une herméneutique transformatrice de la gravitation quantique » et l'envoie à une revue de sciences sociales de l'Université de Duke, en Caroline du Nord. L'article, en un langage abscons, parsemé d'erreurs et d'inepties patentes, réfute l'idée de l'existence d'un monde externe, «dont les propriétés sont indépendantes de tout individu et même de l'humanité tout entière », et défend la thèse, au moyen d'une érudition à l'esbroufe, que «la 'réalité' physique tout autant que la 'réalité' sociale est, fondamentalement, une construction linguistique et sociale». Toutes les théories qu'on croyaient jusqu'ici constantes et universelles «sont maintenant perçues dans leur inéluctable historicité».

Après la parution de l'article, Sokal révèle (A Physicist Experiment with Cultural Studies, in «Lingua Franca», May-June 1996, pp. 62-64) qu'il a voulu faire une expérience afin d'établir si une revue «publierait un article délibérément saupoudré d'absurdités: a) s'il avait de l'allure, b) s'il flattait les présupposés 
idéologiques de la rédaction. A mon grand regret, la réponse a été oui.» Dès que cette affaire a été connue, les réactions ont été très nombreuses et les débats ont fait rage même dans les colonnes de tous les grands journaux. En consultant Internet, vous trouverez toutes les pièces du dossier. Ici je me bornerai à vous rappeler les suites françaises les plus marquantes de cette affaire.

Denis Duclos (Sokal n' est pas Socrate, «Le Monde», 3 janvier 1997) proteste au nom des intellectuels français contre les attaques américaines. «Elles ne cherchent pas à comprendre mais à parodier, à dénigrer, à piéger, à salir. Leur orchestration relève davantage de l'opération commerciale ou même de la guerre psychologique. Leur philosophie sous-jacente est celle d'un chauvinisme antieuropéen qui tiendrait lieu de commun dénominateur aux zélateurs d'une nouvelle souveraineté, dont nous devrions accepter partout d'être les vassaux, y compris dans la pensée.» Bruno Latour ( $Y$ a-t-il une science après la guerre froide, «Le Monde», 18 janvier 1997), à son tour, soutient que «la blague» Sokal ne prouve rien, n'a aucune portée. Dans un texte paru dans «Libération» du 3 décembre 1996, Latour avait déjà dit que «La blague est drôle, une intervention astucieuse. Elle flanque une bonne raclée à des gens qui la méritent. [Mais pas] aux chercheurs qui, comme moi, font partie des 'science studies' et ont une formation scientifique». Dans l'écrit paru dans «Le Monde», Latour dit que l'essai de Sokal a été publié par une «mauvaise revue». Si l'on en a fait une affaire, c'est que la situation culturelle actuelle laisse sur le carreau les chercheurs qui croient à la science ferme-bouche, à la science «qui permettrait de faire l'impasse sur la vie publique et politique des recherches», à une science qui refuse de passer d'une culture scientiste à une culture de la recherche, qui refuse de tenir compte de la sociologie et de l'histoire sociale.

Pierre Guerlain (Haro français sur le professeur américain, «Le Monde», 14 janvier 1997), quant à lui, rappelle que les «cultural studies» sont en train de déplacer du côté de la sémantique «tous les problèmes sociaux et surtout l'idée post-moderne qu'il n'y a pas de vérité possible, qu'elle n'est que le reflet des rapports de pouvoir à un moment donné, dans un milieu donné». Jean-Jacques Salomon (L'éclat de rire de Sokal, «Le Monde», 31 janvier 1997) ajoute que Sokal n'a fait que répéter une vérité d'évidence: le discours scientifique n'est pas l'équivalent d'un mythe ou d'un poème. Le physicien américain n'a fait que parodier les thèses fallacieuses des tenants du relativisme. «Non pas le relativisme qui soumet à la question les doctrines et les idées reçues, ou le relativisme historique et sa fonction critique, mais celui qui professe que tout est sur le même plan, par exemple, la physique et le chamanisme. Les prétentions à la vérité sont toutes égales, parce qu'au total il n'y a pas de vérité, tant et si bien qu'à force de traiter 'symétriquement' les différentes formes et sources de rationalité, le savoir scientifique n'est plus qu'un récit (ou la technologie qu'un texte) parmi d'autres qui, relevant de l'ethno-anthropologie, renvoie l'épistémologie et l'histoire des sciences au cimetière des disciplines caduques et surtout mystificatrices... Le relativisme, c'est aussi la capacité de dire tout et n'importe quoi, et il faut savoir gré à Sokal» de nous le remémorer.

L'écrivain Michel Rio (Grâce au ciel, à Sokal et à ses pareils, «Le Monde», 11 février 1997) dénonce la «désinvolture ignorante et malpolie» de ceux qui veulent amollir les sciences dures et les littéraliser et il conclut: «Entre l'intellectuel dont la liberté de pensée se fonde sur les contraintes du savoir ou au moins en 
tient compte, serait-il taxé de scientisme, et l'intellectuel-oracle jamais sommé ni désireux ni capable d'étayer le moins du monde ses dires, il se trouve au moins un professionnel de la 'narration' qui penche vers le premier, grâce au ciel, à Sokal et à ses pareils ».

Le physicien Jean Bricmont (La vraie signification de l'affaire Sokal, «Le Monde», 14 janvier 1997) ramène la discussion à son objet véritable. Sokal voulait attirer l'attention sur les dérives du relativisme, sur les extrapolations abusives faites à partir des sciences naturelles et sur les prétentions de certains à parler de la science sans en avoir la moindre connaissance. «Notre but est simplement d'attirer l'attention sur le manque de sérieux et de rigueur dans l'usage qui est fait de la physique et des mathématiques dans des vastes secteurs des sciences humaines. Et il cite, entre autres, à titre d'exemple, «Bruno Latour a écrit un long article où il analyse la théorie de la relativité comme 'contribution à la sociologie de la délégation'» (in «Social Studies of Science», 18, 1988, pp. 3-44).

A Jean Bricmont fait écho Sokal (Pourquoi j'ai écrit ma parodie, «Le Monde», 31 janvier 1997) pour dire qu'il a voulu donner l'estocade aux tendances irrationalistes de notre époque, aux mélanges de l'ontologie et de l'épistémologie, à une sociologie des sciences repliée «sur des platitudes dont personne ne doute». Sokal s'oppose à une conception selon laquelle «l'on peut (et doit) expliquer l'histoire des sciences sans tenir compte de la vérité ou de la fausseté des théories scientifiques. Ce qui veut dire, si l'on est honnête, qu'il faut expliquer l'acceptation des théories de Newton ou de Darwin sans jamais invoquer les preuves empiriques en faveur de ces théories. Passer de cette attitude à l'idée qu'il n'existe pas d'arguments empiriques, ou que ceux-ci sont sans importance, est un pas qui est trop souvent franchi... et qui mène tout droit à l'irrationnel».

Cette polémique nous montre clairement les enjeux auxquels se trouve confrontée la sociologie de la connaissance scientifique, les difficultés qu'elle doit surmonter et les pièges qu'elle doit éviter. La rupture que les «programmes fort et dur» prétendent avoir opérée avec la sociologie traditionnelle des sciences, à la Merton, est un mirage. Il n'en reste pas moins que certains problèmes posés par la «nouvelle sociologie de la science» méritent d'être analysés sereinement, avec impartialité.

Pour une chronique bien informée sur le déroulement du débat aux Etats-Unis, on peut consulter l'article de Loïc Wacquant Les dessus de l' "Affaire Sokal», «Liber», n. 30, mars 1997, pp. 9-10 et n. 31, juin 1997, pp. 14-15.

\section{EN MARGE DES THÈSES DE MERTON, DE BLOOR ET DE GALLINO}

Si je devais résumer de la façon la plus lapidaire possible les arguments développés jusqu'ici, je dirai que ma conception de la sociologie de la connaissance scientifique se trouve à mi-chemin entre les deux positions extrêmes, celle de Merton et celle de Bloor. En effet, je ne partage pas toutes les assomptions de l'Ecole modérée et j'émets les plus grandes réserves envers celles de l'Ecole du programme fort. Mes doutes et mes perplexités vis-à-vis de ces deux Ecoles trouvent leur source dans une série de constats exposés dans les séminaires précédents et que je peux synthétiser ainsi: 
1. Il est impossible de donner une représentation directe du monde physique. La représentation élaborée par la science est toujours plus pauvre que la réalité.

2. L'observation de tel ou tel aspect du monde physique est possible grâce aux catégories linguistiques, aux classements et aux opérations de transformation propres aux langues naturelles et formelles.

3. Les énoncés factuels sont construits par des langages artificiels, formels, ou naturels.

4. L'acceptation des énoncés est redevable de critères variables, parfois indéterminables, qui dépendent des ressources symboliques et du système culturel d'une société donnée.

5. Dans le monde physique on peut trouver des confirmations et des infirmations disparates pour un même énoncé ou pour un ensemble de théories.

6. Les savants peuvent donner différentes interprétations d'une même expérience et d'un énoncé bien établi.

7. Les interprétations sont redevables du contexte socio-historique.

8. La science est un produit social d'un genre particulier. Une fois que l'énoncé est bien établi, il dégage, à certaines conditions, une capacité d'auto-organisation si forte que ses développements ultérieurs peuvent échapper aux contraintes existentielles et sociales.

9. En d'autres termes, la science est d'une part un produit social, et d'autre part un langage construit grâce à des mécanismes relativement indépendants du contexte socio-historique.

10. Dès lors, il est opportun de maintenir, pour le moment, au titre de canon heuristique, les distinctions classiques entre la pensée scientifique et la pensée sociale et historique, entre les sciences naturelles et les sciences historiques. Nous verrons ce dernier point en détail lors de l'analyse des thèses de JeanClaude Passeron.

11. Les difficultés que la sociologie de la connaissance scientifique traverse aujourd'hui dérivent de l'attribution de compétences spécifiques, dans le domaine de la recherche sur la science, à la philosophie, à l'épistémologie, à la méthodologie, à la sociologie et à l'histoire. Or ces divisions, expédients pratiques imposés par les nécessités de l'enseignement, découpent arbitrairement un champ très unitaire.

12. Les différences entre le monde physique et le monde social ne sont pas aussi marquées que notre tradition culturelle et la sociologie de la connaissance classique nous l'ont fait croire. Certes, elles existent et ont une pertinence qu'il serait erroné de négliger ou d'oublier, malgré l'incertitude, le flottement et la précarité des frontières.

13. L'analyse du contexte social de la recherche scientifique a une spécificité et un intérêt dans la mesure où le chercheur ne largue pas les amarres attachant la philosophie à l'histoire et cette dernière à la sociologie. 
14. Pour progresser, la sociologie de la connaissance scientifique ne doit pas renier en bloc le programme dit «modéré ou classique», ni rejeter au complet les thèses du programme dit «fort ou dur».

15. Les tentatives de constituer la philosophie, l'histoire et la sociologie en une discipline nouvelle dénommée «Wissenschaftsforschung», ou «Etudes sur la science », sont à suivre avec attention. Cependant, en l'état actuel, tout inventaire critique me semble prématuré. C'est pour cette raison que je parle des «Etudes sur la science» sans jamais prendre une position argumentée très nette à leur endroit.

Ce résumé ayant été fait, avant de vous parler des problèmes majeurs soulevés par le culturalisme et par l'interprétation culturaliste de la science, il me faut dire quelques mots de la thèse qui fait appel aux technologies informatiques et à l'évolutionnisme bioculturel. Pour cela, je vous propose la lecture du livre du sociologue italien Luciano Gallino, L' incerta alleanza. Modelli di relazione tra scienze umane e scienze della natura (Torino, Einaudi, 1992).

Convaincu de l'équivalence entre science sociale et activité pragmatique, le sociologue de Turin relie implicitement la sociologie à une sorte de méthodologie de l'action, ce qui réduit d'entrée de jeu la science à une compréhension des pratiques humaines et de l'usage du monde destiné à les maîtriser.

L'idée centrale de Gallino est de combler le fossé qui sépare les sciences de la nature des sciences de l'homme et de la société, afin d'unifier le monde de la science et le monde de la vie; il s'agit de construire une éthique scientifique propre à consolider la responsabilité des chercheurs, moyennant une réforme en profondeur des programmes de formation, et d'élaborer une véritable politique de la science ayant le souci de l'environnement et la maîtrise de la technologie.

Le professeur Gallino est persuadé que les sciences naturelles savent résoudre beaucoup de problèmes en raison de leur appareillage conceptuel rigoureux, mais qu'elles sont dépourvues d'une orientation éthique et d'une doctrine des limites socio-politiques, ce dont les sciences humaines sont, par contre, bien dotées. D'où la nécessité d'une coopération loyale entre les deux groupes de disciplines.

Une analyse critique de tous ces thèmes déborderait largement le cadre réservé à un simple séminaire. Je me limite ici à aborder le sujet principal du livre en rapport à mon propos de base: la construction d'un modèle des relations entre les sciences fondé sur la théorie de l'action.

Contrairement à Ilya Prigogine pour qui la «découverte» du temps est en train d'établir une «nouvelle alliance» impliquant l'unification de la science, de la culture et de la société, L. Gallino est de l'avis que cette alliance est incertaine, voire improbable. Les raisons qu'il avance sont simples. La science a oublié qu'elle est une entreprise à la fois cognitive, psychologique et sociale. Elle se veut un tout compact, pur, et nous propose un modèle unique de la réalité et une théorie explicative exclusive qui peuvent légitimer des pratiques dangereuses. De telles prétentions sont redoutables et périlleuses, car aucun principe éthique ou politique ne leur fixe des limites et des compétences. Ces prétentions dérivent d'un défaut constitutif de la gnoséologie occidentale, à savoir la coupure opérée entre le corps et l'esprit, entre le sujet et l'objet, entre les valeurs et les faits. Dès lors, l'esprit détaché du corps s'arroge le pouvoir autonome de former les représentations de la 
réalité. C'est ainsi que la science (simple système adaptatif caractérisé par la production et par la diffusion des connaissances) est devenue capable de prévisions, alors que le social-historique reste aléatoire. Ainsi on a, d'un côté, les enchaînements homogènes des causes et des effets et, de l'autre, les processus fugaces dont on ne sait pas maîtriser les causalités. Cette coupure (dite MindBrain Problem) a perverti les relations entre les sciences naturelles et les sciences humaines, entre la science et la société. Or, selon L. Gallino, ces difficultés pourraient être éliminées grâce à des modèles de relation entre les différentes sciences, et même entre les cultures humaniste et scientifique. La fabrication de ces modèles devrait être le fait d'une science de la science ancrée dans la théorie générale de l'action, dont l'objet serait précisément l'étude analytique et systématique des relations entre les différents groupes disciplinaires.

Pour identifier les connexions entre tous les champs des activités cognitives, cette science de la science s'aventurerait dans le domaine des prédictions empiriques, d'où l'épistémologie est régulièrement absente, et apporterait - vu sa vocation foncièrement pragmatique - son concours aux prises de décision sociales les plus marquantes (technologie, industrie, environnement, santé, éducation, etc.). Telle qu'elle est ici conçue, cette science ne se distingue guère à première vue de la sociologie générale fonctionnaliste et, pas plus que cette dernière, elle n'est en mesure de spécifier les critères significatifs de la validité d'une décision.

L. Gallino considère la science comme un processus qui s'actualise dans un contexte social, tout en produisant des représentations du monde à partir de règles spécifiques. Sans le dire explicitement, il reprend à son compte les propositions formulées par les sociologues partisans du «programme fort» en sociologie de la connaissance scientifique.

L'activité du savant, d'après L. Gallino, est gouvernée par des normes, par des stratégies de défense des modèles du monde propres à sa discipline, par les mécanismes de reproduction des paradigmes constitutifs de son champ disciplinaire. Elle est conditionnée par des buts déterminés et par les moyens disponibles. La production et la diffusion des connaissances sont affectées par ces conditionnements.

Pour Gallino, les choix des savants, la direction de leurs recherches, l'adoption de tel ou tel autre modèle, la divulgation des résultats obtenus, leurs représentations sont autant d'actions orientées par des systèmes et sous-systèmes (culturel, social, psychique, organique, etc.), diversement disposés dans l'espace de l'action. L'acteur-savant opère dans cet espace structuré par les variations de deux paramètres, la contingence et la complexité. L'action du savant est par conséquent le résultat d'une longue et complexe séquence de mouvements dans l'espace de l'action.

Prenons l'exemple du travail théorique, lequel est formé, selon Gallino, de quatre domaines: l'objectivation, la conceptualisation, le finalisme, le pragmatisme.

Le savant doit déterminer une classe d'objets de référence (par ex. gènes, cellules, électrons, etc.), adopter un modèle explicatif (organicisme, mécanicisme, transformisme, etc.), choisir un but (cognitif, pratique, etc.), accomplir certaines opérations (observations, expérimentations, vérifications, etc.).

La mécanique très complexe mise au point permet à L. Gallino d'affirmer que le modèle d'une science dépend essentiellement du domaine que l'on privilégie. 
Les relations interdisciplinaires sont possibles, si on tient compte de cette donnée. Il n'y a pas de modèle de science et de relations interdisciplinaires qui soit bon en soi. Il ne peut l'être que par rapport à l'usage qu'on en fait. Puisque le savant a la liberté d'utiliser tel ou tel modèle selon le but qu'il veut atteindre, sa responsabilité est totale, d'où la nécessité de la soumettre à la surveillance des sciences sociales.

L'ouvrage de Gallino, agencé comme un puzzle très sophistiqué, pose davantage de problèmes qu'il n'en résout véritablement. Certes, l'architecture de ce livre suit des lignes harmonieuses, élégantes et suggestives, mais ses matériaux proviennent tous de l'axiomatique discursive faite de définitions, de postulats et de principes dont on aimerait que la solidité et la validité soient étayées par des preuves empiriques ou des matériaux historiques précis.

Le vieux truisme selon lequel le pragmatisme est foncièrement incapable d'élaborer des théories se confirme une fois de plus. L. Gallino ne précise jamais les rapports logiques et extralogiques réels existant entre les contenus spécifiques des disciplines à mettre en relation. Par ailleurs, aucune des relations évoquées par l'auteur ne parvient vraiment à rendre compte de la spécificité d'une situation. Les prévisions relatives aux développements d'une théorie sont impossibles, tout comme est aléatoire le choix d'une théorie susceptible de résoudre un problème.

Certes, la réalité peut être abordée de différentes façons, et les modèles du monde (concepts, théories, etc.) en représentent les diverses modalités. Cependant, les théories et les concepts ne sont valides et viables que s'ils continuent à produire ce qu'ils sont en mesure de prévoir. Ils disposent donc d'une vie propre qu'il est impossible d'étudier à l'instar des activités socio-politiques et des rapports sociaux dans l'institution scientifique. Les théories sociales, au contraire, ne démontrent jamais rien mais permettent la compréhension de l'agir contingent, imprévisible, indéterminable.

Le livre de L. Gallino réactive, dans un langage moderne, le vieux rêve d'une sociologie capable d'assurer les relations entre des secteurs disciplinaires distincts, de jouer le rôle de mentor de la société juste, et d'être une science unifiée, ou tout au moins intégrée. Il est la preuve que la sociologie ne sait pas faire mieux, en la matière, que la philosophie des sciences, et qu'elle ne sait pas aborder de façon nouvelle les problématiques de la connaissance scientifique.

\section{LA SCIENCE SELON LES CULTURALISTES}

Le culturalisme postule que la personnalité de base, les modèles de comportements des groupes et l'organisation de leurs conduites jouent un rôle déterminant sur les formes et les règles de la connaissance scientifique et des savoirs produits par la science. En conséquence, non seulement les fonctions psychiques individuelles, mais également les phénomènes collectifs et les institutions sociales (la science est une de ces institutions) subissent les influences du facteur culturel.

Selon les culturalistes, la science ainsi que la démarche scientifique (observation, expérience, hypothèse, vérification), systématiquement contrôlées grâce aux structures normatives en vigueur dans la communauté scientifique, n'échappent point aux influences du facteur culturel. L'ethos de la science, tel que Merton l'a décrit, ne serait que de la pure rhétorique sociale. 
$\mathrm{Ne}$ pouvant pas ici passer en revue toute la conception culturaliste de la science, je dois me borner à en discuter les thèses qui me paraissent les plus intéressantes pour la construction d'une sociologie de la science qui voudrait profiter des acquis des théories de l'Ecole de Merton et des théories de l'Ecole de Bloor et Barnes.

Je commence par la question la plus difficile et la plus controversée, la question des découvertes dans les sciences. En parler est aisé puisque nous disposons désormais du travail d'ensemble d'Augustine Brannigan, professeur de sociologie à l'Université de Calgary, au Canada: The Social Basis of Scientific Discoveries, qui est paru en 1981. Cet ouvrage a été traduit en français par Christian Puech (Paris, PUF, 1996) avec une préface de Michael Mulkay, lui aussi professeur de sociologie, mais à l'University de York, en Angleterre, et sympathisant du «programme fort» comme le prouvent assez nettement ses nombreux articles (voir, par exemple, Sociology of Science in the West, in «Current Sociology», $1980, n^{\circ} 3$ ) et ses livres (notamment The Social Process of Innovation, London, Macmillan, 1972, et Science and the Sociology of Knowledge, London, Allen \& Unwin, 1979).

Je vais m'inspirer, très librement et à ma manière, de ce livre de Brannigan, pour vous présenter ce que les culturalistes appellent les fondements sociaux des découvertes scientifiques.

Les théories de l'innovation dans les sciences sont très nombreuses. Des philosophes, des psychologues, des historiens, des sociologues ont essayé de comprendre de quelle façon se font les découvertes. Ils se sont efforcés d'expliquer comment et selon quelles procédures celles-ci sont finalement identifiées, puis étiquetées en tant que telles. Brannigan expose et discute les plus remarquables de ces théories, notamment celles d'orientation foncièrement psychologique de Norwood Russel Hanson, de Richard Blackwell, de Thomas S. Kuhn et d'Arthur Koestler, puis celles d'orientation plus anthropo-sociologique d'Alfred Louis Kroeber et de Robert K. Merton.

Le sociologue canadien partage les théories sur les découvertes scientifiques en deux grandes classes: d'un côté la classe «mentaliste » qui fait de la découverte une réalité réductible aux procédés de représentation mentale, de mémorisation, d'organisation consciente des images et à la logique des idées, et de l'autre la classe «culturaliste » attachée aux conditions sociales de maturation, d'apparition et de reconnaissance de l'innovation, de la découverte.

La tendance mentaliste considère que les découvertes sont le produit de l'activité d'un «génie», ou du changement de la «Gestalt». La tendance culturaliste en fait le résultat des déterminations socio-culturelles.

Les critiques que Brannigan adresse à ces deux courants sont adroites, dans l'ensemble, mais à mon avis, pas toujours pertinentes et constructives. Toutefois, pour notre propos, cela n'a pas une grande importance. Par contre, la conclusion que le sociologue canadien tire de ses critiques est sans aucun doute d'une grande importance. Je peux la résumer ainsi: les découvertes scientifiques n'adviennent pas naturellement. Elles sont le produit d'interprétations, variables et contingentes, élaborées par divers acteurs sociaux. Les découvertes scientifiques sont tributaires de diverses catégorisations, déterminées ou conditionnées socialement, dans des contextes sociaux donnés. C'est précisément pour cette raison que certaines découvertes du passé ne sont plus considérées comme telles aujourd'hui, de 
même que nombre d'inventions et de découvertes contemporaines n'arrivent pas à obtenir, très souvent pour des raisons faciles à élucider, le label si convoité de la reconnaissance à défaut duquel aucune invention n'a pas d'existence véritable.

Brannigan estime que les critères et les mécanismes interprétatifs utilisés, à un certain moment et dans un certain contexte socio-culturel, pour définir, reconnaître et constituer la découverte, sont au nombre de quatre, soit: la possibilité d'une revendication de connaissance, sa validité, le type de motivation en jeu, le degré d'originalité. Lorsque ces critères se trouvent réunis il y a reconnaissance de la découverte, donc il y a découverte.

La découverte ne serait, par conséquent, qu'un procédé interprétatif fait de règles et de pratiques constructivistes, procédé que le savant doit observer pour construire, puis attribuer un sens à un événement, et prétendre à sa reconnaissance en tant que découverte.

Etant donné que l'application d'une règle à un cas particulier pose des problèmes interprétatifs non prévus par la règle elle-même, il arrive que le même procédé, les mêmes règles, les mêmes procédures de construction des événements en tant que découvertes, conduisent les savants à des conclusions antagonistes et contradictoires entre elles.

Avons-nous affaire à des cas d'espèces ou à des phénomènes de nature linguistique?

Dans le premier cas, la réponse est donnée par la reconstruction historique; dans le second cas par la sémantique et l'herméneutique. Nous savons bien que les bonnes raisons susceptibles de justifier une nouvelle interprétation ne sont jamais indépendantes de la raison qui les formule hic et nunc. Cette raison constitue, par conséquent, une autre interprétation qui s'ajoute à la première et la modifie. Les sociologies interprétatives ou herméneutiques nous ont appris que ce genre de processus d'identification peut se prolonger à l'infini, car il reste inachevé à jamais. Puisque ce sont les critères des membres de la société et les usages qu'ils en font qui qualifient la découverte, celle-ci n'est alors qu'une action explicable comme n'importe quelle autre action.

Brannigan prétend que son livre démontre que les découvertes sont des méthodes, mais l'étude des modalités de fonctionnement de telles méthodes n'est jamais véritablement abordée. Michael Mulkay a formulé une remarque intéressante à propos de ce point précis. Je le cite: «Ainsi l'idée est-elle que, une fois que nous aurons compris comment un acte en vient à être jugé comme étant une découverte, ceci nous aidera à expliquer les raisons pour lesquelles il s'ensuit un certain type d'action. Le problème, évidemment, est de savoir comment nous pouvons caractériser ces actions qui s'ensuivent, sans adopter, dans notre traitement des conséquences des découvertes, précisément cette forme d'analyse qui a été abandonnée par rapport à la découverte elle-même. Une fois que l'analyste renonce à l'analyse de la découverte en tant qu'action, pour la remplacer par un examen de la découverte en tant que méthode utilisée par des parties concernées pour construire une action, il semble alors qu'il est obligé de traiter toutes les autres catégories d'action de la même manière. Car il n'existe pas de domaine de la pratique sociale où il est possible d'identifier 'ce qui s'est réellement passé' sans en contrepartie considérer au moins une partie des interprétations par les membres de la société de ce qui s'est passé comme de simples exposés, analytiquement sans problème.» 
Si les critères d'intelligibilité sont multiples, pourquoi alors réduire le comportement novateur uniquement aux seuls facteurs sociaux? Pourquoi adopter un seul modèle explicatif pour un phénomène si complexe et qui se manifeste de différentes manières? Pourquoi réduire la découverte uniquement à sa reconnaissance sociale? Pourquoi négliger la découverte per se et privilégier exclusivement son utilisation «commune par le membre de la société en tant que méthode d'identification et d'élaboration des événements scientifiques »?

L'exemple de Galilée, et par ailleurs de tant d'autres savants au cours de l'histoire, montre qu'il peut y avoir découverte à défaut et au mépris de la reconnaissance sociale, que la signification naturelle de la découverte n'est pas toujours un phénomène populaire et que la médiation des groupes de recherche n'est pas absolument indispensable.

Exclure de l'analyse de la découverte la créativité, c'est-à-dire la capacité d'invention, la capacité d'innovation par laquelle l'action donne une forme nouvelle à une matière préexistante, revient à réduire la découverte à la productivité, pour laquelle la reconnaissance sociale est indispensable. Pour éviter un tel réductionnisme, la sociologie doit faire preuve de modestie en faisant plus souvent appel à l'histoire.

\section{SUR LES FONDEMENTS SOCIAUX DE LA SCIENCE}

Les tenants de la théorie des fondements sociaux de la science réfutent fermement les doctrines qui attribuent à la communauté scientifique la fixation des règles qui sont à la base de l'activité scientifique et des modalités de production des connaissances. Ils contestent l'assomption de Merton et de son Ecole, selon laquelle la structure normative de la communauté des savants impose aux chercheurs d'être détachés, désintéressés, impersonnels, autocritiques, dépourvus de préjugés, neutres, objectifs, soucieux de rechercher la vérité, de dévoiler ce qui est caché et de contribuer aux progrès de la recherche scientifique.

Les culturalistes qualifient toutes ces normes qui caractérisent l'ethos scientifique de pure rhétorique, de cache-misères. Selon eux, la science est un bien symbolique créé par des communautés d'individus, desquelles dépendent les modèles normatifs qui gouvernent les notions de vérité, d'objectivité, de validité ainsi que toutes les autres règles de conduite. De leur point de vue, ces communautés sont les seuls arbitres de ce que sont, à un moment donné, la vérité et la connaissance. Ce sont elles qui établissent si la vérité n'est qu'un rapport de conformité aux faits, ou si elle est le produit de l'usage accepté et reconnu de certaines procédures. A défaut de cette reconnaissance/ratification, il n'y a pas de connaissance scientifique mais uniquement une connaissance pratique. Il n'y a pas de méthode scientifique, pas de science sans communauté scientifique.

Pour les culturalistes, la nature sociale de la connaissance scientifique, la science en tant qu'activité sociale semblable à toutes les autres activités constituent des faits indiscutables, chaque jour confirmés par l'observation empirique. Les culturalistes observent la dépendance des scientifiques vis-à-vis du politique, de l'économie, du militaire, l'importance des idéologies dans les mécanismes producteurs de consensus ou de tensions et de conflits, bref des conditionnements exogènes, des contraintes sociales externes, qui freinent ou accélèrent un progrès 
inéluctable, mettant au service de telle ou telle classe dominante les fruits techniques du progrès. Si la science est conditionnée par la société, si elle en porte les marques et en reflète les contradictions, alors la reconnaissance des découvertes ou même les résistances à l'innovation scientifique ne peuvent se comprendre qu'à l'intérieur de ce cadre.

Dans cette perspective, la science devient un ensemble de modèles de comportement fondés sur un consensus à propos de certains principes fondamentaux (l'objectivité, le préjudice, la vérité, la vérification, etc.). La dimension épistémologique devient un facteur instrumental: c'est le consensus établi par convention sur le sujet/objet, sur la vérité/distorsion, sur la réalité/apparence; quant à la dimension méthodologique, elle représente la structure portante de ce consensus, et donc la charpente même de la communauté scientifique.

Les critiques adressées à la science par les culturalistes sont axées sur l'utilisation des découvertes, sur les investissements, sur le travail fourni par les chercheurs et les techniciens. Elles atteignent leur but dans la mesure où la recherche fondamentale est orientée de la même façon que la recherche appliquée. On ne peut en dire autant des critiques qui visent le contenu même du savoir scientifique, surtout lorsqu'elles conçoivent la science comme un langage plus ou moins arbitraire et qu'elles postulent l'indissolubilité des aspects sociaux et des aspects cognitifs de l'activité scientifique.

Pour associer les aspects sociaux de l'activité scientifique à ses aspects cognitifs, pour prouver que l'activité scientifique est un leurre, une tromperie, voire une imposture, pour affirmer que la science est traversée de part en part par l'idéologie, il est nécessaire de mettre l'accent sur la manière dont les résultats se négocient entre scientifiques, donc sur leurs conflits et sur leurs antagonismes. En d'autres termes, il faut concentrer l'analyse sur les conditions normatives, institutionnelles, socio-politiques qui sont à l'origine de la production d'une connaissance prétendant posséder les caractéristiques de l'objectivité et de l'universalité; il faut démystifier l'autonomie et la neutralité de la science et montrer les accointances des institutions scientifiques avec le complexe militaroindustriel.

Les critiques des culturalistes ne me paraissent pas pertinentes. Elles confondent la dimension analytique et la dimension pragmatique de l'activité scientifique et évaluent ces deux dimensions comme si elles étaient équivalentes ou analogues. Or elles ne sont ni équivalentes ni analogues. Les conditions de validité d'une construction théorique ou non-théorique ne peuvent être confondues avec leurs implications socio-politiques. Certes, l'institutionnalisation et la professionnalisation de la science poussent les savants à participer aux compétitions sociales afin de se procurer de l'autorité et des ressources. Mais peut-on dire pour autant que les critères de validité d'une théorie se réduisent régulièrement et systématiquement aux compétitions sociales?

$\mathrm{Si}$ les règles méthodologiques qui sont à la base de la science sont des conventions, si ces conventions peuvent être décrites comme les règles du jeu de la science empirique, il est alors inévitable de se demander quel est le fondement de ces conventions et de quelle source elles tirent leurs capacités persuasives.

Personne ne peut affirmer que ces conventions sont purement arbitraires. Personne ne peut nier que ces conventions, pour être efficaces et efficientes, doivent être crédibles socialement et utiles pratiquement. Effectivement, elles 
doivent être acceptées par le groupe social des savants, et celui-ci ne les acceptera qu'à certaines conditions.

La première de ces conditions est que ces conventions doivent s'intégrer dans le système cognitif du groupe et qu'elles ne doivent pas entraver la critique. D'autre part, les procédures et les théories acceptées sur la base de ces conventions doivent nécessairement reposer sur des critères méthodologiques. Les critères, les procédures, les théories sont crédibles parce que jugés valides. Le groupe social les accepte et les utilise pour s'orienter parce qu'ils présentent les conditions requises pour produire les effets attendus, parce qu'ils ne sont entachés d'aucune cause de nullité. C'est la validité qui détermine la crédibilité.

Je crois que les culturalistes ne font pas la distinction entre la crédibilité et la validité. Ils négligent le fait que dans les sciences, la validité précède la crédibilité. En bref, ils confondent les deux notions. La validité de certaines connaissances, décidée sur la base de certaines procédures et méthodes, après vérifications et critiques, ne peut être confondue avec un consensus social élaboré dans un contexte déterminé.

Bien sûr, la science peut s'autodécrire comme si elle était un jeu, mais contrairement à ce que l'on peut observer dans d'autres univers symboliques, la science procède volontairement à partir de règles fixées préalablement et moyennant certaines procédures élaborées de façon rationnelle. Pour cette raison, la science ne peut être dogmatique à propos de toutes les règles. Elles sont respectées pour autant qu'elles fonctionnent; elles sont rejetées si elles ne fonctionnent plus ou si elles fonctionnent mal. La science est antidogmatique parce qu'elle est capable de tout mettre en discussion et de tout abandonner, même ses critères les plus éprouvés et primordiaux si l'on constate qu'ils sont devenus inadéquats.

Pour cette raison, je ne peux accepter la réduction de la science et de son analyse critique à cette certitude infondée qui, d'après Wittgenstein, caractérise le jeu linguistique de la vie quotidienne, basé moins sur une proposition infondée que sur un mode d'agir infondé.

$\mathrm{Si}$ les critères analytiques acceptés conventionnellement sont considérés comme des actes politiques imposés par une institution, alors nous réduisons la science à ce que les institutions sociales définissent en tant que telle.

Suffit-il d'être à la tête d'une institution sociale pour produire de la science? Certainement pas. La science vit et se déploie dans des institutions, mais l'agir scientifique n'est pas réductible à la logique de ces mêmes institutions. Il les dépasse, les contourne; il a une logique propre qu'il faut comprendre pleinement avant de la contester et de la nier. J'admets toutefois que nous en savons assez peu à propos des négociations et des interprétations qui caractérisent le déroulement des activités des savants dans les institutions scientifiques. Cette ignorance des processus d'interprétation des ressources culturelles fragilise assurément les reconstructions des modèles normatifs. En l'état nous confondons, ou plutôt nous n'arrivons pas à distinguer convenablement, les règles de conduite des savants dans des situations spécifiques et les normes générales de la science. En outre, nous ne savons presque rien sur les interprétations que les savants donnent de ces règles et de ces normes lorsqu'ils doivent les adapter à des cas particuliers. Mais ces difficultés ne sont point éliminées en établissant un fort lien de causalité entre les normes sociales et la production de la connaissance scientifique. Si nous voulons comprendre les significations contingentes de ces normes et les interpré- 
tations disparates qui en sont données dans des contextes sociaux variables, il faut préalablement établir, moyennant des analyses typologiques, s'il est possible d'observer ou non des uniformités. Sans ces données, la négation de la thèse suivant laquelle la production de la connaissance scientifique dépend de la conformité aux règles formelles propres à la discipline est inacceptable.

Que les savants soumettent continuellement ces règles à des interprétations nouvelles, personne ne le conteste. Mais que ces interprétations constituent des versions personnelles du monde physique et que ce dernier soit reconstruit sur la base des normes sociales dominantes me semble une affirmation peu plausible. Pour accepter une telle thèse, il faudrait établir clairement la liste des facteurs qui exercent une influence sur le contenu de la science et à quel type de contrainte le savant est soumis. Personne n'a pu fournir jusqu'ici les matériaux qui permettraient de fonder ces affirmations. Je ne nie pas qu'il se produise, dans la communauté scientifique, des comportements, des conduites, des conflits, des situations de pouvoir propres à toute activité sociale. Je conteste seulement que ceux-ci déterminent de bout en bout la connaissance scientifique, qu'ils fassent de la science une activité sans autonomie et sans spécificité.

\section{AUTOUR DES REPRÉSENTATIONS SCIENTIFIQUES}

La sociologie de la connaissance a longtemps considéré la science comme un domaine du savoir qui échappait à sa compétence. Elle affirmait - jusqu'au moment où les sociologues ont commencé à s'inspirer de la nouvelle philosophie de la science - que la science dispose d'un statut épistémologique particulier, parce que les propositions et les énoncés des savants sont exclusivement déterminés par le monde physique. En conséquence, la science ne pouvait être qu'une représentation objective du monde. Le contrôle des représentations scientifiques ne pouvait être assuré que par une communauté de savants, agissant de façon foncièrement autonome et en vertu de normes générales, universelles, neutres, désintéressées, établies par des procédures très bien assurées.

Face à cette sociologie de la connaissance se sont dressés des sociologues anglais (par exemple, B. Barnes, D. Bloor, H.M. Collins, M.J. Mulkay, S. Woolgar) et tant d'autres en Allemagne, en France et aux Etats-Unis d'Amérique, pour lesquels la connaissance scientifique est fortement influencée par la Société. Selon eux, la connaissance scientifique est contingente, car affectée par le contexte socio-historique et par les valeurs culturelles d'une époque donnée.

J'essaie de montrer que ces deux conceptions antithétiques de la sociologie de la connaissance, inacceptables à cause de leurs prétentions totalitaires, contiennent, toutefois, chacune des éléments positifs sur lesquels il faut bien réfléchir.

Les sociologues anglais ont posé les bonnes questions à propos de l'observation scientifique, du rapport entre la théorie et la pratique, du principe d'uniformité de la nature et des critères utilisés pour valider la connaissance scientifique. Cependant, les sociologues classiques ont raison d'affirmer qu'il y a une autonomie de la science bien que leur démonstration soit, me semble-t-il, incomplète et par moment trop absolutiste.

Je suis convaincu que les savants élaborent des représentations du monde, avec les outils symboliques et matériels fournis par le système socio-culturel 
d'une époque, mais je pense que ces représentations sont différentes de celles des sciences sociales. Les représentations des sciences sociales construisent et attribuent du sens à des éléments mis ensemble sur la base de déterminations culturelles locales. Ces significations symboliques changent et se modifient dans le temps et dans l'espace, d'où l'impossibilité de les généraliser et de les universaliser. De plus, elles ont affaire à une clôture sémantique incontournable. Par contre, les représentations élaborées par la science trouvent des confirmations ou des infirmations à l'extérieur d'elles-mêmes. Une fois constituées, elles dégagent une capacité d'auto-organisation importante, qui leur procure une certaine autonomie et qui les différencie des autres systèmes symboliques et des autres savoirs sociaux.

Puisque le processus de formation de ces représentations est lié à l'existence d'une métascience et s'effectue dans des situations socio-historiques, le sociologue doit l'analyser, le comprendre et l'expliquer, mais aussi en reconnaître la spécificité. Certes, les représentations scientifiques ne sont pas totalement pures, indépendantes en toutes circonstances des contraintes existentielles et sociales, mais grâce à des procédures formelles, à des langages susceptibles de tenir à distance l'arbitraire social et culturel, à des logiques davantage démonstratives qu'argumentatives, elles acquièrent des propriétés qui les rendent assez souvent universelles, patrimoine commun de cultures différentes. Si ce n'était pas ainsi, pourquoi les lois de la physique, de la biologie ou de la chimie s'imposeraient à des cultures différentes et disparates?

Je maintiens que la science de la nature peut compter, d'une manière ou d'une autre, sur des vérifications extérieures à elle. Ces vérifications donnent lieu parfois à des accords universels et généralisables dans le temps.

Puisque ces vérifications ne sont pas toujours uniques, elles peuvent donner lieu à diverses interprétations et sont à l'origine de significations disparates. Dans ce cas, le sociologue doit être capable de départager l'interprétation, son herméneutique, les implications sociales et les utilisations sociologiques qui peuvent en découler. Les changements observables dans les manières de fabriquer les explications scientifiques ne me paraissent pas être des preuves convaincantes pour soutenir que le monde extérieur n'existe pas, puisqu'il ne détermine pas de façon unique les assertions des savants.

En tant que sociologue, je constate tous les jours qu'il n'y a pas une seule détermination sociale absolue. Ni l'économie, ni la politique, ni la culture, ni la société ne déterminent de manière univoque les divers systèmes d'interactions sociales. Pourquoi présumerais-je que l'univocité est un prédicat essentiel de la nature? Toutes les approches sectorielles et particulières sont, par définition, partiales et réductrices. Mais le jugement sur les approches fausses et ratées doit nécessairement intervenir une fois ou l'autre et de façon définitive, alors que celui sur des approches semblables du système social demeure constamment en instance.

Je reconnais la faiblesse des arguments avancés par la sociologie classique pour défendre le modèle normatif géré par la communauté des savants. Les conduites des savants sont multiples et certaines d'entre elles doivent être rapportées à une culture et à une structure sociale. Les conflits existentiels sont nombreux dans la cité scientifique et il est bon de les connaître et de les analyser. Cependant, ils ne sont pas constitutifs de façon exclusive du noyau dur de la connaissance scientifique. 
Les théories de Galilée ont tenu le cap nonobstant l'opposition de ses collègues et le refus de l'Eglise, puissance dominante à l'époque. Dès lors, le sociologue est bien obligé de départager les aspects cognitifs de l'activité scientifique de ses aspects sociaux.

Je n'ai jamais prétendu que l'activité scientifique soit totalement détachée de la vie en société, ni que les savants soient totalement indépendants des pouvoirs économiques, politiques et sociaux. Par contre, je soutiens que l'activité scientifique et les conduites des savants ne peuvent pas systématiquement être ramenées à ces déterminations et à ces conditionnements. Je prétends que les activités scientifiques dérivent parfois de contextes logiques et d'enchaînements causals fortement structurés et qui n'ont rien à voir avec une société historique.

Pour cette raison, je rejette sans autre les conclusions des sociologues maximalistes qui affirment que la vérité, l'objectivité et la rationalité sont des illusions ou des mirages. Pour cette raison, j'accepte - avec beaucoup de réserves il est vrai - les conclusions des sociologues minimalistes pour lesquels la science arrive à formuler des propositions universelles, pour qui le savoir objectif, fondé sur la rationalité, est possible. Enfin, je m'éloigne des uns et des autres puisque je ne crois pas à l'existence d'une science unifiée. Les sciences naturelles et les sciences sociales traitent de mondes très différents: les unes s'occupent de réalités existant en tant qu'objets extérieurs, relativement indépendants des sujets; les autres de représentations langagières qui construisent la réalité sociale. Comment négliger le fait que celle-ci existe en vertu de la définition que le système de représentation lui a attribuée? Cette réalité ne disparaît-elle pas dès que sa définition est abandonnée ou changée?

Le phlogistique n'avait pas de réalité, même avant la découverte par AntoineLaurent de Lavoisier (1743-1794) du rôle de l'oxygène dans les combustions; tandis que le concubinage, le délit d'initié (qui consiste à utiliser des informations privilégiées pour des opérations en Bourse), le racisme, la tolérance, l'égalité, la liberté, etc., existent en vertu de définitions arbitraires et collectivement admises. Ces faits sociaux disparaissent ou se transforment avec la disparition ou la modification de leurs définitions.

\section{DE LA DÉMONSTRATION A L'ARGUMENTATION}

Depuis que Wilhelm Dilthey (1833-1911) a écrit, notamment dans son Introduction aux sciences de l'Esprit (1883), qu'il y a une grande différence entre les sciences de la nature (qui ont affaire à des objets externes à l'homme, analysables grâce au rapport de cause à effet, soit que l'explication doit déterminer les causes d'un fait, soit qu'elle doit rapporter le fait à la loi externe qui le régit) et les sciences sociales (dont la visée est de comprendre, saisir la signification d'un signe, d'un être et de sa raison d'être), on a pris l'habitude de distinguer l'explication de la compréhension, les sciences physiques des sciences humaines et sociales ou historiques.

L'objectif de Dilthey était de fonder l'autonomie des sciences humaines et de prouver que leurs procédures n'étaient pas réductibles à celles des disciplines naturelles, formelles et expérimentales. Il est inutile d'ajouter qu'une telle distinction a suscité des controverses innombrables. Max Weber, par exemple, attachait 
un prix considérable à la recherche des motifs et des mobiles des actions humaines mais en même temps, il affirmait avec force que seule l'analyse causale (l'imputation causale ou l'observation statistique: régression, dépendance, log-linéaire) pouvait donner à un énoncé la dignité de proposition scientifique. Plus récemment, Jean Piaget a revendiqué l'unité de la science, tout en admettant que l'individu est à la fois sujet de connaissance et objet de sa propre connaissance: «En bref, la difficulté épistémologique centrale des sciences de l'homme étant que ce dernier est à la fois sujet et objet et se prolonge en celle-ci que cet objet étant à son tour un sujet conscient, doué de parole et de multiples symbolismes, l'objectivité et ses conditions préalables de décentration se trouvent rendues d'autant plus difficiles et souvent limitées ».

Je laisse de côté la sociologie phénoménologique, notamment celle d'inspiration heidegerienne, qui postule que le transfert dans les sciences de l'homme des modèles cognitifs des sciences physiques comporte le risque de favoriser la production au détriment de la cognition, la vie active au préjudice de la vie contemplative. Ce courant a soulevé une question capitale, celle de la finalité de la science, argument que nous avons abordé au cours d'un colloque consacré à «l'utilité de la connaissance» et dont les actes ont été publiés en 1988 (De l'utilité de la connaissance, Genève, Droz, 1988).

De nos jours, au nom d'une conception renouvelée de l'unité de la science, le rationalisme critique conteste cette thèse de la différence entre les sciences physiques et les sciences sociales. D'après Hans Albert (1921), dont vous pouvez lire en français un recueil de textes intitulé La sociologie critique en question (Paris, PUF, 1987), cette thèse est foncièrement fausse, du fait qu'elle est basée sur une conception erronée de l'empirisme et du vérificationnisme, sur l'illusion de l'induction et sur le mirage de l'empathie. Pour ce sociologue allemand, l'unité de la démarche scientifique est incontestable. Tout d'abord parce que les frontières entre l'explication et la compréhension sont indécelables, mais aussi parce que toutes les sciences construisent des théories révisables et falsifiables pour rendre compte des phénomènes, et qu'elles doivent forcément procéder à la critique des représentations immédiates des faits externes ainsi que des vécus internes. Ceci est valable pour le physicien des hautes énergies comme pour le sociologue. Si différence il y a entre les sciences physiques et les sciences sociales, celle-ci provient certainement d'un différentiel - temporaire - de développement existant entre les deux blocs de disciplines. L'accumulation des observations et le travail de conceptualisation donneront un jour aux sciences sociales le statut complet de disciplines scientifiques.

Il faut reconnaître que soit la thèse de l'unité, soit celle de la différence/spécificité, sont aujourd'hui sérieusement mises en doute. En effet, l'ontologie fondamentale d'après laquelle un monde extérieur de nature objective existe en soi, indépendamment du sujet connaissant, ne fait plus l'unanimité parmi les chercheurs. Il en va de même de la théorie traditionnelle de la cognition selon laquelle la connaissance du monde extérieur, objectif, s'acquièret à l'aide des moyens empiriques de l'observation et de la vérification.

Il y a de moins en moins de chercheurs qui pensent que la vérité d'une proposition scientifique puisse être prouvée par des procédés et des raisonnements relatifs à l'expérience empirique, au monde extérieur et/ou par la vérification en tant que correspondance de la proposition à un objet ou à un événement du monde 
extérieur. Par ailleurs, beaucoup de réserves ont aussi été avancées à propos de l'observation soi-disant «impossible» des événements passés. Et les physiciens en font de même au sujet des particules élémentaires. Par ailleurs, nous savons que les «Protocol Statements » n'assurent pas la solidité de la vérification, ni l'efficacité de l'observation. En plus, de nombreux chercheurs ont pris conscience du fait que la vérification opère principalement dans un monde d'objets langagiers dotés de significations.

Dès lors, nous sommes confrontés à la question suivante: comment déterminer la signification des concepts fondamentaux? Cette question a été au centre de tous les travaux du Cercle de Vienne, du positivisme logique ainsi que du logicisme de Bertrand Russel. Une intéressante réponse à cette question a été proposée par Ludwig Wittgenstein. En vérité, elle a bouleversé toute la problématique. Le philosophe austro-britannique est arrivé à montrer que le langage n'est pas un moyen de connaissance du monde extérieur, car il en est partie intégrante. Nous nous trouvons donc dans l'impossibilité de faire des inférences de l'un, le langage, à l'autre, le monde extérieur. Par contre, il nous est possible de penser la signification du langage à travers ses usages. Grâce à l'emploi qu'une communauté de parlants fait du langage, maintes difficultés peuvent être éludées. Par exemple, je n'ai pas besoin qu'on m'explique la signification des mots pomme, chat, voiture, gendarme, électricité, argent et ainsi de suite, lorsque les parlants se référent à la pomme fruit, à l'animal chat ou à l'objet mécanique voiture, etc. Il en va de même lorsqu'ils me décrivent un processus quelconque de la réalité extérieure, par exemple le débordement d'une rivière ou le problème de l'intégration des Albanais en Italie ou encore l'électrocoagulation des verrues.

Si nous acceptons le point de vue de Wittgenstein, nous devons reconnaître qu'une quelconque réalité indépendante, physique ou mentale, porte en ellemême les critères et les standards d'un usage correct du langage, mais aussi qu'aucune proposition ne peut être vraie ou fausse en principe, que la question de la vérité est sans solution effective et que les critères de l'usage du langage proviennent avant tout des modèles normatifs à l'œuvre dans la communauté des parlants. En conséquence, si les significations courantes sont des contraintes pour les usagers du langage, alors la communication devient un élément essentiel dans le processus constitutif de la connaissance. Les intentions, les désirs, les états mentaux du parleur, les situations de locution contribuent à donner une signification spécifique aux expressions produites par lui.

En d'autres termes, la conception empirique de la science en tant que discours vérifiable ou falsifiable est rudement mise à l'épreuve par une telle approche, désormais assez courante dans la recherche contemporaine. Aujourd'hui, il y a de bonnes raisons de douter de la thèse selon laquelle le chercheur a la possibilité de déterminer le contenu empirique, direct ou indirect, de chaque assertion scientifique. Mieux, certaines conceptions épistémologiques récentes, notamment celles regroupées sous l'étiquette de post-positivisme, parlent de la connaissance comme d'un produit fabriqué par les membres de la communauté scientifique (ou d'un groupe social), comme d'une pratique sociale ou d'un jeu linguistique gouvernés par des règles et dont le but ultime reste l'acquisition et l'élaboration d'informations sur tel ou tel milieu spécifique d'expérience. Cette connaissanceproduit est composée par un ensemble coordonné d'énoncés (théoriques, conceptuels, évaluatifs, métaphysiques, etc.) qui peuvent guider l'action, orienter une 
pratique à l'aide de modèles normatifs. Précisément pour ces raisons, il y a une constante relation interne entre les règles et les comportements.

Ces recherches ont donné naissance à deux modèles généraux de connaissance, tous les deux abritant par ailleurs des traditions de recherche épistémologique et méthodologique fort différentes.

Le premier modèle est appelé descriptivisme. Il affirme que le langage de la connaissance doit refléter la réalité et la représenter de manière passive et neutre, telle qu'elle est. Il soutient une conception réaliste de la connaissance.

Le second modèle est appelé constructivisme. Il prétend que la connaissance est une œuvre sociale collective, liée au langage, en bref à une pratique sociale qui comporte une intervention active sur la réalité. Pour saisir la réalité et la théoriser, il faut partir d'un point de vue ou d'un schéma conceptuel. Ce sont eux qui nous fournissent une certaine interprétation des objets et des événements qui composent tel ou tel champ de l'expérience. Il est impossible de nous en débarrasser, d'où l'impossibilité d'établir avec certitude si notre langage correspond à la réalité telle qu'elle est effectivement.

Ces deux modèles généraux de connaissance ont ouvert de nouvelles perspectives à la sociologie de la connaissance scientifique. Si la science peut être configurée comme un discours sur des objets, des processus, des interactions, il devient alors essentiel d'étudier les mécanismes et les procédés qui donnent lieu à cette configuration. A présent, les théories de l'argumentation (S. Toulmin, K. Burke, J.-B. Grize, etc.) ainsi que la théorie de l'agir communicationnel (Jürgen Habermas et d'autres) essaient de traiter cette problématique d'une façon renouvelée. Je dirai seulement quelques mots à propos de ces deux courants.

Je fais tout d'abord quelques remarques sur l'argumentation. J'en ai traité dans les Questions actuelles de sociologie de la science (pp. 119-129) comme s'il s'agissait d'un paradigme unitaire, alors que j'aurais dû préciser que je traitais exclusivement des conceptions de Jean-Blaise Grize, lequel oppose la logique naturelle à la logique formelle, l'argumentation à la démonstration. Jean-Blaise Grize, ancien Recteur de l'Université de Neuchâtel, très connu à l'étranger pour ses travaux de logique, est le fondateur, en Suisse, d'une école de recherches qui a mis en évidence l'existence, dans la vie de tous les jours, de logiques, c'est-à-dire de façons de raisonner, très différentes de celles (formelle, symbolique, etc.) utilisées dans les sciences dites dures. En effet, la logique d'un physicien n'est pas la même que celle d'un avocat et celle de l'astronome spécialiste des comètes n'a rien à voir avec celle du garçon faisant la cour à l' «objet aimé », comme disait le père de la bande dessinée, le Genevois Rodolphe Toepffer. Mais pourquoi? Parce que la logique scientifique est impersonnelle; elle élabore surtout des langages abstraits, permettant des calculs et aboutissant à des démonstrations. La logique naturelle, celle de la vie quotidienne, vise à convaincre, traite de choses concrètes et, pour cette raison, ne peut faire mouche qu'avec des arguments fort différents les uns des autres, mais surtout efficaces. Grize, depuis quelques lustres déjà, recherche les lois générales de ces raisonnements argumentatifs. Il s'est consacré à l'étude de la manière par laquelle nous arrivons à convaincre un interlocuteur réticent, indifférent, incertain ou disponible.

Tout le monde sait que les travaux de Grize ont une large diffusion dans les milieux scientifiques européens. Ils suscitent des discussions et des commentaires. A l'heure actuelle Grize est assurément, dans les sciences humaines et 
sociales, le savant suisse le plus connu à l'étranger. Son dernier livre Logique naturelle et communications (Paris, PUF, 1996) est un vrai régal pour les amateurs d'études élégantes. Modèle de concision, de style limpide, d'exposé parfaitement clair, intelligible, ce livre traite d'un sujet qui n'est pas des plus simples, celui de la communication (transmission d'une information, d'un message, d'un signe, d'un signal) dans nos échanges quotidiens.

A la maison, au bureau, au stade, dans la rue, je raisonne et communique, mais je ne démontre presque jamais rien. L'enchaînement de mes propositions n'arrive guère à établir, par exemple, pour un admirateur de Claudia Schiffer la supériorité de la beauté de Naomi Campbell. Pourquoi?

Voici la réponse de Grize: «... nous ne nous contentons pas de produire des suites de propositions, nous échangeons des idées, nous les mettons en commun, nous communiquons dans le cadre social qui est le nôtre ». Mais nous ne le faisons pas de n'importe quelle manière. Nous devons le faire au moyen de certains mécanismes grâce auxquels l'utilisation et la manipulation des mots peuvent produire la persuasion ou le doute, l'adhésion ou le refus, la coopération ou l'opposition.

Dans ce livre magistral Logique naturelle et communications, Grize analyse les mécanismes constitutifs de cette autre logique qu'est la logique naturelle, mécanismes qu'il dénomme «schématisations ». Mais qu'est-ce qu'une schématisation? La schématisation fait voir quelque chose à quelqu'un. En d'autres termes, elle «est une représentation discursive orientée vers un destinataire de ce que son auteur conçoit ou imagine d'une certaine réalité ».

La sagacité avec laquelle, à l'aide d'articles de journaux, de discours de politiciens, d'émissions TV, Grize décrit la mise en forme et en scène de la schématisation est admirable; il s'agit d'un véritable morceau de bravoure. Sans toutefois entrer dans les détails, disons qu'il y a schématisation lorsque le message se présente comme une séquence d'éléments puisés et assemblés selon certaines lois inhérentes à la communication à transmettre au récepteur. Il y a donc schématisation lorsque la séquence d'éléments est commune au couple émetteur-récepteur.

Dans ce livre, on trouve une série de remarques originales, mais trop brèves à mon goût, sur la quantité de variations que l'émetteur peut opérer sans détruire la reconnaissance du type de signe, ainsi que sur la capacité du récepteur de percevoir plus ou moins ces variations.

Communiquer, c'est établir un dialogue entre soi-même et l'environnement social. Phénomène complexe, industrie, marché florissant, idéologie, valeur, la communication est désormais au centre des préoccupations des sociétés démocratiques. Elle est devenue une discipline à la mode. Régis Debray y exerce ses talents versatiles et multiformes ainsi que Dominique Wolton ses capacités extraordinaires de metteur en scène de la culture de pointe (voir à ce propos son recueil de textes Argumentation et rhétorique, Paris, CNRS, 1996, 2 vols.). Jean-Blaise Grize, avec sa simplicité et sa modestie habituelles, nous introduit, quant à lui, à «la problématique de l'argumentation, celle des signes, celle de la communication et de la logique naturelle qui les dirige toutes ». Il remet en question des idées reçues, la vision ordinaire de nos échanges quotidiens, l'actualité, ainsi que la transparence et la véridicité des systèmes de communication.

En refermant ce livre particulièrement clair à propos d'un sujet complexe, j'ai également appris, paradoxalement, qu'il est désormais indispensable d'appliquer, 
au même titre, les découvertes de Grize aux discours politiques, aux moyens de communication et de connaissance, piliers fondamentaux de la démocratie.

Pour être plus exhaustif sur la théorie de l'argumentation, il faut ajouter que tout discours se déroule dans le contexte d'une interaction, structurée par des représentations communes, entre celui qui argumente et celui auquel cette argumentation s'adresse.

Le sociologue n'ignore point que les chercheurs en théorie de l'argumentation décrivent, ou reconstruisent, tantôt le contenu des argumentations et leurs constantes formelles, tantôt la validité et l'efficacité des arguments utilisés. Il sait également que les constructions théoriques sont élaborées avec des langues formelles, lesquelles sont des instruments de calcul sans sujet et ne peuvent en aucune manière servir de métalangue, alors que le langues naturelles sont des instruments de dialogue entre des interlocuteurs et peuvent servir de métalangue. En outre, il sait que le savant fabrique aussi des constructions non-théoriques au moyen des langues naturelles. C'est pour cette raison qu'il lui est possible d'étudier, en plus des conditionnements sociaux de l'activité scientifique et des processus d'institutionnalisation de l'activité scientifique, aussi toutes les constructions non-théoriques des savants, notamment quand ils doivent recourir à des pré-construits culturels, à la polysémie des concepts, aux polymorphismes des énoncés, à la métalangue, pour arriver à construire des expressions bien formées et finalement passer à l'axiomatisation et au calcul, à la logique formelle.

Dans les sciences, les objets construits à l'aide de la logique formelle peuvent être différenciés des objets construits à l'aide de la logique naturelle. Mais qu'en est-il en sociologie et dans les sciences sociales en général?

Dans les sciences sociales, il est impossible de construire des objets simples, à la façon de la physique. Tout découpage nous renvoie à un contexte et celui-ci à quelque chose de plus englobant. Les conceptualisations afférentes à ces découpages et aux définitions interprétatives ne relèvent ni des langues formelles ni entièrement des langues naturelles, ni du raisonnement expérimental ni de l'herméneutique. Selon Jean-Claude Passeron, les constructions sociologiques se placent à égale distance de l'axiomatisation scientifique et de l'argumentation naturelle, elles seraient le produit $d^{\prime}$ ' un raisonnement entre deux ». Le raisonnement sociologique appartiendrait à l'«espace non-poppérien du raisonnement naturel».

Ce raisonnement utiliserait un principe de rationalité très particulier, à propos duquel Passeron a écrit des pages d'une grande perspicacité et sur lesquelles nous reviendrons dans les semaines à venir.

Pour ce qui concerne la théorie de l' «agir communicationnel», vous pourrez consulter l'excellent livre de Jean-Marc Ferry, Habermas : l'éthique de la communication (Paris, PUF, 1987), présentation minutieuse et critique des théories du sociologue-philosophe allemand. Ici, je me bornerai à faire quelques remarques rapides (pour de plus amples développements voir G. Dahl, Critique, Conservatism, Ideology: The Frankfurt School. Critique of the Sociology of Knowledge Revisited, in «Current Perspective in Social Theory», 1995, n. 15, pp. 155-179, ainsi que R. Boudon, L'art de se persuader des idées douteuses, fragiles ou fausses, Paris, Seuil, 1992).

L'analyse des rapports entre connaissance et intérêt amène Habermas à rejeter sans appel le modèle positiviste de la connaissance. 
Le positivisme donne, selon Habermas, une place prépondérante aux savoirs techniques, au calcul et à la raison instrumentale dans le but de reproduire les choses telles qu'elles sont et de propager l'illusion objectiviste. Il efface tous les modèles de connaissance visant à mettre en évidence les autres intérêts constitutifs des savoirs, par exemple les savoirs interprétatifs, qui refusent l'unité de la science et accordent la plus grande importance au sens, aux significations ou encore les savoirs critiques et émancipateurs liant la connaissance et la raison aux universaux et aux intérêts fondamentaux des hommes et des sociétés.

Contre les prétentions du positivisme et du scientisme qui affirment que toute la connaissance est produite par les sciences naturelles et exactes, Habermas revendique aussi une telle dignité pour les sciences de l'homme et de la société. Dans le sillon de la tradition néo-kantienne, Habermas ajoute à l'explication causaliste des sciences de la nature, la compréhension herméneutique des sciences socio-historiques. L'épistémologie des sciences de l'homme et de la société n'est pas et ne doit pas être le duplicata, l'imitation de celle des sciences naturelles ou la conceptualisation critique de la méthodologie des sciences exactes. Selon Habermas, il y a trois types de sciences basées sur trois types d'intérêts:

1. Les sciences empirico-analytiques, qui produisent des savoirs prédictifs débouchant sur des applications techniques. L'intérêt technique commande ces sciences. Elles ont le même cadre de référence que la technique (histoire linéaire et cumulative, objectivisation moyennant des appareillages). Les sciences et les techniques sont des activités instrumentales. Elles s'inscrivent dans la structure même du travail humain.

2. Les sciences historico-herméneutiques qui assurent la communication entre le présent et le passé grâce à l'interprétation. Dans ces sciences, l'intérêt pratique est prépondérant.

3. Les sciences critiques qui éclairent les sociétés à partir de ce qu'elles ne sont pas encore. L'intérêt émancipateur est à la base de ces sciences-là.

Les intérêts qui sont à la base de la connaissance ne sont pas de nature empirique, ni de nature psychologique, ni socio-historiques. Ce sont des quasi-invariants anthropologiques, formés au cours de l'évolution de l'espèce humaine, et bien évidemment façonnés par l'histoire. Habermas attribue à ces quasi-invariants anthropologiques un statut quasi-transcendantal, un statut constituant ou exprimant, pour ainsi dire, une sorte de condition a priori de l'expérience humaine.

Si l'intérêt technique renvoie au travail, l'intérêt pratique et l'intérêt émancipateur renvoient à l'interaction (action et réaction interpersonnelles, influence mutuelle et réciproque dans un système de personnes ou de partenaires sociaux). Or l'interaction est le principe et le résultat des échanges verbaux, elle nous ramène donc au langage, à la communication, à une situation où le comprendre et le faire comprendre postulent que les actes de parole soient fondés sur une confiance réciproque. Cette confiance réciproque est possible pour autant que les acteurs sociaux se conforment aux exigences universelles de la vérité, de la légitimité normative et de la sincérité.

Comment peut-on constater une telle conformité? D'après Habermas, chaque sujet social est doté d'une compréhension intuitive de la conformité, des entorses, 
des différences ou de la déviance produites par les manières de respecter ces exigences universelles. La force des arguments (par analogie, autorité, causalité, comparaison, concession, par les conséquences, etc.), l'argumentation (faire croire, sans aboutir nécessairement à faire faire) sont les seules règles de la légitimité normative. Le discours pratique ne supporte aucune autre influence. Les fondements normatifs qui sont à la base d'un discours proviennent d'un principe a priori, c'est-à-dire de la validité universelle de la parole. Habermas a essayé de défendre ce principe a priori dans la Théorie de l'agir communicationnel (1981) et dans le Discours philosophique de la modernité (1986), où il théorise trois types d'exigences de validité: la science et la technologie, le droit et l'éthique modernes, l'art et la critique artistique modernes. La modernité culturelle n'aurait jusqu'ici fait usage que de la validité universelle de la science et de la technologie. La sociologie de la connaissance scientifique aurait pour tâche de montrer que les processus de modernisation ont été réalisés grâce à une rationalisation fonctionnelle, instrumentale, selon les impératifs du seul intérêt technique. Ce processus aurait pour conséquence la «colonisation de l'existence». Pour décoloniser l'existence, la sociologie de la connaissance scientifique doit «reconstituer ce qui a été réprimé à partir des traces qu'ont laissées dans l'Histoire les épisodes de la communication en butte à la répression».

Par quelles voies pourrions-nous reconstituer ces traces et garantir la transparence discursive, la communication idéale, ainsi que la rationalité et la vérité? La compréhension est un procès très complexe. Il n'y a pas de règle précise pour en assurer la réalisation. L'homme n'est pas doté génétiquement de la capacité de comprendre. Celle-ci se forme tout au long de la socialisation, laquelle nous aide à établir les correspondances entre le monde intérieur et le monde extérieur, entre la subjectivité et l'objectivité, mais ne nous dit pas quand cette correspondance est effectivement réalisée. Dès lors, comment puis-je affirmer qu'un acteur social agit loyalement, conformément aux principes universaux énoncés? De quelle façon puis-je prouver qu'une interaction, à un moment donné, est conforme à la «communicationnalité » discursive.

La seule réponse que donne Habermas à ces questions semble très absconse: il y aurait une raison universelle, une « raison décidée» à vouloir l'avènement de la raison, à prolonger la raison instrumentale par la raison pratique, source de la «communicationnalité » discursive.

Le sociologue reconnaît volontiers que les théories d'Habermas relatives à l'activité communicationnelle valorisent les doctrines de la rationalité et de la vérité. Il admet que ces théories mettent en perspective les sciences et les techniques, sans pour autant les réduire aux savoirs du sens commun. Dès lors, pour une grande majorité de nos contemporains, la science et la technique ne sont plus les moyens indispensables à l'émancipation des hommes. Les savoirs scientifiques ne sont plus la résultante de choix conçus et voulus librement et que, le cas échéant, nous pourrions remettre en question. Ils apparaissent plutôt comme la foucade d'un destin aveugle, imprévisible, irrépressible, piégeant nos civilisations, notre sort commun, accélérant notre déchéance d'êtres naturels et sociaux.

Aujourd'hui, la science et la technique ont perdu leur position de surplomb absolu, elles se trouvent désormais impliquées dans l'existentiel et le social, transformées en partenaires de l'économie, de la politique, et plus généralement de la croissance industrielle et du développement socio-économique. 
Il s'agit là d'un changement capital. Si l'influence de la science et de la technique sur les destinées de l'humanité - sur notre destin matériel naturellement, mais aussi sur notre comportement général, intellectuel, social, et même spirituel - n'est plus foncièrement positive, il faudra alors remanier les fondements mêmes de la science. Il faudra repenser les notions de liberté de recherche, de recherche fondamentale, libre ou orientée, de recherche appliquée (qui vise à trouver la solution d'un problème pratique) et d'études appliquées (qui se concentrent sur l'exploitation effective des connaissances dans l'ordre de la production des biens et des services). Il faudra alors réaliser une véritable opération épistémologique, pour ne pas dire culturelle, afin de distinguer la rationalité scientifique des divers modes de validation idéologique, de la politique, du droit, de l'économie et de la communication médiatique (cf. à ce propos le grand et beau livre de Robert Nozick, The Nature of Rationality, Princeton, N.J., Princeton University Press, 1993).

Mais selon quelles modalités pourrions-nous reconstruire les fondements d'une nouvelle pratique scientifique alors même que la boîte noire contenant les systèmes cognitifs est toujours complètement encodée malgré les efforts de Jurgen Habermas et de tant d'autres? Est-il vraiment suranné de distinguer la recherche de la vérité et la délibération en vue de l'action, la société de pensée et la société de vie, les délibérations pratiques et les examens purement cognitifs? Ne pourrait-on pas emprunter d'autres voies que celle l'artificialisme généralisé qui postule que tout est construit, ou celle du retour à l'empirisme qui récuse la fermeture du sujet sur ses constructions artificielles et réaffirme l'existence d'un monde englobant et dépassant la pensée? Au-delà ou en deçà du constructivisme et du naturalisme, y a-t-il d'autres approches plus attentives à la complexité des interactions entre le savoir et la réalité, entre la finitude du monde physique et social, sa dégradation irréversible, et l'équilibration majorante des nouveaux possibles de Jean Piaget?

L'extraordinaire construction conceptuelle d'Habermas n'aide point le sociologue à répondre à toutes ces questions. Par contre, les théories de l'argumentation ouvrent des perspectives à partir desquelles des réponses plausibles à toutes ces questions pourront être élaborées dans un très proche avenir.

\section{LES SCIENCES SOCIALES SONT-ELLES DES «SCIENCES »?}

Cette interrogation est au cœur des travaux de Jean-Claude Passeron, lequel a condensé ses théories dans Le raisonnement sociologique. L'espace non-poppérien du raisonnement naturel et dans une série d'articles répertoriés dans la bibliographie du volume $\mathrm{Du}$ bon usage de la sociologie. Pavane pour Jean-Claude Passeron, paru en 1996. Mon exposé d'aujourd'hui se base surtout sur l'opus magnum mais tient également compte des discussions avec Antoine Prost (in «Sociétés contemporaines », $\mathrm{n}^{\circ}$ 1, mars 1990, pp. 7-45) et avec Maurice Godelier (in «Raison présente», $\mathrm{n}^{\circ} 108,4 \mathrm{e}$ trimestre 1993, pp. 1-44), ainsi que de l'essai Weber et Pareto: la rencontre de la rationalité dans l'analyse sociologique contenu dans le volume Le modèle et l'enquête. Les usages du principe de rationalité dans les sciences sociales (Paris, Ed. de l'EHESS, 1995), et de l'article 
L'espace mental de l'enquête paru dans le $\mathrm{n}^{\circ} 1$, 1995, et dans le $\mathrm{n}^{\circ} 3,1996$, de la revue «Enquête».

L'ensemble éclaté des travaux et des théories sociologique qui a constitué le corpus sur lequel Passeron a mené une enquête d'épistémologie descriptive sans présupposés durkheimiens l'amène aujourd'hui à une sorte d'auto-critique. En effet, il revient sur les thèses optimistes qu'il défendait naguère avec Pierre Bourdieu et Jean-Claude Chamboredon dans Le métier de sociologue (1968), et admet sans autre que la sociologie a un statut épistémologique singulier par rapport à celui des sciences de la nature. Elle ne satisfait pas au critère poppérien de la réfutabilité (la falsification), elle est dans l'impossibilité de faire de l'expérimentation à la façon des sciences de la nature et pourtant c'est une science, puisqu'elle produit des connaissances et des savoirs auxquels l'épistémologue ne peut refuser le qualificatif d'acquis scientifiques.

Passeron argumente cette thèse générale en se fondant essentiellement sur des descriptions concrètes, c'est-à-dire sur ce que les sociologues font dans le cadre de la recherche empirique.

A la question «Que font les sociologues en réalisant des enquêtes et en formulant dans leurs comptes-rendus ou livres le travail empirique qu'elles impliquent?», Passeron répond ainsi: «Les sciences empiriques sont des langages de description du monde qui se doivent de produire un type particulier de connaissance aux épreuves empiriques que la structure logique de ces langages rend possibles et nécessaires ». En revanche, il faut admettre que les sciences sociales ne disposent d'aucun langage protocolaire unifié de description. La multiplicité de leurs langages de description empêche la formulation d'énoncés de base théoriquement univoques, tandis que les typologies sociologiques, élaborées inductivement (du particulier au général, du fait à la loi, grâce aussi au raisonnement par récurrence ou par induction incomplète), ne permettent jamais d'en dériver des énoncés universels logiquement susceptibles d'être falsifiés. Les typologies sociologiques restent trop liées aux contextes historiques, d'où leur non cumulativité et la multiplicité des constructions théoriques incompatibles entre elles à propos des mêmes phénomènes sociaux. Le monde empirique des sociologues est le monde historique, monde qui, au moins jusqu'ici, s'est avéré rebelle aux théories nomologiques.

Les raisons de cet état de fait sont bien connues et Max Weber y est souvent revenu: les sciences sociales rendent compte de phénomènes singuliers, explicables seulement dans des contextes historiques spécifiques. Dès lors, il est impossible de recréer en laboratoire les phénomènes historiques, mais également d'observer la répétition de formes identiques sous tous les rapports, de régularités, voire d'uniformités.

Passeron critique vertement la thèse de Durkheim qui prétend que le sociologue peut compter sur l'expérimentation indirecte ou sur la quasi-expérimentation. Pour Passeron, nous sommes condamnés à nous «mouvoir et à opérer des compromis argumentatifs entre les exigences logiques du raisonnement expérimental et les exigences descriptives de la contextualisation historique ». Nous sommes constamment soumis a «une tension méthodologique entre [d'une part] la description historique, qui impose la connaissance des configurations réelles comme systèmes singuliers et non reproductibles de cooccurrences de propriétés et [d'autre part] le raisonnement expérimental, qui entraîne toujours à formuler les 
constats de relations fondés sur ces occurrences comme des assertions générales portant sur des corrélations entre variables pures». De plus, dans les sciences sociales, le raisonnement expérimental, même lorsqu'il emprunte ses procédures à l'analyse multivariée, «reste toujours tributaire, (...) de l'interprétation du sens des variations en fonction du contexte»; il ne peut jamais faire l'économie d'une sémantique, soit-elle analytique, structurale, générative, synchronique ou diachronique. Par conséquent, les résultats de l'expérimentation sont ici constamment tributaire d'une interprétation, contrôlée et méthodique, gouvernée par des règles rigoureuses ainsi qu'à la pertinence des questions culturelles posées au départ et affranchies de ce que Passeron appelle «l'illusion herméneutique».

Passeron reprend à son compte l'axiome logiciste suivant lequel nous pouvons définir, par la description, des règles logiques l'équivalence ou la compatibilité entre deux énoncés, mais que nous sommes dans l'impossibilité d'en faire autant entre un énoncé et une réalité empirique observée. Passeron a souvent écrit: «Il n'existe pas de neutralité descriptive du langage par lequel nous parlons du monde, surtout pas du monde historique, même pas quand nous en parlons à travers une sténographie statistique. En aucun cas, nous ne touchons du doigt la réalité telle qu'elle est avant d'être dite: un tableau statistique est irréprochable dans ses énoncés formels puisqu'ils ne disent rien du monde; nous devons, nous, en sociologues dire en prenant les risques interprétatifs d'une langue naturelle ce qu'il nous permet de dire du monde historique, sous contrôle naturel ».

Dans ces conditions, il n'y a pas moyen de fonder la scientificité d'un raisonnement sociologique totalement dépourvu de «langage protocolaire unifié de description du monde historique» sur des critères «naturalistes» puisqu'on ne peut compter que sur les ressources du langage naturel employé dans la théorie. Il n'y a pas de théorie formelle du monde social. Il faut ajouter que Passeron conçoit la scientificité du raisonnement sociologique comme un «va-et-vient» entre la contextualisation historique et le raisonnement expérimental, entre le raisonnement comparatif typologique et l'exemplification, entre l'interprétation soumise à la rigueur des règles statistiques et la «multiplication des constats empiriques dressés dans des sémantiques si non protocolarisées du moins apparentées ». C'est le résultat de ce «va-et-vient» qui produit les concepts sociologiques, semi-noms propres et semi-noms communs, concepts non intégralement définissables par «description définie», au sens de Bertrand Russell.

Passeron conteste la distinction habituelle entre l'histoire comme science des singularités et la sociologie comme science des généralités, entre l'histoire science de l'unique et du singulier et la sociologie science du collectif et du général. La langue de description du monde de l'histoire et de la sociologie est la même. La contrainte du contexte historique ainsi que l'unicité des faits qui singularisent le travail historique jouent le même rôle dans les deux disciplines. Dans les deux cas, l'expérimentation est impossible, d'où l'impossibilité du test empirique de la «réfutabilité » poppérienne. Dans les deux cas, c'est seulement le raisonnement naturel qui permet de construire des contextes typologiquement apparentés et de procéder à des analyses en termes et à l'aide d'exemplification. Dans les deux cas «les affirmations supposent 'l'indexation' sur des contextes dont le sens historique implique lui-même des actes de désignation par rapport à l'espace et au temps, par rapport à une aire géographique ou culturelle, à un règne, à une période». Dans les deux disciplines «la comparaison - et non l'induction - 
est le seul instrument de construction des généralités possibles ». Ici, il n’y a argumentation et preuve qu'au moyen d'une forme spécifique de comparaison, celle qui recourt à «une opération typologique où les contextes dans lesquels sont prélevés les éléments comparés sont des contextes apparentés (mais non substituables) et les concepts guidant la comparaison des concepts analogiques (et non nomologiques)».

Il résulte de ceci que l'histoire et la sociologie sont «indiscernables épistémologiquement». La différence que nous percevons provient uniquement des styles méthodologiques ou argumentatifs en usage dans les deux disciplines, de leurs manières de fabriquer de l'intelligibilité. Il s'agit donc, de différences de pratiques professionnelles, façonnées par l'apprentissage et les habitudes du métier, par le mode de recrutement des chercheurs, par le marché de l'édition et les attentes du public, en somme par la «conformité discursive». En réalité, si les deux disciplines s'expriment à présent différemment, ou autrement, malgré une même structure d'observation, elles le font pour se doter d'une légitimité, pour conquérir une place dans les institutions académiques et pour s'assurer le maintien de leur rôle social.

Peut-on dire que toutes les disciplines sociales relèvent d'un tel statut, alors que l'expérience montre que certaines d'entre elles, par exemple l'économie, la démographie ou la linguistique, sont arrivées à s'autonomiser, à construire des modèles comme si elles étaient des sciences nomologiques, à se donner la possibilité d'abstraire certaines relations d'un ensemble d'interdépendances et être ainsi en mesure de construire un objet scientifique propre, soit-il partiel?

Pour Passeron «une science autonomisante court après un leurre quand elle croit épuiser l'historicité culturelle des phénomènes à force d'ajouter des 'variables exogènes' à un modèle qui repose sur l'autonomisation. La force explicative des modèles, c'est leur capacité d'autonomiser des dimensions mais en oubliant momentanément le caractère inépuisable des contextes dans lesquels fonctionnent les interactions sociales ou les continuités historiques ». Or, lorsque ces sciences veulent résoudre des vrais problèmes, elles doivent revenir sur le déroulement historique de leurs phénomènes les plus spécifiques et recourir «à des propositions qui ne sont, elles, que 'numériquement universelles', c'est-à-dire à des propositions dont la généralité est indissociable de la particularisation du sens par des 'déictiques'», c'est-à-dire par le recours à ce qui sert à montrer, à désigner un objet singulier, déterminé, dans une situation spécifique.

Dans ces conditions, la conclusion de Passeron devient inévitable: les seuls modèles utilisables dans les sciences sociales sont des modèles interprétatifs, des modèles déictiques. «Et seule la rigueur de la méthode dont on se sert pour construire l'interprétation décide de leur caractère scientifique ou non». On a toujours affaire à plusieurs théories interprétatives, à des reconstructions partielles, en concurrence entre elles, mais non pour se réfuter les unes les autres. Pour cette raison, elles servent toutes au travail empirique et au travail d'enquête, à des intelligibilités sectorielles du monde historique.

Je peux admettre que toute théorie sociale est une interprétation, que les modèles interprétatifs sont nombreux, mais pas que toutes les interprétations aient la même valeur. Ici, il ne s'agit pas de choisir entre des théories rivales, il s'agit plutôt de savoir laquelle parmi ces théories possède les capacités interprétatives les plus puissantes, ou les plus générales. De quelle façon pouvons-nous répondre à une telle question? 
En outre, lorsqu'on affirme que ni l'herméneutique, ni la nomologie, ni le naturalisme, n'ont de place dans les sciences sociales, ne risque-t-on d'attribuer à ces dernières une sorte d'intelligibilité de sens commun dont le contrôle est difficilement codifiable?

A cette dernière objection, que Gilles-Gaston Granger a formulée le premier, Passeron répond que l'intelligibilité comparative dans les sciences historiques est à l'opposé de l'intelligibilité du sens commun. Il invoque Max Weber pour appuyer ses arguments: «... la sociologie me semble devenue tout entière weberienne, pour autant, du moins, quelle produit une intelligibilité qui lui appartient en propre. La sociologie vivante est weberienne ... parce qu'elle utilise directement les typologies ... indirectement, parce qu'[elle] n'abdique ni l'explication, ni la compréhension». Selon Passeron, le sociologue allemand associe toujours explication et interprétation et quels que soient sa méthode et son objet, il reste indépassable pour dire, sans faux-fuyant ni auto-dévalorisation scientifique, ce que signifie «être faux ou vrai » dans une recherche, dans un discours assertorique de science sociale. Et pour mieux faire comprendre la méthodologie weberienne du langage idéal-typique, Passeron la traduit dans la description logique et sémiologique des langues naturelles («nom propre» et «nom commun»). Il est convaincu que «le sens complet d'une argumentation historique se formule dans des énoncés qui sont indissociables de l'énonciation en langue naturelle». (J.-Cl. Passeron, L'espace weberien du raisonnement comparatif, in M. Weber, Sociologie des religions. Textes réunis, traduits et présentés par J.-P. Grossein. Introduction de J.-Cl. Passeron, Paris, Gallimard, 1996, pp. 1-49).

Cette réponse est recevable dans la mesure où l'intelligibilité comparative serait formalisée et contrôlable. Mais l'est-elle dans la pratique quotidienne du travail sociologique?

Certes, la sociologie est historique en sa pertinence historique dernière. «Dès qu'une analyse sociologique entend fonder ses généralités sur l'observation du cours du monde historique, toujours 'singulier', elle exprime, en effet, qu'elle le sache ou non, ses énoncés dans un langage dont les assertions - tant par le statut logique de leurs concepts que par l'argumentation des conclusions auxquelles elles conduisent - ne doivent leur généralité conceptuelle qu'à leur forme idéaltypique.» (Ibid., p. 12). Passeron essaie d'approfondir cette question par le biais d'une description factuelle du travail du sociologue. Comment advient la transformation de l'information sur le monde historique dans les sciences sociales? L'analyse est exposée dans l'article intitulé L'espace mental de l'enquête, cité cidessus.

Selon les propres dires de Passeron, cet article nous détaille les démarches argumentatives qui, dans une enquête sociologique, donnent, implicitement et explicitement, leur sens aux opérations statistiques d'une méthodologie quantitative (recueil des données, codage, traitement, analyse et synthèse des résultats). Après avoir analysé le recueil des informations de base, puis les transformations qu'elles subissent tout au long de la recherche, Passeron décrit comment le sociologue construit les preuves et les interprétations pour édifier le raisonnement sociologique. Ce travail se fait dans un «espace logique»-expression empruntée à Wittgenstein - déterminé comme l'«ensemble des contraintes qui définissent pour une assertion le sens de ce que signifie pour elle le fait d'être vraie ou fausse», ainsi que dans un espace sémantique caractérisé ainsi: «Ensemble des 
champs sémantiques des concepts qu'il faut prendre en compte pour que le sens assertorique des propositions qui le contiennent ou en dépendent soit complètement défini ».

Si dans les disciplines formelles «l'espace assertorique» des propositions se réduit à son espace logique puisqu'il est, en principe, entièrement défini dans une langue artificielle par les axiomes et par les définitions-transformations des opérations logiques, dans les disciplines sociales, les observations et les opérations de traitement des faits appartenant à des contextes singuliers sont inséparables d'argumentations produites en langue naturelle. En d'autres termes, tous les actes techniques qui donnent un sens aux différentes phases d'une enquête quantitative dépendent sémantiquement les uns des autres dans l'espace argumentatif des preuves. Il faut ajouter que cette espace n'est jamais fermé étant donné que les langues naturelles restent toujours ouvertes à une transformation du langage de la description, d'où l'obligation, «pour engendrer des connaissances et éviter la répétition ou la démultiplication pure et simple des descriptions, de poser sans cesse de nouvelles questions interprétatives au monde, ce qui revient à remettre en mouvement et en question les articulations de son 'univers du discours'».

Passeron étudie les implications sémantiques et la signification argumentative des opérations pour recueillir les informations, pour les coder, les traiter, en faire la synthèse et la transmettre grâce à l'écriture sociologique. La différence entre le raisonnement du sociologue et celui du statisticien est bien relevée. Alors que le statisticien ne fait pas intervenir dans ses calculs le sens empirique de ses assertions sur le monde, le sociologue est guidé par l'interprétation du sens culturel des actions soumises à la mesure. Il fait une sur-interprétation contrôlée, ce qui l'expose parfois à des interprétations imprudentes ou inadéquates.

Passeron reconnaît qu'une telle situation est périlleuse et inconfortable, pleine de risques et d'incertitudes: «En l'absence du contrôle formel» que permet «la langue artificielle d'un symbolisme» ou d'un «contrôle qu'exercerait la valeur estampillée des mots déjà contrôlés par un paradigme» au sens de Kuhn, «le chercheur est son propre contrôleur, avec parfois l'aide du lecteur. D'où, on le voit tous les jours, de terribles relâchements de surveillance, de la part de l'un comme de l'autre».

Mais puis-je être mon propre contrôleur? Je suis incapable de donner une réponse convaincante. J'aimerais rappeler qu'il y au moins trois manières de comprendre une situation de recherche-enquête: celle qui se déroule au présent quand on s'y trouve engagé comme acteur (le Touraine de Lutte étudiante ou le Bourdieu de La misère du monde); celle où on se trouve plongé passivement, voire comme témoin neutre (le Boltanski de La souffrance à distance). Enfin, il y a l'enquête qui a eu lieu et qui est devenue un objet de réflexion (le Boudon de L'inégalité des chances ou encore presque tous les travaux de sociologie historique de Norbert Elias). Parfois ces trois niveaux se chevauchent et leur recomposition est impraticable. Peut-on dire que cela est possible des années plus tard, lorsque l'historien de la sociologie aura la distance que les contemporains n'ont jamais eu à l'endroit, par exemple, de Georg Simmel?

Hans Robert Jauss (1921-1997), critique littéraire allemand, a montré dans Pour une esthétique de la réception (Paris, Gallimard, 1973) que c'est la réception «d'une œuvre» par le public qui lui confère son sens, soumis dès lors aux variations du temps. Le même auteur dans Wege des Verstehens (Munich, Fink, 1995) a montré qu'on ne peut arriver à la compréhension ni par la force, ni par la régle- 
mentation, ni même par une argumentation logique. Il affirme que comprendre quelque chose ou quelqu'un, implique toujours un assentiment. Dès lors, il m'est impossible de comprendre ce que je n'approuve pas. Si comprendre signifie pardonner, trouver des raisons suffisantes à n'importe quelle action sociale, alors pourquoi travailler sur la criminalité, sur les massacres ethniques, sur le racisme et ainsi de suite? Pourquoi recenser et étudier les faits, si comprendre postule l'assentiment et la compréhension qui à leur tour impliquent l'approbation?

Existe-t-il un moyen d'échapper à ces dilemmes et à ces paradoxes? Est-il possible d'établir le vrai et le faux, d'aménager un espace où la vérité sociologique prendrait un sens spécifique?

Passeron a essayé de saisir la complexité de cette problématique dans Le temps donné aux tableaux. Compte rendu d'une enquête au Musée Granet (Marseille, Documents de Cercom/Imerc, décembre 1991), où il étudie de quelle façon les réinterprétations du sens et du prix accordés aux œuvres par leurs audiences successives ont ouvert le chemin à la théorie de la réception de Jauss. Cependant, les bonnes réponses à ces questions ne peuvent se trouver que dans une théorie de la rationalité, dans la reconstruction rationnelle de ce que Pareto appelait le nonlogique, c'est-à-dire les éléments affectifs, mécaniques, singuliers, normatifs ou inconstants des actions humaines. Passeron affirme haut et fort qu' «En toute science sociale la connaissance scientifique ne peut être que rationnelle, indépendamment du quantum ou de la forme de rationalité que la démarche scientifique postule dans les objets qu'elle construit... Une hypothèse de rationalité, qui postule la cohérence de choix guidés par la raison, est en tout état de cause requise dès que l'on prend les actions humaines pour objet d'étude en acceptant aussi bien l'hypothèse d'une liberté de choix qu'un principe causaliste d'explication ». Toutefois il fait suivre cette affirmation d'une importante réserve: «... dès lors, si les individus sont rationnels alors leurs choix pourront, en principe, être expliqués par des observateurs partageant avec eux cette rationalité ». Et sinon? La réponse des sociologues classiques (Pareto, Weber) et celles des théories économiques pures n'est plus la même. Passeron a très perspicacement noté (La rationalité et les types de l'action sociale chez Max Weber, in «Revue européenne des sciences sociales », XXXII, 1994, n. 98, pp. 5-44) que Pareto et Weber ont refusé à la fois le rien et le tout: «Ils n'ont voulu ni congédier le principe de rationalité comme principe organisateur de l'enquête sociologique sur les configurations et les déroulements historiques, ni transformer cet opérateur méthodologiquement privilégié du questionnement des actions sociales en principe d'enfermement théorique».

Je crois que cette thèse mérite une discussion approfondie, car elle est au cœur des débats actuels sur la connaissance et d'elle dépend la résolution de la controverse opposant la sociologie de la connaissance scientifique s'inspirant des «classiques » à la sociologie et à l'anthropologie du «programme fort». Il faut donc lui consacrer un peu de notre attention et de notre temps.

\section{DE LA RATIONALITÉ}

Clarifions tout d'abord les mots du vocabulaire que nous allons utiliser pour parler de la rationalité. 
Commençons par le terme Raison (lat. Ratio, all. Vernunft). Dans le langage quotidien celui-ci signifie jugement, pensée, discernement, il évoque une connaissance opposée à ce qui vient de la foi, ce qui nous permet de connaître, de juger et d'agir d'après des principes. Est réputé Raisonnable celui qui pense, raisonne, agit selon les principes de la raison, avec bon sens, d'une manière convenable et mesurée, tandis que le Raisonnement constitue l'activité, l'exercice de la raison.

Les acceptions techniques de ces mots sont plus complexes et nombreuses. En effet, en épistémologie la raison est la faculté générale propre aux hommes d'enchaîner des propositions pour faire des déductions, des démonstrations, des assomptions, des relations, etc. La Raison y est définie également comme la capacité d'obtenir une connaissance, de produire un savoir grâce à un procédé programmé préalablement, ou comme le guide souverain dans le domaine de la recherche et dans le travail d'enquête. On appelle aussi raison le fondement, l'essence, la forme, la substance, la raison d'être d'une chose ou d'une élaboration ainsi que l'argument utilisé ou la preuve apportée, ou encore la façon de dire le vrai. La Rationalisation, par contre, est la faculté de penser rationnellement et de dévoiler la raison qui fait comprendre ou qui explique une action, un discours ou un comportement.

Avec le mot rationalisation on désigne le processus par lequel les sciences se constituent en disciplines théorétiques grâce à la méthode axiomatique, au langage logico-mathématique, ou à d'autres procédures formelles. Parfois, des sociologues utilisent le mot rationalisation pour décrire les tentatives d'un sujet visant à justifier et faire valoir, grâce à des discours apparemment logiques ou plausibles, des croyances fondées sur des émotions, des instincts, des préjugés, des habitudes, des intérêts, etc. Les sociologues, notamment ceux qui étudient les organisations, appellent aussi rationalisation l'opération qui consiste à adapter, à partir d'une étude préalable, les moyens disponibles aux objectifs poursuivis. Le Raisonnement est alors l'opération par laquelle on passe, par démonstration, des prémisses à une conclusion. Il s'agit d'une procédure d'inférence ou de preuve. Le terme est utilisé pour indiquer la manière dont s'exerce l'activité de la raison.

Depuis l'Antiquité, on distingue le raisonnement inductif, du raisonnement déductif et du raisonnement analogique. Dans la terminologie contemporaine, on préfère parler de raisonnements explicatifs, analytiques ou déductifs, et de raisonnements amplifiants, synthétiques ou inductifs.

C'est grâce à Charles Sanders Peirce (1839-1914), un des pionniers de la philosophie de la science contemporaine, que nous avons aujourd'hui coutume de distinguer les trois étapes de la méthode hypothético-déductive: l'abduction (la procédure d'acceptation d'une hypothèse explicative), la déduction (la dérivation des conséquences vérifiables par l'hypothèse explicative) et l'évaluation de l'hypothèse à la lumière des conséquences vérifiées, dite aussi induction qualitative. Pour les tenants de cette doctrine (le pragmatisme), toute croyance est fonction d'autres croyances acceptées préalablement, d'où l'affirmation que les conclusions scientifiques sont toujours expérimentales, que la science peut déceler et corriger ses propres erreurs, que la rationalité de la méthode scientifique ne dépend pas de la certitude de ses conclusions, mais de sa capacité d'autocorrection, cette dernière capacité étant toujours soumise à la surveillance illimitée de la communauté universelle des chercheurs et des savants, d'aujourd'hui et de demain. 
Puisqu'il n'y a pas en sociologie de langage unifié, les chercheurs en sciences sociales utilisent tous ces mots de façon polyvalente, d'où la constante nécessité d'en établir, à partir du contexte, l'exacte sémantique, d'en cerner précisément les connotations et les dénotations.

Le concept, véritablement stratégique dans notre discipline, de Rationalité nous en offre un exemple emblématique. Ses définitions et les utilisations que nous en faisons, sont innombrables. Un gros livre ne suffirait pas à les passer toutes en revue. Dans le langage commun, la rationalité est le caractère de ce qui est raisonnable, qui se conduit selon raison. Dans le langage scientifique, par contre, la rationalité signifiait à l'origine ce qui était fondé sur la raison et pouvait donc être considéré comme intelligible. En d'autres termes, c'était ce qui obéit aux lois de la raison, qui peut être connu et expliqué par la raison. Puis, par extensions successives, la rationalité est devenue ce qui est fondé sur un calcul et qui cherche à rendre compatible des moyens et des fins. Puisqu'il existe une grande diversité de fins, il s'en suit qu'il y a différents types de rationalité. On n'a plus affaire à la rationalité mais à des rationalités. Les connaissons-nous toutes ? Sontelles différentes les unes des autres, ou bien constituent-elles des cas particuliers d'une seule théorie générale?

Les pères fondateurs de la sociologie parlaient déjà de rationalité lorsque l'enquêteur observait une adéquation objective entre les moyens adoptés et les fins poursuivies. L'histoire de l'élaboration, par Weber et Pareto, du concept de rationalité dans l'analyse sociologique et celle de toutes ses reformulations successives a été faite, de façon magistrale, dans l'essai de Jean-Claude Passeron publié dans le volume déjà cité Le modèle et l'enquête. Les usages du principe de rationalité dans les sciences sociales, ainsi que dans diverses études de Raymond Boudon.

Dans l'introduction à l'ouvrage Le modèle et l' enquête, Passeron explique très clairement un point souvent oublié ou négligé par les chercheurs. «L'adjectif 'rationnel' ne peut signifier que 'justifié' (pour une fin) ou 'approprié' (pour un moyen). Le terme comporte, si l'on veut définir les rapports des fins entre elles ou entre des moyens et des fins, une exigence de cohérence logique; il implique aussi la stabilité de cette cohérence dans le temps, c'est-à-dire la constance des règles du raisonnement ou du calcul des décisions. La description de la rationalité d'une action suppose ainsi toujours la description d'un raisonnement, actuel ou virtuel, puisque ce n'est qu'à un raisonnement - c'est-à-dire à un système de relations entre des termes ou des propositions - que les propriétés de cohérence et de constance peuvent s'appliquer».

Qu'en est-il des raisonnements auxquels font défaut l'une ou l'autre, voire ces deux propriétés logiques? Sont-ils irrationnels? La réponse des sociologues est négative. Le fait que la structure d'une action révèle l'absence de raisonnement ou l'existence de raisonnements incomplets, passionnels ou incohérents, arbitraires, ne doit pas nous empêcher de rechercher «une nécessité explicative - une intelligibilité reconstruite - en appliquant à de telles structures d'action les méthodes de description, d'enquête et d'analyse qui découlent de la rationalité comparative propre aux sciences historiques ou cliniques».

Mais comment élaborer, formuler, en somme fabriquer cette «intelligibilité reconstruite » dont parle Passeron?

Le maniement de la théorie de la rationalité mise au point par les économistes néo-classiques (rechercher le maximum de satisfaction au moindre coût) n'est pas 
facile, lorsqu'on doit rendre compte de la complexité du monde réel. Qu'il s'agisse de l'appréciation de ce maximum pour un individu (rationalité individuelle), pour un groupe ou pour une société tout entière (rationalité collective), l'application de la théorie pose des problèmes difficultueux et pointilleux. Il faudrait maîtriser totalement la situation, disposer d'une vision globale, avoir des informations précises et complètes, pour rechercher le maximum de satisfaction au moindre coût. Existe-t-il une situation dans laquelle l'acteur, disposant de toutes les informations et ayant éliminé toutes les incertitudes, les aléas, les précarités, puisse agir en fonction de son intérêt et en connaissance de cause?

Et comment doit-on définir l'action? Est-ce un processus de transformation de la réalité, ou une manipulation de cette même réalité pour satisfaire un intérêt préalablement identifié et déterminé au sein d'un réel préfixé?

Quelques chercheurs ont tendance à choisir cette dernière intellection, soit l'optimum collectif préfixé de l'extérieur, voire la détermination des meilleures solutions possibles à tous les problèmes. Mais pour être appliquée, une telle théorie doit utiliser des hypothèses très limitatives et distinguer diverses sortes d'optima (optimum dit d'ordre inférieur, optimum avec contraintes institutionnelles, et ainsi de suite). Doit-on apprécier l'action à partir de ses conséquences, ou des principes qui l'inspirent? Max Weber était de l'avis que, dans certaines situations, il est préférable d'analyser les principes plutôt que les conséquences, d'où sa célèbre distinction entre la rationalité instrumentale et la rationalité axiologique, entre la rationalité à court terme et rationalité à long terme. Ceci dit, sur quelle base, à partir de quels critères choisit-on les principes plutôt que les conséquences, ou vice-versa? Comment distinguer le court terme du long terme?

Tout cela n'est ni aisé, ni par ailleurs toujours possible. Pour cette raison, une telle théorie n'arrive que rarement à rendre compte de la complexité du réel. Elle n'arrive pas non plus à nous donner les moyens, ni même à nous indiquer comment et pourquoi, par exemple, les actions de Sadam Hussein ou de Bill Clinton pourraient être expliquées grâce à la généralisation suivante: «Lorsque la satisfaction de chacun des membres du groupe ou de la société peut être augmentée sans que celle d'un autre membre ne soit diminuée, tout comportement visant à accroître cette satisfaction est dit conforme au principe de rationalité collective».

A côté de cette rationalité procédurale où un individu rationnel calcule convenablement et correctement, on essaie de définir une rationalité substantive, mais incomplète, dite limitée, qui gouverne le comportement de l'individu dans un monde incertain et imparfaitement connu. Les modèles de rationalité limitée sont nombreux, ils ont des coûts spécifiques, mais ils ne sont pas soumis aux exigences des règles de la rationalité instrumentale. En effet, la rationalité substantive ou limitée est englobée par la dimension cognitive des agents sociaux, en d'autres termes par les conceptions et les représentations du monde, délimitant, coordonnant et garantissant leurs actions et décisions dans le monde réel.

Ainsi formulé, le principe d'une rationalité unique reste insaisissable, mieux, il se révèle impuissant dans les tentatives d'explication de la variété et de la complexité du réel. Pour cette raison, le prix Nobel Herbert H. Simon (Reason in Human Affairs, Stanford, Cal., Stanford University Press, 1983) a proposé d'abandonner la notion de maximisation au profit de celle de satisfaction, de partir du constat que l'acteur n'est ni omniscient ni infaillible, qu'il dispose de rensei- 
gnements insuffisants, imparfaits, même défectueux, et pourtant qu'il doit agir. Sur ce point, je n'ai pas besoin de vous en dire davantage car le regretté Ernest Gellner a écrit en la matière un livre (Reason and Culture. The Historic Role of Rationality and Rationalism, Oxford, Blackwell, 1992) délicieux et précieux, très érudit et néanmoins passionnant, et que je vous conseille de lire attentivement.

D'après Herbert H. Simon, l'acteur social est incapable de maximiser ses intérêts, il ne peut pas obtenir les résultats les meilleurs, car il ne dispose presque jamais de toutes les informations indispensables et ne peut maîtriser les incertitudes du contexte d'action. Il ne lui reste donc qu'à rechercher une satisfaction, le bien connu «satisfecing», c'est-à-dire des résultats plus au moins satisfaisants. L'exemple classique nous est donné par le joueur d'échecs, constamment confronté à des incertitudes considérables et obligé d'agir en situation de rationalité limitée. Il doit se représenter les coups plausibles et probables de son adversaire, faire des calculs prévisionnels en situation d'incertitude, agir, puis corriger ses calculs en fonction des réponses effectives obtenues. Mais pour agir de la sorte, le joueur d'échecs doit faire appel à toutes ses ressources cognitives, aux informations disponibles sur les jeux de l'adversaire, à des représentations plausibles des réactions et des attentes de celui-là, en bref à des raisons réputées comme les plus solides dans la situation d'interaction du moment précis.

Raymond Boudon fait, dans un pré-print diffusé à la fin du mois de mars 1996 et intitulé Au-delà de la rationalité limitée?, une remarque très pertinente et perspicace. Il affirme: «Lorsqu'on analyse les croyances scientifiques, on part généralement du principe que ceux qui les endossent ont des raisons fortes de le faire; la décision d'opter pour une théorie scientifique plutôt que pour une autre est déterminée par des raisons et ces raisons sont perçues par l'acteur, en l'occurrence le scientifique, comme fortes. Ainsi, lorsque Joseph Priestley (1733-1804) croit en l'existence du phlogistique, il a le sentiment que sa croyance est fondée sur des raisons fortes. Raisons fortes ne veut pas dire raisons vraies (plus personne ne croit aujourd'hui à la théorie du phlogistique). Mais 'raisons fortes non vraies' ne veut pas dire non plus 'raisons liées à des idiosyncrasies personnelles'. A l'évidence, Priestley n'était pas le seul à croire au phlogistique. C'était au contraire une croyance répandue. Et elle était répandue parce que, étant donné l'état du savoir, les arguments de Priestley représentaient des arguments forts. Ils le restèrent jusqu'à ce que les accumulations d'autres considérations, qui n'étaient pas encore connues à l'époque, affaiblissent irréversiblement les arguments de Priestley au profit de ceux de A.L. de Lavoisier (1743-1794). Les décisions de Priestley (endosser la théorie du phlogistique) et de Lavoisier (ne pas l'endosser) sont contradictoires entre elles; l'avenir devait démontrer que l'une des croyances était fausse et l'autre vraie; mais dans le contexte dans lequel la 'décision' de Priestley et de Lavoisier est prise, l'une est aussi rationnelle que l'autre».

Pour Boudon, si une croyance fait sens, si elle offre des raisons fortes, elle est endossée, «étant entendu que des raisons qui sont fortes aujourd'hui peuvent cesser de l'être demain (si le contexte cognitif se modifie), et que ce qui est vrai ici ne l'est pas forcément là (si le contexte cognitif n'est pas le même ici et là). L'histoire de la science fait donc clairement apparaître comme banale la situation où s'installe dans les milieux scientifiques la croyance que ' $X$ est vrai' parce qu'il existe des raisons fortes, bien que fausses, fragiles ou douteuses d'y croire, étant donné l'état de la science hic et nunc». 
Dans cette perspective, la rationalité ne se réduit pas à la rationalité instrumentale; le sujet théorise le problème à résoudre, puis endosse les raisons qui lui paraissent les plus fortes et les plus avantageuses. Le savant procède ainsi, quitte à les corriger, à les modifier ou à les abandonner.

Boudon insiste sur le fait qu'il faut découvrir le système de raisons qui est à la base d'un choix, d'une action ou d'un comportement et qu'il faut analyser le fondement argumentatif de ce système de raisons, car c'est le seul moyen d'éviter d'avoir affaire aux apories du conventionnalisme et du relativisme. C'est la seule approche analytique qui nous permette de «comprendre pourquoi l'on croit à telle valeur dans tel contexte et d'échapper à l'attitude défaitiste qui consiste à postuler l'indétermination des fins» (Sens et Raisons: Théorie de l'argumentation et sciences humaines, in «Hermès», 1995, n. 16, pp. 29-43).

Des critiques fouillées ont été faites au rationalisme méthodologique de Boudon. Certains affirment qu'il ne s'agit que d'une méthode descriptive, soucieuse de montrer la manière selon laquelle il faut expliquer les phénomènes. Son champ d'application serait trop étroit, puisque seuls les comportements et leurs conséquences sont pris en charge par l'analyste. Il s'agirait d'un modèle très simplifié qui ne peut s'appliquer qu'à un nombre limité et particulier de phénomènes, là où il est possible d'étudier l'action causale au sens large des forces non causales au sens étroit. Il s'agirait de cas d'espèces non susceptibles de rendre compte de l'infinie complexité du monde réel.

Ces critiques ont été développées par divers auteurs, notamment par Philippe Van Parijs (Le modèle économique et ses rivaux. Introduction à la pratique de l'épistémologie des sciences sociales, Genève, Droz, 1990) et, plus récemment, par le chercheur genevois Pierre Moessinger (Irrationalité individuelle et ordre social, Genève, Droz, 1996, et Les raisons et les sentiments, in «L'Année sociologique», 1996, 46, n. 1, pp. 213-223). Je ne peux que vous renvoyer à la lecture de leurs travaux. Pourtant, je persiste à croire que l'entreprise de Boudon visant à démontrer que toutes ces formulations de la rationalité ne sont pas contradictoires entre elles, qu'elles constituent des variations sur le même thème, mérite d'être approfondie. De fait, elle commence à l'être, y compris sur la base d'autres options méthodologiques, comme semble le prouver une série de publications, parmi lesquelles je vous signale Les formes de l'action, publié sous la direction de Patrick Pharo et Louis Quéré (Paris, Ed. de l'EHESS, 1990) et La théorie de l'action. Le sujet pratique en débat (Paris, CNRS Editions, 1993) et bien entendu le volume déjà cité Le modèle et l' enquête.

Vous savez que Jean-Claude Passeron doute du fait qu'il soit possible d'élaborer une théorie étendue de la rationalité en faisant l'économie des notions de jugement et d'autonomie. Et d'après lui, une théorie incluant ces deux dernières notions risque de devenir une théorie du vrai et du bon, donc une théorie de l'éthique, de l'équilibre social et de la justice.

Je ne partage pas entièrement ce point de vue. La sociologie de la connaissance scientifique perdrait sa raison d'être sans une théorie de la rationalité. Qu'il y ait plusieurs rationalités, que certaines d'entre elles s'éloignent des modèles disponibles, ne doit pas nous empêcher de les analyser et de tenter de les coordonner, même si cela reste difficile dans le contexte cognitif actuel. Je crois que les difficultés dérivent du fait que nous incluons dans la doctrine de la rationalité deux concepts qu'il faut maintenir distincts: la raison et le raisonnement. 
La raison a une histoire, c'est elle qui crée nos objets d'étude, nos constructions symboliques; le raisonnement est une compétence et une compétence qui s'acquiert, qui évolue et se transforme en évoluant. Cette distinction nous place, certes, devant une difficulté pour laquelle aucune solution n'a été trouvée jusqu'ici. Nous ne savons pas saisir les objets et les constructions de la raison sans utiliser des raisonnements, des compétences pour les mettre en évidence. Le problème est difficile et nous l'avons dit au cours du colloque consacré à Raison et relativité des valeurs, dont les actes ont été publiés en 1987 («Revue européenne des sciences sociales», XXV, 1987, n. 74). Mais nous ne devons pas nous laisser gruger. Puisque la vérité ultime ne se trouve dans aucun livre, nous ne pouvons établir la vérité qu'après coup, après bien des doutes, des débats, des travaux, des erreurs; après des confrontations, des critiques et des contrôles. Faire de la science, c'est critiquer les raisonnements des autres, tout en produisant les siens. D'où l'obligation de connaître le mode de production de ces raisonnements, de se souvenir régulièrement que la science est argumentation et communication dans un cadre social donné, ainsi que Jean-Blaise Grize vient de nous le rappeler dans son dernier et passionnant ouvrage Logique naturelle et communications (Paris, PUF, 1996), dont je vous ai déjà parlé.

Qu'il soit indispensable de distinguer raison et raisonnement pour saisir le rôle de la temporalité dans le travail scientifique - tout comme pour comprendre les variations dans l'acceptation ou le rejet d'un savoir par les communautés savantes - peut être exemplifié en suivant l'histoire du géologue français Jacques Deprat telle qu'elle nous a été rapportée par M. Durand-Delga (L'affaire Deprat, in «Travaux du Comité Français d'Histoire de la Géologie», n.s., 1990, n. 4, pp. 117-212, et L'affaire Deprat, l'honneur retrouvé d'un géologue, in «La Recherche», vol. 237, novembre 1991, pp. 1342-1346).

Au mois de juin 1919, un jury d'honneur déclarait que le géologue Deprat avait placé des fossiles européens dans la collection de fossiles asiatiques ramassés en Chine et au Viêt-nam. Sur la base des théories alors courantes sur l'âge des fossiles, le jury affirmait que les trilobites étaient européens. Les connaissances de l'époque ne permettaient pas de rendre compte convenablement de leur présence en Asie.

Les protestations d'innocence et d'intégrité de Deprat n'émurent pas le jury d'honneur du Collège de France. Le géologue fut licencié et expulsé de la Société française de Géologie. Dès lors, l'enseignement et la recherche lui furent interdits. Pour vivre, il dut se consacrer à des travaux littéraires et journalistiques sous le nom d'auteur de Herbert Wild.

Depuis un peu plus d'une dizaine d'années, on s'est aperçu que différents géologues avaient trouvé en Asie un grand nombre de trilobites de la même espèce que ceux récoltés par Deprat entre 1916 et 1919. De plus, la théorie des plaques tectoniques fournissait l'explication d'une telle présence: durant environ un demi milliard d'années, l'Europe méridionale et l'Asie du Sud-Est étaient proches, d'où la similarité des fossiles.

Le 10 juin 1991, la Société Française de Géologie, réunie en assemblée plénière, a fait amende honorable, a effacé la condamnation et a réintégré dans ses rangs, à titre posthume, Deprat.

Cet exemple et bien d'autres que je pourrais vous citer montrent que les argumentations sont redevables du savoir d'une époque, qu'elles sont le produit d'un 
contexte historique, d'une certaine structure de la connaissance, mais qu'elles ne touchent pas la nature même de la Raison, le projet ultime de la Science. Il est indispensable, donc, de distinguer la Raison du Raisonnement ainsi que nous avons essayé de le faire dans le volume Raison et relativité des valeurs ( Revue européenne des sciences sociales », XXV, 1987, n. 74, pp. 161-194).

\section{UNE CONCLUSION PROVISOIRE}

Il est temps de tirer une conclusion provisoire et un peu personnelle de ce que nous avons étudié et débattu pendant cette année, une conclusion, en vérité, plus culturelle au sens large que sociologique au sens strict.

Afin de connaître, comprendre et interpréter les phénomènes, naturels ou sociaux, afin d'en dévoiler les structures profondes, les hommes ont si fortement misé sur les apports de la recherche scientifique et des innovations technologiques que ces dernières sont devenues essentielles au fonctionnement et à la survie des Sociétés. Or celles-ci, pour être sûres d'obtenir une production stable et continue, ont dû créer, dès le XVII ${ }^{e}$ siècle, des institutions ad hoc. Elles ont dû les doter de pouvoirs d'investigation et d'initiative très larges et autonomes, de compétences et de privilèges importants ainsi que de moyens matériels (laboratoires et appareils) et culturels (bibliothèques, congrès, revues, etc.) de plus en plus considérables.

Bien que le rôle de la science et des techniques dans la production des connaissances, dans le développement économique et social, dans l'amélioration des conditions de vie des populations soit incontestable; bien que la capacité de la science à ne jamais s'incliner devant les mystères du réel et à tout soumettre à l'épreuve de l'expérience soit entièrement prouvée, les critiques contre sa prétention à être une valeur absolue et à constituer la référence ultime pour tous les systèmes normatifs se sont multipliées et radicalisées de façon exponentielle. Pour certains, la science n'est désormais que la croyance occidentale de maîtriser rationnellement tous les phénomènes, naturels et sociaux, à l'aide du calcul, de la formalisation et de l'informatique. Cette croyance constituerait, avec l'économie, l'idéologie de la Modernité. Son but serait d'assurer la prééminence hégémonique d'une forme d'organisation sociale sur toutes les autres de la Planète.

La critique de la science, sa réduction à une croyance sociale pareille à n'importe quelle autre croyance sociale, ont mis en péril un certain nombre de préconstruits culturels et surtout les notions d'objectivité et de vérité. Ce réductionnisme a aussi alimenté un relativisme généralisé selon lequel il y a autant de vérités, de valeurs et de points de vue que de groupes sociaux ou de communautés d'intérêts, de cultures diverses, de conceptions du monde. Les universaux de la science ne seraient que les cache-misère de l'idéologie de la Modernité.

Dans les débats actuels sur la science, les tenants du «programme fort» se sont tout particulièrement distingués en défendant ce point de vue. Un de ses partisans, Bruno Latour, a eu le mérite de synthétiser une telle position en des formules lapidaires, par exemple dans son livre Nous n'avons jamais été modernes et plus récemment dans l'article La modernité est terminée (in «Le Monde», 29 août 1996, p. 11), d'où je tire les citations que voici: «Bien que l'idée de progrès ait été efficace, qu'elle ait servi pour choisir certaines combinaisons de facteurs, pour 
accélérer certains choix techniques ou économiques, elle ne saurait décrire ce qui s'est passé en Europe depuis trois siècles. Les sciences, les techniques, les marchés, n'ont jamais eu l'aspect lisse, objectif, progressif, inhumain que les Européens ont souhaité leur donner afin de construire leur idée de progrès. Au lieu de nous arracher à un passé archaïque, les sciences et les techniques nous ont au contraire plongé, toujours davantage, dans une riche matrice anthropologique... Derrière nous peut-être, dans le passé, nous confondions les faits et les valeurs, les sciences et les politiques, mais devant nous, à coup sûr, le nœud qui relie les faits, ce que sont les choses et les valeurs, ce que veulent les humains, se trouvera plus serré encore, plus indémêlable... Nous n'avons jamais été modernes... Nous n'avons jamais avancé vers un surcroît d'efficacité et de rentabilité qui nous éloignerait toujours davantage d'un passé archaïque... En redevenant comme les autres après la fin d'une parenthèse de trois siècles pendant laquelle les Européens se sont crus radicalement différents des «autres», nous retrouvons notre humanité».

Latour (Philosophie contre sociologie des sciences. Une querelle enfin dépassée, in «Le Débat», $N^{\circ} 42$, novembre-décembre 1996, pp. 153-163) est convaincu que le divorce entre la philosophie des sciences et la sociologie, le divorce entre l'épistémologie et l'histoire sociale des sciences, ont été provoqués par une conception réduisant le social aux seuls humains, à leurs seuls rapports sociaux, à leurs seuls intérêts économiques. D’après lui «Le social se compose, en large partie, de non-humains mobilisés en son sein... Il produit ... de nouvelles associations, des combinaisons inouïes d'humains et non-humains ... une société ne se compose pas de sujet de droit en retrait par rapport aux choses, mais de multiples associations et de multiples échanges entre humains et non-humains ».

Que sont ces non-humains? D'où viennent-ils? De quels droits sont-ils titulaires? Ont-ils les mêmes responsabilités que les humains?

Je l'ai déjà dit: Latour donne aux objets un statut d'acteur et propose de remplacer l'intersubjectivité par l'interobjectivité, de définir l'interaction sociale en y introduisant les objets (Une sociologie sans objet? Remarques sur l' interobjectivité, in «Sociologie du travail», 1994, n. 4, pp. 587-607; Objet d'art, objet de science. Note sur les limites de l'anti-fétichisme, in «Sociologie de l'art», 1993, n. 6, pp. 7-24). Cependant, il ne nous dit pas comment des objets, construits ou valorisés par des acteurs sociaux, deviennent subitement, eux aussi, des acteurs protagonistes d'interactions sociales. Il y a là un mystère sociologique, voir un tour de passe-passe philosophique. A mon avis, Latour nous propose, en philosophe, des thèses sans aucun doute respectables, mais peut-on les fonder en réduisant la science à une simple croyance sociale, semblable ou analogue à la magie ou à l'idéologie d'une «illusion », la modernité? Peut-on traiter la science en tant que totalité, globalité, alors qu'elle sépare, distingue, morcelle en lots, et procède par approximations successives, par tâtonnements, corrections et rectifications? Comment oublier le fait que les savants s'intéressent avant tout à la validité des conclusions particulières sur le réel, alors que les philosophes s'intéressent à la nature de la méthode scientifique en sa globalité, que les premiers ne prêtent aucune attention à la question des fondements alors que pour les seconds elle est primordiale et absolue?

Les connaissances scientifiques passent aussi par des processus intellectuels, par des élaborations conceptuelles, par la critique du savoir scientifique. Est-il 
raisonnable de négliger ce travail essentiel et de valoriser exclusivement les conflits de pouvoir, les contraintes institutionnelles, les enjeux médiatiques, les conditionnements socio-politiques?

J'ai essayé de montrer tout au long de ces séminaires combien les choses sont complexes: les discours sur les fondements nous portent à quêter l'absolu, alors que nous devons nous contenter de réponses vérifiables bien que provisoires. C'est pourquoi je pense qu'il n'est pas opportun, ni convenable, de traiter de la science comme d'une croyance; il faut tenir compte des spécificités de l'activité scientifique, examiner ses rapports avec le contexte global et établir les dépendances et les indépendances. Traiter la science et la technique comme si elles étaient des croyances constitutives de l'idéologie de la modernité nous conduit à une impasse intellectuelle. La science et la technique sont vides de finalités, elles ne sont que des moyens au service des sociétés et celles-ci les utilisent pour le meilleur comme pour le pire. Attribuer à la science et à la technique les méfaits du colonialisme, les abus du capitalisme ou du socialisme, les totalitarismes de droite et de gauche, le développement incontrôlé, la pauvreté, le paupérisme, le chômage, l'effet de serre, la mise en danger de l'écologie de notre planète, les difficultés existentielles auxquelles nous sommes tous actuellement confrontés, la perte des liens sociaux, l'exclusion, la marginalisation et tant d'autres maux dont nos sociétés souffrent, c'est une vision simpliste et dangereuse. Simpliste parce qu'elle ne propose qu'une seule façon de voir et de concevoir un ensemble de choses complexes, celle qu'adoptent les doctrinaires du «New Age» et du «Postmodernisme »; dangereuse parce qu'elle nous replonge dans le mysticisme et dans l'irrationalisme, elle nous livre aux bizarreries des prophètes et à des pratiques intellectuelles et sociales régressives et irresponsables. Par contre, si nous voulons déchiffrer les énigmes de la vie sociale et décoder les mystères du monde naturel, si nous voulons maîtriser les événements et en contrôler les développements, le seul moyen dont nous disposons est la méthode scientifique. Grâce à sa méthode, à l'aide de données empiriques susceptibles de vérifier nos hypothèses et nos formulations théoriques, la science peut nous faire comprendre le monde dans lequel nous nous trouvons et agissons.

Nous savons depuis David Hume que l'induction nous enferme dans un cercle vicieux (soit à justifier une généralisation au moyen d'une autre généralisation légitimée par son succès passé) et qu'il est impossible de fonder logiquement la science. Karl Popper est allé plus loin dans cette même direction grâce à son principe de falsification, dont l'observation sociologique du travail dans les laboratoires a toutefois montré les limites. En effet, le travail réel des savants consiste surtout à comprendre pourquoi les hypothèses adoptées ne fonctionnent pas, plutôt qu'à en chercher la réfutation ou à en démontrer la fausseté. En outre, dans les relations causales du monde réel, il n'est pas donné d'observer un rapport d'une seule cause à un seul effet. Les phénomènes réels sont «causés » par de très nombreuses variables, ils se trouvent en situation de mutuelle dépendance.

Thomas S. Kuhn a essayé d'éliminer ces apories de la théorie poppérienne avec les concepts de «paradigme», de «science normale», de «révolution scientifique»; Imre Lakatos, pour sa part, a montré les avantages comparatifs des approches de Popper et de Kuhn; tandis que Paul K. Feyerabend a présenté les bénéfices que l'on peut tirer du pluralisme, voire de l'anarchisme méthodologique, comme vous avez pu l'apprendre en lisant les ouvrages d'Alan Chalmers. 
Pourquoi ces références à Popper, Kuhn, Lakatos et Feyerabend? Pour vous dire d'une autre manière que l'histoire des pratiques scientifiques nous prouve que la science ne constitue pas un corpus fermé de théories. Elle n'est qu'un ensemble d'instructions, de préceptes et de prescriptions servant à faire des classifications, des inférences causales, à formuler des hypothèses à vérifier, à organiser les observations en séquences logiques et cohérentes, à identifier les différents niveaux d'explications, les disparités technologiques et les diverses capacités cognitives. Les résultats produits sont universaux pour tous ceux qui appliquent, avec un esprit critique, ces modèles normatifs. Ces universaux ne sont pas éternels, jamais définitifs. Ils se modifient en fonction des contraintes culturelles, des contextes sociaux et cognitifs, de l'urgence des problèmes à résoudre, des curiosités intellectuelles à satisfaire, mais toujours selon des processus propres à l'activité scientifique, à son autonomie conceptuelle et à son organisation, à la constance et à la permanence des contrôles critiques.

Je vous ai dit que jusqu'à la fin des années '60, le paradigme sociologique dominant était celui mis au point par Robert K. Merton. Pour ce sociologue américain, la science est un processus rationnel autorégulateur, fondamentalement producteur d'effets bénéfiques et de résultats positifs, et dont les implications sociales peuvent être étudiées par la sociologie de la connaissance scientifique, et plus généralement par la sociologie de la science. Dès les années '70, face à cette conception libérale, ou modérée, l'Ecole d'Edimbourg a opposé le «programme fort», sa conception de la science comme miroir de la société, comme la résultante de déterminations et conditionnements sociaux et existentiels.

La sociologie de Merton me paraît trop optimiste, elle accorde une place excessive à la philosophie et à l'histoire au détriment de l'analyse sociologique. Par contre, les doctrines de Barnes, de Bloor, de Mulkay, de Woolgar, mais aussi de Mary Hesse (Revolution and Reconstruction in the Philosophy of Science, Brighton, Harvester, 1980) et de Steven Shapin (History of science and its sociological reconstructions, in «History of Science», 20, 1982, pp. 157-211) ne donnent aucune importance au processus temporel et dynamique de l'histoire. Pour ces chercheurs, l'histoire est statique et contextuelle. Les discussions scientifiques sont des négociations, les écrits des savants des moyens qui permettent d'obtenir le consensus des collègues et de se procurer ainsi des gratifications et des récompenses honorifiques ou pécuniaires. Les sciences n'auraient aucune spécificité constitutive. Elles ne seraient pas porteuses de critères de validité, ni de vérité.

Michael Lynch a montré (Technical Works and critical Enquiry: Investigations in a scientific Laboratory, in «Social Studies in Science», 12, 1982, pp. 499533) la fragilité de ces thèses et rapporté les jacasseries de leurs auteurs. Et j'ajoute: leur incapacité à admettre qu'il faut distinguer les savants et la science, la science en tant que méthode et la science en tant que corpus; leur obstination à nier l'existence de nombreux niveaux d'explications pas nécessairement incompatibles, ou le fait que la cause doit être séparée de la conséquence.

En conclusion, si Merton fait une part trop petite à l'analyse sociologique, l'Ecole d'Edinburgh et celle qui est en train de se former à Paris autour de Latour et Callon lui en font une trop grande. Pour ma part, je répète que le sociologue de la connaissance scientifique ne doit pas abandonner la tradition mertonienne, mais qu'il ne doit pas non plus négliger certaines suggestions critiques du «programme 
fort», et qu'il devrait aussi faire davantage appel à l'histoire. La science est le produit d'un processus dynamique dans le temps et dans l'espace. La prise en compte de la temporalité peut nous expliquer à la fois son individualité et son universalité, sa puissance et sa faiblesse, son épanouissement et son déclin.

Deux exemples suffiront à illustrer ces propos. Les découvertes génétiques de Sergej Cetverikov, en URSS, dans les années '20, sont effacées des programmes de recherche par Trofim Denisovic Lysenko, cependant elles réapparaissent ailleurs, dans les années ' 30 et '40, grâce aux généticiens occidentaux R.A. Fischer, J.B.S. Haldane et Sewall Wright. La science arabe du XIII et du $\mathrm{XIV}^{\mathrm{e}}$ siècles est à l'origine de découvertes parmi les plus fondamentales. Elle a trouvé, de façon magistrale et originale, des solutions perspicaces à des problèmes d'une complexité extraordinaire. Dans son livre The Rise of Modern Science (Cambridge, University Press, 1993), Toby Huff montre que lorsque les écoles théologiques imposèrent l'idée que tout était dit dans le «Coran», qu'il n'y avait rien à dévoiler et à découvrir en dehors des révélations faites par Allah à Mahomet, que la prétention à éclaircir les mystères de l'Univers était une insulte à l'omniscience de Dieu et que le travail critique était un blasphème s'il ne s'arrêtait pas devant la vérité de la révélation contenue dans le texte sacré, la chimie, l'astronomie, la physique furent aussitôt balayées par le fondamentalisme religieux. L'appauvrissement culturel des pays arabes fut considérable, mais la science continua son chemin ailleurs. Le génie arabe lui avait fait défaut, mais elle a poursuivi son travail et sa quête.

En conclusion, pour bien structurer son savoir, pour élaborer des connaissances véritablement solides, la sociologie de la connaissance scientifique doit être une sociologie historique des théories et des pratiques sociales des sciences et des techniques qui sont à la base de la connaissance scientifique.

Les travaux sociologiques de Raymond Boudon, mais également ceux du physicien Jean-Marc Lévy-Leblond, pour qui la science est une somme de savoir mais aussi une culture (La pierre de touche. La science à l'épreuve, Paris, Gallimard, 1996, et Aux contraires. L'exercice de la pensée et la pratique de la science, Paris, Gallimard, 1996), nous prouvent que cette tâche est réalisable. Il me plaît d'achever ces leçons par une citation tirée de Aux contraires... La voici: «Le temps est venu d'une réflexion sur les rapports entre théories scientifiques et pensée commune... Devant la complexité du monde, les sciences ont pour stratégie d'isoler progressivement certains secteurs, d'y circonscrire des phénomènes particuliers et de les spécifier toujours plus finement, jusqu'à, idéalement, en maîtriser toutes les conditions ... comment croire que le progrès intellectuel puisse suivre un chemin inverse ?... (p. 13). Pour l'instant, c'est d'emblée une attitude critique qui est de mise. Une telle posture permet de prendre avantage des limitations mêmes du savoir scientifique. Si les connaissances que nous donnent les sciences sont beaucoup plus spécifiques et particulières pour servir d'exemples ou de modèles à la pensée en général, elles sont assez précises et articulées pour lui offrir des contre-exemples et des anti-modèles. Non point simple arsenal d'outils intellectuels, mais banc d'essais pour tester leur validité. On s'aperçoit alors que bien peu des instruments de pensée généraux, avec lesquels paresseusement nous tentons de comprendre le monde, résistent à leur mise à l'épreuve là où deviennent contraignantes les exigences conjointes de la rigueur et de la fécondité » (pp. 14-15). 
Certes, au cours de ces dernières années ces questions sont devenues cruciales, peut-être même primordiales. Le rôle et les fonctions des sciences et des techniques dans notre société continuent à susciter, comme toujours, les habituelles discussions anthropologiques, philosophiques et sociologiques, mais aussi des controverses politiques virulentes, des querelles culturelles irréconciliables. Très répandus sont également les sentiments d'une angoisse infinie et indéterminée face à l'expansion incontrôlée des savoirs, des savoir-faire et de tous ces nouveaux outils technoscientifiques qui transforment notre vision du monde et le monde lui-même.

Alors que la science et la technique font des progrès inouïs, alors qu'elles nous comblent encore d'étonnement, les inquiétudes et les obsessions sur la perdition de l'homme, sur la destruction de la nature, sur les projets de maîtrise de la terre et des espaces interplanétaires, sur les emprises et les aliénations de la technoscience, se multiplient et s'aggravent, lestement et implacablement. L'intégration de la science et de la technique dans les activités essentielles des Etats, la connaissance devenue élément fondamental de la force et de l'hégémonie, sous l'angle non seulement de la puissance militaire, agro-alimentaire et industrielle, mais aussi de l'économie générale tout court, provoquent des interrogations effrayées sur les dangers auxquels la vie sur notre planète est désormais exposée.

La croyance suivant laquelle la science et la technique ne voient dans le monde naturel qu'un objet de conquête, un réservoir de ressources à exploiter, l'idée qu'elles alimentent la prétention des hommes à dominer et planifier la planète, à l'exploiter afin de disposer d'énergies toujours plus puissantes, mettant ainsi en danger l'équilibre de tout l'écosystème, cette croyance, très diffuse, n'est plus l'apanage exclusif de l'homme de la rue, comme l'attestent assez régulièrement les enquêtes d'opinion de cette dernière décennie et des publications respectables.

Par exemple, la lecture des actes du colloque organisé par l'Unesco à Tokyo sur le thème «Science et culture: un chemin commun vers l'avenir», publiés en français par les Editions Albin Michel dans le volume La mutation du futur nous révèle que cette croyance attire et intrigue également les notabilités de presque toutes les communautés scientifiques au sein desquelles deux points de vue extrêmes, celui de la science et de la technique salvatrices et celui de la science et de la technique damnables, se disputent sans merci, amplifiés et simplifiés par les médias. L'affrontement de ces deux points de vue perturbe les débats scientifiques et sape la confiance des honnêtes gens dans la science et dans la technique en tant que moyens d'émancipation de l'homme, en tant que moyen pour arriver à dévoiler les mystères de la vie.

En Suisse, il a fallu voter à propos d'une initiative populaire dite «Pour la protection génétique», déposée le 25 octobre 1993, visant à inscrire dans la Constitution fédérale un article, le 24 decies, et proscrivant les recherches et les thérapies géniques, la possibilité de breveter des organismes génétiquement modifiés et interdisant leur dissémination dans l'environnement. En automne 1996, sept associations de défense de l'environnement déposèrent, à Berne, une pétition par laquelle 147.000 citoyens demandaient aux Autorités fédérales de «mettre un verrou définitif au non-sens transgénique», d' «empêcher la dissémination illégale et l'utilisation incontrôlable de soja transgénique », lequel, on le sait, résiste pourtant aux herbicides totaux.

Un sondage (1000 personnes interrogées par IHA-GfM de Nidwald), publié en novembre 1996, attestait que seulement $57 \%$ des sondés avaient une connaissance 
précise des enjeux de cette initiative populaire. Parmi les personnes interrogées et ayant pris connaissance de l'initiative, 38\% déclaraient vouloir la rejeter, 32\% l'approuvaient tandis que $30 \%$ étaient indécis. Le rejet étaient plus fort chez les femmes et plus généralement chez les électeurs de plus de cinquante ans. A peine $0,4 \%$ des personnes sondées faisaient confiance aux informations fournies par les industries du secteur des technologies génétiques, $1,5 \%$ considère fiables les autorités politiques et $29 \%$ crédibles les scientifiques. Ajoutons que l'initiative fût rejetée par le «Souverain» et le danger de la contrainte constitutionnelle ainsi écarté, au moins pour quelques années. Il n'en reste pas moins que même dans la démocratie dite de concordance, accoutumée à élaborer les consensus moyennant de longues négociations, dans cette démocratie où l'on abhorre plus que tout les barouds et les excès, les points de vue des partisans et des adversaires ont pris, sur ces questions, des allures et des tons proches du paroxysme et de l'affrontement inflexible. Pour eux la science constitue un danger. En effet, pour les uns, la recherche génétique améliorera assurément la qualité de la vie sur les plans de la santé, de la nutrition, de la longévité, de la production de végétaux et d'animaux dits transgéniques, tandis que pour les autres les manipulations des gènes provoqueront inéluctablement l'apparition de micro-organismes très virulents, d'êtres monstrueux, d'animaux et d'insectes particulièrement agressifs, et transformeront à la fois la nature et l'homme.

Les partisans de cette dernière thèse prétendent que la légitimation constitutionnelle et la reconnaissance sociale de la recherche génétique auraient des conséquences catastrophiques pour la vie sur la Terre, changeraient les relations sociales, bouleverseraient nos façons d'être, et par conséquent notre propre destinée morale jusqu'ici singulière et irremplaçable.

Certes, les historiens des idées nous apprennent que déjà Martin Heidegger, Albert Schweitzer, Bertrand Russel, Karl Jaspers et tant d'autres argumentèrent et firent valoir, au lendemain des premières explosions atomiques, des thèses assez semblables. Cependant, celles prônées aujourd'hui soit par les apologistes soit par les contempteurs sont nettement plus drastiques et plus irréversibles.

Une très grande majorité de nos contemporains estime que la science et la technique ne sont plus les moyens indispensables pour l'émancipation de l'homme, pour son affranchissement d'une nature hostile, pour refuser le monde comme donnée brute. Les savoirs scientifiques ne sont plus la résultante de choix conçus et voulus librement, et que le cas échéant nous pourrions remettre en question, mais plutôt les foucades d'un destin aveugle, imprévisible, irrépressible, piégeant nos civilisations, notre sort commun, accélérant notre déchéance d'êtres naturels et sociaux, de personnes morales.

Ainsi la science et la technique ont perdu la position de surplomb absolu, elles se trouvent désormais foncièrement impliquées dans l'existentiel et le social, transformées en partenaires de l'économie, de la politique, et plus généralement de la croissance industrielle et du développement socio-économique.

Il s'agit-là d'un changement radical. Si l'influence de la science et de la technique sur les destinées de l'humanité, sur notre destin matériel naturellement, mais aussi sur notre comportement général, intellectuel, social et spirituel, n'est plus positive, alors il faudra remanier les fondements même de la science, repenser les notions de liberté de recherche, de recherche fondamentale, libre ou orientée, de recherche appliquée qui vise à trouver la solution d'un problème 
pratique, d'études appliquées qui se concentrent sur l'exploitation effective des connaissances dans l'ordre de la production des biens et des services. Il faudra alors opérer une véritable révolution épistémologique, pour ne pas dire culturelle, pour discerner la rationalité scientifique des divers autres modes de validation de l'idéologie, de la politique, du droit, de l'économie et de la communication médiatique.

De quelle façon et selon quelles modalités pourrions-nous reconstruire les fondements d'une nouvelle pratique scientifique alors même que la boîte noire contenant les systèmes cognitifs de la science et de la technologie en tant qu'institutions sociales est toujours complètement encodée et cela malgré les efforts surdimensionnés pour la décoder du «programme fort», du «programme dur» et les tentatives disparates de l'anthropo-sociologie de la technoscience? Le cas échéant, quelles significations et fonctions pourrait avoir cette nouvelle pratique scientifique dans notre société à l'aube du troisième millénaire? Doit-on considérer comme surannées les distinctions entre la recherche de la vérité et la délibération en vue de l'action, entre la société de pensée et la société de vie, entre les délibérations pratiques et la connaissance pour la connaissance? Y a-t-il d'autres chemins à arpenter entre l'artificialisme généralisé, postulant que tout est construit, et le retour à l'empirisme qui récuse la fermeture du sujet sur ses constructions artificielles et réaffirme l'existence d'un monde englobant et dépassant la pensée? Au-delà ou en deçà du constructivisme et du naturalisme, y auraitil d'autres approches plus attentives à la complexité des interactions entre le savoir et la réalité, entre le sujet et l'objet, entre la finitude du monde physique et social, sa dégradation irréversible, et l'équilibration majorante des nouveaux possibles de Jean Piaget?

Il est difficile de trouver des réponses plausibles à toutes ces questions.

En tout cas, pour bien structurer le savoir, pour produire et fabriquer des connaissances véritablement solides, il ne faut pas larguer les acquis du passé.

Certes, ce travail aux résultats aléatoires est difficile et complexe, mais pouvons-nous négliger l'invite que nous adressa Karl Jaspers, au lendemain de la dernière guerre mondiale, dans son ouvrage sur l'avenir des hommes et des cultures de l'ère atomique?

Pour mémoire, voici cette invite: «Que l'utopique soit possible, c'est une confiance en nous qui nous l'assure, confiance qui n'est pas fondée en ce monde, mais qui n'est donnée pourtant qu'à celui qui fait ici ce qu'il peut.»

Dans les temps à venir il faudra analyser en profondeur ces problématiques, étudier de plus près si les sciences et les techniques sont vraiment et doivent rester des univers autoréférentiels, susceptibles de déterminer à eux seuls les changements dans la société et de la société. Il faudra également formuler des meilleurs questions sur les limites qu'il faudrait peut-être fixer à la liberté de recherche, sur l'opportunité d'une surveillance que la société pourrait ou devrait exercer sur les chercheurs pour prévenir et réprimer les abus, sur nos devoirs et responsabilités, en tant que citoyens, chercheurs et enseignants, face aux utilisations des savoirs scientifiques et à l'utilisation du savoir-faire technique, à la finitude du monde naturel et aux bouleversements de toute sorte provoqués par les processus de mondialisation et de globalisation des cultures et de l'économie. Enfin, il faudra mieux comprendre si le progrès techno-scientifique peut, à lui seul, entraîner le progrès moral ou bien si le social est indispensable, si des transformations radi- 
cales de la société sont indispensables pour orienter et valoriser la science et la technique en progrès moral.

Avec d'autres mots: il faudra travailler davantage, avec des philosophes et des historiens, pour mieux fonder la sociologie de la connaissance scientifique.

Genève 